\title{
Sicherheit statt Freiheit?
}

INAUGURAL-DISSERTATION zur Erlangung des Doktorgrades der Geschichts- und Gesellschaftswissenschaftlichen Fakultät der Katholischen Universität EICHSTÄTT-INGOLSTADT

vorgelegt von JENS CHRIstian HORNig 85072 Eichstätt Rosenweg 13 2009 


\section{Vorwort}

Eine Doktorarbeit ist ohne Hilfe und Unterstützung nur schwer möglich. Eine Vielzahl von Faktoren hat Einfluss auf die wissenschaftliche Leistung, die im Rahmen einer Promotion erbracht wird. Dazu zählen fachliche Aspekte ebenso wie zwischenmenschliche Formen der Unterstützung, die oftmals bei der Erstellung der Arbeit den wichtigsten Anteil am Erfolg haben.

Für all die genannten Dinge gilt mein Dank meinem Doktorvater Prof. Dr. SIEGFrIED LAMNEK von der Katholischen Universität Eichstätt, der diese Doktorarbeit kontinuierlich von der Themenfindung bis zur Vollendung intensiv betreut hat und mir mit seiner umfassenden Unterstützung zu jeder Zeit zur Seite stand.

Im Rahmen dieser Dissertation wurde eine Reihe von Interviews mit verschiedenen Personen geführt, die alle mit dem Gegenstand in Verbindung stehen. Besonders bedanken möchte ich mich an dieser Stelle bei Frau SABINE LeUtheusser-SCHNARREnBERger, die sich mir freundlicherweise für eine ausführliche Expertenbefragung zur Verfügung gestellt hat. Der dadurch gewonnene Einblick in die politische Praxis - dies bezieht sich sowohl auf Frau Leutheusser-Schnarrenbergers Erfahrungen als Bundesministerin der Justiz als auch auf ihre derzeitige Tätigkeit als Abgeordnete des Deutschen Bundestages und als Vorsitzende der bayerischen FDP - hat meine Perspektive erweitert und meinen Blick für bestimmte Aspekte meines Themenfeldes geschärft.

Ich möchte hier abschließend noch meiner Hoffnung Ausdruck verleihen, dass für alle meine Gesprächspartner in den letzten Jahren das Thema dieser Doktorarbeit ebenso fesselnd und interessant wie für mich war und somit auch für sie die investierte Zeit und Mühe keine verlorene war. 
Meine Freundin Sylvia Henne war mir während der gesamten Zeit Inspiration und wichtigste Motivation und stand immer fest an meiner Seite. Vielen Dank für das entgegen gebrachte Vertrauen.

Meinen Eltern Christel und Gunter Hornig gilt abschließend der größte Dank. Sie sind meine Vorbilder und haben mich fortwährend unterstützt und ermutigt und damit die Dissertation letztendlich erst in dieser Form möglich gemacht. 
1. Einleitende Gedanken 1

1.1 Zentrale Fragestellungen 2

1.2 Aufbau und inhaltliche Schwerpunkte 6

2. Grundlagen und Konzeptionen von Freiheit und Sicherheit 9

$\begin{array}{lll}2.1 & \text { Freiheit } & 10\end{array}$

2.1.1 Eine Typologie von »Freiheit» 12

2.1.2 Handlungs-Determinismen und die Willensfreiheit 20

2.1.3 Eine soziologische Sichtweise - Freiheit innerhalb eines

2.1.3.1 Interdependente menschliche Gesellschaft 32

2.1.3.2 Freiheit innerhalb interdependenter Gesellschaften:
Kontrolle als entscheidender Faktor

2.1.3.3 Wissen und andere sekundäre Bedingungen für Freiheit $\quad 41$

$2.2 \quad$ Sicherheit 51

2.2.1 Sicherheit als Begriff $\quad 52$

$\begin{array}{lll}\text { 2.2.2 Die Produzenten von Sicherheit } & 61\end{array}$

2.2.3 Sicherheit und Kontrolle 66

2.2.4 Sicherheit und Risiko als menschliche Grundbedürfnisse? 77

2.2.5 Wissen - Objektive und subjektive Sicherheit 80

2.3 Das Verhältnis von Freiheit und Sicherheit 86

2.3.1 Das Zusammenwirken von Freiheit und Sicherheit $\begin{array}{ll}\text { Ideengeschichte } & 87\end{array}$

2.3.2 Privatheit als Schnittstelle von Freiheit und Sicherheit 98

2.3.3 Zusammenfassung und zentrale Fragestellungen 103 
3. Chronologie des Wandels von Sicherheit und Freiheit

106

3.1 Die Ausgangslage: Der freiheitliche Verfassungsstaat

$\begin{array}{ll}\text { Bundesrepublik Deutschland } & 107\end{array}$

$\begin{array}{ll}\text { 3.1.1 Der Verfassungsstaat } & 107\end{array}$

$\begin{array}{ll}\text { 3.1.2 Freiheit im Grundgesetz } & 109\end{array}$

$\begin{array}{lll}\text { 3.1.3 Sicherheit im Grundgesetz } & 117\end{array}$

$\begin{array}{ll}\text { 3.1.4 Wichtige politische Akteure und Abläufe } & 121\end{array}$

$\begin{array}{lll}3.2 & \text { Die Notstandsgesetze } & 131\end{array}$

$\begin{array}{lll}\text { 3.2.1 Die Ausgangslage } & 131\end{array}$

3.2.2 Die einzelnen Veränderungen durch die Notstandsgesetze 134

3.2.3 Die Folgen für Sicherheit und Freiheit 136

3.3 Terrorismus I - die Rote Armee Fraktion (RAF) 140

$\begin{array}{ll}3.3 .1 & 141\end{array}$

$\begin{array}{lll}\text { 3.3.2 Anti-Terror-Maßnahmen } & 143\end{array}$

3.3.3 Bewertung: Freiheit und Sicherheit vor dem Hintergrund der RAF 147

3.4 Das Volkszählungsurteil des BVerfG - Das Recht auf informationelle Selbstbestimmung $\quad 150$

3.4.1 Vorgeschichte des Urteils 150

3.4.2 Zentrale Bestimmungen des Urteils 154

3.4.3 Konsequenzen und Wirkungen des Urteils 156

$\begin{array}{lll}3.5 & \text { Europäische Vereinigungsprozesse } & 160\end{array}$

$\begin{array}{lll}3.5 .1 & \text { Deutsche Vereinigung } & 160\end{array}$

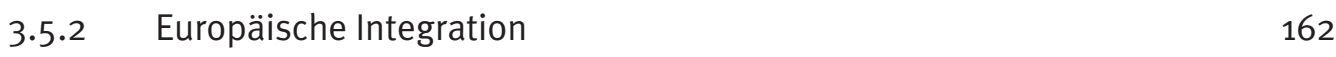

$\begin{array}{ll}3.5 .3 & \text { Bewertung } \\ & 165\end{array}$

$\begin{array}{lll}3.6 & \text { Der große Lauschangriff } & 169\end{array}$ 
$\begin{array}{lll}\text { 3.6.1 Die wichtigsten Bestimmungen } & 169\end{array}$

$\begin{array}{lll}\text { 3.6.2 Folgen der Grundgesetzänderung } & 172\end{array}$

$\begin{array}{lll}3.6 .3 & \text { Bewertung } & 174\end{array}$

3.7 Terrorismus II - 9/11 176

$\begin{array}{lll}\text { 3.7.1 Eine neue Form des Terrorismus } & 176\end{array}$

3.7.2 Neue Sicherheitsbedürfnisse nach den Anschlägen 177

$\begin{array}{lll}3.7 .3 & \text { Bewertung } & 180\end{array}$

$3.8 \quad$ BKA-Gesetz von $2008 \quad 182$

$\begin{array}{lll}3.9 & \text { Zusammenfassung } & 187\end{array}$

4. Aktuelle Entwicklungen - Das Verhältnis von

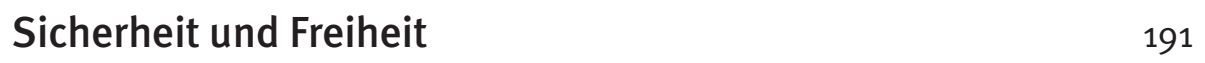

4.1 Der Wandel von Privatheit 191

4.1.1 Schnittstellen und Kernbereiche 192

4.1.2 Eingriffe in die Privatheit - Kontrollverluste 194

4.2 Akteure in der Überwachungsgesellschaft 199

4.2.1 Behörden und staatliche Organe 202

4.2.2 Gruppierungen 206

4.2.3 Individuen - die Veröffentlichung des Privaten 208

4.2.4 Exkurs - Gated Communitys 212

4.3 Sicherheit in der Präventionsgesellschaft 213

4.3.1 Die Funktionsweise des Präventionsstaates 215

4.3.2 Sachzwänge als wichtigste Bedingung 221

4.4 Technik und Daten 223

$\begin{array}{lll}\text { 4.4.1 Technische Entwicklungen } & 223\end{array}$ 
4.4.2 Datenbanken und Kontrolle 228

4.4.3 Freiheit zurückgewinnen 232

4.5 Zusammenfassung und Bewertung 237

4.5.1 Freiheit durch Gegenmaßnahmen? 237

4.5.2 Sicherheit durch Fremdkontrolle? 242

4.5.3 Sicherheitsversprechen - statt Freiheit 244

5. Schlussbetrachtung 251

$\begin{array}{ll}\text { Literaturverzeichnis } & 258\end{array}$

$\begin{array}{ll}\text { Webseiten ohne Autorenangabe } & 258\end{array}$

$\begin{array}{ll}\text { Quellen mit Autorenangabe } & 260\end{array}$ 


\section{Einleitende Gedanken}

Im Juni 2007 trafen sich die Staats- und Regierungschefs der G 8-Staaten in Heiligendamm in Mecklenburg-Vorpommern zu ihrem turnusmäßigen Gipfeltreffen, um aktuelle politische Problemstellungen zu besprechen. Die Sicherheitsmaßnahmen, die zum Schutz der Gipfelteilnehmer getroffen wurden, waren umfangreich und vielfältig. Das Kernstück des Sicherheitskonzeptes bestand aus einem 13,5 Kilometer langen und 2,50 Meter hohen Sicherheitszaun aus Stahlbeton, Stahlgittern und Stacheldraht, der nur durch zwei Sicherheitsschleusen passierbar war und zusätzlich durch Bewegungsmelder und Kameras verstärkt wurde (vgl. sueddeutsche.de 2007 a). Die dadurch von der Außenwelt abgeschnittenen Bewohner von Heiligendamm und verschiedene Dienstleistungsunternehmen, die rund um den Gipfel tätig waren, wurden registriert und fotografiert (vgl. ndr.de 2007). Insgesamt 16000 Einsatzkräfte der Polizei, der Bundeswehr und des BKA sollten dafür sorgen, dass die 100 Kilometer weit reichende Flugverbotszone, das 11 Kilometer umfassende Sperrgebiet zu Wasser und die Zugangsbeschränkung des Tagungsortes eingehalten wurden (vgl. BOECKER 2007). Ergänzt wurden die örtlichen Restriktionen durch ein weiträumiges Demonstrationsverbot, das nach einer zunächst erfolgreichen Klage vor dem Verwaltungsgericht Schwerin durch das Oberverwaltungsgericht Mecklenburg-Vorpommern weitgehend bestätigt wurde (OVG Mecklenburg-Vorpommern 3 M 53/07 1 B 243/o7 VG SN).

Im Vorfeld des Gipfeltreffens wurden ca. 40 Wohnungen von potenziellen G-8-Gegnern durchsucht und gegen mehr als "20 mutmaßliche Terroristen « (sueddeutsche.de 2007 b) ein Ermittlungsverfahren wegen Bildung einer terroristischen Vereinigung nach §129a StGB eingeleitet. Zusätzlich wurden bei 
verschiedenen vorab Verdächtigten Körpergeruchsproben (vgl. sueddeutsche.de 2007 c) genommen, um diese später mit Hilfe von Polizeihunden identifizieren zu können. Da dem G-8-Gipfel mehrere Farb- und Brandanschläge in Hamburg vorausgingen, wurden richterlich genehmigte Briefkontrollen durchgeführt, um Bekennerschreiben bestimmten Briefkästen zuordnen zu können und dann eine Observierung der Verdächtigen zu ermöglichen (vgl. sueddeutsche.de 2007 e). Das Gesamtkonzept am Tagungsort selbst ging auf, die Teilnehmer des Gipfeltreffens konnten sich relativ ungestört den politischen Fragen und Inhalten widmen, die auf der Tagesordnung standen. Während des Gipfeltreffens kam es aber im unweit entfernten Rostock zu Krawallen und Zusammenstößen zwischen Demonstranten und der Polizei. Die von verschiedenen Organisationen initiierte friedliche Demonstration, die unter dem Motto »eine andere Welt ist möglich« (ebd.) stand, entwickelte sich auf diese Weise zu einer Straßenschlacht. Rund 430 Polizisten (vgl. spiegel.de 2007) wurden verletzt, zahlreiche Randalierer wurden festgenommen und anschließend in "Gitterkäfigen« (PRANTL 2007a) festgesetzt. Im Rahmen des behördlichen Vorgehens gegenüber den Demonstranten leistete auch die Bundeswehr einen unmittelbaren Beitrag, als am 5. Juni 2007 Tornados der Bundeswehr in rund 150 Metern Flughöhe ein Camp der Demonstranten nahe der Ortschaft Reddelich überflogen und mit hochauflösenden Kameras Fotografien der Anlage machten (vgl. sueddeutsche.de 2007 d).

\subsection{Zentrale Fragestellungen}

Die Ereignisse von Heiligendamm sind für diese Arbeit in verschiedener Hinsicht bedeutsam. Sie illustrieren hervorragend, wie an bestimmten wichti- 
gen Ereignissen die Einflüsse, die Sicherheit und Freiheit betreffen, sichtbar werden. Das Augenmerk gilt aber nicht nur markanten Einzelereignissen, sondern auch Abläufen, die oft nicht ins Auge stechen und die ihre Wirkung weniger auffällig entfalten. Die besonderen Intentionen der Beteiligten sind dabei wichtig, da die Vorstellungen von Sicherheit und Freiheit oftmals weit auseinander liegen. In der Tat zeichnet sich der Begriff Freiheit durch eine besondere Vielfalt an Bedeutungszuschreibungen aus. Nicht in jedem Fall ist klar zu erkennen, welche Inhalte exakt mit dem Begriff der Freiheit verbunden werden. Es gibt inn in der politischen Praxis, etwa bei der Zuordnung zu bestimmten politischen Inhalten oder auch im Namen von Parteien. Er existiert im Bereich des Rechts, etwa in Form von Freiheitsrechten wie der Meinungsfreiheit (Art. 5 GG), der Versammlungsfreiheit (Art. 8 GG) oder dem Recht, seinen Arbeitsplatzo frei zu wählen (Art. 12 GG). Da sich mit dem Begriff Freiheit nicht nur die Soziologie sondern auch andere Wissenschaften wie Philosophie, Theologie und Politikwissenschaft teilweise über Jahrhunderte hinweg beschäftigt haben, existieren diverse Bedeutungszuschreibungen und Definitionen von Freiheit, aus denen für diese Arbeit ein angepasstes, soziologisch brauchbares Verständnis von Freiheit entwickelt werden soll.

Für den Begriff Sicherheit gilt Ähnliches, da auch er in einer langen historischen Tradition und mit vielen Bereichen des alltäglichen Lebens in Verbindung steht und deshalb einer präzisen Fassung bedarf, um für die soziologische Analyse brauchbar zu sein. Wie für Freiheit gilt auch für Sicherheit, dass erst die partielle oder zeitweilige Abwesenheit von beiden ihre Bedeutung hervortreten lässt. Erst in der Unfreiheit und der Unsicherheit werden die entscheidenden Merkmale und Voraussetzungen für beide deutlich sichtbar und handlungsrelevant. 
Der Titel »Sicherheit statt Freiheit?« impliziert aber auch, dass die beiden Phänomene nicht jeweils für sich betrachtet werden dürfen. Es geht in gleichem Maße um das spezielle Zusammenwirken von Sicherheit und Freiheit. Die Verknüpfung der Begriffe mit "statt« soll auf einen Zusammenhang und auch auf eine Entwicklung verweisen, wobei sich bei fortschreitender Analyse ergeben hat, dass die recht einfach und linear anmutende Formel »Sicherheit statt Freiheit« um einiges komplexer ist als die bloße Formulierung erahnen lässt. Es bedarf der Klärung, ob ein Mehr an Sicherheit generell Freiheit reduziert oder diese im Gegenteil sogar fördern kann. Ebenso gilt es herauszuarbeiten, ob der Zusammenhang ein unmittelbarer oder ein indirekter ist und ob es weitere zentrale Aspekte gibt, welche Freiheit und Sicherheit gleichermaßen entscheidend beeinflusst und verändert haben.

In jedem Fall versucht die Arbeit, der Tatsache Rechnung zu tragen, dass sie einen Prozess untersucht, dessen besondere Dynamik berücksichtigt werden muss. Wichtig sind dabei auch die unterschiedlichen Geschwindigkeiten und das Ausmaß, mit denen sich Freiheit und Sicherheit in Deutschland entwickelt haben. Die Ereignisse von Heiligendamm sind ein jüngeres Beispiel dafür, wie Sicherheit in Deutschland realisiert werden kann und auch wird. Das Vorgehen der Einsatzkräfte stieß sowohl im Vorfeld, während und auch nach der Durchführung auf Kritik, die aus unterschiedlichen Richtungen und in verschiedenen Härtegraden formuliert wurde. Unter dem Titel »Der artgerechte Staat« kommentierte Heribert PRAntl (2007a) in der Süddeutschen Zeitung die Ereignisse und stellte fest, dass »das Recht der inneren Sicherheit in Deutschland (.) gekennzeichnet vom Verlust der Maßstäbe und von der Veralltäglichung der Maßlosigkeit« (ebd.) sei. Ausgangspunkt seiner Argumentation war die Unterbringung der festgenommenen Demonstranten in 
Gitterkäfigen - „20 Menschen auf 25 Quadratmeter, Tag und Nacht beleuchtet und bei all ihren Regungen gefilmt« (ebd.) - die er mit der ab 2008 verbotenen Käfighaltung von Legehennen verglich und zu einer umfassenden Kritik an verschiedenen gesetzlichen Regelungen, welche den Bereich Sicherheit betreffen, ausbaute.

HANS-ULRICH JÖRGES kommentierte im Stern die Geschehnisse von Heiligendamm und stellte unter Rekurs auf das seiner Ansicht nach »martialische Aufrüsten« (JöRGES 2007: 72) des Staates eine kontinuierliche, 50 Jahre andauernde Entwicklung des liberalen Rechtsstaates hin zu einem »rasenden Sicherheits- und Überwachungsstaat« (ebd.) fest. JÖRGES benannte in dem Artikel die Psyche der Innenminister SCHILY und SCHÄUbLE und deren besondere Biografien als Grund für obige Entwicklung und stellt am Ende seines Artikels die Frage, ob Deutschland überhaupt noch eine »lupenreine Demokratie« (ebd.) sei.

Auch von Seiten der Politik wurde Kritik am Vorgehen der Einsatzkräfte von Heiligendamm laut. Im Innenausschuss des Deutschen Bundestages wurde die "Dimension des Einsatzes« (vgl. bundestag.de 2007a), die Einschränkung der Versammlungsrechte und vor allem der Einsatz der tief fliegenden Tornados als Einsatz der Bundeswehr parteiübergreifend kritisiert. Insgesamt hätten die Einsatzkräfte aber ihre Aufgabe gut bewältigt (ebd.).

Es soll an dieser Stelle vorerst darauf verzichtet werden, die einzelnen Sicherheitsmaßnahmen und freiheitsbegrenzenden Maßnahmen von Heiligendamm en detail zu hinterfragen. Ebenso sollen die oben aufgeführten Stellungnahmen nur illustrieren, von welcher Art und von welcher Intensität die Kritik sein kann, die vor allem an staatlichem Handeln im Bereich von Sicherheit und Freiheit geübt wird. Die von JöRGES angesprochene Entwicklung ist 
ein entscheidender Faktor für diese Arbeit. Das Verhalten politischer Entscheidungsträger wird ein weiterer wichtiger Gegenstand der Analyse. Die Analyse soll erweisen, ob sie stetig bis heute verlaufen ist und wie sich Freiheit und Sicherheit entwickelt haben. Muss man wie bei einem Nullsummenspiel davon ausgehen, dass sich das Maß an Freiheit reduziert hat und dass an ihre Stelle Sicherheit getreten ist, gewissermaßen in Form einer Verschiebung oder als eine Art Kompensation? Gibt es wirklich mehr Sicherheit statt Freiheit oder haben sich beide Aspekte unabhängig voneinander verändert und entwickelt?

\subsection{Aufbau und inhaltliche Schwerpunkte}

Bevor die oben angesprochenen Forschungsfragen im Laufe der Arbeit beantwortet werden, soll in diesem Kapitel auf die wissenschaftliche Herangehensweise eingegangen und die inhaltlichen Schwerpunkte kurz dargestellt werden. Vorab sei dazu angemerkt, dass für diese Analyse keine Daten im Sinne einer eigenständigen empirischen Untersuchung erhoben wurden, da dies für den gewählten Forschungsansatz nur bedingt als brauchbar erschien. Stattdessen wurde der Schwerpunkt auf eine Untersuchung der existierenden, vielfältigen Literatur gelegt, zusammen mit einem besonderen Augenmerk auf die rechtlichen Entwicklungen und die damit verbundenen gesetzlichen Veränderungen.

Im Mittelpunkt der Untersuchung steht die oben angesprochene Entwicklung: Freiheit und Sicherheit sind keine Konstanten, sondern haben sich seit der Gründung der Bundesrepublik Deutschland massiv verändert. Trotz und wegen dieser Dynamik wird sich diese Studie an wichtigen Zäsuren orientie- 
ren, welche ausschlaggebend für die Entwicklung bis heute waren. Besonders wichtig wird dabei sein, nachzuvollziehen, ob mehr Sicherheit unmittelbar auf Kosten von Freiheit geschaffen wurde.

Entlang der oben angesprochenen »Meilensteine« (Kapitel 3), die von besonderer Bedeutung waren, organisiert sich die Analyse, wobei auffallend ist, dass sich die wichtigen Ereignisse - auch diese Tendenz ist ein typisches Charakteristikum für die Entwicklung von Sicherheit und Freiheit - in jüngerer Vergangenheit häufen. Mit den Ereignissen von Heiligendamm, den OnlineDurchsuchungen von Festplatten und den sich ausweitenden Datenschutzproblemen endet der analytische Prozess. Alle relevanten Ereignisse, die sich später ereignet haben, werden zusammen mit verschiedenen internationalen Erscheinungen aus der eigentlichen Analyse herausgenommen und abschlieBend in einem eigenen Kapitel behandelt, das versucht, unter anderem eine Prognose für den weiteren Verlauf zu entwickeln.

Es soll an dieser Stelle ausdrücklich betont werden, dass die angesprochenen Ereignisse und Veränderungen nach bestimmten theoretischen Gesichtspunkten selektiert wurden, was Vollständigkeit als Anspruch der Studie ausschließt. Informierte Leser werden verschiedene Dinge vermissen, was aber nicht zuletzt der Vielschichtigkeit und Komplexität des Themas geschuldet ist, das Vollständigkeit ohne Einschränkung kaum möglich macht.

Für die Strukturierung der jahrzehntelang fortschreitenden Veränderungen wurden verschiedene Vorgehensweisen entwickelt, die aber im Fortgang der Arbeit immer wieder hinterfragt und verworfen wurden. Um Redundanzen zu vermeiden und um mehr Übersicht in der Darstellung zu erreichen, wurde letztlich ein chronologischer Aufbau beibehalten, ohne nach Sicherheit und Freiheit in zwei getrennten Abschnitten zu gliedern. 
Die sich anschließende Zusammenfassung und die weitere Interpretation der Ereignisse arbeiten dann die wichtigsten Entwicklungen im Einzelnen heraus und liefern ein Gesamtbild der Entwicklungen mit wichtigen Erkenntnissen, Wirkzusammenhängen und Prognosen.

Die Grundlage der oben beschriebenen Untersuchungsschritte bildet eine theoretische Diskussion der zentralen Begriffe Sicherheit und Freiheit, die auch die besonderen Wechselwirkungen beider berücksichtigt. Von besonderem Interesse ist dabei die Einordung der Entwicklungen vor dem Hintergrund von Überwachungs- und Präventionsgesellschaft und vor allem auch die Frage, wie sich die Entwicklungen von Sicherheit und Freiheit vor dem Hintergrund dieser Gesellschaftsmodelle einordnen lassen. Unter besonderer Berücksichtigung der technischen Entwicklungen soll diskutiert werden, wie und vor allem ob Freiheit in diesen Gesellschaften bewahrt oder zurückerlangt werden kann. Eine Diskussion der zentralen Erkenntnisse bildet den Abschluss der Untersuchung. 


\section{Grundlagen und Konzeptionen von Freiheit und Sicherheit}

Freiheit und Sicherheit sind Begriffe, die einer präzisen und möglichst umfassenden Darstellung bedürfen. Das ist zum einen der Tatsache geschuldet, dass jede Analyse eines Gegenstandes zu verschiedenen Zeitpunkten einer genauen inhaltlichen Fassung bedarf. Dies gilt zum anderen aber auch dann, wenn man Veränderungen und Verschiebungen von bestimmten Phänomenen dokumentieren möchte: Man kann nur schwer Aussagen über einen Gegenstand machen, wenn nicht klar ist, was exakt damit gemeint ist. Im Bereich der Freiheit - und mit bestimmten Einschränkungen auch im Bereich Sicherheit muss man sich darüber hinaus inhaltlich mit der problematischen Frage auseinander setzen, ob diese realiter auch existieren.

Ein Begriff ganz allgemein ist nämlich zunächst einmal lediglich ein bestimmter Vorstellungsinhalt, der durch ein Wort oder eine Wortkombination näher bezeichnet wird (vgl. MAYnTZ 1972: 9). Durch die »beschreibende Aufzählung des durch ein bestimmtes Wort (Definiendum) gekennzeichneten Vorstellungsinhalts (Definiens)« (ebd.: 14) werden die einzelnen Inhalte des Begriffs definiert. Zu unterscheiden sind zwei verschiedene Typen von Definitionen: Als Realdefinitionen werden dabei diejenigen bezeichnet, die bestimmte Behauptungen über die Qualität eines Phänomens aufstellen (vgl. ebd.15). Im Gegensatz dazu sagt eine Nominaldefinition aus, »mit welchem Wort wir einen Gegenstand bezeichnen wollen, der die im Definiens genannten Eigenschaften« (ebd.:16) besitzt. Auf keinen Fall sind Begriffe identisch mit den verschiedenen Phänomenen, die mit den Vorstellungsinhalten in Relation stehen (vgl. ebd.:9). Sie bilden stattdessen die Wirklichkeit lediglich ab und helfen dabei, die Erfahrungs- und Wahrnehmungswelt zu strukturieren. 
Im Bereich der Medien und in der Alltagssprache werden die Ausdrücke Freiheit und Sicherheit inflationär verwendet. Ohne dies umfassend empirisch prüfen zu können oder auch nur zu wollen, kann man feststellen, dass durch den vielfältigen Gebrauch der Begriffe, der auf verschiedenen, individuell bestimmten Assoziationen zu den Begriffen beruht, ebenfalls inhaltliche Klarheit verloren geht.

Die folgenden Abschnitte legen das »theoretische« Fundament für die sich anschließenden weiterführenden Überlegungen und versuchen, die oben skizzierten Unklarheiten zu minimieren und die beiden zentralen Begriffe Freiheit und Sicherheit so zu definieren, dass weitergehende Aussagen über ihre jeweilige Entwicklung getroffen werden können.

\subsection{Freiheit}

Freiheit ist zunächst ein Begriff, dem man sich auf höchst unterschiedliche Art und Weise thematisch nähern kann. Allen Arten der Annäherung ist gemein, dass nicht von vorneherein klar ist, welche Inhalte und Bedeutungen damit genau verbunden werden. Was meint Freiheit in den unterschiedlichen Vorstellungsinhalten und welche Konsequenzen ergeben sich daraus für das Leben der Menschen?

Die verfügbare wissenschaftliche Literatur füllt ganze Bibliotheken mit unterschiedlichen Begrifflichkeiten. Freiheit wird disziplinenübergreifend als wichtig erachtet und beschäftigt Theoretiker aller Art schon seit Jahrhunderten. Die wissenschaftliche Literatur ist dabei keineswegs einheitlich. Bei einem Teil der Veröffentlichungen herrscht bereits Unklarheit darüber, ob es Freiheit überhaupt geben kann oder ob sie stattdessen nur eine Scheinfreiheit (vgl. u.a. 
SINGER 2007) darstellt, die lediglich vorgegaukelt wird. Neben dieser Scheinfreiheit als Wahrnehmungsproblem widmen sich andere Autoren in ähnlicher Weise dem Thema, indem sie untersuchen, ob der Mensch frei ist oder ob er durch verschiedene Determinanten (vgl. u.a. PAUEN 2004) eingeschränkt und bestimmt ist. Ein Großteil der Publikationen behandelt Freiheit gewissermaßen als Axiom: Sie setzen sie mehr oder weniger unreflektiert als gegeben voraus und entfalten ihre Gedanken auf dieser Basis (vgl. u.a. RouSSEAU 1964, LASKI 1945, VEIT 1957). Wichtig ist hier der Umstand, dass manch wissenschaftliche Position, die auf Freiheit als immanente Voraussetzung gründet, ganz einfach die allägliche Freiheitswahrnehmung übernimmt: Die meisten Menschen gehen nämlich bei der Bewältigung ihres Alltages implizit von einer gegebenen Freiheit aus (vgl. DREHER 1987: 1). Sie wägen Alternativen ab, treffen Entscheidungen und weisen Verantwortung für gewählte Optionen zu. Die Vorstellung, keineswegs frei zu sein in den Entscheidungen, ruft in der Regel Unbehagen hervor. Jede Form der Einschränkung im Sinne eines Determinismus vergangenen, gegenwärtigen oder zukünftigen Verhaltens wird eher kritisch gesehen. Ein simples Beispiel mag dies illustrieren: eine einfache Kaufentscheidung für ein beliebiges Produkt in einem beliebigen Geschäft. Wie die meisten Handlungen wird auch dieser Einkauf als frei wahrgenommen. Etwaige einschränkende Randbedingungen wie der Preis, die Wirksamkeit von Werbemaßnahmen, die Bedeutung des Produkts für den Käufer, die verfügbaren Ressourcen oder Alternativen in der Auswahl werden nicht als ultimativ bestimmend angesehen. Stattdessen entsteht der Eindruck, dass man sich ja frei entscheiden oder natürlich auch den Kauf nicht oder anders durchführen könne.

Diese Kombination aus scheinbar freier Entscheidung und dahinter verschwindenden begrenzenden Faktoren ist eine Sichtweise, die z. B. für das 
Justizwesen gültig ist. Vorstellungen wie Schuld und Strafe nehmen freien Willen als Voraussetzung an. Wäre ein abweichendes Verhalten vorherbestimmt, so könnte man es jenseits von Rachegedanken nicht in sinnvoller Weise sanktionieren. Etwaige Einfluss nehmende Randbedingungen werden vor allem bei der Berücksichtigung von Schuld- und Zurechnungsfähigkeit mit berücksichtigt, obgleich im Regelfalle Verantwortung dem Täter zugeschrieben wird, hatte er doch die Wahl, die Tat zu begehen oder zu unterlassen.

Die folgenden Unterabschnitte versuchen, der skizzierten Problematik gerecht zu werden, indem ein eigenes Konzept von Freiheit vorgestellt wird, das auf der Diskussion über den Determinismus von Handlungen und einem zusammenfassenden Modell gängiger Hauptbedeutungen von Freiheit aufbaut.

\subsubsection{Eine Typologie von »Freiheit»}

Eine umfassende Darlegung der Begriffsgeschichte von Freiheit soll hier nicht gegeben werden, da im Folgenden bei der Diskussion des Determinismusproblems die im Kontext wichtigsten Inhalte aufgegriffen werden. Vielmehr soll in diesem Abschnitt eine zusammenfassende Darstellung des Freiheitsbegriffs einer empirischen Grundlagenstudie zum Freiheitsverständnis der Deutschen (Titel: „Der Wert der Freiheit«) des Instituts für Demoskopie Allensbach (IfD) gegeben werden (vgl. IfD 2003). Die verschiedenen Dimensionen und Vorstellungen von Freiheit wurden bei der Untersuchung theoretisch erarbeitet und dann in einer Befragung überprüft und veranschaulicht. Sie geben einen guten Überblick über die verschiedenen Bedeutungsdimensionen des Begriffs und dienen als erster Erarbeitungsschritt. 
Insgesamt werden fünf verschiedene Bedeutungen von Freiheit angegeben (ebd.: 17f.), die durch die anderen methodischen Schritte der Studie weiter verfolgt wurden:

- Älteste Bedeutung: Freiheit von Willkür und Sklaverei: libertas;

- Möglichkeit, zu tun und zu lassen, was man will: libertinage;

- Freiheit von Not, Armut, Arbeitslosigkeit, von allen möglichen Risiken des Lebens;

- Freiheit im politischen Sinn;

- Lebensgestaltung, Verantwortungsübernahme, Suche nach Erfolg.

Die älteste Bedeutung des Begriffs erklärt sich von der gegensätzlichen Position her. Das Gegenstück zu Freiheit in diesem Sinne ist Sklaverei und Unterjochung. Somit ist nach GRIMM (zitiert nach ebd.) der gotische Ausdruck »Freihals« eben der »Hals, der kein Joch tragen mußte» (ebd.). Die Autoren der Studie verweisen darauf, dass diese »Kernbedeutung von Freiheit» (ebd.) sich - wenn auch in unterschiedlichem Maße - in allen anderen Bedeutungszusammenhängen wieder findet.

Die zweite Bedeutung von Freiheit ist gestaltungsorientiert, sie betrifft das Ausleben und die freie Entfaltung, »im Extremfall ohne Rücksicht auf die Bedürfnisse anderer und ohne Verantwortung und Verpflichtungen« (ebd.: 18). Diese schrankenlose Freiheit ist vermutlich nur bei eremitär lebenden Menschen möglich und diejenige, die am schwersten in der Realität zu finden ist, da davon auszugehen ist, dass die ausgelebte Freiheit schnell Berührungsund Konfliktpunkte mit anderen ergeben wird. Somit ist zu erwarten, dass diese schrankenlose Freiheit unter bestimmten Umständen mit der Freiheit der Mitmenschen kollidieren wird. 
Die dritte Bedeutung von Freiheit im Sinne von Befreiung von materieller Not und von Risiko wird von den Autoren der Studie als am weitesten von der ältesten Bedeutung entfernt gesehen (vgl.ebd.). Mit dem Hinweis auf einen starken Staat, der diese Freiheit gewähren und sichern muss, wird diese Freiheit in die Nähe von vor allem sozialistischen Diktaturen gerückt und teilweise sogar im Widerspruch zur eigentlichen Bedeutung gesehen (vgl. ebd.). Dies muss aber nicht zwangsweise so sein. Tatsächlich ist die Übertragung an den Staat nicht unbedingt erforderlich, da im Sinne von Subsidiarität auch kleinere soziale Einheiten wie beispielsweise Familien oder größere Familienverbände diese Art der Freiheit tendenziell oder total ermöglichen könnten. Man kann in kleinerem Rahmen diese Form der Freiheit auch als Grundstock und Basis für die weitere Entfaltung der anderen Bedeutungsvarianten von Freiheit sehen. Nur mit einer materiellen Absicherung in einem bestimmten Umfeld ist libertas sinnvoll und möglich, auch libertinage ließe sich leichter auf der Basis einer ökonomischen Absicherung und bei Absenz von Risiko vollziehen.

Als Freiheit im politischen Sinn bezeichnet die Studie »die Möglichkeit, als Bürger ungehindert am öffentlichen Leben teilzunehmen« (ebd.). Dies umfasse sowohl die Möglichkeit zur aktiven und passiven Teilnahme an demokratischen Wahlen als auch das Recht auf freie Meinungsäußerung und »weitgehend ungehindert(en)« (ebd.) Zugang zu Informationen über Politik. Wichtig ist hierbei, dass nicht der tatsächlich realisierte Zugang zur Information oder die tatsächlich erfolgte freie Meinungsäußerung gemeint sind, sondern vielmehr die Möglichkeit, also das Potenzial, politisch entsprechend partizipieren zu können. Es stellt sich die Frage, ob dieses bloße Potenzial genügt oder ob realiter auch politisch gehandelt werden muss, um Freiheit in diesem 
Kontext zu praktizieren. Wird eine Gesellschaft weniger frei, wenn ihre Mitglieder nicht am politischen Leben teilhaben? Oder kann man frei nach Thomas Morus davon ausgehen, dass Schweigen zustimmen heißt (vgl. CHAMBERS 1947: 207)?

Grundsätzlich spielt bei einer korrekten Einordnung der politischen Partizipation auch der zeitliche Rahmen eine Rolle. Politische Inaktivität führt nicht sofort und nicht notwendig zum Verlust von Freiheit, kann ihm aber langfristig den Weg bereiten. Andererseits kann man den Vorgang der Nichtteilhabe tatsächlich als absichtliche Verweigerung aus bewussten Motiven heraus interpretieren. Bewusstes politisches Unterlassen kann somit ebenfalls eine Form des freien politischen Handelns sein, zumindest wenn die Voraussetzung erfüllt ist, dass der Betreffende ausreichendes Wissen besitzt und sich informieren kann. Nur in diesem Falle erscheint es als wahrscheinlich, dass die Nicht-Partizipation als bewusstes, freies politisches Handeln gelten kann.

Informationen besitzen aber nicht nur im Kontext der Partizipation eine besondere Brisanz. Durch Filterung, einseitige Berichterstattung oder gezielte Falschinformation kann Freiheit im politischen Sinn eingeschränkt werden, ohne dass es dem Einzelnen - mangels kompetenter Informationsalternativen oder durch das bloße Fehlen eines Gespürs für falsche Information - wirklich bewusst ist. Es besteht also die Gefahr, fehlgeleitet zu werden und so politische Freiheit durch Manipulation zu verlieren.

Das zuletzt genannte Verständnis von Freiheit wird von den Autoren als das "fundamentalste und sozialwissenschaftlich bedeutendste» (ebd.) bezeichnet. Freiheit wird verstanden «als die Möglichkeit des einzelnen, sein Leben selbst in die Hand zu nehmen, aktiv den Erfolg im Leben zu suchen und zu gestalten, Chancen zu ergreifen, für sich selbst Verantwortung zu überneh- 
men, einschließlich der Möglichkeit zu scheitern und dann auch die Folgen zu tragen« (ebd.). Diese Definition von Freiheit, so weiter, sei ein "gesellschaftliches Grundprinzip« (ebd.), das für viele politische und gesellschaftliche Entscheidungen gelte. In jedem Falle ist der Kern der Freiheit hier die Handlungsfreiheit. Sie meint aber nicht nur das Entscheiden zwischen gegebenen Alternativen, sondern umfasst darüber hinaus auch das Entstehen der Entscheidungssituation selbst, das sich kreativ und gestaltend durch den Einzelnen vollzieht. Diese Freiheit in den Entscheidungssituationen bedeutet auch für die wahrgenommene Option verantwortlich zu sein: »No pratical definition of freedom would be completely without the freedom to take the consequences. Indeed, it is the freedom upon which all others are based" (Pratchett 2004: 15). Es ist in der Tat bei diesem Verständnis des Begriffs bedeutsam, auch das Scheitern des freien Handelns mit einzubeziehen. Die Freiheit, die Folgen zu tragen, ist hier unmittelbar gegeben, da diese wieder neue Entscheidungssituationen kreieren, die ihrerseits wieder bewältigt werden müssen.

Die Studie beinhaltet nach einer ganzen Reihe von verschiedenen Frageformen zur Bedeutung des Begriffs Freiheit auch einen Test, bei dem die Befragten 25 Karten mit Stichworten zu Aspekten von Freiheit nach drei Stapeln ordnen sollten. Die drei Stapel symbolisierten die Relevanz des Einzelnen bezüglich des jeweiligen Aspekts: »Habe unbedingt mit Freiheit zu tun«, »habe auch noch mit Freiheit zu tun«, »habe nichts mit Freiheit zu tun«. Es ergab sich die folgende aufschlussreiche Übersicht (siehe Abbildung 1): Die Autoren der Studie interpretieren die Ergebnisse dieser Fragestellung, indem sie die einzelnen Items, den einzelnen Bedeutungen des Freiheitsbegriffs zuordnen. Meinungsfreiheit, Freizügigkeit, das Recht auf freie Arbeitsplatzwahl 


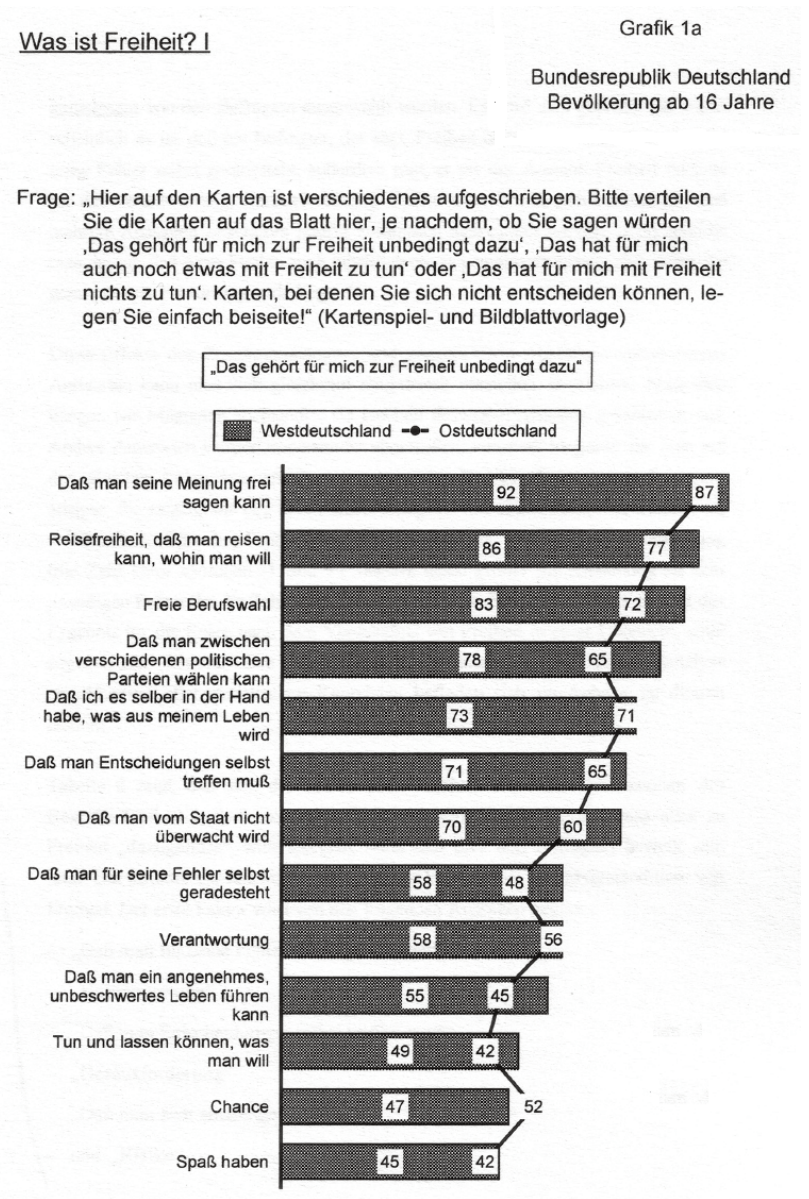

Abbildung 1 Was ist Freiheit? (IFD 2003: 32)

und die Möglichkeiten für parteipolitische Alternativen belegen die Plätze eins bis vier. Sie werden zu den politischen und rechtlichen Aspekten gezählt (IfA 2003: 32). Die fünf darauf folgenden Rangplätze werden von Aussagen belegt, die im Sinne von Entscheidungsfreiheit verstanden werden. Auf diese folgen ab Platz zehn Aussagen, »die auf ein Verständnis von Freiheit im Sinne von 
Freiheit von Not und sozialer Benachteiligung schließen« (ebd.) lassen. Auffallend ist bei den Ergebnissen, dass nur beim vorletzten Item »Chance« der ostdeutsche Wert den westdeutschen übersteigt, was aber nicht ausführlicher in der Studie diskutiert wird. Ebenfalls fällt auf, dass die in der Studie angeführte dritte Bedeutungsdimension von Freiheit im Sinne von »Freiheit von Not, Armut, Arbeitslosigkeit, von allen möglichen Risiken des Lebens« (siehe Seite 15) außen vor gelassen wird und entsprechende Inhalte nicht zugeordnet werden können.

Zuletzt sei noch erwähnt, dass die Studie auch auf die oft gebrauchte Unterscheidung von Freiheit zu etwas und Freiheit von etwas eingeht und diese als »nicht geeignet« bezeichnet (ebd.: 19). Dieser Kritik könnte man sich insofern anschließen, ginge man davon aus, dass diese Unterscheidung im Kontext dieser fünf Bedeutungen tatsächlich nicht zu mehr Einsicht und Verständnis beitrüge, zumindest wenn sich klare Gegensatzpaare bilden ließen: Die Freiheit von Vorschriften und Zwängen ist immer auch Freiheit zu mehr Handlungsspielraum und Alternativen. Freiheit von Hunger und Not ist ebenfalls als Freiheit zu ausreichender Versorgung und zu einem sorgenfreien Leben denkbar. Insofern ergibt die Unterscheidung in Freiheit von oder Freiheit zu keine weitergehende Klarheit, sondern erscheint tatsächlich eher als redundant. Ein genauer Blick auf die Unterscheidung zwischen positiver und negativer Freiheit lohnt sich aber dennoch. Zurückführen lässt sich die begriffliche Unterscheidung auf KANT, der in seiner Grundlegung zur Metaphysik der Sitten Freiheit als »Eigenschaft des Willens aller vernünftigen Wesen« (KANT 2000: 455) voraussetzt und an gleicher Stelle zwischen "positiver « und »negativer« (ebd.: 454) Freiheit differenziert. BERLIN greift diese Unterscheidung auf und veranschaulicht sie unter anderem für den Bereich Politik: 
»Gewöhnlich sagt man, ich sei in dem Maße frei, wie niemand in mein Handeln eingreift, kein Mensch und keine Gruppe von Menschen (Berlin 1995: 201).« Unfreiheit im Hinblick auf diese negative Freiheit ergibt sich da, wo andere das politische Handeln behindern und den Einzelnen damit an der Ausübung hindern.

Dem gegenüber leitet BERLIN positive Freiheit aus dem Wunsch des Individuums ab, »sein eigener Herr« (ebd.: 211) zu sein und erweitert sie um das tatsächliche Wahrnehmen der Möglichkeiten der freien Handlungsmöglichkeiten aus eigenem vernünftigen Antrieb und unabhängig von äußeren Einflussnahmen (ebd.: 211) und somit um eine Aktivierung der passiven negativen Freiheit.

Insgesamt betrachtet vermittelt diese Studie einen guten Überblick über alltägliche Vorstellungen von Freiheit. Es darf angenommen werden, wie ja auch die Zuordnung der verschiedenen Items auf den 25 Karten zu den jeweiligen Bedeutungen, dass sich die meisten alltäglichen Vorstellungen von Freiheit einordnen lassen. Trotzdem wird die entscheidende Frage im Vorfeld nicht gestellt, nämlich, ob prinzipiell freies Handeln überhaupt möglich ist und wenn ja, wie weit diese Freiheit reicht. In der Tat muss man sich der Beantwortung dieser Frage stellen, will man im Rahmen dieser Analyse zu aussagekräftigen Ergebnissen kommen. Die axiomatische Akzeptanz der Existenz von Freiheit vereinfacht das Thema unzulässig, da bestimmte Bereiche des Begriffsspektrums von Freiheit a priori ausgeblendet werden und somit eine angemessene Betrachtung verhindern. In den folgenden Abschnitten soll nun der Blick weg von alltäglichen Bevölkerungsvorstellungen von Freiheit auf tiefergehende Problematiken gerichtet werden, wobei besonders der Willensfreiheit und dem Determinismus die größte Aufmerksamkeit gilt. 


\subsubsection{Handlungs-Determinismen und die Willensfreiheit}

»L'homme est né libre, et par-tout il est dans les fers.« Dieser bekannte Satz aus Rousseaus Contrat Social (1964: 351) macht auf ein grundsätzliches Problem aufmerksam, das sich auf mehreren Bedeutungsebenen verfolgen lässt. Der frei geborene Mensch ist bei RousseAu eine spekulative Annahme, der er zunächst nicht weiter argumentierend folgt. Stattdessen sucht er nach Möglichkeiten, die angeborene Freiheit, die er in Ketten geschlagen und somit verloren sieht, wieder zu erlangen. Der Mensch findet sie letztlich wieder in einem besonderen Gemeinwesen, in dem der Einzelne durch den Zusammenschluss in der Gemeinschaft geschützt wird und doch so frei bleibt wie vor dem Eintritt (Rousseau 1986:17) in dieselbe. Somit steht dieser Satz auch für zwei alternative Entwicklungen von Freiheit: Einmal endet sie in Ketten, ein anderes Mal steht am Ende erneut die Freiheit, umgesetzt im Gemeinwesen. Die Ketten, um im Bild zu bleiben, sind bei Rousseau ein Symptom eines HerrSklave-Verhältnisses, das allerdings beide bindet (ebd.) und letztlich verschwimmen lässt, wer Sklave und wer Herr ist. Man kann aber über dieses reine Machtverhältnis hinausgehen und sich weitergehend und umfassend mit den Ketten beschäftigen, die den Menschen seiner Freiheit berauben. Letztlich kann man durchaus dieses Bild beibehalten, wenn man von Determinismen spricht, die den Menschen in seinem Handeln begrenzen und bedingen.

Die Diskussion, ob der Mensch fremdbestimmt beziehungsweise determiniert oder frei (indeterminiert) ist, lässt sich nach verschiedenen wissenschaftlichen Fachbereichen ordnen, wobei die Ansätze sich zum Teil gegenseitig beeinflussen. Während die maßgeblichen soziologischen Ansätze im folgenden Abschnitt 2.1.3 näher behandelt werden, stehen zunächst die 
folgenden Disziplinen im Mittelpunkt, da sie die soziologische Perspektive erheblich bereichern können:

- Philosophie;

- Theologie;

- Physik;

- Biologie.

Die Philosophie beschäftigt sich schon seit langem mit der Frage nach der Determiniertheit und der Willensfreiheit des Menschen. Gerade in der Erkenntnistheorie und auch in der Ethik spielt das Thema eine entscheidende Rolle. Bei Letzterer stellt sich über die Freiheit hinaus vor allem die Frage nach der Verantwortung für das Handeln des Einzelnen. Fiele die Freiheit weg, so hätte auch die Verantwortung keinen Platz; sie wäre einfach den determinierenden Bedingungen geschuldet und daher irrelevant. Es würde den Rahmen sprengen, das Thema aus der Sichtweise sämtlicher Philosophen zu schildern, die teilweise als Deterministen oder als Anhänger der Willensfreiheit auftraten (vgl. DREHER 1987: 63). Stattdessen soll hier eine Auswahl getroffen werden, welche die wichtigsten Gedanken wiedergibt.

Ein wichtiger Kernpunkt ist dabei der Begriff der Kausalität, der aussagt, dass es zu jedem Vorgang eine oder mehrere Ursachen gibt, die denselben bedingen. ARIStOtEles unterscheidet für jegliches Geschehen neben einer generellen Unterteilung in innere und äußere Ursachen in Formursache, Materialursache, Antriebs- oder Wirkursache und End- oder Zweckursache (vgl. WUKETITS 1981: 20). WUKETITS benennt in seinem Überblick das häufig rezipierte Beispiel des Hausbaus. Nach der aristotelischen Einteilung repräsentieren die Baupläne die Formursache, das Baumaterial die Materialursache, 
der Bau des Hauses an sich Wirkursache und letztlich die Intention des Bauherrn die Zweckursache (vgl. ebd.). Diese Position von ARISTOTELES bezieht sich in der Regel auf beobachtbare Phänomene: Ein Phänomen X wird als Ursache eines Phänomen $Y$ angenommen, $Y$ ist somit die Wirkung von $X$. Die Ursache kann gemäß der vier genannten Kriterien aufgeschlüsselt werden. Das beobachtbare, zeitliche Zusammenfallen von Ursache und Wirkung kann in die Irre führen und lediglich eine Scheinkausalität sein, deren wahre Natur verborgen bleibt. Dies gilt vor allem für Handlungen, die man als Außenstehender in puncto Kausalität betrachtet und bewertet (vgl. ebd.: 17): Beobachtbare Korrelationen zwischen bestimmten Ereignissen weisen noch keine kausalen Wirkzusammenhänge nach.

Der englische Philosoph David Hume erweitert das Problem des kausalen Zusammenspiels aus beobachteter Ursache und Wirkung, um den Aspekt der Wiederholbarkeit, das »nachfolgende Philosophiegenerationen« (PRECHTL 1995: 410) herausfordern sollte. Eine mehrfache Beobachtung der gleichen Abfolge von Ereignissen sagt nämlich nach HUME rein logisch ebenfalls nichts über den Wahrheitsgehalt des Zusammenhangs von Ursache und Wirkung aus. Dieses Phänomen ist durch Karl Popper (vgl. Popper 1934: 9) als das so genannte HumE'SCHE Problem im Rahmen des Kritischen Rationalismus aufgegriffen worden und wurde als »Induktionsproblem« (WUKETITS 1987: 27) der Erkenntnistheorie zum populären Begriff. Abseits der wissenschaftlichen Diskussion wenden im Alltag viele Menschen die logisch nicht beweisbare Prognosemöglichkeit an und schließen relativ unbekümmert aus vorhergehenden Phänomenen analog auf zukünftige Ereignisse. Korrelationen von verschiedenen Ereignissen werden hier ganz zwanglos als echte Kausalität interpretiert. 
Die Kausalität ist ein wesentlicher Aspekt des Determinismus. Lassen sich für jedes Ereignis eine oder mehrere Ursachen feststellen, so stellt sich vor allem die Frage, ob es Freiheit in Hinblick auf Libertinage und Lebensgestaltung überhaupt gibt. Es bleibt ungeklärt, ob es ein freies Subjekt gibt, das eine Kausalkette aus nachgeordneten Wirkungen unabhängig und bewusst starten kann. Hinweise auf eine Lösung dieses Problems finden sich bei DESCARTES und KANT. Ersterer ging von einem Dualismus von res cogitans (Geist) und res extensis (Dingwelt) aus. Diese theoretische Denkfigur bedeutete letztlich eine Entkoppelung des menschlichen Geistes von der Materie, für die DescARTES aber durchaus Ursache-Wirkungs-Zusammenhänge annahm. Freie Entscheidungen werden durch den Dualismus erst möglich, da der menschliche Körper als Teil der mechanisch funktionierenden Natur beschreibbar durch die Geometrie als Teil der Mathematik (vgl. RöD 1964: 131) - determiniert ist. Der menschliche Geist kann sich im Gegensatz dazu frei von materiellen Schranken unabhängig entfalten.

IMMANUEL KANT liefert in seiner »Kritik der praktischen Vernunft« einen ähnlichen Ausweg aus dem Problem der Willensentscheidungen. Auch er versteht unter Freiheit »das Vermögen, einen Zustand von selbst anzufangen« (KANT 2000: 528). Dies wird erst ermöglicht durch die Vernunft (vgl.ebd.: 549) und den Verstand, der seinerseits das durch die Sinne Wahrgenommene strukturiert (vgl. JONAS 1972: 43) und freien Willen so ermöglicht. Das entscheidende Merkmal des Menschen ist seine Möglichkeit, abseits zwanghafter Kausalität frei, das heißt autonom und nach dem eigenen Willen, zu entscheiden.

Eng mit den philosophischen Entwicklungen verbunden sind Fragestellungen, die sich im Bereich der Theologie bezüglich des Determinismus ergeben. Sie stellen sich dabei religionsübergreifend und einen in diesem Bereich die 
verschiedenen Religionen. Jede Vorstellung von göttlichen Entitäten geht in der Regel von bestimmten Omnipotenz- und Allwissenheitsvorstellungen aus. Aus dieser göttlichen Konstellation heraus ergibt sich eine bestimmte Schicksalsvorstellung, die von Elementen wie Vorsehung oder Schicksal geprägt ist. Als vorbestimmte, wenn auch unergründliche Wege des Herrn manifestieren sich diese Vorstellungen im Christentum, ähnliche Überzeugungen kennt beispielsweise auch der Islam mit dem Begriff „Kismet« (KNIESCHKE 1916: $35 \mathrm{ff}$ ), der Verwandtes meint. In jedem Fall hat der angenommene Einfluss Gottes weit reichende Auswirkungen auf die menschliche Willensfreiheit, die dann nur schwer Raum mehr findet. Für den Gläubigen stellt sich die Frage nach der Verantwortung, die in der oben skizzierten Argumentation allein bei Gott liegt, da er den Lebensweg der Menschen und damit alle positiven und negativen Bereiche vorzeichnet. Christliche Theoretiker bemühen sich bis heute, die Allmacht Gottes mit der Freiheit für den Menschen zu verbinden (vgl. DREHER 1987: 157 f.), was sich aber in der Praxis als schwieriger Gegensatz erweist.

Die Physik liefert Pro- und Contraargumente bezüglich der Willensfreiheit. Die Grundlage aller physikalischen Theorie sind »Maßaussagen« (CASSIRER 1937: 40), die Wahrnehmungsdaten in physikalische Einheiten übersetzen. Darauf aufbauend sucht die Physik nach Gesetzen, welche die beobachtbaren Abläufe erklärbar und verständlich machen sollen. Ein prominentes Beispiel dafür ist die NewTON'SCHE Festkörperphysik, die bestimmte Gesetzmäßigkeiten der Planetenbewegungen nachzuweisen versuchte und erstmals die Schwerkraft näher charakterisierte. Die alltägliche Erfahrung von physikalischen Vorgängen wie der Wirkung der Schwerkraft, beispielsweise wenn ein beliebiges Objekt zu Boden fällt, verstärkt für den Beobachter den Eindruck 
von Kausalität und Unvermeidlichkeit. Die im Zeitalter von NEwTON tätigen Physiker und die neu entdeckten Gesetzmäßigkeiten von physikalischen Phänomenen verstärkten den Eindruck von Unausweichlichkeit und Determination. LAPLACE beispielsweise entwickelte in diesem Zusammenhang ein mechanistisches Weltbild, in dem einem Uhrwerk gleich Ursache und Wirkung lückenlos ineinander greifen (vgl. DREHER 1987: 182). Ein gewaltiger Paradigmenwechsel in der Physik durch PLANK und EINSTEIN zerstörte die deterministischen Vorstellungen gründlich. NEWTONS Theorie von den Planetenbewegungen scheiterte an astronomischen Beobachtungen der Monde des Merkurs (vgl. Pratchett/Stuart/Cohen 1999: 79). Einstein füllte die entstehende theoretische Lücke mit der allgemeinen und der speziellen Relativitätstheorie, die im Grunde keinen besonders geglückten Namen erhielt. Es ist nämlich in der Tat nicht so, dass in der EINSTEIn'SCHEN Physik alles relativ ist, sondern gerade die Lichtgeschwindigkeit als elementarer Bestandteil der Theorie ist absolut konstant und somit unveränderlich. Neu aber ist die Tatsache, dass physikalische Vorgänge nicht mehr absolut gleich ablaufen ${ }^{1}$, sondern vom Beobachter abhängen. Raum und Zeit werden zu Variablen und beenden die umfassende Gültigkeit des mechanistischen newton'schen Weltbilds. Die Vorstellung eines durchgängigen Kausalgesetzes war mit der neuen Physik nicht haltbar. Dies machte auch die wissenschaftliche Leistung HEISENBERGS

1 Das so genannte Zwillingsparadoxon illustriert genau diese Abhängigkeit von einem Beobachter. In einem Gedankenexperiment wird von Zwillingen ausgegangen, von denen in einem gedanklichen Experiment einer auf der Erde verbleibt, während der andere sich mit annähernd Lichtgeschwindigkeit in einem Raum altern beide in unterschiedlichem Umfang (vgl. u.a. bei SEXL/SCHMIDT 2000 und TIPLER/LLEWELLYN 2003). Zwei Menschen, die identisch alt sein müssten, sind es durch die Bewegung im Raum nicht mehr. 
deutlich, der auf Teilchenebene nachweisen konnte, dass exaktes Messen von subatomaren Teilchen nicht möglich ist (Unschärferelation). Ausgehend von der so genannten Doppelnatur von Elektronen - sie verhalten sich sowohl als Welle als auch als subatomare Teilchen - konnte er darlegen, dass ihre genaue Ortsbestimmung nicht möglich ist, da die Messung bereits den Ort der Teilchen verändert. Eine umfassende Darstellung, wie die genannten mikrophysikalischen Beobachtungen auf den makrophysikalischen Raum übertragen wurden, findet sich bei DreHER (1987: $200 \mathrm{ff}$.). Hier bleibt abschließend festzuhalten, dass der Paradigmenwechsel in der Physik einen starren, umfassenden Determinismus ausschließt. Kausale Zusammenhänge werden aber nicht grundsätzlich negiert, sondern werden prinzipiell und nicht ausschließlich denkbar. Freiheit im Sinne von Willensfreiheit ist durch die neuen Freiräume der Physik nicht bewiesen, gleichwohl wird zumindest innerhalb dieser wissenschaftlichen Disziplin der Möglichkeit für Freiheit abseits starrer Determinismen Raum und Möglichkeit gegeben.

Die Entwicklungen in der Biologie hatten ebenfalls Auswirkungen auf das Verständnis von Determinismus und Freiheit. Wichtig sind dabei die Evolutionstheorie, die Genetik und die Ethologie. Die auf DARWIN und den oftmals zu Unrecht ignorierten WALLACE (vgl. WALLACE 1871) zurückgehende Evolutionslehre, die DARWIN in seinem Werk »The Origin of Species« ausformulierte, ist dabei der entscheidende Ansatz. Unter dem Schlagwort natural selection versteht DARWIN ein Prinzip für die Entwicklung der Lebewesen, das sich durch die Weitergabe verschiedener Merkmale an die nächste Generation vollzieht. Dies gilt für bestimmte Anlagen, deren phänotypische Ausprägung einen Vorteil für die nächste Generation von Lebewesen bedeutet. WATSONS und CRICKS Entdeckung der speziellen Helixstruktur der DNS ergänzte das Prinzip 
der natürlichen Auswahl um die chemischen Grundlagen. Der aus nur vier Basen bestehende chemische Code der DNS liefert gewissermaßen in Form der verschiedenen Chromosomen die Blaupausen für die Synthetisierung verschiedener Proteinstrukturen, aus denen sich die einzelnen Bestandteile des menschlichen Körpers aufbauen. Durch die Beteiligung zweier Partner bei der Fortpflanzung kommt es zur Aufspaltung der DNS-Doppelhelix und durch das sich anschließende neue Zusammensetzen der verschiedenen Einzelstränge zur Rekombination der Gene, die zusammen mit zufällig verlaufenden Mutationen zu neuen phänotypischen Merkmalsausprägungen führen. Diese neuen Merkmale unterliegen dann dem Praxistest der natürlichen Auswahl und können sich dann durchsetzen - oder eben nicht. Auf Basis dieser Überlegungen stellt jeder der heute lebenden Organismen vom Einzeller bis zum Wirbeltier eine evolutionäre Erfolgsgeschichte dar, unabhängig vom biologischen Organisationsgrad. Die Tatsache, dass bestimmten Gensequenzen bestimmte Merkmalsausprägungen zugewiesen werden können, stützt bis heute die Vorstellung, es gäbe eine Art biologischen Determinismus. Die Gene würden aus dieser Perspektive zu Determinanten; sie würden bestimmte geistige und körperliche Eigenschaften der Menschen festlegen und damit auch auf das Verhalten massiv einwirken. Insbesondere im Bereich von numerischen Chromosomenaberrationen wurden bestimmten Kombinationen wie etwa die XYY-Konstellation bei den Geschlechtschromosomen mit gewissen aggressiven Neigungen und Kriminalität in Verbindung gebracht. Gerade bei letzterer relativieren allerdings neuere Veröffentlichungen den von NIELSON (et al.) angenommenen, und in viele Lehrbüchern unüberprüft übernommenen, direkten Zusammenhang zwischen der genetischen Konstellation und der Neigung zur Delinquenz (u. a. GünTHER 1984: 273 und WiEDEMANN/ 
KUnZe/SPRANGER 2001: $96 \mathrm{f})^{1}$. Neben physischen Merkmalsausprägungen aufgrund von DNS, versuchten Ethologen wie KONRAD LORENZ auch Verhaltensweisen bestimmter Tiere (vgl. ANHÄUSER 2006) in das Gedankengebäude der genetischen Vererbung zu integrieren. Die Erhaltung der eigenen Art stehe im Mittelpunkt, somit seien Verhaltensweisen wie etwa die Jungentötung von Löwen, die ein Rudel übernehmen, als unnatürlich einzustufen. Der Biologe DAWKINS (1978) hinterfragte das gängige Lehrmodell der Arterhaltung und stellte seinerseits das »egoistische Gen« in den Mittelpunkt seiner Überlegungen. Das Beispiel der Jungentötung erschien aus dieser Sicht auf einmal als sinnvoll. Der neue Rudelführer konnte so sicher sein, dass nur die eigenen Gene weitergegeben werden. DAWKINS Äußerungen über den Menschen sorgten ebenfalls für Aufsehen: »Wir sind nur die Überlebensmaschinen der Gene. Wenn wir unseren Zweck erfüllen, werden wir beiseite geschoben« (DAWKINS, zitiert nach ANHÄUSER 2006). Diese plakative Formulierung polarisierte die Meinungen und schien einem weit reichenden Determinismus nach dem Munde zu reden. Der handelnde Mensch ist demnach von seinem Genom gesteuert, die Weitergabe der Erbinformationen ist der alleinige Zweck allen menschlichen Verhaltens. Trotzdem lässt diese Sichtweise Platz für freie Entscheidungen, da Menschen auf vielfältige Weise sich gegen die Weitergabe von Erbinformationen sperren können. Dazu kann man bestimmte Formen der Empfängnisverhütung, der Verzicht auf Sexualität, Selbstmord oder jegliche Form von das Erbgut schädigenden Verhaltensweisen, wie etwa das

1 Im Mittelpunkt der Kritik an NIELSON und seinen Mitautoren standen statistische Mängel und davon abgeleitete unzulässige Schlussfolgerungen bezüglich der Intelligenz und Delinquenz von XYY-Männern. 
Rauchen, zählen. Offensichtlich können Gene auch scheinbar erratisch funktionieren oder zumindest können sich die Menschen als ihre Transportvehikel kontraproduktiv verhalten.

Im Bereich der Biologie gibt es z. B. durch den Hirnforscher WoLf SINGER neue deterministische Ansätze, die sich auf den Bereich der Hirnfunktionen beziehen. In einem Zeitungsinterview führt SINGER sämtliche Entscheidungen des Menschen auf neuronale Prozesse in der Großhirnrinde zurück (vgl. DRACH 2006). Jedes Gefühl, Emotion, kognitive Leistung oder jede wahrgenommene soziale Realität benötigte eine physische Voraussetzung, die sich im Gehirn manifestiert (vgl. ebd.). Freie Entscheidungen, so SINGER weiter, seien nur dann gegeben, wenn sie nach dem Abwägen von Argumenten, möglichst unbeeinflusst von äußeren Einflüssen (etwa Hirnschädigungen oder Drogen) und auf der bewussten Ebene des Gehirns getroffen werden. Die Argumente würden auf soziokulturellem Wissen beruhen, nach der Entscheidung wird diese als frei bezeichnet und der Handelnde wird »voll verantwortlich gemacht« (DRACH 2006.). Die eigentlichen Denkvorgänge würden aber nur als frei gefühlt, tatsächlich basiere das gesamte Denken auf »neuronalen Erregungsmustern« (DRACH 2006.), die versuchen, möglichst in sich stimmig zu sein. Die Leistung des Gehirns zur Perspektivenübernahme und die Fähigkeit, abwägen zu können, resultierten in einem Ich-Gefühl, hinter dem die vielfältigen neuronalen Determinanten verborgen blieben. Das Phänomen, dass Veränderung der Gehirnstrukturen etwa durch Schädigungen nach einem Unfall - als klassisch gilt in diesem Kontext der Fall PHINEAS GAGE (ausführlich dazu u.a. bei DAMASIO 1994: 25-39) - die Persönlichkeit grundlegend verändern können, ergänzt die obige Perspektive SINGERS. Der menschliche Geist wird damit theoretisch endgültig wieder mit dem Körperlichen 
verschränkt. Neben den Veränderungen, die Gehirntraumata auslösen können, gilt Ähnliches auch für Tumore, deren Wachstum ebenfalls Persönlichkeitsmerkmale modifizieren kann (vgl. MARKOWITSCH 2006: 33). Die neurobiologische Perspektive SINGers ergänzt sich mit der klassischen Genetik, die man weit verbreitet in alltäglichen Zeitungspublikationen, aber auch in gängigen Schulbüchern vorfindet. Doch wie so oft ergeben sich durch vereinfachte Darstellungen und Verallgemeinerungen schlichtweg Irrtümer. Dies gilt auch für die Genetik, denn vor allem die Steuerung von bestimmten Merkmalsausprägungen durch genetische Sequenzen ist noch keineswegs umfassend erforscht. Enorme Schwierigkeiten machen den Biologen die hohe Übereinstimmung von Gensequenzen von verschiedenen Arten, wo nur quantitativ geringfügige Unterschiede in der DNS zu höchst unterschiedlichen physischen Erscheinungen führen können. Ebenfalls ungeklärt sind Ursachen für die Aktivierung bestimmter Gensequenzen, die zu anderen Zeiten schlafend inaktiv sind und keine feststellbare Funktion haben.

Neuere Forschungsansätze untersuchen die Auswirkungen von Lebenserfahrungen und Umwelteinflüssen auf den individuellen genetischen Code. Bei traumatisierten Patienten und in verschiedenen Tierversuchen konnte nachgewiesen werden, dass bestimmte Erlebnisse zu biochemischen Veränderungen der molekularen Verpackung der DNS und weiter zu organischen Veränderungen führen (vgl. KASTILIAN 2008). Bestimmte Gensequenzen werden dabei blockiert und damit die Funktion des Genoms modifiziert, wobei angenommen wird, dass diese Veränderungen auch weiter vererbt werden. Obwohl diese Forschungsansätze noch relativ neu und auch noch größtenteils auf Tiermodellen beruhen, zeigt sich doch ein gewisser Perspektivenwechsel ab. Das Genom wird aus dieser Perspektive nicht mehr als starrer Bauplan betrachtet, 
sondern als durchaus veränderbare Skizze, die auch innerhalb einer Generation Veränderungen unterworfen sein kann.

Die Frage nach der individuellen Freiheit des autonomen Individuums ist offenkundig auch unter Einbeziehung verschiedener wissenschaftlicher Disziplinen nicht eindeutig oder gar leicht zu beantworten. Es gibt Ansätze, welche die These der Freiheit zu stützen scheinen und andere, die den Determinismus leichter vorstellbar machen. Der folgende Abschnitt möchte eine soziologische Antwort auf das Problem geben und versucht, weitere Ansätze zu integrieren.

\subsubsection{Eine soziologische Sichtweise - Freiheit innerhalb eines Interdependenzmodells}

Die vorangegangene Aufarbeitung der Diskussion um die menschliche Freiheit sollte einen Überblick über verschiedene Perspektiven des Themas geben. Gerade im Bereich der Physik und der Biologie muss man die Argumente ernst nehmen, da hier auch die neuesten Erkenntnisse zum Thema DeterminismusIndeterminismus zu finden sind. Offensichtlich erscheint die Tatsache, dass menschliches Verhalten prinzipiell Restriktionen unterworfen ist. Ganz banale Aspekte wie die Schwerkraft oder genetische Vorgaben wie Augen- oder Haarfarbe führen zu einer Vielzahl von Einflussgrößen, die auf das gesamte menschliche Tun einwirken. Die angesprochenen wissenschaftlichen Erkenntnisse machen aber auch deutlich, dass offensichtlich Raum für freie Entscheidungen verbleibt. Freiheit im Sinne von Entscheidungsfreiheit und Wahl von Handlungsalternativen erscheint prinzipiell als möglich. Mit den folgenden Überlegungen soll nun ein soziologisches Modell von Freiheit entwickelt werden, das die zuvor angesprochenen Positionen berücksichtigt und weiterentwickelt. Die Kernfrage wird sein, ob man die Freiheit in Zusammenhang mit 
autonomen Einzelindividuen auch am richtigen Ort sucht. Das hier vorgeschlagene Modell von Freiheit soll im Weiteren als Basis für die Analyse der Veränderung von Freiheit in Deutschland dienen und Grundlage für die weiterführende Frage sein: Gibt es immer mehr Sicherheit zu Lasten der Freiheit?

\subsubsection{Interdependente menschliche Gesellschaft}

Die Diskussion um den freien Willen beruht auf der Vorstellung von einem autonomen Menschen, der als Individuum im Mittelpunkt der Überlegungen steht. Prinzipiell wird die Frage diskutiert, ob und inwieweit der Einzelne sich frei entscheiden kann oder nicht. Den Ursprung dieser gedanklichen Figur findet man beim Dualismus Descartes, bei dem der Geist und die Materie getrennt gedacht werden. Der so entstehende autonome Geist findet auch in der Soziologie im Rahmen des Voluntarismusansatzes - eng verwoben mit der Handlungstheorie - von TALCOTt Parsons ein passendes Pendant. Er geht davon aus, dass Menschen planvoll und unter Berücksichtigung von bekannten Daten in eine Situation gehen. Dabei werden die Daten selektiert und die ausgewählten Informationen nach bestimmten Kriterien mit Sinn erfüllt (vgl. AcKERMANN/PARSONS 1966: 70). Das sich anschließende Handeln ist nach PARSONS einerseits eng verknüpft mit der kulturellen und symbolischen Ebene (vgl. PARSONS 1966: 121). Gerade Symbole organisieren durch das Zuschreiben von Inhalten und Bedeutungen erst die erfolgreiche Kommunikation, sie wirken außerdem normativ regulierend, indem sie auch als Belohnung für bestimmte Bedürfnisse zur Verfügung stehen (vgl. PARSONS 1970: 386). Der enge Bezug zU WeBER (1921: 542), der bei seiner Definition von Handeln auf den subjektiv gemeinten Sinn verweist, wird an dieser Stelle der Theorie von PARSONS besonders sichtbar. Sinn und Intention der Handlung werden durch diese 
Zuschreibung erst von außen interpretierbar, wenn der subjektiv gemeinte Sinn verständlich wird. BRANDENBURG weist in diesem Kontext darauf hin, dass PARSONS davon ausgeht, dass Misserfolg oder Erfolg einer bestimmten Verhaltensweise über eine Art internes Belohnungssystem gesteuert werden und somit positiv sanktionierte Verhaltensweisen eher wiederholt werden (vgl. BRANDENBURG 1971: 143). Somit ließe sich dadurch der letztendliche, wirkliche Sinn der verschiedenen Handlungen feststellen. In enger Verbindung zu den Theorien PARsons steht Alfred SChüTz, der in verschiedenen Veröffentlichungen die Gedanken PARSONS aufgriff und reflektierte. Er verweist bei seiner Betrachtung des PARSONS'SCHEN Voluntarismus darauf hin, dass für das rationale planvolle Handeln das vernünftige Wissen bezüglich der eigenen Situation und ein Abwägen von Zweck und Mitteleinsatz bedeutsam seien (vgl. ScHüTZ 1977: 31). Die Positionen des Voluntarismus von PARSONS blieben nicht ohne Widerspruch. NORBERT ELIAS nimmt in der Einleitung zu seiner Zivilisationstheorie zum autonomen Einzelmenschen ausführlich Stellung:

\footnotetext{
„Die Vorstellung von den absolut unabhängig voneinander entscheidenden, agierenden und 'existierenden Einzelmenschen' ist ein Kunstprodukt, das für eine bestimmte Stufe in der Entwicklung ihrer Selbsterfahrung charakteristisch ist. Es beruht zum Teil auf einer Verwechslung von Ideal und Tatsache, zum Teil auf einer Verdinglichung der individuellen Selbstkontrollapparaturen und der Absperrung individueller Affektimpulse von der motorischen Apparatur, von der unmittelbaren Steuerung der Körperbewegung, von Handlungen« (ELIAS 1969: 65).
}

Die Kritik von EliAs bezieht sich ausdrücklich auf MAX WEBER und TALCOTt PARSOns (vgl. Elias 1969: 50), inhaltlich verweigert er sich dem vorherrschenden Dualismus von abgeschlossenen Einzelmenschen und einer davon isoliert betrachteten Gesellschaft (vgl. ELIAS 1969: 51). Als Alternative zeichnet ELIAS ein Menschenbild, das »absolute und totale Autonomie» (ELIAS 
1969: 67) nicht kennt, sondern diese dem Menschen nur graduell zubilligt. Die verschiedenen Abhängigkeiten der Menschen untereinander bezeichnet ELIAS als »Interdependenzen« (ELIAS 1969: 67). Sein Menschenbild geht konsequenterweise von Menschengruppen aus, Individuen ohne Gesellschaft und vice versa sind für inn keine angemessene Vorstellung von Gesellschaft. Nur in der Pluralität von Beziehungsgeflechten sind Menschen vorstellbar. ELIAS bezeichnet diese Beziehungsgeflechte als Figurationen (ELIAS 1969: 69). Für die Soziologie ElIAS ist in diesem Zusammenhang individuelle Freiheit als axiomatische Annahme kein notwendiges Element der soziologischen Theorie. Im Gegensatz etwa zum Rational-Choice-Ansatz ${ }^{1}$ ist es für ELIAS Theorie nicht entscheidend, ob Menschen bewusst und frei handeln, sondern nach welchen Gesetzmäßigkeiten Handeln sich vollzieht, unabhängig von Willensund Entscheidungsfreiheit. Im Kontext dieser wissenschaftlichen Analyse ergibt sich durch die Gedankengänge ELIAS eine neue Perspektive. Freiheit ist somit nicht primär eine Frage, die autonome Individuen betrifft, sondern muss auf einer übergeordneten Ebene gesucht werden. Dem steht die Alltagserfahrung der Menschen gegenüber, denen im Alltag bewusst zu sein scheint, freie Entscheidungen treffen zu können. Aus Alternativen wird immer wieder ausgewählt und die entstehenden neuen Situationen ergeben wiederum neue

1 Vgl. umfassend bei SCHMIDT 1998 und einleitend bei DIEKMANN/Voss (2004): Der Rational-Choice-Ansatz geht von bewusstem Handeln der Beteiligten nach bestimmten Annahmen aus. Die Handelnden greifen auf knappe Ressourcen zu, die von bestimmten Rahmenbedingungen abhängen. Gleichzeitig ist es wichtig, dass mindestens zwei Handlungsalternativen zur Verfügung stehen, die letztlich zu einer optimalen individuellen Zielerreichung führen sollen. Die Spieltheorie fungiert in diesem Zusammenhang als wichtiges Instrument zur Analyse, Interpretation und Prognose reziproker sozialer Interaktionen. 
Entscheidungssituationen, deren Ausgang erneut neu bestimmt werden kann. Trotzdem ist den meisten Menschen auch bewusst, dass es begrenzende Faktoren gibt: Physikalische, biologische und natürlich auch gesellschaftliche Rahmenbedingungen sind von entscheidender Bedeutung für das Handeln der Menschen und werden auch alltäglich ${ }^{1}$ wahrgenommen.

Die soziologische Analyse dieser Arbeit möchte die individuelle mit der übergeordneten sozialen Ebene verbinden. Das Hauptaugenmerk liegt auf den gesellschaftlichen Rahmenbedingungen. Dazu zählen beispielsweise verschiedene Kommunikationsformen, der Zugang und das Benutzen von Wissen, Rollenerwartungen, Normen in verschiedenen Kodifizierungsgraden und auch Formen der sozialen Kontrolle. Anhand der Ausgestaltung dieser unterschiedlichen Konditionen können verschiedene Aussagen über die Qualität von Freiheit in einer Gesellschaft getroffen werden.

Andererseits ist von Bedeutung, welche Auswirkungen diese Freiheit im Umkehrschluss auch auf die alltägliche Wahrnehmung des Einzelnen hat und wie eine Veränderung der Rahmenbedingungen tatsächliche Konsequenzen für das alltägliche Leben hat. Die folgenden Ausführungen beruhen auf ELIAS Vorstellung von Menschengeflechten, deren Mitglieder untereinander interdependent verbunden sind. Es soll versucht werden, innerhalb dieses Geflechts Freiheit zu positionieren. Die Interdependenz der Menschen kann modellhaft wie folgt (Abbildung 2) dargestellt werden. Eine Vielzahl von

1 Beispiele aus den drei Bereichen gibt es viele: Masse, Dichte und Trägheit sind Beispiele für den Bereich Physik; Geschlecht, Körpergröße, Körperkraft und ganz allgemein das Leistungsvermögen sind biologische Begrenzungsfaktoren; Einkommen, Bildung und die Zugehörigkeit zu bestimmten sozialen Schichten und Milieus wirken analog; 

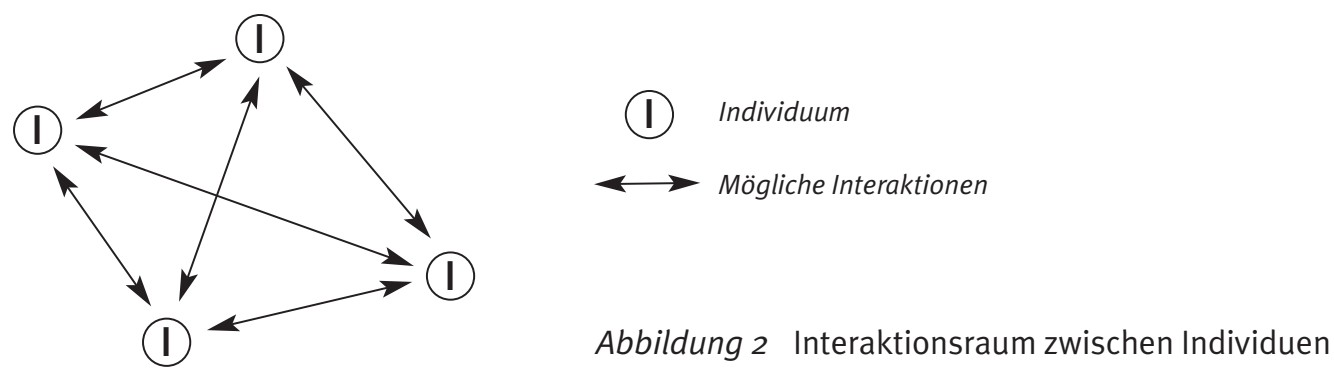

Individuen, die untereinander durch verschiedene Interaktionsformen verbunden sind, bildet interagierend ein Beziehungsgeflecht. Die Verbindungslinien zwischen den eingezeichneten Individuen stehen für mögliche Bündel von Interaktionen, die zwischen den einzelnen Partnern möglich sind. Erweitert werden kann das oben genannte gitterartige Modell, in dem man dreidimensionale Verbindungen von teilweise weit voneinander distanzierten Partnern (ähnlich dem menschlichen Gehirn mit seinen Neuronen) mit berücksichtigt. Zwischen den einzelnen Individuen einer Gesellschaft ergibt sich auf diese Weise ein gigantischer Raum an Interaktionsmöglichkeiten, die aber nicht alle getätigt werden müssen oder auch nur getätigt werden können. Die einfachste denkbare Interaktion innerhalb dieses Modells ist eine simple Face-to-faceSituation zwischen zwei Menschen, in der ein kommunikativer Austausch durch Mimik, Gestik und Sprache stattfinden kann. Nach Berger und LuckMANN ist diese Form einer »vis-à-vis-Situation« (BERGER/LUCKMANN 1980: 31) der »Prototyp aller gesellschaftlichen Interaktion« (ebd.)

Die Interaktion zwischen den Beteiligten beruht auf einem gemeinsamen Code, dessen Kenntnis für die Beteiligten enorm wichtig ist, da nur dann Bedeutungszuschreibungen der einzelnen Aktionen die Interaktion mit Sinn erfüllen. Das Interagieren und Interpretieren der Aktionen fassen BERGER und 
LUCKMANN als Prozess auf, dessen einzelne Bestandteile durch »Reziprozität« (ebd.) gekennzeichnet sind: Auf jede Aktion erfolgt in der Regel eine darauf aufbauende Reaktion, bis die Interaktion letztlich abbricht. Beide Teilnehmer sind bei diesem Prozess mehr oder weniger gleichberechtigte Partner, zumindest haben beide ähnliche Chancen auf Partizipation an dem gemeinsamen Austausch. Absolute Symmetrie ist allerdings keine Voraussetzung für Interaktionen nach diesem Modell. Bei einer enormen Anzahl an Kommunikationsformen, etwa über einen Fernsehbericht, eine Hörfunksendung, Zeitungsartikel oder auch bei einer Universitätsvorlesung bestehen nur eingeschränkte Möglichkeiten zu antworten und damit aktiv zu partizipieren. Die Asymmetrie bezüglich der Kommunikation muss sich dabei nicht nur auf die Redefähigkeit beschränken, sondern kann auch andere Ausformungen wie etwa Machtunterschiede haben. Bezieht sich die Asymmetrie in der Interaktion auf den Zugang zu bestimmten Ressourcen, die als »wertvolle Güter« (HRADIL 1999: 26) knapp und auch wünschenswert für die Menschen sind, kann man auch im Rahmen des Interdependenzmodells Grundlagen für Strukturen sozialer Ungleichheit entdecken.

Die zuvor schon festgestellte Analogie zum menschlichen Gehirn greift auch bei weitergehenden Betrachtungen. Obwohl prinzipiell alle gesunden Menschen über einen gleichartigen Gehirnaufbau mit Kleinhirn, Großhirn, Stammhirn und allen anderen Komponenten verfügen, unterscheiden sich die neuronalen Verbindungen bei jedem Einzelnen. Besonders Erlebnisse in der Kindheit sorgen im Gehirn für unterschiedliche Anordnungen. Gleiches gilt auch für die Interaktionen im Modell: Jeder Mensch hat einen grundsätzlich ähnlichen Zugang zu den Interaktionsmöglichkeiten, allerdings unterscheiden sie sich auch durch die jeweiligen individuellen Lebenssituationen, die sich 
ihrerseits aber dynamisch weiterentwickeln und verändern können. Zur Illustration kann man sich etwa einen gehbehinderten Menschen vorstellen, der eben durch seine speziellen Voraussetzungen von bestimmten Interaktionen, die sich etwa im Rahmen von bewegungsintensiven Aktivitäten ergeben, ausgenommen bleibt. Viele Lebensbereiche sind für diese Menschen aufgrund ihrer Einschränkungen nicht erreichbar, allerdings verändern sich auch manchmal über längere Zeiträume hinweg die gesellschaftlichen Rahmenbedingungen. Um beim Beispiel der Gehbehinderung zu bleiben: Immer mehr öffentliche Räume werden barrierefrei umgebaut und damit zugänglich. Ein weiterer wichtiger Aspekt ist die Interaktionshäufigkeit und die Interaktionsintensität, die abhängig von den Partnern schwanken kann. So haben wir im Normalfall zu Familienmitgliedern wesentlich öfter und auch intensiver Kontakt als zu Fremden. Vertraute Menschen werden beispielsweise mit liebevollen Gesten, Arbeitskollegen oder flüchtige Bekannte per Handschlag begrüßt. In diesem Sinne kann man die verschiedenen Interaktionen mit Hilfe der Kategorie Distanz ordnen (vgl. u. a LUTHE 1985), die nicht nur rein räumlich zu verstehen ist:

\footnotetext{
»Um jeden Menschen liegt eine ideelle Sphäre (.), nach verschiedenen Richtungen und verschiedenen Personen gegenüber freilich ungleich groß, in die man nicht eindringen kann, ohne den Persönlichkeitswert des Individuums zu zerstören. Einen solchen Bezirk legt die Ehre um den Menschen. (...) Der Radius jener Sphäre sozusagen bezeichnet die Distanz, deren Überschreitung durch eine fremde Persönlichkeit die Ehre kränkt» (SIMMEL 1968: 265).
}

Die Sphäre, die SIMMEL als die engste und wichtigste bezeichnet und die den materiellen Besitz umfasst - zu letzterem zählt SIMMEL auch den Körper des Menschen - leidet erheblich durch ein willentliches Eindringen von Außen:

»Wie das materielle Eigentum gleichsam eine Ausdehnung des Ichs ist - Besitz ist eben, was dem Willen des Besitzers gehorcht, wie, in nur gradueller Unterschiedenheit, der 
Leib, der unser erster Besitz ist - und wie deshalb jeder Eingriff in den Besitzstand als eine Vergewaltigung der Persönlichkeit empfunden wird, so gibt es ein geistiges Privateigentum, dessen Vergewaltigung eine Lädierung des Ichs in seinem Zentrum bewirkt« (SIMMEL 1968: 266).

Es sind aber nicht nur die unterschiedlich großen Distanzen, welche die Interaktionen der Menschen ordnen und strukturieren. Die Interaktionen selbst werden durch drei verschiedene Aspekte beeinflusst und determiniert: durch Motivation, Situation und durch die Verhaltenserwartungen potentieller Interaktionspartner (vgl. LAMNEK 1979: 13). Normen strukturieren den Raum möglicher Interaktionen, in dem sie einerseits entsprechende Sanktionen der Interaktionspartner, wie etwa Rache, Strafe oder Belohnungen, für das Verhalten quasi versprechen. Durch die subjektive Perzeption der Sanktionswahrscheinlichkeit wirken solche Normen dann verhaltenseinschränkend. Andererseits bestimmen die gleichen Normen auch die Verhaltenserwartungen der Aktionspartner und wirken somit nochmals auf die Handlung ein. Von der Norm abweichendes Verhalten ist im Rahmen der verschiedenen Interaktionen ebenfalls möglich, allerdings wirkt diesen Verhaltensweisen die soziale Kontrolle entgegen. Darunter sollen hier alle »Prozesse und Mechanismen zur Verhinderung oder Einschränkung (.) abweichenden Verhaltens in einer Gesellschaft« (LAMNEK 1979: 304) verstanden werden. Die Sanktionen machen genau diese Kontrolle aus, die zusätzlich durch Sozialisations- und Enkulturationsprozesse verinnerlicht werden und damit der im Inneren ablaufenden Eigen-Sanktionierung (Schuldgefühle, gutes Gewissen) überantwortet werden (vgl. ebd.: 305).

Ähnlich wie das Recht eine Unterscheidung zwischen natürlichen und juristischen Personen trifft, darf nicht unterschlagen werden, dass neben den interdependent verbundenen Menschen auch Gruppierungen und Organisa- 
tionen Teile des Zusammenwirkens sind. Das kann zum Beispiel ein Unternehmen sein, ein Verband, eine Institution und selbstredend auch der Staat, etwa in Form seiner ausführenden Organe (Polizei, Finanzbehörden, Amt für Denkmalschutz, etc.).

Zum Abschluss dieses Interaktionsmodells muss man wieder zurück zur zentralen Frage kommen: Wie lässt sich Freiheit begreifen und wie kann das Modell dazu beitragen, sie exakt zu bestimmen?

\subsubsection{Objektive und subjektive Freiheit innerhalb interdependenter Gesellschaften: Kontrolle als entscheidender Faktor}

Um aus der soziologischen Perspektive heraus Freiheit in interdependenten Gesellschaften korrekt einordnen zu können, soll hier zunächst dargestellt werden, auf welchen soziologischen Überlegungen, die Unterscheidung in objektive und subjektive Freiheit beruht. Die Grundlagen für diese Differenzierung liefern Überlegungen des Soziologen MERTON und seine Theorie der self-fullfilling prophecy, die dieser Mitte des 20 Jahrhunderts erstmalig präsentierte. Merton stellte 1957 in einem gleichnamigen Aufsatz erstmalig das Konzept vor, das im Kern auf dem so genannten Thomas-Theorem, das nach W.I. Thomas und seiner Frau Dorothy Thomas (vgl. u.a. Merton 1995: 380) benannt ist. Es besteht aus dem prägnanten Satz: »If men define situations as real, they are real in their consequences« (THомAS/THомAS 1928: 572). Merton greift dieses Theorem auf leitet daraus ab, dass Menschen ihr Verhalten nicht nach den »objektiven Gegebenheiten einer Situation« (MERTON 1965: 145) ausrichten, sondern nach der Bedeutung der Situation für sie. Er illustriert dies anhand eines geradezu berühmten und immer noch aktuellen 
Beispiels des Scheiterns eines Bankinstituts; er bezieht sich hier auf die Last National Bank, über die zunächst Pleitegerüchte kursieren und die dann, als Kunden zur Vermeidung eines Verlustes ihr Guthaben abziehen, tatsächlich Konkurs anmelden musste, obwohl rein objektiv betrachtet die Bank zuvor keinerlei Liquiditätsprobleme hatte (vgl. SCHNEPPER 2004: 25). Die subjektive Perspektive der Akteure schlägt sich auf die objektiven Gegebenheiten nieder. Somit wird die Akteurspersepktive hier zum bestimmenden Element (ebd.). MERTON spricht in diesem Zusammenhang von einer so genannten self-fullfilling prophecy, die von einer ursprünglich falschen Annahme ausgeht, die neue Verhaltensweisen auslöst, die ihrerseits wieder die falschen Ausgangsbedingungen »wahr« werden lässt (vgl. MERTON 1965: 146) die falsche Prophezeiung hat sich erfüllt und ist soziale Realität geworden. MERTON zeigt in seinen weiteren Ausführungen an gleicher Stelle, wie das Prinzip der self-fullfilling prophecy unter anderem zur Verfestigung von Vorurteilen führen kann, die sich durch die angesprochenen Mechanismen in der jeweiligen Rückschau als harte Fakten tarnen (ebd. 147), obwohl dies realiter gerade nicht der Fall ist. Das Gegenstück zur self-fullfilling prophecy ist bei MERTON die sogenannte »suicidal prophecy«, die das menschliche Verhalten so verändert, dass die getroffene Voraussage nicht Wirklichkeit wird (ebd. 161). In beiden Fällen bekommen die getroffenen Voraussagen über die gesellschaftliche Realität eine Eigendynamik, die durch selektive Wahrnehmung und Rezeption und damit durch subjektive Interpretation gekennzeichnet ist und sich nicht mehr an objektiven Gegebenheiten orientiert (vgl. SCHNEPPER 2004: 24).

Freiheit lässt sich vor oben skizzierten Hintergrund analysieren und einordnen, wobei man analog zwischen objektiver und subjektiver Freiheit unter- 
scheiden kann. Unter objektiver Freiheit soll hier Freiheit in einem »messbaren«, objektivierbaren Sinn verstanden werden; es gilt auf objektiver Ebene einzuschätzen, wie sie sich verändert. In enger Verbindung damit steht die subjektive Freiheit, die auf der Wahrnehmung von Freiheitsbedingungen der Individuen einer Gesellschaft beruht. Gemäß der self-fullfilling prophecy Mertons, kommt der subjektiven Perpektive einige Bedeutung zu, da die subjektive Interpretation von Freiheit im Zirkelschluss auf die gesellschaftlichen Ausgangsbedingungen Einfluss nimmt.

Es soll hier im Weiteren versucht werden, den Begriff Freiheit auf mehreren Ebenen in das im letzen Kapitel vorgestellten Modell der interdependenten Gesellschaft einzuordnen. Ein wichtiger Aspekt, den es zu berücksichtigen gilt, ist, dass das Augenmerk auf der Entwicklung von Freiheit liegt, vor allem in besonderem Bezug zur Sicherheit. Somit liegt das letztendliche Ziel darin, Freiheit nicht nur statisch zu fixieren, sondern sie prozessual zu interpretieren, um mögliche Veränderungen erkennbar zu machen. In einem ersten Schritt könnte man Freiheit im Sinne reiner Quantität auffassen. Je größer die Anzahl möglicher Interaktionspartner und je größer die Anzahl möglicher Interaktionen, umso größer die Freiheit der Gesamtgesellschaft. Konzentriert man den Begriff Freiheit auf die Größe des Interaktionsraumes im Sinne von Alternativentscheidungen, so passt die getroffene Aussage in das Modell. Zu berücksichtigen bleibt aber die Tatsache, dass die einzelnen Akteure eben interdependent verbunden sind und somit im Einzelfalle zu prüfen ist, wie sich eine Veränderung auf die anderen Akteure auswirkt. Dies ist aufgrund der großen Zahl an Variationen nur tendenziell möglich. Zusätzlich wird die korrekte Einschätzung einer Veränderung auch dadurch erschwert, dass die Wirkungen eines Ereignisses für die Individuen einer Gesellschaft nicht unmit- 
telbar auftreten, sondern über mehrere Interaktionssequenzen hinweg wirken. Als Beispiel für ein solches Ereignis soll hier die hypothetische Legalisierung von Marihuana genannt werden. Die unmittelbare Konsequenz der Freigabe wäre die Möglichkeit des strafrechtlich nicht sanktionierten Konsums, also für einen Teil der Bevölkerung eine quantitative Erweiterung des Interaktionsrahmens, wobei hier nicht zwischen positiven und negativen Folgen der Legalisierung unterschieden werden soll. Auch andere Akteure wie die Polizei, die Justiz, die Politik, das Gesundheitssystem oder Drogenberatungsstellen wären betroffen und würden allein durch den Umstand, dass der Konsum legalisiert wurde, Veränderungen erfahren. Beispielsweise würden bei der Polizei und im Justizwesen finanzielle und personelle Ressourcen frei, die möglicherweise anderen Bereichen der Polizeiarbeit zu gute kämen, Drogenberatungsstellen müssten neue Positionen und Handlungsweisen bezüglich der Legalität des Konsums beziehen. Die Folgen eines Ereignisses sind zudem in der Regel ambivalent, wie das Beispiel Umweltschutz illustrieren kann. Umweltschutzmaßnahmen schränken den Einzelnen und auch Unternehmen ein, Regeln zur Entsorgung von Abfall begrenzen den Handlungsspielraum. Statt Müll beliebig abladen zu können, muss stattdessen recycelt werden. Für die Beteiligten reduzieren sich die Handlungsoptionen in dem Sinne, dass künftig bestimmte Handlungen sanktioniert werden, etwa durch entsprechende Bußgelder. Gleichzeitig erhöhen sich durch die Bewahrung der Natur auch die Spielräume der Beteiligten, etwa für die Tourismusverbände, für den Einzelnen, der die intakte Natur genießen kann oder auch für Firmen, die ihr ökologisches Engagement in Form von Werbemaßnahmen und zur Imagesteigerung nutzen. In der Gesamtschau ist die skizzierte quantitative Interpretation von potenziellen Alternativen nicht alleine aussagekräf- 
tig. Aus dem Blickwinkel der einzelnen Menschen lässt sich Freiheit ergänzend mit dem Begriff der Kontrolle besser fassen. Kontrolle ist hier nicht verstanden im Sinne von sozialer Kontrolle als Überwachung oder dem Herstellen von Konformität. Im Gegensatz dazu soll als Kontrolle der Grad an Einflussmöglichkeit bezeichnet werden, der dem Einzelnen auf die ablaufenden Interaktionen gegeben ist. Da wichtig ist, in welchem Umfang der Einzelne diese Kontrolle ausüben kann, soll hier im Weiteren von Eigenkontrolle in Abgrenzung zur Fremdkontrolle die Rede sein. Als Fremdkontrolle soll der Anteil an Fremdeinfluss auf existierende Interaktionsformen bezeichnet werden. Blickt man zurück auf die Distanz als wichtigem Element innerhalb des Interdependenzmodells, so bleibt festzuhalten, dass die Formen der Kontrolle umso wichtiger werden, je unmittelbarer sie die innerste Sphäre des Individuums betreffen. Mit Letzterer ist damit in räumlicher Sichtweise die unmittelbare Lebensumgebung von den eigenen vier Wänden und in sozialer Hinsicht sind nahe stehende Personen gemeint. Bei Interaktionen mit Individuen, die im Beziehungsgeflecht weiter entfernt stehen, ist der Anteil an Eigenkontrollmöglichkeit von vorne herein geringer. Ein Blick auf die Wohnsituation kann dies veranschaulichen. Die eigene Wohnung oder das eigene Haus sind der Kernbereich des Privaten. In der Regel ist die Kontrolle über dieses Reich groß. Die Bewohner haben es in der Regel gebaut oder zumindest individuell gestaltet, wissen, wo die Möbel stehen und welches Eigentum sich wo befindet. Abhängig von der Bauweise können sich die Bewohner vor Interaktionen, in dem Falle beispielsweise vor Außenkontakten, verbergen, indem sie einfach das Telefon ignorieren, die Vorhänge als Blickschutz zuziehen und die Tür bei einem Klingeln nicht öffnen. Verlässt man aber die eigenen vier Wände, nimmt das Maß an Eigenkontrolle kontinu- 
ierlich ab. Bereits der Garten ist eine „Pufferzone“ (LAMNEK 2003: 59) zwischen Privatheit und Öffentlichkeit, wo das gegebene Maß an Kontrolle graduell abnimmt. Schon die visuellen Eindrücke des Hauses und des Grundstückes lassen sich nicht mehr im vollen Umfang kontrollieren. Geht man weiter vom eigenen Zuhause weg, verstärkt sich der Effekt des zunehmenden Verlustes der Eigenkontrolle. Wir können Begegnungen mit anderen Menschen nur noch schwer vermeiden, noch können wir die Inhalte der Interaktion kontrollieren: Wir wissen nicht, ob der Fremde, der auf uns zutritt, nach dem Weg fragen möchte oder ob er ein anderes Anliegen hat.

Festzuhalten in diesem Sinne bleibt, dass Veränderungen der Freiheit für die Menschen einer Gesellschaft sich auf unterschiedlichen Distanzen abspielen können. Sowohl Kontrollverlust durch Eingriffe in die Privatsphäre als auch auf entferntere Sphären sind denkbar und können auch gemeinsam auftreten. Die Kapitel 3 und 4 werden Fälle beider Kategorien intensiv diskutieren.

\subsubsection{Wissen und andere sekundäre Bedingungen für Freiheit}

Die Kontrolle des Einzelnen bezüglich der Formen und des Umfangs der Interaktion ist der wichtigste Indikator für die Beurteilung der Freiheit einer Gesellschaft. Das Ausmaß der Eigen- beziehungsweise der Fremdbestimmung muss aber nicht zwangsweise für alle Gesellschaftsbereiche in gleichem Umfang gelten. In vielen Bereichen ist die Eigenkontrolle gering und die Einflüsse von Außen bestimmen weitgehend das gesamte Leben. Gofrmanns totale Institutionen (vgl. GoFFMANN 1972) lassen sich solchen fremdbestimmten Bereichen zuordnen. Er unterscheidet dabei fünf verschiedene Gruppen (ebd.: 16): 
- Anstalten, die der Fürsorge dienen, wie Altenheime und Waisenhäuser;

- Anstalten, die der Fürsorge für Menschen dienen, die sich nicht selbst helfen können (Krankenhäuser);

- Anstalten zum Schutz der Allgemeinheit vor Gefahren (Gefängnisse);

- Institutionen, bei denen der Zweck die totale Organisationsform zu bedingen scheint (Schiffe, Internate, Arbeitslager, etc.);

- Zufluchtsorte wie Klöster und andere mönchische Wohngemeinschaften.

Das zentrale Merkmal von solchen totalen Institutionen ist nach GofrMANN der Umstand, dass eigentlich getrennte Lebensbereiche miteinander kombiniert werden:

\begin{abstract}
»1. Alle Angelegenheiten des Lebens finden an ein und derselben Stelle, unter ein und derselben Autorität statt. 2. Die Mitglieder der Institution führen alle Phasen ihrer täglichen Arbeit in unmittelbarer Gesellschaft einer großen Gruppe von Schicksalsgenossen aus, wobei allen die gleiche Behandlung zuteil wird und alle die gleiche Tätigkeit gemeinsam verrichten müssen. 3. Alle Phasen des Arbeitstages sind exakt geplant, eine geht zu einem vorher bestimmten Zeitpunkt in die nächste über, und die ganze Folge der Tätigkeiten wird von oben durch ein System expliziter formaler Regeln und durch einen Stab von Funktionären vorgeschrieben 4. Die verschiedenen erzwungenen Tätigkeiten werden in einem einzigen rationalen Plan vereinigt, der angeblich dazu dient, die offiziellen Ziele der Institution zu erreichen« (ebd.: 17).
\end{abstract}

Die Fremdkontrolle ist in solchen Institutionen sehr hoch, obwohl Freiräume verbleiben, deren Ausnützung oft im eklatanten Gegensatz zu den erklärten Zielen der jeweiligen totalen Institution steht. Selbstmorde oder auch Morde an Mitgefangenen (vgl. HolzhaIder 2007) in hochgradig fremdkontrollierten Umgebungen wie Gefängnissen sind ein Beispiel dafür.

Ein Problem der Eigenkontrolle ist die Tatsache, dass sie unmöglich von jedem Individuum alleine gemeistert werden kann. In der Praxis lässt sich eine 
Vielzahl an sekundären Mechanismen feststellen, die dem Einzelnen die Eigenkontrolle erleichtern und welche die Kontrollausübung weg vom Individuum hin zu größeren sozialen Gebilden verlagert. Die wichtigste dieser Hilfen ist das Wissen, das unbedingte Voraussetzung für Kontrolle und damit auch für Freiheit ist. Der Einfluss des Wissens lässt sich dabei differenziert betrachten. Wissen im Sinne von bloßer Quantität, bzw. einem Mehr an Information, eröffnet durch die Kumulation von Informationen neue Handlungsalternativen. In der Entwicklung der meisten Menschen geht dieser massive Wissensfortschritt mit dem Erwerb der Schriftsprache einher. BACHL bezeichnet in diesem Zusammenhang das Erlernen des Lesens als einen »Exodus aus der bekannten Welt« (BACHL 1989: 15). Dem Erstleser erschließen sich nach und nach immer größere Teile des geschriebenen Wortes, er erhält das Potenzial, auf das gesamte kulturelle Wissen seiner Gesellschaft zugreifen zu können und ist nicht mehr auf mündliche Kommunikation als alleiniges Mittel zum Erwerb von Wissen angewiesen. Das Wissen selbst wird durch seine Niederlegung in Schriftform auch zeitunabhängig, es bleibt im Gegensatz zur mündlichen Überlieferung fixiert und kann wiederholt und auch in unveränderter Form von verschiedenen Rezipienten abgerufen werden. Für das Individuum hat das Erlernen des Lesens weitreichende Auswirkungen für die geistige Entwicklung. Vor allem im Bereich der Phantasie sorgen Bücher für ein Training der Vorstellungskraft, bei dem die verschiedenen Symbole des Textes mit Sinn und Aussage gefüllt werden müssen. Der Leser kann verschiedene Situationen im Kopf durchspielen und so schriftlich fixierte Gedankengänge anderer nachvollziehen. So wird das Abstraktionsvermögen geschult und durch das Erlernen von Schreibkompetenzen entwickelt sich das Vermögen, auch die eigenen Gedanken anderen schriftlich zur Verfügung stellen zu können. Diese 
Fertigkeit wird im Rahmen der gesamten Schulausbildung in unterschiedlicher Intensität trainiert. Wissenssoziologisch ${ }^{1}$ betrachtet hat Wissen aber noch weitergehende Bedeutung für den Einzelnen und die Gesellschaft. Die verschiedenen Formen alltäglichen Wissens regulieren nach BERGER und LUCKMANN das Verhalten in der Alltagswelt (BERGER/LUCKMANN 1980: 21), auf der die »subjektiv sinnhafte(.) Lebensführung von jedermann« (ebd.: 219) beruht. Im Umkehrschluss verdankt die Alltagswelt aber auch »jedermanns Gedanken und Taten ihr Vorhandensein und ihren Bestand« (ebd.: 21f.). Sie und das damit verbundene Wissen sind um die einzelnen Individuen herum angeordnet, verschiedene Distanzen strukturieren sie hinsichtlich der Relevanz. Wichtig ist, dass Welt und Wissen mit anderen Menschen geteilt und damit intersubjektiv werden (ebd.: 25): Im Zusammenspiel mit den anderen Menschen konstruiert sich die gesellschaftliche Wirklichkeit. Dieser kontinuierliche Prozess läuft nicht immer bewusst ab. Auf die Postionen von Foucault und Bourdieu, die Wissen im Kontext von Macht, Erzeugung und Verteilung bzw. im Kontext der Verfestigung zu Habitusformen einschätzen, soll hier vorerst verzichtet werden. Im Kontext von Sicherheit und Freiheit steht Wissen in dieser Arbeit eher pragmatisch im Mittelpunkt. Der FouCAULT'SCHE Kontext, der Macht «als integrierten Teil der gesamten Gesellschaft« (KNOBLOCH 2005: 213) und Wissen in enger Verbindung damit sieht, ist hier ebenfalls

1 Die Wissenssoziologie beschäftigt sich mit allem, was in einer Gesellschaft als Wissen gilt (BERGER/LUCKMANN 1980: 16). Dies gilt für die theoretischen Perspektiven von Intellektuellen und auch für das allägliche Wissen der »Normalverbraucher « (BERGER/LUCKMANN 1980: 21). Die Erweiterung der Wissenssoziologie um das Alltägliche ist der Verdienst von BERger und LuCKMANN, ihre Perspektive gilt für viele aktuell als das, was Wissenssoziologie eigentlich ausmacht (auch kritisch dazu vgl. MAASEN 1999: 26). 
sekundär, da Wissen in erster Linie als Voraussetzung für Eigenkontrolle und die damit verbundene Kommunikationskompetenz zu sehen ist, die für die Selektion und Interpretation der Informationen wichtig ist. Trotzdem hat das individuelle Wissen eine erhebliche Bedeutung bei der Partizipation an der Ausgestaltung der intersubjektiven Wirklichkeit. Wissen hat neben den oben genannten Aspekten auch eine unmittelbare praktische Relevanz bei der Ausübung von Eigenkontrolle. Nur wenn man eine Fremdbeeinflussung wahrnehmen kann und wenn man weiß, dass sie überhaupt existiert, kann man sich mit dieser überhaupt erst auseinander setzen und eventuell das individuelle Verhalten anpassen, so dass meine Freiheit gewahrt bleibt. Ein klassisches Beispiel ist die akustische Wohnraumüberwachung, von der später noch ausführlich die Rede sein wird. Das Abhören einer Kommunikation innerhalb der eigenen vier Wände ist der Kontrolle völlig entzogen, wenn man weder Wissen über die technischen Möglichkeiten noch Kenntnis von der tatsächlichen Umsetzung in seiner unmittelbaren Umgebung hat. Gegenmaßnahmen, welche die Freiheit in bestimmtem Umfang restaurieren könnten, können dadurch gar nicht erst erwogen werden. Das Wissen von diesen Dingen ermöglicht es aber, langfristig gegen bestimmte Elemente der Fremdbestimmung vorzugehen und die Freiheit eventuell wieder zurück erlangen zu können.

Das Beispiel macht aber auch klar, dass das Ringen um Eigenkontrolle nicht alleine geführt werden kann. Neben dem Wissen sind weitere Hilfen nötig, die man den Begriffen Kontrolle und Wissen unterordnen kann. Wissen benötigt Verbreitungswege, also Medien, die vielfältige Informationen liefern können, denn alles, „was wir über unsere Gesellschaft, ja über die Welt, in der wir leben, wissen, wissen wir durch die Massenmedien« (LuHMANN 1995: 9). Dieser Wissenstransfer funktioniert aber in der Regel nur in eine Richtung, 
weil eine unmittelbare Interaktion zwischen Sender und Empfänger verhindert (LUHMANN 1995: 6) und nur über Leserbriefe und Ähnliches nachträglich ermöglicht wird.

Vor allem die redaktionelle Freiheit in den Medien ist für diese Vielfalt wichtig, ebenso die Möglichkeit, als Einzelner ebenfalls an der Produktion von Wissen zu partizipieren. Die auf diese Weise anwachsende Informationsflut benötigt Strukturen, um verständlich zu werden. Neben technischen Aufbereitungsmöglichkeiten ist Bildungskompetenz - gemeint ist hier die Fertigkeit, Informationen aufzunehmen, sie in kulturelle und historische Kontexte zu stellen und sie damit sinnvoll zu machen - eine entscheidende Hilfe. In verwandter Weise ist auch das wissenschaftliche Denken im Sinne einer Wissensstrategie relevant.

Wichtige Voraussetzung dafür, dass Wissen Eigenkontrolle ermöglichen kann, sind sowohl Pressefreiheit als auch das Recht auf freie Meinungsäußerung. Verschiedene Freiheitsrechte und festgelegte, institutionalisierte Strukturen, wie diese Rechte auch durchgesetzt werden können, sind ebenfalls wichtige Hilfen bei der Kontrolle durch Individuen. Man mag in diesem Zusammenhang zu Recht die Frage aufwerfen, ob das dann nicht schon Fremdkontrolle durch andere Einrichtungen ist. Trotzdem soll hier beispielsweise das Ausschöpfen von Rechtswegen durchaus als Form der Eigenkontrolle gelten: Man muss den Weg nicht beschreiten, doch wenn man es denn tut, besteht die Chance, den Fremdeinfluss zurück zu drängen. Letztlich bekommt durch das Einfordern von Rechten, die schriftlich als Gesetze niedergelegt sind, das Ringen um Eigenkontrolle auch eine zeitliche Dimension. Wenn man auf der Straße zum Beispiel angegriffen und verletzt wird, so stellt dieses Ereignis einen Verlust der Eigenkontrolle dar: Man war nicht in der Lage, die eigene 
physische Integrität zu wahren. Durch eine entsprechende Anzeige mit einer sich anschließenden möglichen Verurteilung des Täters kann Kontrolle mit Hilfe anderer zurück erlangt werden. Beispielsweise würde eine Freiheitsstrafe für einen bestimmten Zeitraum den Täter am Durchführen weiterer Taten hindern und durch Resozialisierung - oder auch durch Abschreckung - künftige Straftaten verhindern.

In der Praxis sind die Menschen in erheblichem Maße auf übergeordnete Institutionen und Organisationen angewiesen. Sichere Verfahrenswege und einklagbare Rechte helfen beim (Zurück)Erlangen der Eigenkontrolle. Ändern sich die hier skizzierten Rahmenbedingungen einer Gesellschaft, dann kann sich auch der Grad an Freiheit massiv verändern. Auf der Basis des Interdependenzmodells und unter Berücksichtigung der besonderen Wirkmechanismen von Kontrolle, auf der Basis von Wissen, kann aussagekräftig beurteilt werden, wie sich die Freiheit innerhalb einer Gesellschaft verändert. Es gilt nun in ähnlicher Weise auch das Phänomen Sicherheit so zu erfassen, dass es möglich wird, die Entwicklung von Sicherheit und Freiheit in ihrem Zusammenhang analysieren zu können. Am Ende steht die Beantwortung der Frage, ob es in unserer Gesellschaft eine Entwicklung hin zu mehr Sicherheit auf Kosten der Freiheit gegeben hat oder eben nicht.

\subsection{Sicherheit}

Sicherheit wird in ähnlicher Art und Weise wie Freiheit häufig im alltäglichen Sprachgebrauch verwendet. Nicht immer sind die verschiedenen Bedeutungen, die dem Begriff Sicherheit zugeschrieben werden, übergreifend deckungsgleich. Im Rahmen dieser Untersuchung ist es deshalb ebenfalls 
wichtig, einen möglichst präzisen Zugang zu den Inhalten des Begriffs Sicherheit zu finden, um ihn der Freiheit angemessen gegenüber stellen zu können. Die folgenden Überlegungen werden verschiedene Bereiche von Sicherheit näher erörtern, um die Basis für die sich anschließende gemeinsame Betrachtung von Freiheit und Sicherheit und ihr besonderes Zusammenspiel bilden zu können.

\subsubsection{Sicherheit als Begriff}

Nähert man sich auf der sprachlichen Ebene dem Begriff Sicherheit, so kann man ihn auf das lateinische Securitas zurückführen. Securitas war ähnlich wie Pax (Frieden), Aeternitas (Ewigkeit), Roma (die Stadt Rom) oder Felicitas (Glück) eine anthropomorphe Personifizierung, die als gottähnliche Persönlichkeit verehrt wurde. Die Personifizierung zeigt, dass schon in der Antike Sicherheit für die Menschen außerordentlich bedeutsam war. Im Laufe der Zeit wurde Securitas in verschiedenen Sprachen in Form diverser Abwandlungen übernommen. So kennt beispielsweise das Englische die security, das Französische die sécurité und das Deutsche eben den Begriff Sicherheit. Bemerkenswerterweise steht der Ausdruck Securitas heute auch für den Namen einer international operierenden Firmengruppe für Sicherheitsdienstleistungen mit mehr als 250000 Mitarbeitern in 30 Ländern, von denen ca. 19000 an 70 Standorten in Deutschland tätig sind (securitas.de 2008). Grammatikalisch betrachtet kann man Sicherheit einem Lexemverband zuordnen, der sich um den Wortstamm sicher gruppiert. Eine enorme Begriffsvielfalt aus verschiedenen Bereichen wie beispielsweise sicher, sicherlich, Versicherung, Sicherheitstechnik, Sicherung, Informationssicherheit, Fahrzeugsicherheit, selbstsicher, Staatssicherheit, Sicherungshypothek, Sicherungsverwahrung, 
Sicherheitsschloss, Sicherungsmaßnahmen, Sicherungskopie, Sicherheitsdienst, Sicherheitspolitik, Sicherheitsbehälter, öffentliche Sicherheit oder Kindersicherung lässt sich dabei auflisten, natürlich ohne jemals Vollständigkeit gewährleisten zu können. Die Vielfalt der verwandten Begriffe verdeutlicht, dass Sicherheit ein recht umfassendes Phänomen darstellt, das mit vielen Bereichen des menschlichen Lebens in Verbindung steht. Eine verbindliche und umfassende Definition von Sicherheit fällt auf den ersten Blick zusätzlich schwer, da individuelle Erlebnisse die Vorstellung von Sicherheit bestimmen. MARCUSE bringt diese persönliche Komponente von Sicherheit auf den Punkt:

\footnotetext{
»Wer nie ein Erdbeben erlebt hat, wird nicht zur Sicherheit rechnen, daß die Erde unter seinen Füßen unbeweglich ist. Wer nie Atemstörungen hatte, wird nicht zur Sicherheit rechnen, daß der Verkehr zwischen Lunge und Luft ohne Schwierigkeiten vor sich geht. Wer nie ein Arbeitsloser gewesen ist, wird nicht zur Sicherheit rechnen, daß für den nächsten Tag Vorrat da ist. Und wer nicht ein Volk zum Nachbarn hat, das über einen herzufallen pflegt, wird nicht zur Sicherheit rechnen, daß eine chinesische Mauer oder eine Maginot-Linie die Grenzen schützt. Ein Jäger, auf einer einsamen Insel der einen Hasen geschossen und ein Felsloch gefunden hat, fühlt sich vielleicht sicher; und ein Millionär, in seinem New Yorker Palast, fühlt sich vielleicht unsicher, weil von einem russisch-japanischen Bündnis gemunkelt wird.« (MARCUSE 1981: 26)
}

Es mag dieser Vielfalt geschuldet sein, dass Sicherheit sich einer positiven Definition, welche festgelegte Aspekte beinhaltet, auf den ersten Blick entzieht. Stattdessen wird Sicherheit oftmals als ein "Zustand der Abwesenheit von Gefahrenbedrohung und des Freiseins von Angst umschrieben, der es dem einzelnen erlaubt, sich frei zu entfalten« (WIDMER 1995: 11f.). Daraus lassen sich die Begriffe Unsicherheit, Gefahr, Ungewissheit und Risiko als die Gegenpole zur Sicherheit ableiten, deren Abwesenheit erst Sicherheit möglich macht. Diese Begriffsbestimmung mag hier als Arbeitsdefinition vorerst 
genügen, allerdings soll der Begriff der Sicherheit später nach bestimmten Inhalten weiter ausdifferenziert werden. Es existieren diverse Ansätze, die sich mit dem Thema Sicherheit ausführlich beschäftigen, u. a im Bereich der Soziologie durch MURCK (1980) und vor allem auch durch KAUFMANN (1970). Die folgende Klassifikation geht von beiden soziologischen Ansätzen aus, setzt aber vor allem bei der politischen, technischen und rechtlichen Dimension Schwerpunkte:

- Politische Sicherheit (bestehend aus innerer und äußerer Sicherheit);

- Technische Sicherheit;

- Wirtschaftliche Sicherheit;

- Soziale Sicherheit;

- Rechtssicherheit.

Politische Sicherheit umfasst sowohl die öffentliche Sicherheit, die sich auf das Innere eines Staates bezieht, als auch die nationale und internationale Sicherheit, die sich nach Außen orientiert. Nach KROLLMANN (1972: 17) umfasst die öffentliche Sicherheit die »Unversehrtheit von Leben, Gesundheit, Ehre, Freiheit und Vermögen der Bürger, sowie der Rechtsordnung und der Einrichtungen des Staates«. Im Mittelpunkt stehen hier sowohl der einzelne Bürger als auch der Staat mit seiner Rechtsordnung und wichtigen politischen Institutionen. Auch die Unversehrtheit der Freiheit gehört nach dieser Definition zu den zu Gütern, die eingebettet in den Kontext der rechtlichen und institutionellen Strukturen abzusichern sind. Offen bleibt bei dieser Definition von öffentlicher Sicherheit, wer diese ermöglichen und garantieren soll.

In enger Verbindung mit dem Begriff der öffentlichen Sicherheit steht die so genannte innere Sicherheit, die den Schutz einer Gesellschaft vor Krimina- 
lität und verwandten Bedrohungen bezeichnet, die sich aus der Gesellschaft selbst entwickeln oder teilweise, etwa im Bereich von grenzüberschreitender Kriminalität, auch von außen kommen kann. Das Bundesinnenministerium definiert Innere Sicherheit in diesem Sinne recht knapp; sie umfasse ein weites Themenspektrum »von der Kriminalitäts-, Terrorismus- und Extremismusbekämpfung über Verfassungsschutz, Geheim- und Sabotageschutz sowie Bevölkerungsschutz im Krisenfall bis zu Bundespolizei, Waffenrecht oder Sicherheit in der Informationstechnik« (Bundesministerium des Innern 2006, zitiert nach Feltes 2006: 105). Feltes bemängelt an dieser Definition die Konzentration auf Repression und Kontrolle und verweist auf LANGE, der eine erheblich erweiterte Definition von innerer Sicherheit gibt. Dieser definiert in seiner Politikfeldanalyse »Innere Sicherheit« wie folgt: »Das Politikfeld Innere Sicherheit umfaßt alle staatlichen Institutionen und Einrichtungen sowie staatlich beauftragten Organisationen, die durch Verfassung und Organe der demokratischen Willensbildung legitimiert sind, das öffentliche Gewaltmonopol im Rahmen kodifizierter Regeln exekutiv auch unter Anwendung von unmittelbaren Zwang auszuüben.« (vgl. LANGE 1999: 109). Er verweist an gleicher Stelle ebenfalls darauf, dass neben den Einrichtungen der Exekutive (Staatsanwaltschaft und Polizei) auch weitere Akteure wie das »Innenministerien, parlamentarische Institutionen, Parteien, Verbände, föderale Verhandlungsgremien ebenso wie gesellschaftliche Gruppen und Medien« (ebd.) an der Produktion von innerer Sicherheit beteiligt sind. Somit betrifft innere Sicherheit nicht nur den Staat, sondern wird durch eine Vielzahl an gesellschaftlichen Akteuren mitbestimmt und auch produziert. LANGE weist an anderer Stelle (vgl. LANGE 2006: 132)außerdem zu Recht darauf hin, dass innere Sicherheit nicht statisch zu verstehen ist, sondern dass sich das gesamte System wandelt und benennt 
dabei den Prozess der Europäisierung und den Verlust des polizeilichen Monopols der Sicherheitsproduktion (ebd.: 132f.) als wichtigste Tendenzen.

Im Gegensatz zu vielen anderen Bereichen von Sicherheit ist innere Sicherheit sehr stark politisiert, was nicht nur an der Vielzahl der beteiligten Akteuere liegt, sondern auch an den verschiedenen, teilweise kontroversen politischen Positionen, die innere Sicherheit im Prozess der politischen Willensbildung beeinflussen. Da sich gerade in diesem sensiblen Bereich sich vielfältige Berührungspunkte zwischen Sicherheit und Freiheit ergeben vor allem, wenn versucht wird, innere Sicherheit durch Zwangsmaßnahmen zu garantieren oder wiederherzustellen, wird die innere Sicherheit zu einem wichtigen Aspekt im weiteren Verlauf dieser Untersuchung werden.

Unter äußerer Sicherheit im klassischen Sinn versteht man im Gegensatz dazu die Abwehr von Gefahren von außerhalb des Staatsgebietes, beispielsweise durch kriegerisches Verhalten anderer Staaten. Eine Vielzahl an staatlichen Aktivitäten kann zu äußerer Sicherheit beitragen. Dazu zählen beispielsweise militärische Potenziale oder auch die Integration in kollektive Bündnissysteme wie die NATO oder die EU. Durch "globale Vernetzung" (LEIDHOLD 1987:78) bekommen diese europäischen Kooperationen auch Relevanz für den Rest der Welt, vor allem wenn die Länder des Bündnissystems koordiniert handeln. Ergänzt werden die kollektiven Bündnissysteme durch bilaterale Abkommen zwischen Staaten. Die sicherheitspolitischen Bemühungen der Staaten verändern sich im Laufe der Zeit (für Deutschland u. a. bei RISSEKAPPEN 1988: 41-179), abhängig von internationalen Vorgaben und auch von innerstaatlichen Machtkonstellationen. In zunehmendem Maße spielen bei der äußeren Sicherheit nicht-militärische Themen wie etwa der Terrorismus oder die grenzübergreifende organisierte Kriminalität eine Rolle. Innere und äuße- 
re Sicherheit sind im Gegensatz zur öffentlichen Sicherheit (vgl. u.a. Art.13 und $35 \mathrm{GG}$ ) nicht juristische sondern politische Begriffe, die für eine organisatorische Aufteilung im Bereich Sicherheit stehen. Als Grundlage für diese Aufteilung kann man Art. 35 GG heranziehen, der die behördliche Amtshilfe regelt und den Ländern erlaubt, Einheiten der Bundespolizei und der Streitkräfte in bestimmten Fällen anzufordern. Artikel 87 a GG bildet in diesem Kontext das Gegenstück zu Artikel 35 und regelt die Einsatzbefugnisse der Bundeswehr, die im Verteidigungs- oder Spannungsfall und zur Abwehr einer drohenden Gefahr im Inneren eingesetzt werden kann:

»Zur Abwehr einer drohenden Gefahr für den Bestand oder die freiheitliche demokratische Grundordnung des Bundes oder eines Landes kann die Bundesregierung, wenn die Voraussetzungen des Artikels 91 Abs. 2 vorliegen und die Polizeikräfte sowie der Bundesgrenzschutz nicht ausreichen, Streitkräfte zur Unterstützung der Polizei und des Bundesgrenzschutzes beim Schutze von zivilen Objekten und bei der Bekämpfung organisierter und militärisch bewaffneter Aufständischer einsetzen. Der Einsatz von Streitkräften ist einzustellen, wenn der Bundestag oder der Bundesrat es verlangen.«

Das Grundgesetz sieht demnach vor, die Bereiche der inneren und äußeren Sicherheit grundsätzlich voneinander getrennt zu halten und definiert klar mögliche Szenarien, in denen eine Aufweichung dieses Trennungsprinzips ermöglicht wird.

Unter technischer Sicherheit versteht man nach MURCK (1970:78) auf der einen Seite die »Harmlosigkeit der Verwendung an sich gefährlicher Mittel«. Zuverlässigkeit und Kontrolle und damit verbundene Gefahrlosigkeit kennzeichnen diesen Bereich der technischen Sicherheit. Der Betrieb eines Atomkraftwerkes mit seinen Notfallkühlsystemen und Abschaltautomatismen ist ein typisches Beispiel für diesen Aspekt der technischen Sicherheit, die allerdings, wie man aus der Erfahrung des GAUs von Tschernobyl weiß, sehr schnell in 
Unsicherheit und Kontrollverlust mit gravierenden Folgen umschlagen kann. Auf der anderen Seite bezeichnet technische Sicherheit auch die "Zuverlässigkeit des technischen Mittels« und somit auch die »Voraussehbarkeit» seiner Leistung (MURCK 1970: 78). Durch die immer komplexer werdende Technik wird es immer wichtiger, dass die Überwachung der Sicherheit in den einzelnen technischen Abläufen automatisiert abläuft, was aber wiederum neue Probleme bei der letztendlichen Verantwortung und Supervision durch den Menschen aufwerfen kann. Technische Sicherheit wird unterstützt durch diverse Formen der Sicherheitstechnik, die bei der Gefahrenentstehung (z.B. Ersatzkühlsysteme in Atomkraftwerken), als unmittelbarer Schutz vor möglichen Gefährdungen (z. B. Airbags) oder bei der Gefahrenprävention (Hinweisschilder, Schutzkleidungspflicht) wirksam sind.

Wirtschaftliche Sicherheit kann man in enger Verbindung mit sozialer Sicherheit näher betrachten. Erstere bezeichnet einen Zustand, in dem finanzielle und materielle Ressourcen zeit- und anlassabhängig in adäquater Menge zur Verfügung stehen. Dies gilt sowohl für Individuen ${ }^{1}$ als auch für Organisationen wie zum Beispiel Unternehmen.

Soziale Sicherheit funktioniert auf der individuellen Ebene auf ähnliche Art und Weise, da auch hier finanzielle Faktoren bestimmend sind. Bestimmten Unsicherheiten wie beispielsweise der Verlust des Einkommens durch Krankheit, Elternschaft oder Unfall wird durch Versicherungen begegnet. Dabei

1 Die individuelle Lebenssituation erfordert für den Einzelnen Vorausschau, um das tägliche Leben zu bewältigen. Die Planung der verfügbaren Ressourcen und das Berücksichtigen von staatlicher und familiärer Unterstützung kann dazu beitragen, die unmittelbare Zukunft finanziell zu sichern (vgl. HUTHMANN/SCHWEITZER 2001). 
wird das finanzielle Risiko auf die beteiligten Versicherungsnehmer verteilt, die gemeinsam in einen Topf einzahlen. Bei Eintritt des Versicherungsfalles erhält der einzelne Betroffene aus diesem eine zuvor vertraglich geregelte finanzielle Kompensation, wobei wichtig ist, dass der Versicherungsfall nur bei einem geringen Teil der Versicherungsnehmer zeitgleich eintritt, da nur so das Versicherungssystem stabil und die individuellen Beiträge bezahlbar bleiben. Der Zustand der Sicherheit durch die Versicherung wirkt somit auf zwei Arten. Einerseits wird der von einem Versicherungsfall Betroffene unmittelbar finanziell entschädigt und andererseits gibt die Versicherung allen Versicherungsnehmern auf der Basis der Versicherungspolice ein Gefühl der Sicherheit, was zukünftige Ereignisse angeht. Die Intensität dieses subjektiven Sicherheitsgefühls durch eine Versicherung hängt auch davon ab, ob die Versicherungsnehmer sich freiwillig daran beteiligen.

Wirtschaftliche und soziale Sicherheit sind auch im Kontext von "Statussicherheit« (GLAESSNER 2002: 4) - bezogen auf Dimensionen sozialer Ungleichheit wie materieller Wohlstand, Macht, Prestige, Bildung, Arbeits-, Wohn-, Umwelt- und Freizeitbedingungen (vgl. HRADIL 1999: 27) - zu sehen. Wenn nämlich von wirtschaftlicher und sozialer Sicherheit die Rede ist, dann ist für die Menschen in der Regel auch der Erhalt, die Stabilisierung oder gar die Erhöhung des Status in Bezug auf die genannten Dimensionen ein wichtiger Faktor.

Rechtssicherheit ${ }^{1}$ beruht in der Bundesrepublik Deutschland auf Artikel 20 des Grundgesetzes, der in Zusammenspiel mit anderen Artikeln die verfas-

1 Eine ausführliche Zusammenstellung der einzelnen für den Rechtsstaat relevanten Grundgesetzartikel findet sich bei HESSELBERGER (1999: 180 f.) 
sungsrechtliche Grundlage für den Rechtsstaat darstellt. Drei zentrale Merkmale von Rechtssicherheit lassen sich (vgl. SACHS 2007: 806 ff.) festhalten: Der Grundsatz der Rechtsklarheit ist dabei ein wesentliches Merkmal. Jedes Gesetz muss sprachlich so eindeutig formuliert sein, dass kein Raum für unterschiedliche Interpretationen verbleibt. Diese Forderung nach Eindeutigkeit und die Komplexität der Gegenstände führt dazu, dass ein Großteil der Gesetze aus umfangreichen und ausführlichen Formulierungen besteht. In Folge dessen geht die erreichte sprachliche Exaktheit zu Lasten der allgemeinen Verständlichkeit und führt dazu, dass die Juristen gewissermaßen als Übersetzer der komplexen Fachsprache für den juristischen Laien fungieren müssen. Unter Bestimmtheit, dem zweiten wichtigen Merkmal, versteht man die graduelle Abstufung der Konkretisierung von Rechtsnormen von abstraktgenerellen Gesetzen über konkreter werdende Verordnungen bis zu eindeutigen einzelnen Rechtsakten. Die Beständigkeit der Gesetze ist das letzte wichtige Merkmal; sie soll das Vertrauen der Bürger in bestehende Regelungen stärken. Problematisch sind jede Form von Gesetzesänderungen, wenn sie für einzelne Betroffene eine Verschlechterung der Umstände bedeuten. Der Grundsatz nulla poena sine lege ist dabei bedeutsam, da er verhindert, dass rückwirkend bestimmte Verhaltensweisen unter Strafe gestellt werden können. Der Artikel 103 (2) des Grundgesetzes - „Eine Tat kann nur bestraft werden, wenn die Strafbarkeit gesetzlich bestimmt war, bevor die Tat begangen wurde.« - wirkt in diesem Sinne und stärkt neben den unmittelbar Betroffenen auch die Legislative demokratischer politischer Systeme, da dadurch sowohl der Exekutive als auch der Legislative die Möglichkeit genommen wird, willkürlich zu entscheiden. Von den aufgeführten Merkmalen von Sicherheit werden im weiteren Verlauf dieser Arbeit besonders die politische Sicherheit 
und die innere Sicherheit wichtig sein, obwohl auch die anderen behandelt werden. Das besondere Verhältnis von Freiheit und Sicherheit zeigt sich nämlich vor allem im Bereich dieser Dimensionen von Sicherheit, wo sich vor allem durch die Aktivitäten der beteiligten Behörden - unter besonderer Berücksichtigung der jeweiligen gesetzlichen Grundlagen - Berührungspunkte zeigen.

\subsubsection{Die Produzenten von Sicherheit}

Versteht man Sicherheit als die Abwesenheit von Risiken, Ungewissheit oder Unsicherheit, muss wohl konstatiert werden, dass Sicherheit in einer Gesellschaft nicht einfach als gegeben angenommen werden kann. Vielmehr erscheint es als gewiss, dass »Bedrohung und Unsicherheit (.) schon immer zu den Bedingungen menschlicher Existenz«(BECK 2007: 19) gehört haben, da eine Vielzahl an Faktoren unterschiedliche Formen von Unsicherheit produziert. Zum einen sind es die einzelnen Gesellschaftsmitglieder selbst, die sich riskant verhalten und andere gefährden. Dazu zählt sowohl der Raser auf der Autobahn, der sich und andere bedroht, als auch der Mitarbeiter im Atomkraftwerk, der zu Wartungszwecken Kühlsysteme umgeht und so einen GAU verursacht. Zum anderen lauern Risiken auch in Form von Ereignissen, die nicht am Verhalten einzelner festgemacht werden können, sondern gewissermaßen höhere Gewalt sind. Dazu zählen beispielsweise Hungersnöte, Naturkatastrophen oder Krankheitsepidemien, die Unsicherheiten in großem Rahmen erzeugen können. Nicht immer sind diese Risiken klar zu erkennen, oftmals vollziehen sich die Abläufe über längere Zeiträume hinweg und nicht immer kann man die Verantwortung eindeutig zuordnen. Der Versuch, Sicher- 
heit als Antwort auf bestimmte Risiken zu schaffen, ist in der Regel mit einigem Aufwand verbunden. In diesem Sinne kann man sie als ein Gut verstehen, das knapp und nicht frei verfügbar ist und das für die Teile einer Gesellschaft als potenziellem Abnehmer erst produziert werden muss. Man kann die verschiedenen Produzenten vom Individuum ausgehend ordnen.

Schon der Einzelne ist in der Lage, durch verschiedene Verhaltensweisen Sicherheit zu kreieren, indem er versucht, durch sein Verhalten Risiken zu vermeiden. Das Risiko, an einer bestimmten ansteckenden Krankheit zu erkranken, kann ganz einfach dadurch verringert werden, dass entweder bestimmte Gegenden gemieden werden oder dass durch Impfungen vorgesorgt wird. Zur Produktion von Sicherheit auf der individuellen Ebene zählen auch so simple Dinge wie das Anbringen eines Vorhängeschlosses an seinem Eigentum, um dieses vor unbefugtem Zugriff zu schützen. In erweiterter Form können auch andere Eigentumsformen mit vergleichsweise einfachen Maßnahmen sicherer gemacht werden. Die Fähigkeit, auf diese Art Sicherheit zu produzieren, hängt von den Ressourcen ab, auf die der Einzelne zugreifen kann. So hängt beispielsweise die Sicherung des eigenen Besitzes durch Überwachungsanlagen, Mauern, Schlösser und Türen in hohem Maße von den zur Verfügung stehenden finanziellen Mitteln ab. Auch in anderen Lebensbereichen kann Sicherheit vom Einzelnen produziert werden. Dazu zählt beispielsweise das Einzahlen in eine private Rentenversicherung oder das Schaffen von finanziellen Rücklagen als Formen der wirtschaftlichen Sicherheit. Im Bereich der technischen Sicherheit kann man sich neben einer Vielzahl von Maßnahmen beispielsweise durch den Erwerb von Autos mit moderner Sicherheitstechnik oder dem Verwenden von Rauchmeldern in Wohnhäusern absichern. 
Die Risikowahrscheinlichkeit, die den Grad an möglicher Sicherheit definiert, ist allerdings in den genannten Bereichen nicht immer evident und auch zuverlässig abzuschätzen. Im Bereich der politischen und der Rechtssicherheit kann der Einzelne nur in äußerst begrenzten Umfang - in dem er zum Beispiel bei der Polizei arbeitet und somit als Teil einer Behörde fungiert - Sicherheit für sich und Andere produzieren. An dieser Stelle wirkt in der Regel der Staat mit seinen verschiedenen Behörden und Einrichtungen als primärer Produzent der Sicherheit für die gesamte Gesellschaft (siehe Abbildung 3). Dies gilt gleichermaßen für die äußere Sicherheit und die innere Sicherheit. Gerade bei letzterer sind die verschiedenen Exekutiv-Behörden des Staates, die im demokratischen Staat auf der normativen Grundlage von Gesetzen operieren, entscheidende Ausführungsorgane. Nach ACHSNICH (U.a. 1997: 12) ist es das Ziel der aufgeführten Sicherheitsbehörden durch Maßnahmen wie dem unmit-
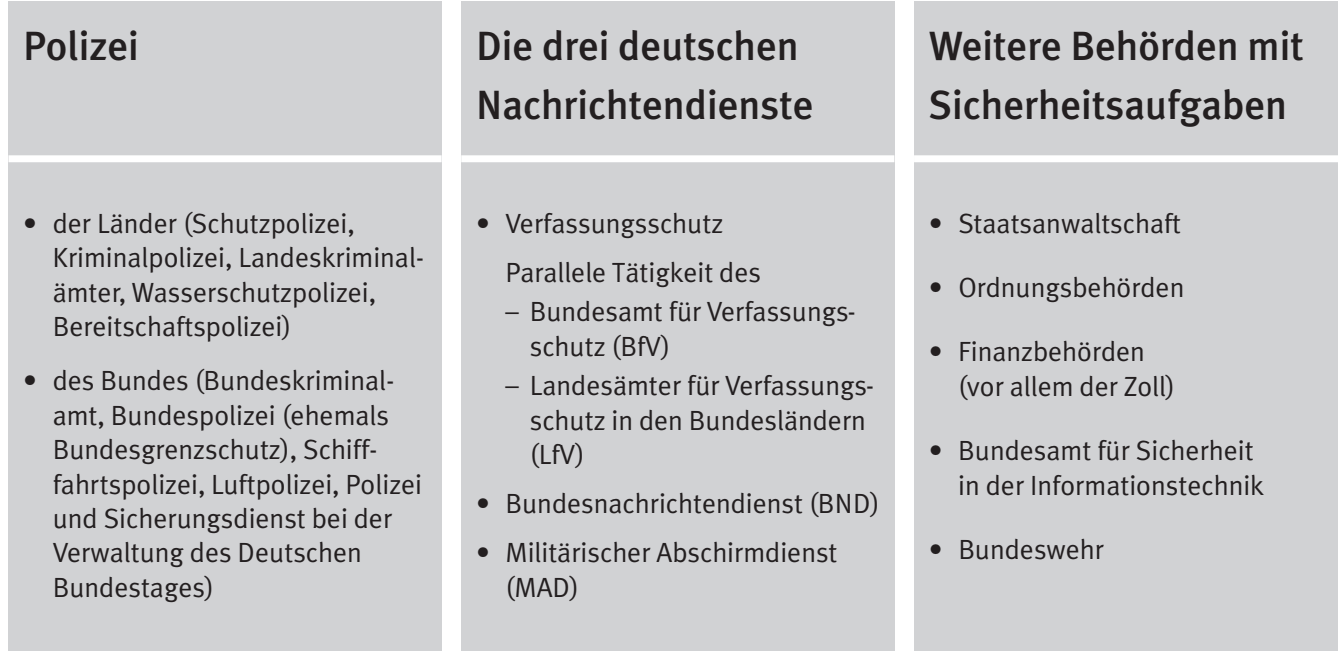

Abbildung 3 Überblick über deutsche Sicherheitsbehörden, ergänzt und abgeändert (vgl. ACHSNICH U.a. 1997: 6-8) 
telbaren Zwang (körperliche Gewalt) beziehungsweise der Strafverfolgung (auf der Basis des Strafrechts) der inneren Ordnung des Staates und dem gewaltlosen Umgang der Bürger (vgl.ebd.) zu dienen. Die alleinige Sanktionsmacht des Staates, der als übergeordnete »Berufungsinstanz« (MURCK 1989:6) bei Konflikten zwischen einzelnen Bürgern einer Gesellschaft eingreift und die Rechtsgüter schützt, ist ein entscheidendes Merkmal dafür, was einen Staat überhaupt ausmacht: „Staat soll politischer Anstaltsbetrieb heißen, wenn und insoweit sein Verwaltungsstab erfolgreich das Monopol legitimen physischen Zwangs für die Durchführung der Ordnungen in Anspruch nimmt« (WEBER 1976: 29). Dieses charakteristische Gewaltmonopol des Staates wird in der alltäglichen Praxis auf vielfache Weise aufgeweicht. Neben einzelnen Bürgern, die sich ebenfalls gewaltförmig verhalten, wird zudem in steigendem Maße die Produktion von Sicherheit delegiert.

Somit treten neben die verschiedenen staatlichen Behörden kleinere Organisationen, die ebenfalls Sicherheit produzieren sollen. Eine der gesetzlichen Grundlagen für die Tätigkeit von privaten Sicherheitsproduzenten sind die Ausnahmesituationen, in denen der Einzelne berechtigt ist, selbst Gewalt anzuwenden:

»bei Notwehr und Nothilfe ( $\S \S 227$ BGB, $32 \mathrm{StGB}, 15$ OwiG), in den gesetzlich geregelten Fällen des Notstandes ( $\S \S 228,904$ BGB, 34, 35 StGB), zum Schutz des Besitzers einer Sache ( $\S \S 858 \mathrm{ff} . \mathrm{BGB}$ ), zur Sicherung privatrechtlicher Ansprüche in Form der allgemeinen Selbsthilfe (§§ 229f. BGB), zur vorläufigen Festnahme eines Straftatverdächtigen (§ 127 Abs.1 StPO)« (ACHSNICH 1997: 101).

Indem diese eigentlich als Ausnahmen konzipierten Bestimmungen gleichsam organisiert werden, entsteht so, gewissermaßen von unten nach oben, privat produzierte Sicherheit. Gleichzeitig wird von oben nach unten ebenfalls von staatlicher Seite aus Sicherheit privatisiert. Dazu zählen alltägliche Dinge 
wie die Prüfung der Betriebssicherheit bei PKWs durch den TÜV oder die DEKRA, aber auch die Wahrnehmung von Aufgaben der Luftsicherheit durch die Deutsche Flugsicherung GmbH (vgl. ebd.: 100). Die so entstehende Produktion von Sicherheit durch private Träger mutet teilweise geradezu grotesk an, wenn die Bundeswehr als stark bewaffnete Organisation Unterstützung bei der Bewachung ihres Geländes durch rund 8000 Sicherheitsunternehmen erfährt (ebd.). Am Beispiel Bundeswehr wird deutlich, dass es die Ressourcen sind, die das Auslagern von Sicherheitsaufgaben an private Produzenten so interessant für die Beteiligten macht. Private Anbieter können bestimmte Aufgaben oftmals günstiger anbieten, was beim öffentlichen Anbieter von Sicherheit die Betriebskosten senkt. Wichtig dabei ist, dass beim Zusammenspiel von privaten und staatlichen Sicherheitsorganisationen wirtschaftlich defizitäre Sicherheitsaufgaben letztendlich nicht vernachlässigt werden.

Neben diese Problematik tritt eine weitere: Die privaten Anbieter von Sicherheit sorgen dafür, dass die individuelle Sicherheit davon abhängt, dass man sie sich auch leisten kann (vgl. ACHSNICH 1997: 101). MURCK präzisiert diesen Umstand, spricht aber sowohl von negativen als auch positiven Effekten (vgl. MuRCK 1980: 9). Als Beispiel für einen negativen Effekt kann ein Einkaufszentrum gelten, das mit Hilfe eines privaten Sicherheitsdienstes sicherer ist als ein anderes Geschäft, das sich keine solche Unterstützung leisten kann. Aus der Perspektive der beiden Unternehmen ist in diesem Sinne Sicherheit ungleich verteilt. Im gleichen Zug nützt das Sicherheitsstreben des Einkaufszentrums aber nicht nur der Firma, die es betreibt. Im Sinne eines positiven Effekts profitieren davon auch die Kunden und Besucher, die sich in einer Zone mit erhöhter Überwachung aufhalten können und damit sicherer 
sind. Die Privatisierung von Sicherheit, die von staatlicher Seite aus erfolgte, erschwert letztlich die Kontrolle durch den Staat. Besonders betroffen sind von diesem Kontrollverlust parlamentarische Gremien (vgl. WOHLFAHRT 2005: 56), denen durch das Outsourcen die Möglichkeit genommen wird, mit Hilfe von parlamentarischen Kontrollrechten Einfluss zu nehmen, da die entsprechende Sicherheitsaufgaben nicht mehr unmittelbar Teil der Exekutive sind. Eine Kontrolle der Ausgaben für Sicherheit ist damit en detail für die Kontrollgremien nicht mehr möglich, für die ausgelagerten Aufgaben verbleibt nur noch ein pauschaler Posten im Etat, womit es nicht mehr für die Legislative möglich ist, einzelne Kostenstellen zu hinterfragen.

\subsubsection{Sicherheit und Kontrolle}

Das Verständnis von Sicherheit als einem knappen Gut, das auf den Ebenen Staat, private Anbieter und den Individuen produziert wird, muss durch weitere Inhalte ergänzt werden, um ein angemessenes Verständnis zu ermöglichen. In enger Verbindung mit Sicherheit steht dabei der Begriff der sozialen Kontrolle, den man nach LAMNEK wie folgt definieren kann: „Der sozial relevante Mechanismus, der der Durchsetzung der Normen dienen soll, wird in der Soziologie als soziale Kontrolle bezeichnet« (LAMNEK 1979: 20). Der Grad an Sicherheit in einer Gesellschaft hängt ganz entscheidend davon ab, ob ihre Mitglieder entsprechend der Verhaltenserwartungen sozial handeln. Viele von der Norm abweichende Verhaltensweisen produzieren Unsicherheiten und Risiken. Ein einfaches Beispiel dafür ist ein Geisterfahrer, der gegen die Straßenverkehrsordnung verstößt, indem er die falsche Seite einer Straße benützt und somit die anderen Verkehrsteilnehmer gefährdet. Doch nicht alle von der 
Norm abweichenden Verhaltensweisen kreieren ein Risiko für Leib und Leben. Oftmals sorgt das abweichende Verhalten lediglich dafür, dass wir uns unsicher fühlen. Der Grund dafür ist, dass Situationen plötzlich an Komplexität gewinnen und nicht mehr nach bestimmten Automatismen ablaufen können. Treffen wir beispielsweise in einer belebten Fußgängerzone auf nackte Passanten, so reagieren wir in der Regel erstaunt oder fühlen uns belästigt. Die gesamte Situation ist für die Beobachter von Unsicherheit geprägt und Verhaltensweisen wie Blickkontakt, das Wahren gewisser Distanzen und alle Reaktionen bei einem direkten Kontakt stehen zur Disposition. Soziale Kontrolle wirkt dieser Situation entgegen und versucht mit Hilfe von positiven und negativen Sanktionen konformes Verhalten herbei zu führen. Sanktionen wirken dabei zukunftsorientiert, da bereits geschehene Brüche von Normen, die Bestrafung nach sich zogen, durch die Sanktion nicht rückwirkend beeinflusst werden können. Stattdessen können nach LAMNEK (1979: 20) Sanktionen soziologisch nur sinnvoll sein, wenn sie bewirken, dass sowohl der individuell Betroffene als auch alle potenziellen Abweichler von der Norm sich stärker an dieser orientieren. Erfolg ist ein wichtiges Kriterium für die Bewertung der Effektivität einer Sanktion, wobei nicht nur die Art und Weise der Sanktion, sondern auch die Wahrscheinlichkeit, dass überhaupt sanktioniert wird, eine wichtige Rolle spielt (vgl. ebd.).

Nach COHEN kann man soziale Kontrolle nach verschiedenen Kontrollstilen und Organisationsformen klassifizieren (vgl. COHEN 1993: $214 \mathrm{ff}$., siehe Abbildung 4). COHEN greift bei seiner Darstellung der Organisationsformen sozialer Kontrolle auf eine Typologie von HorowITzS »Logic of Social Control« (vgl. Horowitz 1990) zurück und unterscheidet sie nach der Anzahl der Beteiligten. Unilaterale Kontrolle umfasst Aktivitäten, die lediglich einen Betei- 

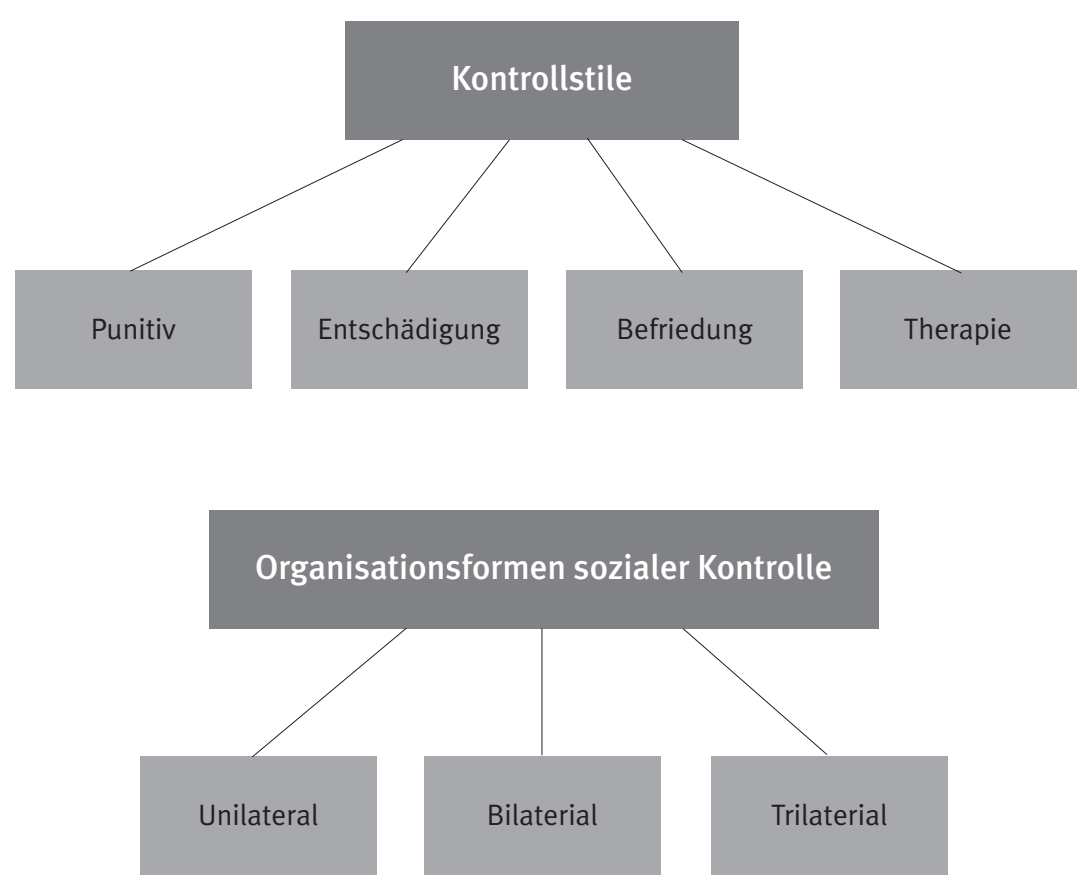

Abbildung 4 Stile und Organisationsformen sozialer Kontrolle nach CoHEN (1993: 214-217)

ligten kennen. Mögliche Verhaltensweisen sind dabei das Vermeiden oder das Verlassen bestimmter Situationen, sowie bestimmte Formen der Selbsthilfe (vgl. COHEN 1993: 217). Wird soziale Kontrolle bi- und trilateral organisiert, dann treten weitere Beteiligte als Betroffene und im letzteren Fall als dritte, übergeordnete Kontroll- und Schlichtungsinstanzen hinzu.

Für die verschiedenen Organisationsformen der sozialen Kontrolle unterscheidet CoHEN (ebd.) vier unterschiedliche Kontrollstile. Unter dem punitiven Kontrollstil versteht er das Ausüben von Zwang zur Durchsetzung sozialer Kontrolle. Neben Opfer und Täter tritt eine zusätzliche dritte Instanz, die die verantwortliche Person bestraft. Nach CoHEN (1993: 214) dominiert dieser Stil 
die Wahrnehmung von sozialer Kontrolle. Besonders bei schweren Verbrechen findet sich häufig der Ruf nach härteren Strafen, die abschrecken sollen. Dem entgegengesetzt wirken Entschädigung und Befriedung abseits von Strafe und Bestrafung. Im Mittelpunkt stehen bei ersterem die Kompensation des Schadens, welchen das Opfer erlitten hat, durch den Täter. Im Fall von Befriedung steht die Unterscheidung zwischen Opfer und Täter nicht mehr im Mittelpunkt, sondern im Stile einer Schlichtung wird versucht, durch Verhandlung eine Wiedergutmachung zu erreichen. Der therapeutische Kontrollstil hat das erklärte Ziel, zu helfen (vgl. ebd.: 216) und seine Anwender verwahren sich oftmals gegen den Vorwurf, sie würden soziale Kontrolle ausüben (vgl. ebd.). Trotzdem, so CoHEN weiter, sei auch der therapeutische Stil als eine Art der sozialen Kontrolle anzusehen, da durch die »Konstruktion neuer therapeutischer Kategorien« (ebd.) neue Formen von Abweichung und Normalisierung gebildet und festgelegt werden. Es lohnt sich, den Begriff der sozialen Kontrolle daher nochmals definitorisch aufzugreifen und zu erweitern. Singelnstein und Stolle (2006) definieren soziale Kontrolle etwas anders und stellen alternative Klassifikationen vor:

»Sozialkontrolle als Begriff umfasst unabhängig von der jeweiligen Gesellschaftsform sowohl staatliche als auch private Mechanismen und Prozesse, mit denen eine Gesellschaft oder eine sonstige soziale Gruppe versucht, ihre Mitglieder dazu anzuhalten, den von ihr aufgestellten Normen als Verhaltensanforderungen Folge zu leisten und soziale Integration herzustellen« (SINGELNSTEIN/STOLLE 2006: 11).

Die Autoren unterscheiden zwei Ebenen der sozialen Kontrolle, nämlich die »Ebene der Normgenese« (ebd.) von der »Durchsetzungsebene« (ebd.). Ebenfalls relevant ist die Differenzierung zwischen formellen sozialen Normen, die schriftlich kodifiziert beispielsweise in der Form von Gesetzen werden und 
informellen Normen, die »auf Brauch, Sitte, Tradition und Moral« (ebd.: 12) beruhen. Für das Verständnis wichtig ist auch die Unterscheidung in professionelle und private soziale Kontrolle. Unter ersterem fassen SINGELNSTEIN und Stolte die staatlichen und kommerziellen Agenten sozialer Kontrolle zusammen und stellen ihnen die private gegenüber, die »im sozialen Nahraum« (ebd.) ausgeübt wird. Die Mittel »wie Kritik, Spott, Missbilligung, Abbruch der Sozialbeziehungen oder auch physischer Gewalt« (ebd.), die von den Autoren angegeben werden, kann man noch um verbale Sanktionen allgemein, Augenkontakt oder Berührungen erweitern. Die Ausübung der sozialen Kontrolle im Nahraum wird so zum Teil der Sozialisation.

Peters stellt im Zusammenhang mit sozialer Kontrolle fest, dass, misst man ihren Erfolg am Potenzial zur Verminderung abweichenden Verhaltens, es wenig Anhaltspunkte dafür gibt, dass sie überhaupt Erfolg hat (PETERS 1989: 175). Eine empirische Prüfung fällt in diesem Kontext schwer, da abweichendes Verhalten und noch weniger konformes Verhalten präzise fassbar sind. Interessant in diesem Zusammenhang ist die Definition sozialer Kontrolle, wie sie SCHEERER (2000: 167) vornimmt:

\footnotetext{
»Warum sollten wir soziale Kontrolle eigentlich nicht als Ensemble all dessen definieren, was unerwünschtes Verhalten verhindern soll und/oder faktisch verhindert (auch der Versuch der Verhinderung kann ein Kontrollverhalten sein) - sowie all dessen, was auf unerwünschtes Verhalten reagiert (ob das nun funktioniert oder nicht)?«
}

Im Mittelpunkt steht dabei die Art und Weise wie und ob abweichendes Verhalten wahrgenommen wird und wie darauf reagiert wird. Der Wirkungsgrad, den soziale Kontrolle erreicht, gibt Aufschluss über den Grad an Sicherheit in einer Gesellschaft, zumindest was die Sicherheit angeht, die sich aus dem sozialen Handeln ihrer Mitglieder ergibt. 
Drei wichtige Gesellschaftsmodelle lassen sich heranziehen, um die besonderen Umstände der sozialen Kontrolle zu präzisieren und exakt zu erfassen. Das erste ist das Modell der Disziplinargesellschaft von Foucault, das von einer Vielzahl von neueren Modellen aufgegriffen wird und ihnen als Grundlage dient. Es soll hier darauf verzichtet werden, alle gesamten Gedankengänge Foucaults ausführlich zu erörtern, stattdessen soll der Schwerpunkt auf den besonderen Aufbau der Disziplinargesellschaft gerichtet werden, den Foucault in seinem Werk »Überwachen und Strafen - Die Geburt des Gefängnisses« plastisch beschreibt. Er greift dabei auf das so genannte Panopticon von Bentham zurück (vgl. FouCAuLt 1976: 256 ff.), einer räumlichen Struktur, die ein »ideales« Gefängnis beschreibt: »An der Peripherie ein ringförmiges Gebäude; in der Mitte ein Turm, der von breiten Fenstern durchbrochen ist, welche sich nach der Innenseite des Ringes öffnen, das Ringgebäude ist in Zellen unterteilt, von denen jede durch die gesamte Tiefe des Gebäudes reicht; sie haben jeweils zwei Fenster, eines nach innen, das auf die Fenster des Turmes gerichtet ist und eines nach außen, so daß die Zelle auf beiden Seiten von Licht durchdringen wird« (ebd.: 256f.). Aus der Mitte des Überwachungsturmes ist jede Zelle einsehbar und überwachbar und das mit einem relativ geringen personalen Aufwand. Aus dieser räumlichen Anordnung ergibt sich ein »permanenter Sichtbarkeitszustand» (ebd.: 258), alle Gefangenen müssen kontinuierlich damit rechnen beobachtet und kontrolliert zu werden. Dieser Mechanismus garantiert bei Foucault die Disziplin der Beteiligten, die durch eine »automatisches Funktionieren der Macht« (ebd.) ermöglicht wird. FouCAULt erweitert diese architektonische Gestaltung eines Gefängnisses auch auf andere totale Institutionen wie Kasernen, Schulen, Kirche, Familie oder Krankenhäuser (ebd.: 261) und darüber hinaus zu einem Modell der 
Gesellschaft. Die Segmentierung des Raumes in voneinander isolierte Bereiche, mit entsprechenden Macht- und Disziplinstrukturen sieht er als gesellschaftlich verbreitete Realität. Für inn repräsentiert sie »die Perfektionierung der Machtausübung« (ebd. 265), auch weil diese Machtausübung gewissermaßen entpersonalisiert ist; die Macht liegt in der Struktur des panoptischen Aufbaus und es ist nicht entscheidend, wer sie konkret ausübt. Das Panopticon wird letztlich bei Foucault zu einer Metapher, die den gesamten von Disziplinen durchsetzten (ebd.: 268) gesellschaftlichen Aufbau bis in feinste Verästelungen widerspiegelt. Für bestimmte Entwicklungen in unserer Gesellschaft, wie etwa die akustische Wohnraumüberwachung oder die Videoüberwachung an öffentlichen Plätzen erhalten seine Überlegungen einer geradezu beklemmende Aktualität. Als gesellschaftliches Modell wurde der Disziplinargesellschaft aber schon kurze Zeit später das Modell der Kontrollgesellschaft zur Seite gestellt - verfasst von Gilles Deleuze, der Foucault in Freundschaft verbunden war und dessen Kontrollgesellschaft mit den Gedankengängen Foucaults in enger Verbindung steht. Deleuze sieht die von Foucault angeführten totalen Institutionen und vor allem auch den Staat (vgl. DeLEUzE 1993: 254) als Gebilde, die nur durch andauernde Reformen künstlich am Leben erhalten werden: »Eine Reform nach der anderen wird von den zuständigen Ministern für notwendig erklärt: Schulreform, Industriereform, Krankenhausreform, Armeereform, Gefängnisreform. Aber jeder weiß, dass diese Institutionen über kurz oder lang am Ende sind. Es handelt sich nur noch darum, ihre Agonie zu verwalten und die Leute zu beschäftigen, bis die neuen Kräfte, die schon an die Türe klopfen, ihren Platz eingenommen haben.« (ebd.). Die neuen Kräfte sind nicht mehr nur staatliche Einrichtungen, sondern auch private Einrichtungen. Lindenberg und SCHMIDT-SEMISCH greifen dieses Phäno- 
men auf und stellen in diesem Zusammenhang einen »neuen Trend zur Segmentierung von Kontrolle« (Lindenberg/Schmidt-Semisch 1995: 9) im Sinne einer »Vermehrung privater und privat organiserter Kontrollsektoren« (ebd.: 10) fest, welche das System der disziplierenden Kontrolle ergänzen. Das Panopticum zerbricht und weicht einem System »sektoralisierter Kontrollräume« (ebd. 11), das aber für den Einzelnen durchlässig ist. Die Autoren gebrauchen in diesem Zusammenhang das Bild der "Waben in einem Bienenstock» (ebd. 10), die allerdings untereinander verbunden und nicht abgegrenzt sind. Die staatlichen Wächter in diesem Gebilde patroullieren nurmehr and den durchlässigen Grenzen (ebd.: 11) und greifen nur noch bei übergreifenden Bedrohungen ein. Ein klassisches Beispiel dafür ist die Tätigkeit von Sicherheitspersonal beispielsweise in Diskotheken, die auch relativ autonom arbeiten und erst in vordefinierten Situationen Polizeibehörden mit hinzuziehen. Ein wichtiges Motiv für die Kontrollgesellschaft ist das Streben nach Sicherheit, die über eine abgestufte Kontrolle devianten Verhaltens sichergestellt werden soll. Der angestrebte Grad an Sicherheit ist dabei in den unterschiedlichen Segementen unterschiedlich ausgeprägt.

Das Modell der Sicherheitsgesellschaft knüpft hier an, erweitert aber den Kontrollbegriff erheblich und gibt Sicherheit einen neuen Stellenwert. Nach Legnaro zeichnet sich - ähnlich wie bei der Kontrollgesellschaft - eine Sicherheitsgesellschaft dadurch aus, »daß nicht nur staatliche, sondern allmählich und in stetig steigendem Ausmaß auch private Akteure an der Produktion von Sicherheit teilnehmen, daß die Überwachung nicht nur dem Staatsschutz im engeren Sinne gilt, sondern Aktivitätskontrollen von allen Bürgern (...) mit dem Ziel der Risikominimierung nicht nur eine staatliche Aufgabe ist, sondern eine permanente gesellschaftliche Anstrengung, ein Régime des täglichen sozialen 
Lebens (LEGNARO 1997: 272). Im Mittelpunkt steht die Produktion von Sicherheit, nun aber nicht mehr nur als Schutz vor devianten Verhaltensweisen, sondern zur »Wiederherstellung von sozialer Ordnung als solcher« (ebd.). Um dies zu ereichen, sind "soziale und technische Kontrollmechanismen« (ebd.), wie Legnaro sie nennt, vielfältig in den Alltag integriert. Dahinter steht der Versuch, Sicherheit in großem Stile zu etablieren, was neben dem Wissen um bestimmte Risiken auch ein großes Gefährdungsbewusstein in der Bevölkerung als Voraussetzung benötigt (vgl. ebd.: 273). Dadurch wird Sicherheitsgesellschaft zu etwas Umfassenderen als die Kontrollgesellschaft. LEGNARO benennt drei Elemente der Sicherheitsgesellschaft in diesem Kontext (vgl. ebd.: 274 ff.). Zum einen verändert sich die Zielrichtung der Kontrollmechanismen. Nicht mehr einzelne Personen sind die Ziele, stattdessen gilt die Kontrolle bestimmten Räumen. Als Beipiel nennt er dafür unter anderem die Schleierfahndung in Bayern, wo nun in einem $30 \mathrm{~km}$ breiten Korridor kontrolliert wird (ebd.). Diese Entpersonalisierung der Kontrolle führt hin zu verdachtsunabhängigen Ermittlungen, welche gewissermaßen schon im Vorfeld der Tat ansetzen. Polizeiarbeit wird somit autonomer, geht weg vom Reaktiven und wird "proaktiv« (ebd.: 276) An dieser Stelle ist die Sicherheitsgesellschaft eng verbunden mit der Präventionsgesellschaft - von ihr wird an anderer Stelle in dieser Untersuchung noch berichtet werden. Auch die verschiedenen Räume werden kontrolliert und abgesichert. Der gängige Mechanimus ist dabei der der Reglementierung - Exklusion oder Inklusion des Zutritts zu bestimmten Räumen, der oftmals auch mit einer (digitalen) Zugangsberechtigung zu erreichen sind (etwa die Räumlichkeiten einer Bank, die sich nur durch eine entsprechende Bankkarte öffnen lassen oder auch Platzverweise gegen bestimmte Bevölkerungsgruppen wie Obdachlose). 
LEGNARO räumt abschließend ein, dass es noch verfrüht sei, von einer vollständig etablierten Sicherheitsgesellschaft zu sprechen (vgl. ebd. 281), da Sicherheit noch nicht der dominierende Auslöser für soziale Interaktionen geworden ist. Stattdessen sieht er Sicherheitsgesellschaft überall dort, wo mit Hilfe von Kontrolle versucht wird, subjektive und objektive Risiken und Gefährdungslagen vorher zu sehen und ihre »Eintrittswahrscheinlichkeiten« (ebd.: 281) zu minimieren.

Singelnstein und Stolle ordnen die eingesetzen Mechanismen und Techniken sozialer Kontrolle, die im Rahmen der Sicherheitsgesellschaft relevant sind, nach verschiedenen Aspekten. Dazu zählen sie unter anderem so genannte »Techniken und Prozesse der Selbstführung« (SINGELNSTEIN/ STOLLE 2006: 56), bei denen augenscheinlich kein äußerer Zwang zu erkennen ist. Die Konformität entsteht gewissermaßen aus dem Individuum selbst heraus. Selbstverantwortung und Vorsorge sollen es dem Einzelnen ermöglichen, selbst Risiken zu minimieren. Ergänzend dazu werden »Techniken instrumenteller Kontrolle« (ebd.: 58) eingesetzt, die selbst unmittelbar für die Einhaltung von Normen (vgl. ebd.) sorgen. Maßnahmen wie Videoüberwachung, Einzäunungen oder elektronische Fußfesseln werden dabei unter anderem von den Autoren benannt (ebd.: 59). Ihnen gemein ist, dass sie keine Reaktion auf ein abweichendes Verhalten sind, sondern die Umwelt so verändert wird, das Abweichung bereits im Vorfeld unterbunden wird (ebd.). Dieser Versuch, den öffentlichen Raum gewissermaßen im Vorfeld zu beherrschen, birgt in Kombination mit modernen Techniken die Gefahr einer andauernden Ausweitung, zumindest dann wenn der Bedarf an Risikominimierung nicht endet. Bestimmte Kontrollinstrumente wie zum Beispiel der genetischen Fingerabdruck oder das Erfassen biometrischer Daten sind Kandidaten für eine 
solche Entwicklung, vor allem wenn die Möglichkeit besteht sie mit anderen Instrumenten der Kontrolle zu koppeln: Im Bereich der Biometrie gilt dies für das Zusammenspiel mit optischen Erfassungssystemen, denen damit die technische Möglichkeit gegeben wird, Personen nicht nur optisch zu erfassen, sondern sie auch gleich anhand biometrischer Daten zu identifizieren.

Ergänzt werden die zwei oben erwähnten Aspekte um die klassische disziplinierende Intervention, die in Form von strafrechtlichen Sanktionen weiter fortbesteht und die »das noch bestehende Rumpfgerüst sozialer Normen im engeren Sinne« (ebd.: 63) stabilisieren sollen. SINGELNSTEIN und STOLLE weisen darauf hin, dass das dahinterstehende System aus Strafverfolgungsbehörden als Struktur sehr stabil seien und dass die angewandten Techniken für eine große Anzahl an Delikten auch in der Sicherheitsgesellschaft adäquate Strategien liefert. Ausschlussstrategien werden von den Autoren als letzte Technik der Sicherheitsgesellschaft genannt. Gemeint ist damit ganz einfach der Versuch, bestimmte Personen und Personengruppen aufgrund einer »Klassifizierung« (ebd.: 65) von bestimmten Räumen fernzuhalten. Dazu zählt der Platzverweis als leichteste Form ebenso wie ein Gefängnisaufenthalt oder auch die Sicherungsverwahrung. Gerade letztere ist ein Phänomen, das für die Sicherheitsgesellschaft typisch ist, steht es doch für den Freiheitsentzug ohne eine dazugehörigen Straftat, sondern einfach aus der Einschätzung einer angenommene Gefährdung heraus. Das unterstellte Sicherheitsrisiko führt dazu, dass der Gefängnisaufenthalt erheblich verlängert werden kann. Zusammengefasst kann man sagen, dass die angesprochenen Elemente der Sicherheitsgesellschaft parallel wirken und sich oftmals auch gegenseitig ergänzen, wobei die verschiedenen Maßnahmen mit der Exklusion als letztem Ausweg auch abgestuft hintereinander geschaltet denkbar sind. 


\subsubsection{Sicherheit und Risiko als menschliche Grundbedürfnisse?}

Auf den ersten Blick erscheint es, dass das Bedürfnis der Menschen nach Sicherheit ein umfassendes Phänomen ist. In der Tat benötigen Menschen bei der Bewältigung ihres Alltags Sicherheit. Politische, technische, soziale und wirtschaftliche Sicherheit wird begleitet von Sicherheit im Sinne von Gewissheit. Wissen, das zum Handeln ausreicht (vgl. KAUfMANN 1970: 93), ist für letztere eine wichtige Bedingung. Institutionen und feste Strukturen bilden das Gerüst, auf dem das tägliche soziale Handeln aufbaut. Viele tägliche Abläufe werden dadurch automatisiert und müssen nicht ständig neu ausgehandelt werden. Die meisten Menschen leben heute sicher, ohne andauernd Gefährdungen für Eigentum, körperliche Unversehrtheit und Leben befürchten zu müssen. Auf dieser Basis an Sicherheit kann der Einzelne sein Leben im Zusammenspiel mit anderen weiter entfalten. Das System der sozialen Kontrolle ist darauf ausgelegt, Konformität zu etablieren. Der Versuch, Sicherheit durch soziale Kontrolle herzustellen, bedeutet für eine Gesellschaft enorme Kosten - gerade für die sozialen Absicherungssysteme im Bereich der inneren Sicherheit. So wurden im Bundeshaushalt von 2006 alleine für die Bundespolizei rund 2,08 Mrd. Euro veranschlagt (BMF 2006: 338) und man kann des Weiteren davon ausgehen, dass die Kosten für die Bundesländer als wichtigste Träger der Polizei ein Vielfaches davon betrugen. Ähnliches gilt auch für den Justizbereich (Gerichte, Staatsanwaltschaften, Justizvollzugsanstalten) in den Ländern, der zum Beispiel den bayerischen Landeshaushalt 2006 mit 1,6 Mrd. Euro (vgl. StMF 2006: 50) belastete. Dennoch existiert auf der Ebene der Individuen aber durchaus die Motivation, gezielt unsicher und riskant zu handeln. Damit ist einerseits der große Bereich an Handlungen 
gemeint, in dem Menschen unabsichtlich fahrlässig handeln und sich so Risiken aussetzen ${ }^{1}$, ebenso wie kriminelle Handlungen. Vielmehr gehören dazu auch »Leistungs- und Mutproben« (SCHLESKE 1977: 107),

»für die bestimmte Handlungsmuster charakteristisch sind: die Überwindung (natürlicher) Hindernisse (...), die Bemeisterung exponierter Raumlagen (...), die Manipulation und Beherrschung von Geschwindigkeit und/oder ungewöhnliche Arten der Fortbewegung (oder) der Zweikampf mit menschlichen oder tierischen Gegnern (...) (ebd.)«.

BRETON stellt in diesem Zusammenhang im Jahre 1995 eine "grundsätzlich wachsene Risiko- und Abenteuerlust (.) auch beim Durchschnittsbürger« (BRETON 1995: 10) fest und benennt dabei unter anderem Orientierungsläufe, Raids, Tiefschneefahren und vieles mehr. Aus heutiger Sicht lassen sich die Extreme und Risikosportarten fast schon beliebig steigern: Statt Tiefschneefahren abseits markierter Pisten treibt es die Sportler zum Steilwandskifahren und Orientierungsläufe finden seit 1994 zuweilen in Form eines Marathons am Nordpol statt (vgl. LoHMANN 2002). Für den geneigten Extrem-Risikosportler findet sich heute noch das so genannte Freesoloklettern - damit ist das Klettern einer Route ohne technische Hilfsmittel und Sicherung gemeint, das als »deep water free solo« gerne auch über einer Wasseroberfläche ausgeführt werden darf. Ausdauersportler können sich zudem einen so genannten IronMan-Triathlon antun, der den Sportler mit 3,8 Kilometer Schwimmen, 180 Kilometer Radfahren und als Abschluss noch mit einem klassischen Marathon über 42,195 Kilometer Laufen konfrontiert und körperlich bis an die Grenzen

1 An dieser Stelle soll der Selbstmord als ein Sonderfall sozialen Handelns außen vor gelassen werden, da er sowohl Elemente des absoluten Sicherheitsverlustes mit Elementen völliger Kontrolle vereint. 
belastet. Wer damit noch nicht ausgelastet ist, kann sich auch dem Race Across America stellen, das für die radfahrenden Teilnehmer ein Zeitlimit von 12 Tagen und 5 Stunden für eine Durchquerung der Vereinigten Staaten von Amerika von Küste zu Küste setzt (vgl. raceacrossamerica.org). Für die weniger Trainierten, die ihr Risiko abseits körperlicher Ausdauerleistungen suchen, warten die Disziplinen Vulkanbesteigung, Höhlenerforschung oder Bungeejumping, das heute auch gerne wegen der größeren Höhe im Korb eines Fesselballons seinen Anfang nimmt. Die Lust am Risiko, wie BRETON sie nennt (vgl. BRETON 1995) und das Suchen nach körperlichen Extremerfahrungen scheinen das genaue Gegenteil von Sicherheit zu sein und die Extremsportarten geraten durch ihre Grenzerfahrungen in die Nähe zu Sterben und Tod (vgl. u.a. bei MüLLER 2007).

Die heutigen Extremsportler teilen mit früheren Abenteurern und Entdeckern wie Livingston (Afrika), Amundsen (Nordpol) oder auch ARMSTRONG (Mond) eine ganze Reihe von Dingen, die sie motivieren, aus der Sicherheit auszubrechen. Es winken Ersatzbelohnungen in Form von sozialer Anerkennung, Geld - beziehungsweise Werbeverträge in heutiger Zeit -, Ehre, Selbstüberwindung, Adrenalin und Nervenkitzel, Aufmerksamkeit und Ruhm, wenn sie ihre spektakulären Höchstleistungen erbringen. Der Verlust an individueller Sicherheit wird damit für die Extremsportler und Abenteurer anscheinend kompensiert. Für die jeweilige Gesellschaft wirkten vor allem die Abenteurer und Entdecker oftmals innovierend. Von den persönlichen Risiken der Entdecker (zum Beispiel in der See- und Raumfahrt) profitierten heute ebenfalls andere, indem beispielsweise neue Märkte erschlossen und neue Technologien entwickelt wurden. In abgewandelter Form trifft das auch auf die heutigen Extremsportler zu, die immer wieder aufzeigen, dass das Ausreizen 
individueller Grenzen möglich ist, wobei auch deutlich wird, dass dafür auch Preise in Form von enormer Anstrengung, Verletzung oder gar Tod zu bezahlen sind.

Das persönliche Verhältnis zur Sicherheit stellt sich aus der Perspektive der Extremsportler bisweilen anders dar als für Außenstehende, welche die erbrachten riskanten Leistungen kopfschüttelnd, anerkennend oder auch neidisch zur Kenntnis nehmen. Gerade in Extrembereichen wie dem Sportklettern, dem Motorsport oder dem Skydiving widmen sich viele Sportler ausführlich ihrer persönlichen Sicherheit, indem sie versuchen, ihren persönlichen Trainingsstand, ihre Technik und die Ausrüstung zu optimieren. Risiko soll sich auf diese Weise in dem begrenzten Rahmen, den die Unsicherheitsfaktoren lassen, in kalkulierbares Risiko verwandeln.

\subsubsection{Wissen - Objektive und subjektive Sicherheit}

Das kalkulierte Risiko des Extremsportlers, der versucht, mit Hilfe verschiedener Maßnahmen, Sicherheit zu produzieren, ist ab und an trügerisch, vor allem, wenn die Natur nicht mitspielt. Spektakuläre Unfälle im Hochgebirge, bei denen Bergsteiger unterschiedlicher Leistungsfähigkeit und alpiner Erfahrung sich selbst überschätzen und von Lawinen, Steinschlag oder Wetterumschwüngen überrascht werden, sind bekannte Beispiele dafür, wenn scheinbare Sicherheit in extremes Risiko umschlägt. Es gibt also erhebliche Unterschiede zwischen der tatsächlichen Sicherheitslage und der persönlichen Empfindung von Sicherheit und Risiko. Es erscheint deshalb sinnvoll, Sicherheit analytisch nach einer objektiven und einer subjektiven Ebene zu unterscheiden. Ähnlich wie bei der Ausübung der Eigenkontrolle zur Absicherung 
von Freiheit ist dabei Wissen ein entscheidender Faktor. Sicherheit im objektiven Sinn meint die Sicherheit, die vollkommen von objektiven Randbedingungen abhängt und die durch Wahrscheinlichkeiten bedingt wird. Betrachten wir zur Illustration ein einfaches Würfelereignis. Bei einem sechsseitigen LaplaceWürfel beträgt die Wahrscheinlichkeit, eine 6 zu würfeln, genau ein Sechstel. Sie ist auch bei jedem weiteren Wurf identisch. Insofern kann der Spieler sich bei jedem Wurf sicher sein, dass seine Chancen exakt gleich bleiben. Da die Komplexität von menschlichen Handlungen wesentlich höher ist als die eines Würfelereignis, können die objektiven Bedingungen in der Praxis verborgen sein und erst durch das individuelle Wissensniveau aufgedeckt werden: Erst mit einem bestimmten mathematisch-stochastischen Wissen können wir zum Beispiel einschätzen, mit welcher Wahrscheinlichkeit wir im Lotto (6 aus 49) gewinnen oder auch nicht.

Die reine quantitative Erhöhung des Wissens kann somit dabei helfen, die objektiven Randbedingungen eines bestimmten Ereignisses zu erfassen. Problematisch ist aber dabei, dass dies im Grunde nur in der Rückschau auf bestimmte Ereignisse funktionieren kann. Um beim Würfelereignis zu bleiben: Das Wissen um die Wahrscheinlichkeiten gibt uns noch keine Auskunft darüber, welche Zahl beim nächsten Wurf tatsächlich gewürfelt wird, wir wissen nur, dass bei einer relativ hohen Zahl von Würfelereignissen man sich langsam von den tatsächlichen Würfelergebnissen her dem theoretisch konstruierten Verhältnis von 1 zu 6 annähert. Zusammengefasst kann man sagen, dass die objektive Sicherheit eines Menschen von den Randbedingungen abhängt. Seine wirtschaftliche und soziale Sicherheit hängt ab von seinem Besitz, seinen Wohnverhältnissen, seiner Arbeitsstelle und von der Art, wie er für die Zukunft vorgesorgt hat. Seine physische Sicherheit hängt ab von seinen 
körperlichen Voraussetzungen, seinem Alter, seinem Gesundheitszustand und der Art und Weise, wie er Konflikte bewältigen kann. Sämtliche objektive Gegebenheiten sagen nichts darüber aus, wie sich die Sicherheitslage verändern wird: Objektive Sicherheit ist eine Momentaufnahme, die auf Daten der Vergangenheit beruht. Sie wird durch den Einzelnen um einen »psychologischemotionalen Zugang" (WIDMER 1995: 11) ergänzt. Neben die objektive Sicherheit tritt mithin das Sicherheitsgefühl, das festlegt, wie die objektive Sicherheitslage jeweils interpretiert wird. Im Folgenden soll dieser Aspekt als subjektive Sicherheit bezeichnet werden und er basiert darauf, wie die persönliche objektive Freiheit durch den Betroffenen selbst eingeschätzt wird. Wichtig ist dabei, dass subjektive Sicherheit kein endgültiger Zustand ist, sondern ein Prozess, der von einer Vielzahl von Faktoren abhängt. Frevel sieht das Sicherheitsgefühl in engem Zusammenhang mit Kriminalitätseinstellungen. Er unterscheidet fünf verschiedene Einflüsse (vgl. FREVEL 2003: 326 ff.), die auf die Entstehung und Entwicklung des Sicherheitsgefühls Einfluss nehmen. Im Sozialisationsprozess werden durch »intra- und interpersonelle Auseinandersetzung des Sozialisanden mit den Erwartungen, Werthaltungen und Verhaltensweisen der wichtigsten Bezugspersonen und weiterer Sozialisatoren« (ebd.: 326) erste Einstellungen gebildet. Eine Vielzahl von Faktoren wirkt zum Beispiel auf die Furcht vor Kriminalität:

"Liebe und Gewalt sowie verschiedene(.) Formen von Sanktionen bei abweichendem Verhalten, die Nähe bzw. Ferne von Kriminalität, die Ausbildung von Vertrauen und Misstrauen gegenüber den Bezugspersonen, die Stringenz von Erziehung und vieles mehr (...) « (ebd.).

Hier werden die Grundlagen gelegt, wie sich das subjektive Sicherheitsgefühl später manifestieren wird. Ebenfalls wichtig sind die primären Erfah- 
rungen mit Kriminalität. Nach Frevel spielen dabei der Umgang mit dem Opfer, die Aufarbeitung der Tat durch das Opfer und der Delikttyp wichtige Rollen. Je nach persönlicher Bewältigung der Tat im Zusammenspiel mit Partnern wie der Polizei kann sich die Einschätzung der persönlichen Sicherheitslage wandeln, wobei die Folgen der Viktimisierung nicht immer gleich laufen. Die Angst vor Einbrüchen nach einem solchen kann dauerhafte Angst vor weiteren erhöhen, gemäß dem Motto »das passiert doch nicht zweimal nacheinander k kann sich auch eine gewisse Sorglosigkeit ausbreiten. Auch andere Personen tragen mit ihren »Informationen« (ebd.: 327), wie Frevel es nennt, dazu bei, dass sich die subjektive Sicherheit verändern kann. Vor allem die »stellvertretenden Opfererfahrungen« (ebd.) würden hierbei eine entscheidende Bedingung sein. Diese »indirekte Viktimisierung« (ebd.) hätte einen enormen Einfluss auf die individuelle Wahrnehmung. In der Tat vergrößert sich dieser sekundäre Bereich mit jedem Bekannten, der wiederum auf seinen Bekanntenkreis zurückgreift, sprunghaft. Natürlich kann durch die Informationen anderer Leute prinzipiell auch das Sicherheitsgefühl gesteigert werden. Nur allzu oft ist aber die vorherrschende Anschauung bezüglich sicherheitsbedrohen der Kriminalität weit verbreitet. Entsprechende Vorurteile und Stereotype, die sich so etablieren können, haben ebenfalls weitreichende Auswirkungen auf die subjektive Sicherheit.

Als letzten Einfluss nennt FreVel die Massenmedien, deren Berichterstattung zur Verfestigung bestimmter Perspektiven erheblich beiträgt. Ohne dies hier empirisch prüfen zu wollen, kann man davon ausgehen, dass Berichte über bestimmte Straftaten in den Massenmedien weit verbreitet sind. Man hat das Gefühl - wie auch bei aktuellen Wirtschaftsthemen -, dass die Kriminalitätsberichterstattung mehr Raum einnimmt und ein negativeres Bild zeichnet 
als eigentlich angemessen wäre. Aus journalistischer Sicht ist das verständlich, da ein Bericht über einen Raubüberfall in einem Geschäft interessanter ist als die Schlagzeile, dass schon wieder mehrere Hundert Menschen ohne Vorkommnisse ungestört eingekauft haben. Ständige negative Berichterstattung in Nachrichtensendungen kann zu eigentümlichen politischen Reaktionen wie der des rumänischen Senats führen, der im Juni 2008 beschloss, dass »Radio und TV (künftig) in gleichem Umfang Informationen über negative und positive Ereignisse verbreiten « (SCHWARTZ 2008) müssen. Die praktische Umsetzung dieses Medienkonzeptes steht allerdings noch aus. Abseits allermöglicherweise auch unfreiwilliger - Persiflage gilt aber, dass die Einschätzung der eigenen Sicherheit von der medialen Berichterstattung erheblich beeinflusst wird.

Die Medien agieren allerdings nicht isoliert, sondern vielmehr nachfrageorientiert und befriedigen mit ihrer Kriminalitätsberichterstattung, die nach FREVEL (2003: 328) mit der »kriminellen Realität nicht viel gemein« hat, auch eine Nachfrage von Seiten ihres jeweiligen Publikums. Es ist kaum verbindlich zu klären, was hier Henne und was das Ei ist, da sich Angebot und Nachfrage gegenseitig verstärken und bedingen, so dass im Laufe dieses Prozesses oftmals der Beginn der Abläufe nicht mehr klar zu verorten ist. Die von FREVEL beschriebenen Einflüsse auf die Entstehung des Sicherheitsgefühls wirken verschränkt und laufen parallel und kontinuierlich ab. Sie alle basieren auf Primärerfahrungen oder auf der Aufnahme, Verarbeitung und der Weitergabe von Wissen. Berichte und Perspektiven anderer, egal ob sie von Freunden, Bekannten oder durch die Massenmedien erfolgen, haben einen enormen Einfluss auf das Sicherheitsempfinden aller Menschen. Je mehr der Einzelne in der Lage ist, die verschiedenen Informationen zu filtern und im jeweiligen 
Kontext zu interpretieren, nähert sich die subjektive der objektiven Sicherheit graduell an, wobei klar sein muss, dass weite zukunftsorientierte Bereiche dauerhaft unsicher bleiben. Dreht man die Perspektive um, so wird deutlich, dass der Einzelne auch auf andere einwirkt. Dies betrifft ganz besonders den Bereich der Sozialisation, wenn durch elterliche Erziehung das Sicherheitsgefühl intendiert oder unabsichtlich an die Kinder weitergegeben wird.

Sowohl objektive als auch subjektive Sicherheit sind wichtige Bestandteile einer Analyse des Begriffs Sicherheit insgesamt. Veränderungen des individuellen Sicherheitsgefühls können durch Verhaltensmodifikationen der Beteiligten auch objektiv die Sicherheitslage beeinflussen. Auch hier gilt das schon zuvor beim Aspekt Freiheit in Unterkapitel 2.1.3.3 dargestellte Prinzip der self-fullfilling prophecy nach MERTON, die auf dem oft zitierten ThomasTheorem basiert: »If men define situations as real, they are real in their consequences« (THомAs/THомAS 1928: 572). Diese als Thomas-Theorem bekannt gewordene Aussage gilt leicht abgewandelt auch in diesem Zusammenhang und zwar sowohl auf der individuellen als auch auf der gesellschaftlichen Ebene wie bei der Freiheit auch für den Aspekt Sicherheit. Die individuelle Wahrnehmung der Sicherheitssituation, also die Subjektivierung der objektiven Gegebenheiten kann die Randbedingungen drastisch verändern: Unsichere Menschen verhalten sich auch unsicher. Sie beginnen scheinbar gefährliche Orte und Menschen, die vom Äußeren her einem gewissen als unsicher betrachteten Typus gleichen, zu meiden und zeigen auch körperliche Merkmale von Angst. Durchaus denkbar ist, dass genau diese äußeren Merkmale des Unsicherheitsgefühls der erste Schritt in Richtung Viktimisierung sind, da bestimmte Täter dadurch geradezu angelockt werden. In diesem Beispiel erfüllt sich die Prophezeiung in dem Sinn, dass sich eine perzipierte Gefähr- 
dungslage in Form tatsächlicher Bedrohung manifestiert. Gleiches gilt auch in größerem Rahmen für den Bereich Freiheit. Die Furcht vor Kriminalität oder Terror, lässt politische Inhalte akzeptabel werden, die ansonsten keinerlei Durchsetzungschancen hätten. Individuelle Gefährungslagen manifestieren sich so in größeren politischen Kontexten, etwa im Rahmen von Sicherheitsgesetzen oder der Ausweitung von polizeilichen Befugnissen.

Aus der subjektiven Wahrnehmung entwickelt sich in obigen Beispiel eine reale Bedrohung. Aber auch wenn es nicht zu einer unmittelbaren Viktimisierung kommt, wirkt das persönliche Empfinden auf die objektive Sicherheit zurück auch wenn das Zusammenspiel von objektiver und subjektiver Sicherheit nicht zwangsläufig in einer Opfergeschichte enden muss. Ebenso denkbar sind Auswirkungen wie beispielsweise die Wahl des Wohnorts, die Art der Freizeitgestaltung, das Meiden bestimmter Situationen oder Orte, das politische Verhalten oder die konkrete Zustimmung beziehungsweise Ablehnung oder Akzeptanz von bestimmten sicherheitspolitischen Maßnahmen.

Die folgenden Abschnitte dieser Arbeit werden sich im Kern auf Sicherheit im objektiven Sinne stützen, nicht zuletzt weil ihre Veränderungen klarer und eindeutiger zu registrieren sind sind. Sicherheit im subjektiven Sinn wird immer dann in den Mittelpunkt treten, wenn anhand bestimmter Ereignisse oder Handlungsweisen die Motivation und die Interessen einzelner Akteure untersucht werden sollen.

\subsection{Das Verhältnis von Freiheit und Sicherheit}

Nachdem bislang Sicherheit und Freiheit isoliert voneinander behandelt wurden, sollen nun das Zusammenwirken beider Phänomene - vorerst noch 
auf einer theoretisch ausgerichteten Ebene - betrachtet werden. Es soll gezeigt werden, wie sich beide gegenseitig bedingen können und wie man bestimmte Entwicklungen möglichst optimal veranschaulichen kann.

\subsubsection{Das Zusammenwirken von Freiheit und Sicherheit - Ideengeschichte}

Im alltäglichen Gebrauch werden die Begriffe Sicherheit und Freiheit oftmals in enger Verbindung miteinander verwendet. Die Mottos »Freiheit braucht Sicherheit«, "Sicherheit und Freiheit«, »Keine Freiheit ohne Sicherheit» und auch der Titel dieser Arbeit weisen auf eine enge Verbindung zwischen beiden Begriffen hin. In der wissenschaftlichen Literatur wird mitunter Freiheit als Bestandteil von Sicherheit interpretiert. Nach SCHOLLER/Bross (1978. 209) umfasst beispielsweise die öffentliche Sicherheit die »Unversehrtheit von Leben, Gesundheit, Ehre, Freiheit und Vermögen der Bürger, sowie der Rechtsordnung und der Einrichtungen des Staates«. Auch Krollmanns Definition von öffentlicher Sicherheit beinhaltet Freiheit: „Die öffentliche Sicherheit umfaßt den Bestand des Staates und seiner Einrichtungen (...), den Schutz von Leben, Gesundheit, Freiheit und Ehre des einzelnen (...)« (KRoLLmanN 1966: 14).

Betrachten wir zunächst das Zusammenwirken von Sicherheit und Freiheit als Inhalte, die sich gegenseitig stützen und bedingen. Sicherheit tritt in dieser Konstellation als Grundlage und Basis für Freiheit auf, wobei zunächst offen bleiben kann, wie groß die tatsächliche Relation von Sicherheit und Freiheit ist. Denkbar ist sowohl ein relativ gering ausgeprägter Sicherheitssockel, auf der dann Freiheit aufsetzt (siehe Abbildung 5). Das Verhältnis ist aber auch in 


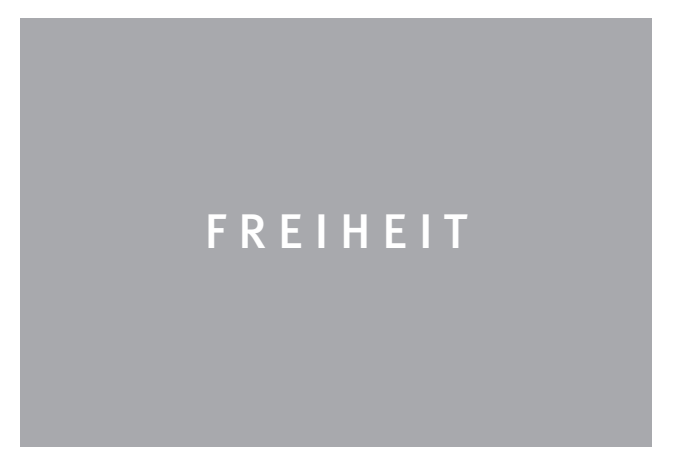

SICHERHEIT

\section{FREIHEIT}

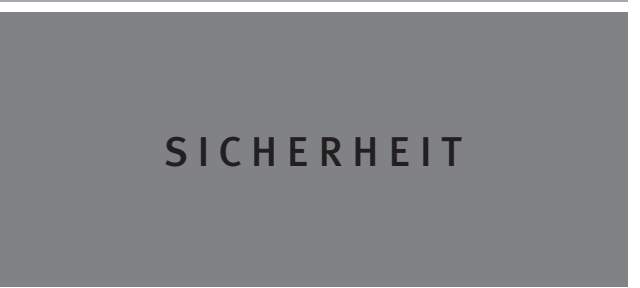

Abbildung 5 Sicherheit als Basis für Freiheit

anderer Ausprägung denkbar: Ein großer Sockel an Sicherheit wird von einem relativ geringen Anteil an Freiheit begleitet. Die unterschiedliche Höhe der beiden Säulen aus Sicherheit und Freiheit ist Absicht, die Relation der beiden steht in dieser Darstellung in keinem Zusammenhang zum abslouten Grad an Sicherheit und Freiheit in einer Gesellschaft.

Diese modellhafte Darstellung steht für zwei wichtige Gedankengänge. Zum einen soll veranschaulicht werden, dass im Modell Freiheit und Sicherheit als eigenständige Faktoren existieren, wobei unabhängig von der tatsächlichen Gewichtung Sicherheit die Grundvoraussetzung und Basis für unterschiedlich große Anteile an Freiheit ist: Hier gibt es ohne Sicherheit keine Freiheit. Im alltäglichen Gebrauch gibt es diese Koexistenz der beiden Aspekte häufiger, vor allem wenn man einzelne Teilbereiche von Sicherheit und Freiheit betrachtet. Gerade wirtschaftliche Sicherheit wird sehr häufig als Basis für libertinage (freies Handeln im Sinne von Entscheidungsfreiheit) und Freiheit im Sinne von Streben nach Erfolg genutzt. Ein großer Anteil von scheinbar freien 
Entscheidungen wird durch entsprechende Absicherungen und finanzielle Mittel erst ermöglicht. Auf verschiedenen Ebenen lassen sich dafür Beispiele finden. Die Entscheidung zwischen dem Urlaub in einem Ein- oder Fünfsternehotel ist ohne adäquaten finanziellen Hintergrund lediglich theoretischer Natur. Was auf der privaten Ebene für solche Entscheidungen zutrifft, gilt in analoger Form bei fast allen wirtschaftlichen Entscheidungen auch für Einzelpersonen, Unternehmen und auch für den Staat.

Zum anderen ist aber auch Sicherheit im Sinne von Gewissheit und Vorhersehbarkeit eine wichtige Vorbedingung für freie Entscheidungen, da dadurch Situationen erst einschätzbar und bewertbar werden.

Das oben skizzierte Modell von Freiheit, die auf einem Sockel an Sicherheit aufsetzt, ist aber nur eine mögliche Perspektive, die das komplexe Verhältnis von Sicherheit und Freiheit abzubilden versucht. Sie muss notwendigerweise ergänzt werden durch eine Darstellung, die explizit berücksichtigt, dass Freiheit und Sicherheit auch im Gegensatz zueinander stehen können.

Abbildung 6 stellt eine Möglichkeit dar, den Konflikt zwischen Sicherheit und Freiheit und die Veränderung ihrer Relation abzubilden. Die schraffierten Flächen eines Rechtecks repräsentieren dabei zusammen jeweils die gesamte maximale Quantität von Freiheit und Sicherheit in einer beliebigen Gesellschaft, die hier im Modell immer gleich bleibt. Die fünf verschiedenen Rechtecke zeigen verschiedene mögliche Gesellschaftszustände, bei denen ein Mehr an Sicherheit immer mit einem Weniger an Freiheit einher geht und vice versa. Extreme Beispiele bei denen entweder Freiheit oder Sicherheit gegen Null gehen sind nur hypothetisch denkbar: Die vollkommene Freiheit ist auf gesellschaftlicher Ebene ohne einen Anteil an Sicherheit nur schwer vorstell- 

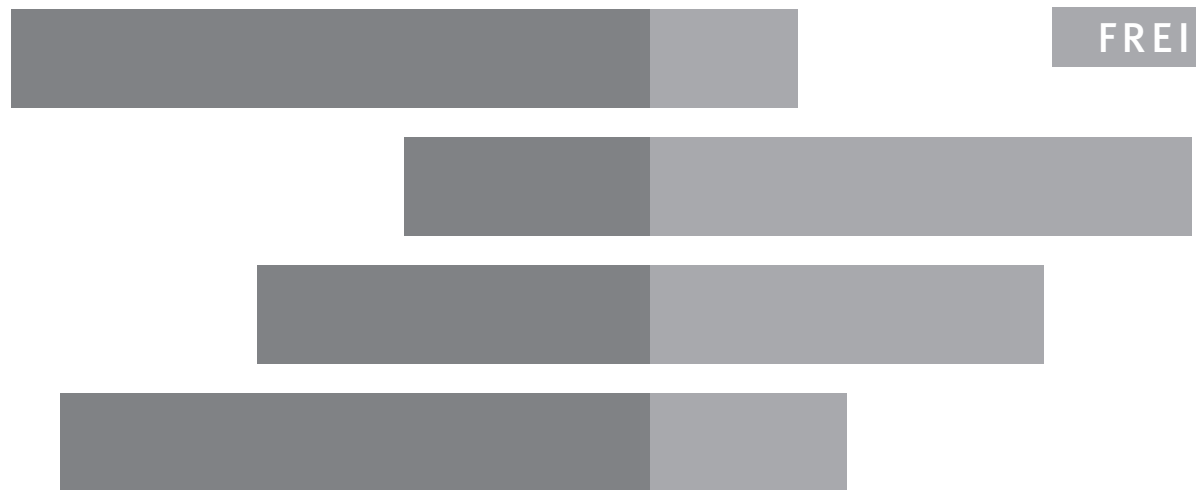

SICHERHEIT

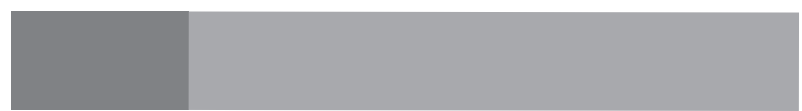

Abbildung 6 Sicherheit und Freiheit im Konflikt

bar. Ihr Gegenstück, die völlige Sicherheit ohne Freiheit, ist der Zustand in einem Kriseninterventionsraum einer psychiatrischen Einrichtung - im Volksmund auch als Gummizelle bekannt. Es fällt ebenfalls sehr schwer, sich diesen Zustand als gesellschaftliche Realität vorzustellen.

Um die oben stehenden Modelle mit Sinn und Leben zu füllen, soll an dieser Stelle der Arbeit der Blickwinkel um zwei wichtige Aspekte erweitert werden. Zum einen gilt es, näher zu betrachten, welche wichtigen Akteure im Bereich von Sicherheit und Freiheit agieren und wie sie das Verhältnis der beiden Phänomene prägen: Was sind die Ursprünge des heute existierenden Verständnisses von Freiheit und Sicherheit und wer gefährdet sie? Zum anderen soll ein kurzer Rückblick auf die ideengeschichtliche Entwicklung von Freiheit und Sicherheit die Akteursperspektive ergänzen und mit den entsprechenden theoretischen Inhalten abgleichen. 
Der zentrale Begriff, der in diesem Kontext von erheblicher Bedeutung ist, entstammt aus der politischen Theorie und vereint innerhalb der Politikwissenschaft eine ganze Reihe von Ansätzen von LOCKE über RousSEAU bis HoввES. Die folgenden Aufführungen widmen sich in diesem Sinne der so genannten Vertragstheorie, ein Begriff, der bis heute wissenschaftliche Relevanz besitzt und auch im alltäglichen Denken eine oftmals unbemerkte Resonanz gefunden hat. Wenn Autoren wie GLAESSNER davon sprechen, dass es die Aufgabe des politischen Gemeinwesens heute ist, den Bürgern die Chance zu geben, »frei und unangefochten leben zu können« (GLAESSNER 2002: 7) so stehen im Hintergrund dieser Aussage Grundgedanken der Vertragstheorie. Der Staat existiert danach also nicht ohne Ziel, sondern irgendjemand hat im Sinne der Bürger das entscheidende Ziel definiert, an dessen Umsetzung sich der Staat dann letztlich auch messen lassen muss. BALLESTREM formuliert dazu den gemeinsamen Grundgedanken der politischen Vertragstheorien:

»Eine politische Ordnung ist nur dann legitim, wenn ihr alle, die unter dieser Ordnung leben, als Freie und Gleiche in einem Vertrage zustimmen können. (...) Gerechte Herrschaft, im Gegensatz zur Gewaltherrschaft (...), beruht auf der Zustimmung freier Bürger oder auf einem Übereinkommen zwischen Volk und Herrscher« (BALLESTREM 1983: 2).

Diese Definition beinhaltet neben der Beantwortung der Frage der Legitimität und den Vorbedingungen einer politischen Ordnung auch die Akteure, die innerhalb dieser wirken. Dazu zählen die freien Bürger, die den Vertrag mit dem Herrscher als weiterem Partner abschließen. Daraus ableiten kann man die folgenden Konstellationen, in denen es zu sicherheits- oder freiheitsrelevanten Handlungen zwischen den Akteuren kommen kann: zwischen den Bürgern untereinander einerseits und zwischen Bürger und Herrscher/Staat andererseits. Stellvertretend für diese beiden Konfliktstellungen kann man 
zwei Vertragstheorien herausgreifen, welche diese Konstellationen geradezu idealtypisch behandeln. Eine klassische Vertragstheorie, die sich den Konflikten unter den Bürgern annimmt, ist die von HobBes (1578-1679), der im 17. Jahrhundert in seinem Leviathan zu diesen Konflikten Stellung nimmt. Hoвbes geht dabei von einem Naturzustand der Menschen aus, der von der Freiheit ausgeht »nach welcher ein jeder zur Erhaltung seiner selbst seine Kräfte beliebig gebrauchen und folglich alles, was dazu etwas beizutragen scheint, tun kann« (НоввES 1970: 118). Dieser Naturzustand ist aber geprägt von Konflikten zwischen den einzelnen Gesellschaftsmitgliedern, der nach HовBES durch »Mitbewerbung, Verteidigung und Ruhm« (ebd.: 115) verursacht wird. Mitbewerbung hat nach Новвеs Herrschaft zum Ziel, die letztlich zum Streit über Gewinn führt, die Verteidigung des eigenen Besitzes und der physischen Integrität beabsichtigt, Sicherheit und Wohlfahrt herzustellen, und Ruhm führt letztlich zu Konflikten bezüglich der eigenen und fremden Einschätzung von individuellen Qualitäten (vgl. ebd.). Im Naturzustand fehlen regulierende Mechanismen, welche die entstehenden Konflikte lösen. НоввEs nimmt daher an, dass auf diese Weise ein Krieg aller gegen alle (vgl. ebd.) über längere Zeit etabliert wird, in dem schlussendlich die Menschen mit dem größten Verstand und der größten körperlichen Kraft in der Lage sein würden, für sich Sicherheit zu kreieren (ebd.). Das Verlangen der Menschen nach einem bequemeren Leben und Selbsterhaltung führt nach НоввеS in eine andere Richtung, nämlich in ein Gemeinwesen, das er als »Leviathan« (ebd.: 155) bezeichnet. Dieses entsteht durch einen freiwilligen Vertrag, den die Mitglieder des Gemeinwesens untereinander schließen und in dem sie ihr Recht, sich selbst zu beherrschen, gemeinsam aufgeben und auf den Staat - darunter versteht НоввеS eine Person, die nach eigenem Gutdünken die ihm übertragene Macht 
aller "zum Frieden und zur gemeinschaftlichen Verteidigung" (ebd.: 156) anwendet - übertragen. Die höchste Gewalt steht unteilbar einem Stellvertreter dieses Staates zu, seine anderen Mitglieder sind Bürger und Untertanen. HоввES macht deutlich, wie umfassend die Befugnisse seines Leviathan sind (vgl. HоввES 1970: 157 ff.), der selbst weitgehend außerhalb der verschiedenen Konflikte seiner Bürger steht: Als staatliches Gebilde, das freiwillig durch freie Bürger (ebd.: 160) gegründet wird, steht er unantastbar über ihnen, und kann selbst keinerlei Unrecht begehen (ebd.) und er kann die höchste Gewalt auch nicht verlieren, wenn er schlechte Arbeit leistet (ebd.: 158). Die erklärten Ziele des geschlossenen Vertrages sind »Frieden und Schutz« (ebd.: 160), »damit die Bürger im Inneren und von außen her in Sicherheit leben (...)« (ebd.: 160 f.) können. Um dieses Ziel zu erreichen, billigt НоввеS dem Staat ein umfassendes Paket an Kompetenzen zu: Teil der höchsten Gewalt ist das Richteramt, das Streitigkeiten im Inneren zwischen den Bürgern schlichtet. Nach Außen hin sichert der Staat seine Bürger durch sein militärisches Potenzial ab. Er besitzt außerdem dauerhaft die Möglichkeit, »Krieg gegen andere Staaten nach Gutdünken« (ebd.: 162) zu führen, solange dieser als vorteilhaft angesehen wird:

„Denn der Schutz der Bürger hängt von den Kriegsheeren ab, die Stärke dieser von der Einigkeit des Staates und diese allein von der Person des Oberherrn. Das Recht über die Kriegsheere ist schon an und für sich die höchste Gewalt, weil darin die ganze Stärke des Staates besteht (..)« (ebd.).

Der Einfluss des Leviathans reicht noch weiter und bezieht auch die Kompetenz zu entscheiden mit ein,

»was zur Erhaltung oder zur Störung des Friedens dienen kann; folglich auch zu bestimmen, zu welcher Zeit, unter welchen Bedingungen und wem es erlaubt sei, das Volk aufzuklären; welche Bücher verboten werden müssen und wer darüber die Aufsicht 
führen soll. Handlungen haben ihren Grund in Meinungen; folglich müssen diese unter Aufsicht genommen werden, wenn man Frieden und Einigkeit in einem Staat erhalten soll« (ebd.: 161).

Im Mittelpunkt der Vertragstheorie von Новвes stehen also die Konflikte der Bürger untereinander, welche sie in der umfassenden Machtübertragung auf den Staat zu lösen versuchen.

Die Vertragstheorie von JoHn LOCKE (1632-1704) erweitert diese Konstellation, indem die Ebene des Staates mit in die Konfliktstellung einbezogen wird. In seinem Second Treatise of Government will LOCKE zeigen, »wie Entstehung, Zweck und Institutionen eines Regierungssystems zu denken seien, die von freien Menschen als legitim anerkannt werden« (EUCHNER 2001: 19). Ausgangspunkt seiner Überlegungen ist dabei ebenfalls ein Naturzustand, aber bei Locke findet sich im Gegensatz zu Новвеs kein Krieg aller gegen alle, der diesen Naturzustand dominiert. Das Menschenbild LocKES geht vielmehr davon aus, dass die Menschen von Natur aus in einem »Zustand vollkommener Freiheit« (LOCKE 1974: 5) zusammenleben, der allerdings mit einigen Unsicherheiten behaftet ist. Da der Einzelne im Naturzustand seine Freiheit gegenüber anderen selbst verteidigen muss, droht immer auch der Kriegszustand, wenn ein anderer die Freiheit - neben dem Leben zählt bei LOCKE dazu auch das Eigentum an materiellen Dingen, soweit es durch Arbeit (vgl. ebd.: 32) erworben wurde - bedroht. Die Menschen begegnen diesem Zustand dadurch, dass sie "politische oder bürgerliche Gesellschaften« (ebd.: 67) gründen, in denen der Einzelne auf sein Recht auf Vollstreckung des Naturgesetzes verzichtet und dieses auf das Gemeinwesen überträgt. Da die größte Gefahr, die dem neu gegründeten Gemeinwesen droht, der Missbrauch der übertragenen Macht (vgl. EUCHNER 2001: 23) ist, schlägt LoCKE 
eine Teilung in die legislative, exekutive und föderative Gewalt (vgl. LoCKE 1974: 111 ff.) vor. Diese Teilung der Gewalten wird von MONTESQUIEU in seinem Werk »De l'esprit de loi« ein Jahrhundert später aufgegriffen und in die bis heutige gängige und vertraute Form gebracht:

»Wenn in derselben Person oder der gleichen obrigkeitlichen Körperschaft die gesetzgebende Gewalt mit der vollziehenden vereinigt ist, gibt es keine Freiheit; denn es steht zu befürchten, daß derselbe Monarch oder derselbe Senat tyrannische Gesetze macht, um sie tyrannisch zu vollziehen. Es gibt ferner keine Freiheit, wenn die richterliche Gewalt nicht von der gesetzgebenden und vollziehenden getrennt ist. Ist sie mit der gesetzgebenden Gewalt verbunden, so wäre die Macht über Leben und Freiheit der Bürger willkürlich, wenn der Richter Gesetzgeber wäre. Wäre sie mit der vollziehenden Gewalt verknüpft, so würde der Richter die Macht eines Unterdrückers haben. Alles wäre verloren, wenn derselbe Mensch oder die gleiche Körperschaft der Großen, des Adels oder des Volkes diese drei Gewalten ausüben würde (...)« (MONTESQUIEU 1992: 215).

Judikative, Legislative und Exekutive teilen sich demnach die Staatsgewalt auf und kontrollieren sich gegenseitig. Fasst man die beiden Ansätze zusammen, so kann man den Zusammenhang zwischen Freiheit und Sicherheit auf der ideengeschichtlichen Ebene leicht nachvollziehen. Der Staat des HoBbES'SCHEN Leviathan wird zum mächtigen Schlichter und Beherrscher von konfliktgeplagten Einzelmenschen: Er garantiert letztlich die Ordnung und die Sicherheit der einzelnen Bürger. Die Erreichung dieser Ziele ist die Legitimation für die »Konzentration von Macht und die Monopolisierung von Gewalt in den Händen des Staates« (GLAeSSNer 2002: 7). Bei Locke und Montesquieu bekommt der Staat ebenfalls Macht übertragen, nur wird diese zur Vermeidung von Missbrauch »eingehegt« (ebd.) und kontrolliert. Der Einzelne soll dadurch nicht nur Sicherheit vor den Mitmenschen erfahren, sondern auch in Sicherheit vor Übergriffen durch die Staatsgewalt sein. Neben der Konfliktsituation, die einzelne Menschen betrifft, wird hier eine neue zwischen 
Bürgern und dem Staat aufgezeigt. Das dritte Kapitel dieser Arbeit wird im Verlauf noch ausführlich darauf eingehen, wie heute versucht wird, durch bestimmte politische Strukturen und Institutionen diese Konflikte zu beherrschen.

Politische Denker wie Hobbes, Locke, Rousseau, Kant, Hegel, Mill oder RAWLS haben bis heute großen Einfluss auf die Art und Weise wie das Verhältnis Bürger und Staat wahrgenommen wird. Heutige Vorstellungen von Freiheit und Sicherheit existieren nicht aus sich selbst heraus gewissermaßen im luftleeren theoretischen Raum, sondern in Denktraditionen, die alle auf bestimmten anthropologischen Grundannahmen beruhen. Nicht immer kommt den einzelnen Menschen dabei eine aktive Rolle zu, wie etwa bei der klassischen Vertragstheorie. Vielmehr finden sich mitunter auch Ansätze, welche die Machtübertragung an andere nicht als Transfer von freien Menschen zur Etablierung von Legitimität für ein politisches System interpretieren, sondern vielmehr von der Annahme ausgehen, Menschen würden gezielt Freiheit vermeiden:

»Die Mehrzahl der Menschen scheint es vorzuziehen, daß andere die meisten Entscheidungen für sie treffen, und praktisch alle Menschen wollen, daß ihnen eigene Entscheidungen von anderen abgenommen werden. Das gilt aber nicht nur für Entscheidungen, sondern auch für Handlungen, Meinungen usw. Diese Neigung der Menschen, das mögliche Maximum an Freiheit zu vermeiden, ist nicht unbedingt beklagenswert. Sie hängt zusammen mit dem Wunsch des Menschen, möglichst bequem zu leben und seine lebenswichtigsten Aufgaben auf dem Weg des geringsten Widerstandes zu verwirklichen (...)« (FRIEDRICH 1970: 229).

Unter Berücksichtigung der obigen Denktraditionen kann man das besondere Verhältnis von Freiheit und Sicherheit in einer politischen Ordnung nochmal neu betrachten (siehe Abbildung 7). Freiheit und Sicherheit sind dabei 

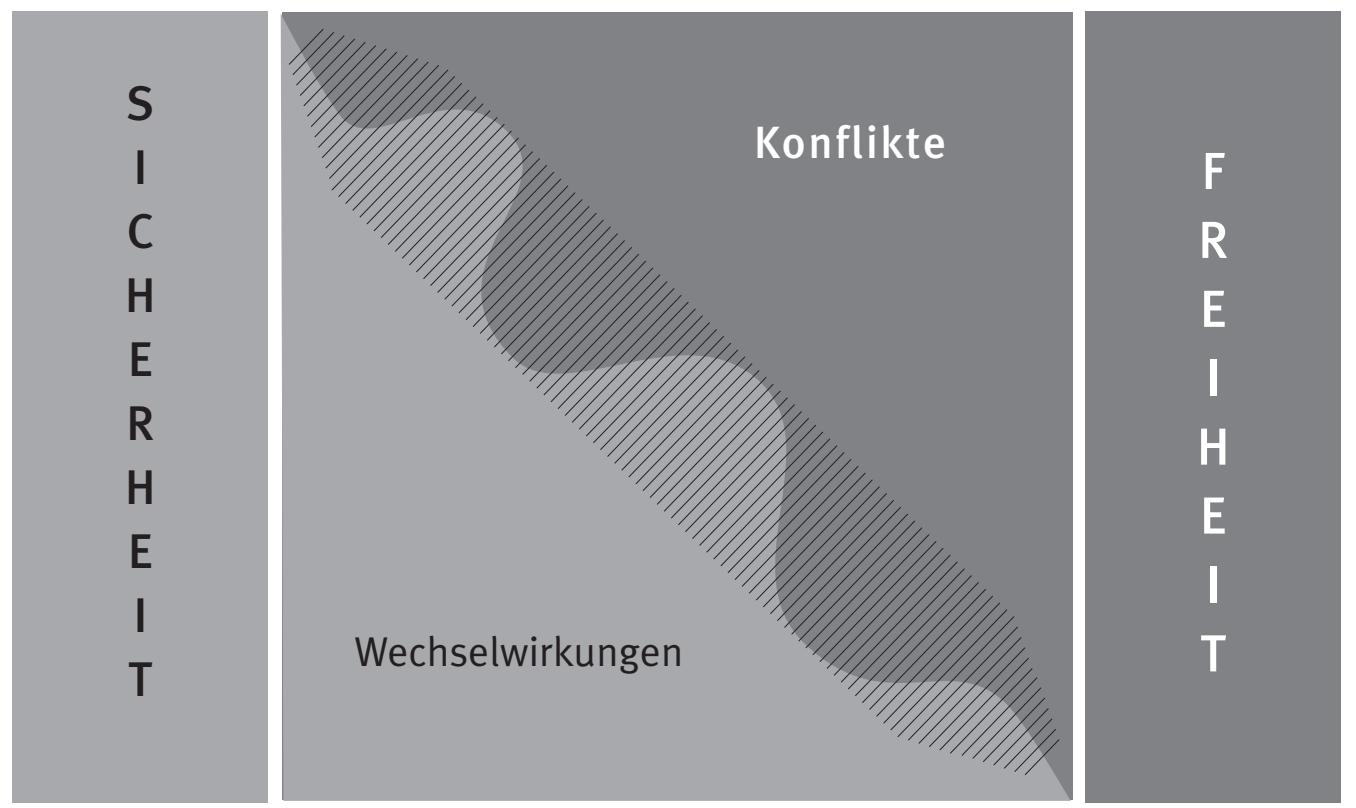

Abbildung 7 Sicherheit und Freiheit

wichtige Säulen, die jeweils für ein gewisses Maß an grundlegender Freiheit in jeder Gesellschaft stehen. Für Sicherheit in ihren verschiedenen Bedeutungen und vor allem auch in Form von Konfliktregulierung sorgt der Staat und in kleinerem Umfang auch der Bürger. Dem gegenüber steht als zweite Säule die Freiheit, die auf den Entscheidungen der gesellschaftlichen Akteure gründet. Wichtigstes Element ist dabei die Eigenkontrolle über die persönlichen Distanzen zu den anderen Akteuren und das Wissen um reglementierende Rahmenbedingungen. Zwischen beiden Aspekten kommt es aber auch zu Konfliktstellungen, vor allem, wenn der Staat als Konfliktschlichter für Sicherheit einsteht und Freiheiten der Individuen beispielsweise durch Freiheitsentzug beschneidet. Der mittlere Teil der Abbildung repräsentiert die Verschiebungen zwischen Freiheit und Sicherheit in einer Gesellschaft, allerdings unter der 
Berücksichtigung von Veränderungsprozessen. Interessant ist dabei nicht so sehr ein bestimmter Zeitpunkt, an dem man ein bestimmtes Maß an Freiheit und Sicherheit in einer Gesellschaft feststellen kann, sondern die Suche nach Verlagerungen und Veränderungen zwischen Freiheit und Sicherheit, die sich anhand konkreter Ereignisabfolgen dokumentieren lassen. Die Schraffur der Zone zwischen Freiheit und Sicherheit soll verdeutlichen, dass es dabei auch Grauzonen gibt, die den klaren analytischen Blick trüben. Nicht immer kann bestimmten Ereignissen eine klare Wirkung zugeordnet werden, zuweilen verändern sich Freiheit und Sicherheit gemeinsam aber nicht im Sinne eines Nullsummenspiels. Es wird Aufgabe dieser Arbeit sein, sowohl die Details der einzelnen Entwicklungen als auch deren große, richtungsweisende Auswirkungen gemeinsam und angemessen zu erfassen. Der Idealzustand von Freiheit und Sicherheit, den MonTESQUIEU seinen ausführlichen Gedankengängen zur Gewaltenteilung in einem Staat voran stellt, setzt Vertrauen und Ruhe als wichtige Inhalte mit Sicherheit und Freiheit in Bezug: »Die politische Freiheit des Bürgers ist jene Ruhe des Gemüts, die aus dem Vertrauen erwächst, daß ein jeder zu seiner Sicherheit hat. Damit man diese Freiheit hat, muß die Regierung so eingerichtet sein, daß ein Bürger den anderen nicht zu fürchten hat“ (MONTESQUIEU 1992: 215). Der Verlauf der Analyse wird zeigen, ob dieser Zustand überhaupt zu erreichen ist und wenn ja, ob man sich auf dieses Ideal hin oder davon weg bewegt.

\subsubsection{Privatheit als Schnittstelle von Freiheit und Sicherheit}

Aktuelle Diskussionen über Freiheit und Sicherheit in modernen Gesellschaften bemühen schon fast unvermeidlich Inhalte aus GEORGE ORWELLS Roman 
"1984«, der das Leben in einer dystopischen Gesellschaft schildert, in der die Bürger in der Öffentlichkeit und bis in die eigenen vier Wände kontinuierlich audiovisuell überwacht und kontrolliert werden und sämtliche Privatheit vollkommen obsolet und beseitigt wird: Über den sogenannten Televisor werden die Charaktere des Romans dauerhaft überwacht ${ }^{1}$, lediglich höhere Parteikader können die Überwachungsgeräte ein halbe Stunde am Tag abschalten. Zudem müssen sie ernste psychische und physische Folgen befürchten, wenn sie versuchen, aus dem System auszubrechen oder sich zu widersetzen. Die Figur des Big Brother, der sinnbildlich für diese umfassende Fremdkontrolle steht, hat aus diesem Roman den Weg in unsere Alltagssprache gefunden. Die Verbindung von Privatheit und Freiheit ist aber keinesfalls ausschließlich eine Frage der Literatur. Auch die Allgemeine Erklärung der Menschenrechte aus dem Jahre 1948 erwähnt die Privatheit im Artikel 12:

»Niemand darf willkürlichen Eingriffen in sein Privatleben, seine Familie, seine Wohnung und seinen Schriftverkehr oder Beeinträchtigungen seiner Ehre und seines Rufes ausgesetzt werden. Jeder hat Anspruch auf rechtlichen Schutz gegen solche Eingriffe oder Beeinträchtigungen.«

Nach GARFINKEL ist Privatheit aber nicht nur ein wichtiger Bestandteil der Menschenrechte und der individuellen Freiheit, sondern auch ein Gradmesser

1 Gerade jüngere Leser assoziieren mittlerweile mit dem Ausdruck Big Brother eher die gleichnamige Fernsehshow, wobei allerdings enorme Differenzen zur literarischen Referenz, die sich auf eine ganze Weltgesellschaft bezieht, vorzufinden sind. Die komplexen Inhalte des Romans sind erstens stark abgeändert und zweitens in wesentlich einfacherem Rahmen auf Wohngemeinschafts-Niveau abgebildet werden. Nicht zu Unrecht hat WEICHERT diese Assoziation als Ausdruck eines fragwürdigen Bildungsniveaus bezeichnet (vgl. WEICHERT 2004: 129). 
für die »power of the individual« (GARFINKEL 2001: 5), dem das Potenzial der Fremdkontrolle sowohl durch andere Individuen, Organisationen oder dem Staat gegenüber steht. Diese Privatheit ist allerdings nicht starr und festgelegt für alle Zeiten. Man kann sie stattdessen als eine »soziale und kulturell überformte Kategorie« (LAMNEK 2003: 45) bezeichnen, die unterschiedlich gestaltet sein kann und sich kontinuierlich wandelt. Ein gutes Beispiel für einen solchen Wandel findet man bei OHLER, der darauf verweist, dass es in Mitteleuropa im Mittelalter üblich war, auf Reisen in Gasthäusern mit Fremden gemeinsam in einem Bett zu übernachten, um von der Körperwärme der Bettgenossen profitieren zu können (vgl. OHLER 1986: 130). Heute sind die sozialen Distanzen weitaus größer und man würde befremdlich auf eine solche Situation in einem Gasthaus reagieren.

Privatheit ist als Begriff aber heute weiter gefasst und geht über reine Körperkontakte weit hinaus. Sieht man die einschlägige Literatur durch, so stößt man des Öfteren (u.a. bei RöSSLER 2001 oder LAMNEK 2003) auf die Definition von WESTIN, der dem Begriff privacy die Aspekte »solitude, intimacy, anonymity and reserve« (WESTIN 1967: 31) zuordnet ${ }^{1}$. Unter Ersterem versteht WESTIN das Alleinsein, das er als vollkommensten Zustand bezeichnet, den ein Mensch erreichen kann und das, obwohl die physische Verfassung des Einzelnen und zudem physikalische Einflüsse wie Hitze, Lärm oder Kälte auf ihn einwirken. Mit dem Aspekt der Intimität wird der Bezugsrahmen auf mehrere Personen erweitert. Zwischen zwei oder mehreren Menschen (Kern-

1 Eine umfangreiche Darstellung des Privatheitbegriffs mit einer ausführlichen Einordnung verschiedener theoretischer Ansätze findet sich u. a. bei RöSSLER (2001: 20ff.) und GRÄF 1993: $16 \mathrm{ff}$. 
familie, Freunde) soll sich die Interaktion frei und entspannt entwickeln können. Im Bereich der Anonymität berührt die Privatheit die Sphäre der Öffentlichkeit. Abseits aller Identifizierung und Überwachung soll sich der Einzelne frei bewegen können: »In this state the individual is able to merge into the (.) situational landscape (.). Knowledge or fear that one is under systematic observation in puplic places destroys the sense of relaxation and freedom that men seek in open spaces and public arenas."

Unter Reserviertheit versteht WESTIN letztlich den Aufbau von psychologischen Barrieren zur Aufrechterhaltung von mentalen Distanzen zur Umgebung. Die Art und Weise, wie Menschen Reserviertheit praktizieren und von anderen einfordern und die Akzeptanz durch diese anderen variiert von Kultur zu Kultur und ist ein zentraler Bestandteil von Privatheit. RösSLER erweitert die Definition von WESTIN und arbeitet das Element der Kontrolle weiter heraus:

"Als privat gilt etwas dann, wenn man selbst den Zugang zu diesem ıetwas kontrollieren kann (..). Umgekehrt bedeutet der Schutz von Privatheit dann einen Schutz von unerwünschtem Zutritt anderer. `Zugang` oder `Zutrittı kann hier sowohl die direkte, konkret-physische Bedeutung haben, so etwa, wenn ich beanspruche, den Zugang zu meiner Wohnung selbst kontrollieren zu können; es kann jedoch auch metaphorisch gemeint sein: in dem Sinn, dass ich Kontrolle darüber habe, wer welchen >Wissenszugangı zu mir hat, wer welche (relevanten) Daten über mich weiß; und in dem Sinn, dass ich Kontrolle darüber habe, welche Personen ıZugang` oder `Zutritt` in Form von Mitsprache- oder Eingriffsmöglichkeiten haben bei Entscheidungen, die für mich relevant sind« (RöSSLER 2001: 24).

Die Kontrolle, von der RöSSLER hier spricht, kann als Eigenkontrolle bezeichnet werden und sie bezieht sich sowohl auf den physischen Kernbereich des privaten Lebens als auch im übertragenen Sinn auf die Sphäre der Informationen und des Wissens bezüglich des Umfangs und der Verfügbarkeit persönlicher Daten für andere. Die ungeheuren Mengen an Daten, die von 
jedem Einzelnen existierten und die besondere Problematik von verdeckten Ermittlungsmethoden wie der z.B. der Rasterfahndung, der akustischen Wohnraumüberwachung oder der Telekommunikationsüberwachung, von denen der Einzelne unmittelbar keinerlei Kenntnis hat, erschweren die aktive Ausübung dieser Kontrolle oder machen sie mitunter unmöglich. Die Bedrohung der Bereiche, die der Einzelne kontrollieren muss, um frei zu sein, ist eine ständige Bedrohung, die ganz real und keineswegs nur latent vorhanden ist.

Letztlich verbleiben nach WESTIN drei Formen von Privatheit, die sich klar unterscheiden lassen: dezisionale Privatheit (Anspruch auf Selbstbestimmung im Gegensatz zur Fremdbestimmung), informationelle Privatheit (Schutz vor unberechtigtem Zugriff auf relevante Daten) und lokale Privatheit (tatsächlicher Schutz der eigenen Räumlichkeiten, physische Integrität) (vgl. ebd.). Der Übergang von Privatheit hin zur Öffentlichkeit ist fließend und wandelt sich im Laufe der Zeit. Der Aufbau und die funktionale Struktur der eigenen vier Wände repräsentiert eine zentrale Schnittstelle zwischen Privatheit und Öffentlichkeit. An ihrem funktionalen Wandel kann man auch den Wandel der Privatheit nachvollziehen. Bestimmte Wohnräume verändern die Funktion oder entstehen neu. Die »Intimität (.) des Wohnzimmers« (HABERMAS 1976: 63), und die "Öffentlichkeit des Salons« (ebd.) stehen für den Wandel eines solchen Raumes, wobei auch neue Schnittstellen entstehen. Heute wird beispielsweise der Computerarbeitsplatz in den Wohnungen zur Schnittstelle der weltumspannende Telekommunikationskanäle, über die der Emailverkehr, das Online-Banking und teilweise auch die Einkäufe über das Internet abgewickelt werden können.

Der Zusammenhang zwischen Sicherheit und Privatheit ist kein unmittelbarer, sondern orientiert sich mehr indirekt als Motiv und Begründung für 
Eingriffe in die Privatsphäre der Individuen. Privatheit im Sinne von Isolation und Rückzug entzieht den Einzelnen den Mechanismen der sozialen Kontrolle, die durch andere Mitglieder der Gesellschaft ausgeübt werden. Problematisch ist dabei, dass bestimmte Formen des abweichenden Verhaltens gewissermaßen im Verborgenen ausgeübt werden. Aus genau diesem Grunde ist es für den Bereich des kriminellen Verhaltens für die zuständigen Behörden zuweilen von Interesse, Einblicke in den privaten Bereich zu erhalten. Der dahinter stehende Anspruch ist, auf diese Weise für mehr (innere) Sicherheit sorgen zu können. Dieses Totschlagargument, das allen entgegen gehalten wird, die sich gegen solche Eingriffe verwahren, findet sich bei GARFINKEL, reduziert auf die griffige Formel: „Why should I worry about my privacy? I have nothing to hide« (GARFINKEL 2001: 3). Unklar bleibt letztlich auch, ob durch Eingriffe in die Freiheit und Privatheit wirklich ein Mehr an Sicherheit geschaffen werden kann oder ob dieser Anspruch ein bloßes Ziel oder gar eine bloße Rechtfertigung für eine solche Vorgehensweise darstellt. Über allem stehen die Gefahr des Missbrauchs und des Mangels an Kontrolle und Information.

\subsubsection{Zusammenfassung und zentrale Fragestellungen}

Zum Abschluss dieses umfangreichen Kapitels, das diese Arbeit auf ihr theoretisches Fundament stellt, erscheint es angemessen, in der gebotenen Kürze nochmals kurz die wichtigsten Inhalte und die entscheidenden Fragen und Thesen in übersichtlicher Form zu präsentieren:

Freiheit und Kontrolle. Der für diese Arbeit relevante Freiheitsbegriff wird bestimmt durch die Form der Kontrolle, die auf Menschen und Gruppen von Menschen in einer Gesellschaft einwirkt. Ein erhöhtes Maß an Eigenkontrolle 
steht für einen hohen Grad an Freiheit, während ein Anstieg der Fremdkontrolle für ein Absinken des Freiheitsniveaus steht.

Sicherheit. Sicherheit kann man in verschiedene Dimensionen aufschlüsseln, wobei hier vor allem der Bereich der politischen und der inneren Sicherheit von Bedeutung ist. Gerade im Sinne von letzterer wird versucht, Sicherheit durch Kontrolle herzustellen, um Gefahren durch kriminelles, beziehungsweise abweichendes Verhalten abzuwenden. Dadurch wird aus der Perspektive der inneren Sicherheit das Streben nach Konformität gleichgesetzt mit dem Streben nach Sicherheit. Als soziale Kontrolle wird der kontinuierlich ablaufende Prozess bezeichnet, der durch eine Vielzahl an Mechanismen die Konformität herstellen soll.

Verschränkung der Begriffe: Privatheit. Eingriffe in die Privatheit sind Eingriffe in die Freiheit. Die Kontrolle über die Privatsphäre und damit die Kontrolle über soziale Distanzen sind Gradmesser, wie sich die Freiheit in einer Gesellschaft verändert. Mehr Sicherheit ist oftmals das Ziel von Eingriffen in die Privatheit der Menschen, wobei offen bleibt, ob wirklich effektiv mehr Sicherheit erreicht wird oder ob Sicherheit nur das erklärte Ziel und die (gute) Absicht war.

Information und Wissen. Beides sind zentrale Bestandteile sowohl von Sicherheit als auch von Freiheit. Wir benötigen verschiedene Daten, um Eigenkontrolle ausüben zu können und damit die Freiheit abzusichern. Auch Sicherheit benötigt Wissen und Informationen, um einschätzen zu können, welche Mittel zur ihrer Erreichung nötig sind.

\section{Wichtige Fragestellungen}

- Wie haben sich bis heute Sicherheit und Freiheit in Deutschland entwickelt?

- Haben wir heute ein Mehr an Sicherheit statt Freiheit? 
- Klassische politikwissenschaftliche Denktraditionen gehen von einer Konfliktstellung Bürger-Bürger-Staat aus, die in einer bürgerlichen Gesellschaft zusammenwirken. Hier soll darüber hinaus untersucht werden, welche Akteure (Personen, Interessengruppen, Parteien, Medien, Verfassungsorgane) usw. genau bei der Entwicklung von Sicherheit und Freiheit mitwirken.

- Von besonderem Interesse sind politische und rechtliche Entwicklungen.

- Welche weiteren Entwicklungen sind in der Zukunft zu erwarten? 


\section{Chronologie des Wandels von Sicherheit und Freiheit}

Das folgende Kapitel untersucht die wichtigsten Meilensteine der Entwicklung von Freiheit und Sicherheit, die als Einschnitte oder Zäsuren bezeichnet werden können, da sie für Sicherheit und Freiheit substanzielle Änderungen bedeuteten und auch Einfluss auf praktisch alle Bereiche der Gesellschaft hatten. Ihre Darstellung folgt einem weitgehend chronologischen Aufbau, die einzelnen Unterkapitel entsprechen bestimmten zeitgeschichtlichen Ereignissen, denen wiederum wichtige Veränderungen zugeordnet werden. Wenn es der logische Aufbau erfordert, werden allerdings zeitlich auseinander liegende Ereignisse in einem gemeinsamen Abschnitt behandelt. Dies soll Redundanzen verhindern und ist der Versuch zu vermeiden, dass gedanklich Zusammenhängendes durch eine diachronische Darstellung künstlich getrennt wird. $\mathrm{Zu}$ jedem Meilenstein findet sich in jedem Abschnitt eine Bewertung, wie sich das jeweilige Ereignis auf Freiheit und Sicherheit auswirkt. Am Ende dieses Kapitels findet sich dann eine kurze Übersicht der Ereignisse, die dann wieder die zeitliche Abfolge im Einzelnen berücksichtigt.

Es sei hier darauf verwiesen, dass in diesem Kapitel nur ein kompakter Überblick über den gesamten Zeitrahmen der Bundesrepublik Deutschland gegeben wird, wobei bewusst kein Anspruch auf Vollständigkeit erhoben wird. Informierte Leser werden Einiges vermissen, da die Inhalte dieses Kapitels naturgemäß selektiert wurden. Diese Auswahl ist aber nicht beliebig erfolgt, sondern sie berücksichtigt wichtige Ereignisse, die einen Großteil der Bevölkerung betreffen und weit reichende Konsequenzen haben. Viele detaillierte Einzelaspekte werden im Folgenden bei der näheren Interpretation der Entwicklungen noch angefügt, um einzelne Sachverhalte weiter zu illustrieren. 


\subsection{Die Ausgangslage: Der freiheitliche Verfassungsstaat Bun- desrepublik Deutschland}

»Denn eine Verfassung ist kein bloß Gemachtes: sie ist die Arbeit von Jahrhunderten, die Idee und das Bewußtsein des Vernünftigen, inwieweit es in einem Volk entwickelt ist« (HEGEL 1981: 318).

Zu Beginn dieser Chronologie soll ein Blick auf die Grundstruktur und die Anfangszeit der Bundesrepublik Deutschland geworfen werden. Im Mittelpunkt des Interesses stehen das Grundgesetz der Bundesrepublik Deutschland, die verschiedenen politischen Akteure und Strukturen. Verfassungsgeschichtlich kann man trotz der deutlichen Zäsur durch das Kriegsende und der Zerschlagung der nationalsozialistischen Herrschaft nicht von einer wirklichen verfassungsrechtlichen Stunde Null sprechen, da - unter dem Einfluss der Alliierten - an voran gegangene Verfassungen angeknüpft wurde. Bei der Konzeption des Grundgesetzes wurde versucht, den Schwächen gerade der Weimarer Verfassung zu begegnen, auf den gemachten Erfahrungen aufzubauen und entsprechende Konsequenzen zu ziehen.

\subsubsection{Der Verfassungsstaat}

Der Verabschiedung des Grundgesetzes im Jahre 1949 gingen zwei wichtige politische Entwicklungen voraus, die einigen Einfluss auf die Gründung der Bundesrepublik Deutschland hatten. Dazu zählen einerseits die zuvor erfolgten Gründungen der Bundesländer, die von den Alliierten zunächst als erste politische Strukturen oberhalb der Gemeindeebene akzeptiert wurden und zu den zunächst »wichtigsten politischen Einheiten« (SONTHEIMER/BLEEK 
2000: 24) wurden. Andererseits waren auch die Beratungen des Parlamentarischen Rates auf der Herreninsel im Chiemsee von erheblicher politischer Bedeutung, da durch die dortigen Sachverständigen, die auf Initiative der Ministerpräsidenten der elf Bundesländer tagten, die wichtigsten Elemente des Grundgesetzes formuliert werden sollten. Das so entstehende »Provisorium Grundgesetz« (GÖRTEMAKER 2002: 43), das zunächst nur für die elf westlichen Bundesländer Gültigkeit hatte, entwickelte sich zur stabilen Verfassung, die mit dem Beitritt der Deutschen Demokratischen Republik seit 1990 für alle Deutschen verbindlich (vgl. HESSE/ELLWEIN 1997) ist. Das wesentliche Merkmal der neu gegründeten Bundesrepublik war damit ihre Konzeption als Verfassungsstaat. Darunter versteht man grundsätzlich einen Staat, in dem die »Grundordnung der staatlichen Herrschaft« (SUTOR 1994: 105) über ein in der Regel schriftlich fixiertes Dokument festgelegt ist. In diesem Sinne legt die Verfassung die Gesamtstruktur der politischen Organisation fest: Verfassungsorgane wie Bundespräsident, Bundesregierung, Bundestag oder Bundesverfassungsgericht und ihr Zusammenwirken werden durch verschiedene Grundgesetzartikel festgelegt.

In wertneutralem oder formalem Sinn kann man prinzipiell jede Grundordnung von staatlicher Herrschaft über ein solches Dokument als Verfassungsstaat definieren (ebd.: 105). Folgt man den Überlegungen KRIELES so hat diese Verfassung weitreichende Auswirkungen auf das politische System. Er geht davon aus, dass es in einem Verfassungsstaat keinen dauerhaft aktiven Souverän geben kann, der uneingeschränkten Zugriff auf die Politik hat (KRIELE 1981: 275), sondern dass auch der Souverän an die Verfassungsvorgaben gebunden ist. Im Grundgesetz ist dieser Sachverhalt über den Artikel 20 (2) geregelt, der alle Staatsgewalt als vom Volke ausgehend 
definiert und die Ausübung dem Volk lediglich in Form von Wahlen und Abstimmungen und ansonsten den besonderen Organen der Legislative, Exekutive und Judikative zuordnet. Die gleiche Bindung an die Verfassung trifft aber auch die Letztgenannten, die ebenfalls nur den Bestimmungen der Verfassung gemäß handeln können. Man kann mit STERnBERGER (1982:14) zu Recht behaupten, ein solcher Verfassungsstaat sei ein kompliziertes Gebilde, aber dank der Freiheit der Information auch durchsichtig. Um die politische Ordnung der Bundesrepublik in eben dieser Komplexität angemessen begreifen zu können, muss man in diesem Sinne über den rein formalen Begriff des Verfassungsstaates hinausgehen und einen »materialen, auf bestimmte Werte bezogenen Begriff der Verfassung« (SUTOR 1994: 106) berücksichtigen.

\subsubsection{Freiheit im Grundgesetz}

Heutige Verfassungen sind in der Regel das Endprodukt einer langandauernden Entwicklung - gut nachvollziehbar in England, Frankreich aber auch in Deutschland -, bei der absoluten Herrschern Macht und Einfluss abgetrotzt wurde. Im Sinne dieser »konstitutionellen Bewegung« (ebd.) ging es konkret darum, »Macht einzuschränken und zu kontrollieren, Freiheitsrechte der Bürger zu sichern (und) (.) Teilhabe- und Mitwirkungsrechte durchzusetzen« (ebd.). Die Verfassung in diesem Sinne stellt die »höchstrangige normative Grundordnung des Staates dar, in die politisch existenzielle und bestimmte traditionelle Bestandteile aufgenommen sind « (STERN 1984: 107) und die die folgenden 7 Funktionen (siehe Abbildung 8) wahrnehmen soll, wobei sich die einzelnen Funktionen auch überschneiden. Die Ordnungsfunktion des Grundgesetzes nimmt das Grundgesetz insofern wahr, als es den verfassungsmäßigen Rahmen für die Verfassungsorgane bildet und die wichtigsten Einrichtun- 
Die Verfassung soll ...

... eine Ordnungsfunktion erfüllen.

... eine Stabilisierungsfunktion wahrnehmen.

... einheitsstiftend (integrierend) wirken.

... Macht begrenzen und kontrollieren.

... Freiheit, Selbstbestimmung und Rechtsschutz des Individuums gewährleisten.

... die grundlegende Organsationsstruktur des Staates festlegen.

... Leitgrundsätze über die materialen Staatsziele und die Rechtsstellung des Bürgers im und zum Staate enthalten.

Abbildung 8 Sieben Funktionen der Verfassung des modernen Staates (vgl. STERN 1984: 82, leicht abgeändert)

gen von Exekutive, Legislative und Judikative definiert und innen klare Kompetenzen zuordnet. Eng verbunden mit der Ordnungsfunktion ist die Stabilisierungsfunktion einer Verfassung, die sich beim Grundgesetz beispielsweise in Form hoher Hürden äußert, was die Möglichkeiten zur Verfassungsänderung angeht. Die Artikel 1 und 20 sind im Zusammenwirken mit Artikel 79 (3) völlig vor Änderungen geschützt, für die anderen Grundgesetzartikel ist eine Zweidrittelmehrheit in beiden Kammern vonnöten. Die Verfassung wird damit zu einem statischen Element, dass den Fluktuationen des politischen Alltagsgeschäftes mit seinen häufigen Gesetzesänderungen weitgehend entzogen ist.

Machtkontrolle und -begrenzung wird ebenfalls durch die schon angesprochenen Aspekte erreicht und natürlich auch durch einen umfangreichen Katalog an Individualrechten, der Freiheit und Selbstbestimmung ermöglicht die Rechtsstellung des Bürgers wird auf diese Weise ebenfalls klar definiert. 
Die Freiheit des Einzelnen wird im Grundgesetz gleich zu Anfang behandelt. Das Grundgesetz stattet dabei seine Bürger in den ersten 17 Artikeln mit einem umfangreichen Arsenal an Freiheitsrechten aus, die jeden Einzelnen vor Zugriffen durch andere und auch durch den Staat schützen sollen. Ausgangspunkt ist dabei die Menschenwürde, die in Art.1 (1) GG als unantastbar beschrieben wird, und die von »aller staatlichen Gewalt» (Art.1(2) GG) zu schützen ist. In Absatz 3 wird zudem darauf verwiesen, dass die nachfolgenden Grundrechte »als unmittelbar geltendes Recht« (Art. 1(3) GG) die drei Gewalten des Staates binden. Der gesamte zweite Artikel widmet sich der Freiheit der Person, die eng mit der Menschenwürde verzahnt ist. Freiheit bedeutet in diesem Kontext in der Regel Selbstbestimmung (vgl. u.a. Löw 1997): »(1) Jeder hat das Recht auf die freie Entfaltung seiner Persönlichkeit, soweit er nicht die Rechte anderer verletzt und nicht gegen die verfassungsmäßige Ordnung oder das Sittengesetz verstößt. (2) Jeder hat das Recht auf Leben und körperliche Unversehrtheit. Die Freiheit der Person ist unverletzlich. In diese darf nur auf Grund eines Gesetzes eingegriffen werden« (Art. $2 \mathrm{GG}$ ).

Die darauf folgenden Grundgesetzartikel umfassen einen ganzen Katalog an klassischen liberalen Freiheits- und Abwehrrechten (siehe Abbildung 9). Sie sind ein wichtiger Bestandteil von Grundrechtskatalogen für moderne Verfassungsstaaten. Die Gleichheit vor dem Gesetz nach Art. 3 stellt sicher, dass die Bürger geschützt sind vor willkürlichen Maßnahmen des Staates im Bereich aller drei Gewalten. Männer und Frauen sind gleichberechtigt (vgl. Abs. 2) und niemand darf auf Grund bestimmter Merkmale benachteiligt oder bevorzugt werden. Artikel 4 des Grundgesetzes legt fest, dass es keine Staatsreligion in der Bundesrepublik gibt und dass jeder seine eigene Religion ohne Störungen ausüben kann. Ebenso wie die Religion wird des Weiteren auch die Meinung 


\begin{tabular}{|c|c|c|}
\hline Art. 3 GG & Gleichheit vor dem Gesetz & Art. $9 \mathrm{GG}$ Vereinigungsfreiheit \\
\hline \multirow[t]{2}{*}{ Art. 4 GG } & Glaubens- und Gewissensfreiheit, & Art. 10 GG Post- und Fernsprechgeheimnis \\
\hline & Kriegsdienstverweigerungsrecht & Art. 11 GG Freizügigkeit \\
\hline \multirow[t]{2}{*}{ Art. 5 GG } & $\begin{array}{l}\text { Recht der freien Meinungsäuße- } \\
\text { rung, Informationsfreiheit, Freiheit }\end{array}$ & Art. 12 GG Freiheit der Berufswahl \\
\hline & von Wissenschaft und Kunst & Art. 14 GG Eigentum \\
\hline Art. $6 \mathrm{GG}$ & Erziehungsrechte der Eltern & Art. 16 GG Auslieferungsverbot, Asylrecht \\
\hline Art. $8 \mathrm{GG}$ & Versammlungsfreiheit & Art. 17 GG Petitionsrecht \\
\hline
\end{tabular}

Abbildung 9 Klassische liberale Freiheits- und Abwehrrechte

eines jeden Bürgers geschützt - frei von Zensur darf die persönliche Meinung nach Art. 5 frei geäußert werden, allerdings mit kleinen Einschränkungen was ehrenrührige Behauptungen und den Schutz der Jugend angeht. Umgekehrt darf sich jeder auch aus freien Quellen unterrichten (vgl. u.a Hesselberger 1999: 93), was letztlich die Basis und auch die Bedingung für das Äußern von Meinungen ist. Nach Art. 6 stehen Ehe und Familie als wichtige private Kernbereiche unter dem Schutz des Staates, wobei die Eltern das Recht und die Pflicht für die Pflege und Erziehung ihrer Kinder haben. Dies gilt für eheliche ebenso wir für nichteheliche Kinder. Der achte Artikel des Grundgesetzes birgt einigen Zündstoff; er steht für Meinungsfreiheit, die in Bewegung gekommen ist. Es gilt seit jeher ein wichtiges Recht für unabhänige, politische mündige freie Bürger sich frei und ohne Waffen zu versammeln. Die Ereignisse der deutschen Vereinigung zeigen die politische Kraft, die hinter der Ausübung dieses Freiheitsrechts stehen kann. Der Artikel 9 geht noch einen Schritt über das Versammlungsrecht hinaus und garantiert die Koalitionsfeiheit, also das Recht, Vereine und Gesellschaften zu bilden. Regelmäßige Aufmerksamkeit 
findet dieses Recht bei Tarifverhandlungen, wenn Arbeitgeber und Arbeitnehmer über entsprechende Vereinigungen versuchen, die jeweiligen Interessen durchzusetzen. Der Staat oder Teile davon treten, wenn überhaupt, nur als betroffene Tarifpartei oder - nach Aufforderung - als Schlichter auf.

Als Maßnahme gegen Bespitzelung, wie sie beispielsweise zur Zeit des Nationalsozialismus üblich war (vgl. ebd.: 133) wurde mit Artikel 10 das Brief-, Post- und Fernmeldegeheimnis etabliert - weder staatliche Stellen noch die Briefbeförderungsunternehmen dürfen die Diskretion verletzen. Der ergänzende, einschränkende Absatz wurde mit den Notstandgesetzen angefügt.

Im Gegensatz zu vielen anderen Grundrechten ist die Freizügigkeit des Artikels 10 kein Menschenrecht, sonden ein Bürgerrecht. Es steht somit allen Deutschen frei, ihren Aufenthaltsort im Bundessgebiet frei zu bestimmten, Asylbewerber beispielsweise unterliegen diesbezüglich Beschränkungen. Für die Bürger der Europäischen Union gilt dieses Recht für das ganze Unionsgebiet. Eng damit ist die Freiheit der Berufswahl, die der Artikel 12 garantiert, und die ihre Grenzen an persönlichen Qualifikationsmerkmalen und Eignungen findet.

Das, was sich die Bürger erarbeiten und darüber hinaus auch alles Eigentum insgesamt, wird durch Artikel 14 geschützt. Zwar verpflichtet Eigentum, trotzdem ist es beispielsweise vor Enteignung in der Regel geschützt und es kann auch an nachfolgende Generationen weitervererbt werden.

All diese Rechte stehen den Deutschen zur Verfügung und der 16. Artikel sichert die Bürger insofern ab, als die Staatsbürgerschaft nicht entzogen werden darf und man als Deutscher auch nicht an das Ausland ausgeliefert werden darf. Abgerundet wird dieser Abschnitt durch das Petionsrecht des Artikels 17, das es allen Deutschen gestattet, sich in Form von Petitionen an 
entsprechende Stellen zu wenden. Dieses bis auf die Bill of Rights zurückzuverfolgende Recht (vgl. HESSELBERGER 1999: 164) ist nicht so unbedeutend, wie es auf den ersten Blick erscheint. Die angesprochenen Stellen sind verpflichtet, angemessen zu antworten: Der Bundestag unterhält aus diesem Grunde einen eigenen Petitionsausschuss, in dem politische Interessen von der politischen Basis ausgehend artikuliert werden. Bestimmte Ideen werden auf diesem Wege erstmalig einer breiteren politischen Öffentlichkeit präsentiert.

In den Artikeln 17a-19 werden gewisse Einschränkungen der Grundrechte ermöglicht, wenn diese »zum Kampf gegen die freiheitliche demokratische Grundordnung" (Art. $18 \mathrm{GG}$ ) verwendet werden. In jedem Falle müssen die Einschränkungen auf einem Gesetz basieren, das für alle gilt, darüber hinaus muss der Rechtsweg allen Bürgern offen stehen, die sich durch öffentliche Gewalt in ihren Rechten verletzt sehen (vgl. Art.19GG). Wie wichtig die verschiedenen Freiheitsrechte in der Praxis sind, wird deutlich, wenn man sich ihr Fehlen vorstellt. Nur allzu leicht ist ein ein politisches System vorstellbar, indem dem Einzelnen vorgegeben werden kann, was er arbeitet, wie er seine Kinder erzieht, wo er sich aufzuhalten hat und vieles mehr. Jede Meinungsäußerung unterläge der Zensur und der Einflussnahme. Die besondere Crux dabei wäre die Tatsache, dass das betreffende politische System sogar nicht automatisch eine Diktatur oder Ähnliches sein müsste. In jedem Fall müssten seine Bürger in einem System der Unsicherheit leben, da immer die Möglichkeit von willkürlicher Einflussnahme gegeben wäre. Eingriffe in das persönliche Leben wären grundsätzlich möglich und auch legal: Insofern garantieren die Freiheits- und Abwehrrechte nicht nur die jeweiligen Rechte, sondern zusätzlich Berechenbarkeit und Verläßlichkeit des politischen Systems. 
Insgesamt erweist sich deshalb das Grundgesetz als eine Verfassung, die sich klar an der Maxime der Freiheit orientiert. Für Menschen, die sich nicht professionell mit der Materie beschäftigen, geraten die Grundrechte und ihre Bedeutung für den Einzelnen leicht in Vergessenheit und verlieren an alltäglicher Relevanz und Präsenz, obwohl sie, wie die Geschichte zeigt, auf keinen Fall selbstverständlich sind, sondern den »Freiheitsraum des einzelnen vor Übergriffen der öffentlichen Gewalt« (РÖTzSCH 2001: 15) schützen.

Die Freiheitsrechte des Grundgesetzes stehen nicht isoliert, sondern sie sind in größere verfassungsrechtliche Strukturen eingebettet. Ein Sonderfall ist dabei der Artikel 20 des Grundgesetzes, der äußerst kompakt eine Vielzahl an wichtigen Regeln - fast schon wie in einer Miniaturverfassung - zusammenfasst: Benannt werden die Prinzipien Demokratie, Sozial- und Bundesstaatlichkeit, die Ausübung der Staatsgewalt durch das Volk in Form von Wahlen und Abstimmungen, die Gewaltenteilung sowie die Bindung der drei Gewalten an Recht und Gesetz. Zusammen mit den Grundsätzen des Artikels 1 und der Mitwirkung der Länder an der Gesetzgebung wird der Artikel 20 durch die Bestimmungen des Artikel 79 von Grundgesetzänderungen ausdrücklich ausgenommen, während alle anderen Artikel mit Zweidrittelmehrheit in Bundestag und Bundesrat prinzipiell abgeändert werden können. Betrachtet man das Grundgesetz in voller Gänze, so kann man es zurecht nach einer Urteilsbegründung des Bundesverfassungsgerichts als freiheitlich-demokratische Grundordnung einstufen:

"So läßt sich die freiheitliche demokratische Grundordnung als eine Ordnung bestimmen, die unter Ausschluß jeglicher Gewalt und Willkürherrschaft eine rechtsstaatliche Herrschaftsordnung auf der Grundlage der Selbstbestimmung des Volkes nach dem Willen der jeweiligen Mehrheit und der Freiheit und Gleichheit darstellt. Zu den Grundlegenden Prinzipien dieser Ordnung sind mindestens zu rechnen: 
- die Achtung vor den im Grundgesetz konkretisierten Menschenrechten, vor allem vor dem Recht der Persönlichkeit auf Leben und freie Entfaltung,

- die Volkssouveränität,

- die Gewaltenteilung,

- die Verantwortlichkeit der Regierung,

- die Gesetzmäßigkeit der Verwaltung,

- die Unabhängigkeit der Gerichte,

- das Mehrparteienprinzip und

- die Chancengleichheit für alle politischen Parteien mit dem Recht auf verfassungsmäßige Bildung und Ausübung einer Opposition.« (BVerfG 2, 12f., zitiert nach SutoR 1994: 110)

Das Bundesverfassungsgericht versteht die freiheitlich-demokratische Grundordnung nicht als statische, sondern geht davon aus, dass sie »verbesserungsfähig und -bedürftigt« (SUTOR 1994: 110) sei. Die Mitglieder dieser politischen Ordnung gestalten den dynamischen Wandel mit, wobei nicht a priori vorgegeben sein kann und darf, dass ein bestimmter Zustand das endgültige Ziel der Entwicklung sei (vgl. ebd). Die Freiheit der bei den Gemeinschaftsentscheidungen mitbestimmenden Mitglieder der Gesellschaft ist die Grundlage für diesen kontinuierlichen Wandel: Nach SUTOR ergeben sich aber aus diesen freien Entscheidungen auch Konfliktpotenziale, die letztlich ihre Ausformung im Kampf um politische Macht finden (ebd.: 111), wobei die staatliche Ordnung trotzdem so beschaffen ist, das Wohl möglichst vieler im Auge zu behalten. Das Eindämmen von Machtmissbräuchen, etwa in Form von Individualrechten und dem Schutz von Benachteiligten, ist dabei von größter Bedeutung. Das dazu gehörige Gegenstück ist die Akzeptanz von Mehrheitsentscheidungen durch die Unterlegenen im politischen Prozess, wobei allerdings diese Akzeptanz aber auch darauf beruht, dass jede Minderheit auch die Chance besitzt, einmal selbst zur Mehrheit werden zu können, um dann ihre Interessen realisieren zu können. 
Zusammenfassend mit Blick auf das gesamte Grundgesetz kann man sagen, dass Freiheit ein essentieller Bestandteil des Grundgesetzes ist und dass ihr sehr viel Raum in den Formulierungen eingeräumt wird. Es ist sicher kein Zufall, dass der Katalog an Grund- und Freiheitsrechten gleich am Anfang steht und mit der Menschenwürde beginnt, die allen weiteren Bestimmungen vorangestellt und übergeordnet ist. Die Erfahrungen der Weimarer Zeit und des Nationalsozialimus mögen mit ein Grund gewesen sein, warum diese Ausrichtung des Grundgesetzes erfolgte. Die Freiheitsrechte sind klassische Abwehrrechte gegen Eingriffe durch Dritte, was sowohl andere Bürger als auch staatliche Einrichtungen mit einschließt. Die politische Organisation ist so konzipiert, dass bei entsprechenden Freiheitseinschränkungen Rechtswege garantiert sind, die es ermöglichen, diese wieder aufzuheben.

\subsubsection{Sicherheit im Grundgesetz}

Im Gegensatz zur Freiheit findet sich im Grundgesetz kein Artikel, den man unmittelbar als ein explizites Grundrecht auf Sicherheit bezeichnen könnte, allenfalls für den Bereich der äußeren Sicherheit eröffnet Artikel 24 die Möglichkeit, dass die Bundesrepublik Hoheitsrechte auf zwischenstaatliche Einrichtungen übertragen und sich zudem in ein System »gegenseitiger kollektiver Sicherheit« (Art.24GG) einordnen kann, um eine »friedliche und dauerhafte Ordnung in Europa und zwischen den Völkern der Welt« (ebd.) zu sichern. Obgleich nirgends im Grundgesetz ein Grundrecht auf Sicherheit ausdrücklich erwähnt ist, wird zuweilen der Versuch unternommen, ein solches indirekt aus den Bestimmungen abzuleiten. In der Tat lassen sich einige Stellen innerhalb der Verfassung festmachen, an denen Sicherheit ableitbar 
und indirekt stets mitgedacht wird. Dies gilt schon für Artikel 1, der ausdrücklich auch den Staat erwähnt und die staatliche Gewalt verpflichtet, die Menschenwürde zu achten und zu schützen. Auch in Artikel 3 wird der Staat zum Partner und verpflichtet, aktiv an der Durchsetzung der Gleichberechtigung und an der Beseitigung bestehender Nachteile mitzuwirken. In analoger Form gilt dies auch für den Bereich Ehe und Familie (Art. 6 GG) und Schule (Art. 7 GG). Der Staat wird aber auch zum begrenzenden Element, so beispielsweise in Art. 10 (2), 11 (2), 12a (3-6), 17 und 18, in denen die Grenzen der jeweiligen Grundrechte geregelt sind. Der wichtige Grundgesetzartikel 20, der zentrale Staatsstrukturprinzipien verankert, erwähnt ebenfalls die Staatsgewalt, die vom Volk in Wahlen und Abstimmungen und durch die besonderen Organe der Legislative, Exekutive und Judikative ausgeübt wird. Die angesprochenen Ausführungen des Grundgesetzes sind Anknüpfungspunkte, die es nahelegen, nach einem immanenten, ableitbaren Grundrecht auf Sicherheit zu suchen. Der fundierteste, systematische und sehr einflussreiche Versuch einer solchen Ableitung eines Grundrechts auf Sicherheit wurde im Jahr 1983 von JOSEF ISENSEE unternommen, auf dessen Überlegungen im Folgenden Bezug genommen wird. ISENSEE geht dabei davon aus, dass die Grundrechte des klasssisch-liberalen Typus, den »status negativus« (ISENSEE 1983: 2) konstituieren. Dieser Begriff meint den Zustand, in dem die Bürger einer politischen Ordnung Abwehrrechte gegen den Staat erkämpft haben und letztlich in einem liberalen Rechtsstaat leben. Die Sicherheit, die der Staat vor allem durch den Einsatz seiner Behörden schafft, kollidiert mit diesen verbrieften Grundrechten. ISENSEE dreht aber nun die Blickrichtung um und sieht den Staat nicht nur als Bedrohung, sondern vielmehr als Garanten für die Aufrechterhaltung der rechtlichen Rahmenbedingungen von Freiheit (ebd.: 32). Für den 
Staat besteht eine Schutzpflicht (auch aufgegriffen von NEUSEL 1996: 90), aus deren Gesamtheit ISENSEE das Grundrecht auf Sicherheit ableitet (ebd.: 33). Daraus erwächst nach ISENSEE eine dauerhafte Verantwortung des Staates für die Sicherheit seiner Bürger, wobei sich die nötigen Maßnahmen analog zu den sich verändernden Gefahren wandeln müssen:

»Die Sicherheit, die der Staat anzustreben hat, ist ohnehin mehr als ein Bündel rechtlicher Maßnahmen, mehr auch als die Rechtsordnung insgesamt. Sie ist ein realer Friedens-Zustand des Gemeinwesens, der Grundvertrauen im Zusammenleben vermittelt und damit jedermann die seelische Unbefangenheit ermöglicht, sich frei zu bewegen.« (ISENSEE 1983: 40)

Totale Sicherheit, so ISENSEE weiter, sei nicht das Ziel der grundrechtlichen Schutzpflichten, da der Staat keinesfalls so präsent sein könne, um allumfassend alle Gefahren zu eliminieren. In jedem Fall seien die Basis für entsprechendes sicherheitsorientiertes Handeln gesetzliche Regelungen, die zudem den »Grundrechten der Betroffenen« (ebd.: 44) standhalten müssten und sich an den Prinzipien Erforderlichkeit, Geeignetheit und Verhältensmäßigkeit (ebd.: 46) zu orientieren hätten:

»Die Aufgabe des Rechtsstaates (...) läßt sich (...) dahin bestimmen: die rechtlichen Bedingungen herzustellen, unter denen die Willkür des einen mit der Willkür des anderen nach einem allgemeinen Gesetz der Freiheit zusammen vereinigt werden kann. Zur Grundrechts-Koordination gehört auch das Wächteramt des Rechtsstaats über die allseitige Respektierung der Freiheitsgrenzen. Die grundrechtlichen Schutzpflichten konvergieren also mit dem klasssisch liberalen Sicherheitszweck« (ebd.:46f.)

Nach ISENSEE ist es von Bedeutung, dass dem Staat nicht das Recht zukomme, dem Bürger seinen Schutz zu oktroyieren und so den Schutz zur Bewachung ausarten zu lassen. Sofern es legitim sei, also insofern keine Rechte Dritter betroffen sind, müsse dem Einzelnen letztlich die Möglichkeit verbleiben, den Schutz des Staates abzulehnen (ebd.: 49). 
Der letzte Gedanke ist ein zentraler Punkt, der heute in die immer noch andauernde und zum Teil scharf geführte Diskussion eingebracht wird. Autoren wie beispielsweise ALBRECHT (2003: 10-13) kritisieren das Grundrecht auf Sicherheit als »politische Kunstfigur» (ebd.: 12), die als »bewusster Gegensatz« zu den »Abwehrrechten gegen staatliche Macht« (ebd.: 13) etabliert werden soll. Die Blickrichtung wird erneut revidiert und ALBRECHT stellt die »radikalste Umkehrung des Verhältnisses von Staat und Bürger« (ebd.) fest, aus dem sich der staatliche Anspruch ableiten würde, dass die Bürger sich ordnungsgemäß zu verhalten hätten, was in letzter Konsequenz hin zu einer Diskussion um Bürgerpflichten führt. Das Grundrecht auf Sicherheit würde sich vollkommen ohne verfassungsrechtliche Grundlage zu einem »Supergrundrecht« (ebd.: 12) aufblähen, das auf den nun scheinbar einklagbaren Schutzpflichten des Staates beruhen würde. Der Versuch, das »humane Restrisiko« (ebd. 13) zu minimieren, führe dazu, dass Sicherheit zu Lasten der Freiheit kontinuierlich expandieren würde.

Das Grundgesetz räumt in der Gesamtschau der Freiheit einen wesentlich höheren Stellenwert ein als der Sicherheit. Während Freiheitsrechte klar formuliert sind, lässt sich kein explizites Grundrecht auf Sicherheit finden, sondern allenfalls ableiten. Sicherheit hat in diesem Kontext keinen verfassungsrechtliche Rang. Auch die von ISENSEE postulierte Schutzpflicht des Staates, der als Garant der Rahmenbedingungen für Freiheit (ISENSEE 1983: 46 f.) auftritt, ist nicht unmittelbar verfassungsrechtlich klar formuliert. Die politische Bedeutung von Sicherheit ist demnach nicht im Rahmen der Verfassung zu sehen. Sie ist vielmehr ein politisches Ziel und damit ein legitimer Teil der politischen Alltagspraxis, als der sie den normalen demokratischen Willensbildungsprozessen unterliegt. Dies ist für sich genommen 
keineswegs problematisch. Wie für vieles gilt das Motto: Weniger ist mehr - es muss auch keineswegs jedes politische Ziel Verfassungsrang bekommen und klar ausformuliert verankert werden. Problematisch (vgl. ALBRECHT 2003: 11) ist in diesem Zusammenhang nur, wenn man sich in der berechtigen politischen Auseinandersetzung mit dem Thema Sicherheit auf die Verfassung beruft, denn dort findet sich im Grunde keine unmittelbare Grundlage.

\subsubsection{Wichtige politische Akteure und Abläufe}

Zum Abschluss des Unterkapitels 3.1 soll nun ein kurzer Überblick über die wichtigsten politischen Akteure und die wirksamen politischen Abläufe gegeben werden. Dieser Überblick ist - obwohl er gewissermaßen sehr auf Grundlegendes ausgerichtet ist und sozialkundlich erscheinen mag - notwendig und sinnvoll, um ein zusammenhängendes Bild von der Ausgangslage hinsichtlich Sicherheit und Freiheit der Bundesrepublik Deutschland zu erhalten. Die sich anschließenden wichtigen Veränderungen, die sich über alle sechs Jahrzehnte der Bundesrepublik Deutschland vollzogen, werden vor diesem Hintergrund letztlich erst kontrastreich erkennbar.

Die in Artikel 20 festgelegte Bundesstaatlichkeit wird in der Bundesrepublik über das Zusammenspiel aus Bund und Ländern realisiert. Nach Artikel 83 führen dabei die Länder die Bundesgesetze als eigene Angelegenheit aus, soweit im Grundgesetz keine anderen Bestimmungen zu finden sind. Dieser Grundsatz gibt den Ländern ein Exekutivgegengewicht zum Primat des Bundes in der Gesetzgebung (vgl. DitTMANN 2003: 1695), der den Ländern nur geringe legislative Rechte etwa im Bereich des Kultus belässt. Diese Aufteilung der Macht wird als vertikale Gewaltenteilung (vgl. ReUTER 1990:127) bezeichnet, 
welche die klassische horizontale Gewaltenteilung in Legislative, Exekutive und Judikative funktional ergänzt. Nach REUTER wirkt diese »doppelte Gewaltenteilung" (ebd.) vorbeugend gegen staatlichen Machtmissbrauch und dadurch »freiheitssichernd« (ebd.). Das Grundgesetz legt in diesem Kontext eine ganze Reihe von Verfassungsorganen fest, die gemeinsam für die "Koordination politischer Gestaltung" (SCHMIDT-PREUSS 1999: 467) sorgen.

Eine Sonderstellung nimmt dabei der Bundestag ein, der als einziges Verfassungsorgan seine politische Legitimation und Macht direkt vom Volk (vgl. EschenBurg 1956: 496) ableiten kann. Die Grundlage dieser Legitimität ist auf der Bundesebene die Wahl nach dem personalisierten Verhältniswahlrecht - über die Erststimme wird ein Direktkandidat und über die bedeutsamere Zweitstimme (5\%-Hürde) eine Partei gewählt (vgl. KORTE 2003: 43). Innerhalb des politischen Gefüges nimmt der Bundestag als deutsches Parlament eine Fülle von Aufgaben wahr, die nach ISMAYR in vier Bereiche unterteilt werden können: Rekrutierungsfunktion, Gesetzgebungsfunktion, Kontroll- und Initiativfunktion, Repräsentations- und Kommunikationsfunktion. Die Art und Weise wie die politische Praxis des Bundestags gestaltet wird, ist durch eine so genannte »symptomatische funktionale Dualität« (SCHMIDTPreUSS 1999:472) geprägt. Dabei ist die Regierungsmehrheit des Parlaments der Exekutive gegenüber eher fördernd und unterstützend eingestellt, was erhebliche Auswirkungen beispielsweise auf die Wahrnehmung der Kontrollfunktion hat, die wesentlich kritischer und aggressiver von den Oppositionsfraktionen ausgeübt wird. Kontrolle und Kritik der Regierung wird durch die parlamentarische Regierungsmehrheit in der Regel eher informell ausgeübt und verlagert sich oftmals in Fraktionssitzungen und Parteigremien. Ganz anders funktionieren diese Mechanismen auf Seiten der Opposition, die das 
gesamte Arsenal parlamentarischer Kontrollrechte (z. B. Kleine und Große Anfrage, Aktuelle Stunde, Untersuchungsausschüsse, Enquête-Kommissionen etc.) benützen, nicht zuletzt, um die Chancen zu verbessern, selbst von der Oppositionsrolle in die Regierungsverantwortung wechseln zu können. Im Hinblick auf die Gewaltenteilung verläuft die entsprechende Teilungslinie mitten durch das Parlament.

Die wichtigste politische Gestaltungsaufgabe des Parlaments ist die Gesetzgebung, da das Gesetz die Grundlage allen rechtsstaatlichen Handelns ist. Im Prozess der Gesetzgebung wirkt allerdings auch der Bundesrat mit, der im Verlauf der geschichtlichen Entwicklung vermehrt über zustimmungspflichtige Gesetze zur wichtigen Entscheidungsinstanz wird. Das Besondere am Bundesrat ist nicht die Tatsache, dass die Bundesländer über eine zweite Kammer in den legislativen Prozess integriert sind - eine Alternative zum Bundesratsprinzip ist das Senatsprinzip, das beispielsweise in den USA existiert. Interessant ist vielmehr, dass ausgerechnet die Exekutiven der Bundesländer den Bundesrat bilden und damit legislativ tätig werden.

Nach Artikel 77 des Grundgesetzes greift der Vermittlungsausschuss, der aus Mitgliedern des Bundestags und des Bundesrats gebildet wird, ein, wenn der Bundesrat ein Gesetzesvorhaben gestoppt hat. Die Gründe für solch ein politisches Verhalten kann sachliche oder taktische Gründe haben, auch Koalitionsvereinbarungen auf der Ebene der Länder können eine Rolle spielen. Aus Transparenzgründen ist es dabei problematisch, dass den sich anschließenden Entscheidungen des Vermittlungsausschusses keine inhaltlichen Begründungen beigefügt werden, die Auskunft über die internen Abläufe geben würden. Sowohl Bundestag als auch Bundesrat besitzen das Initiativrecht, mit dem sie den Gesetzgebungsprozess starten können. Auch die Bundesregierung besitzt 
dieses Recht und übt es in enger Zusammenarbeit mit den Bundestagsfraktionen, die dem Regierungslager angehören, aus.

Da die primäre Funktion der Regierung die Staatsleitung ist (SCHAMBECK 1999: 451) und rechtliche Regelungen die Basis für staatliches Handeln sind, ist diese Kooperation von Regierung und Regierungsmehrheit von einiger praktischer Bedeutung. Gleichzeitig haben die Ministerien, die einen Großteil der Regierungsvorlagen ausarbeiten, aber einen enormen personellen, sachlichen und finanziellen Vorsprung vor den Abgeordneten, die mit ihren wenigen Ressourcen kaum mithalten können. Im Mittelpunkt der Exekutive steht der Kanzler, der durch seine Richtlinienkompetenz (Art.65GG) die Richtung des operativen politischen Tagesgeschäft bestimmt. Diese Kompetenz ist aber kein Selbstläufer, sondern die Persönlichkeit des Kanzlers, die Mehrheitsverhältnisse im Parlament und die politischen Partner sind ausschlaggebende Einflussfaktoren auf die Umsetzung. Innerhalb des Bundeskabinetts gilt zudem das Ressortprinzip, das besagt, dass jeder Minister seinen Bereich eigenverantwortlich leitet. Der Bundespräsident ist im Vergleich zu seinen präsidialen Vorgängern in seiner Machtfülle reduziert worden. Er wird von der Bundesversammlung (Art.54GG) gewählt und ist der oberste Repräsentant der Bundesrepublik. Er kann Begnadigungen vornehmen, ernennt auf Vorschlag den Kanzler und Minister und soll über die Parteigrenzen hinweg integrierend wirken. Mit seiner Unterschrift und der anschließenden Veröffentlichung im Bundesgesetzblatt endet der legislative Prozess, allerdings besitzt er lediglich ein formales Prüfungsrecht, das er vor der Unterzeichnung ausübt.

Die dritte Gewalt ist die Judikative, an deren Spitze neben den obersten Bundesgerichten (Art. 95 und 96 GG) das Bundesverfassungsgericht steht. Für 
diese Untersuchung ist in erster Linie das Letztere relevant, weil einerseits das Bundesverfassungsgericht in seinen Urteilsbegründungen ausführlich dazu Stellung genommen hat, was die freiheitliche demokratische Grundordnung in Deutschland ausmacht (vgl. LaUtner 1982: $3 \mathrm{ff}$.). Zudem kann die Bedeutung des Bundesverfassungsgerichts kaum überschätzt werden, es wird zuweilen gar als »Krönung des Rechtsstaates« (z.B. CLEMENS 1995: 14) bezeichnet. Andere Autoren wie Ueberschaer (1962) widmen sich in gleichem Sinne der Suprematie des Gerichts. Es stellt sich nun die Frage, wie es dazu kommt, dem obersten deutschen Gericht eine solche Bedeutung beizumessen. In jedem Fall spielen die Aufgaben, die das Gericht wahrnimmt, eine besondere Rolle. Nach SÄCKER (1998:55-73) kann man diese in vier Bereiche unterteilen: So kümmert sich das Bundesverfassungsgericht um Verfassungsbeschwerden, mit denen der einzelne Bürger seine Grundrechte »wahren und durchsetzen« (ebd.: 55) kann. Jedermann, der sich durch »die öffentliche Gewalt in seinen Grundrechten verletzt« (ebd.) fühlt, kann das Gericht anrufen, wobei neben natürlichen Personen in leicht eingeschränktem Umfang auch juristische Personen und Gemeinden dazu berechtigt sind (ebd.: 56 f.). Konkret können sich Verfassungsbeschwerden gegen Gesetze, Behördenakte bei völliger Ausschöpfung des Rechtsweges und Urteile von anderen Gerichten richten. Im Bereich der Normenkontrolle kann nur das Bundesverfassungsgericht überprüfen, ob bestimmte gesetzliche Regelungen höherrangigem Recht widersprechen, wobei unterschieden wird zwischen konkreter Normenkontrolle, die durch einen Gerichtsbeschluss bei einem konkreten Rechtsfall eingeleitet (vgl. ebd.: 63) wird, und der abstrakten Normenkontrolle, die auf Antrag von Bundesregierung, einer Landesregierung oder von mindestens einem Drittel des Bundestages (ebd.: 65) gestellt werden kann und sich mit 
der Vereinbarkeit von Normen auf unterschiedlicher Ebene beschäftigt. Verfassungsstreitigkeiten zwischen staatlichen Organen (Art. 93 GG) - darunter fallen Organstreitigkeiten, Bund-Länder-Streitigkeiten und Landesverfassungsstreitigkeiten - sind der dritte Bereich, in dem das Gericht entscheidet. Unter den weiteren Aufgaben des Bundesverfassungsgerichts fasst SÄCKER (ebd.: 71 ff.) Parteiverbotsverfahren, Grundrechtsverwirkungen, Wahlprüfungsverfahren, Anklage gegen den Bundespräsidenten und Richter und Gutachtenverfahren zusammen.

Das Bundesverfassungsgericht hat nicht nur unmittelbar durch die oben stehenden Zuständigkeiten eine so enorme bedeutsame Stellung im politischen System der Bundesrepublik. Die Mitglieder des Gerichts, das über zwei Senate organisiert ist, haben langfristige Amtszeiten und üben ihre Tätigkeit mit der nötigen richterlichen Unabhängigkeit aus. Obwohl in den ersten Jahrzehnten der Republik durch die Parteien versucht wurde, das Gericht über die Wahl der Richter durch Bundestag und Bundesrat parteipolitisch zu beeinflussen (WESEL 1996: $16 \mathrm{ff}$.), nahmen die auf die Weise berufenen Richter sehr schnell ihre neue überparteiliche Rolle an und lockerten die "Loyalitäten « zu den politischen Parteien (vgl. Rollecke 1999: 34). Das oberste deutsche Gericht wird in vielerlei Hinsicht entscheidend auf die Entwicklung von Sicherheit und Freiheit einwirken, dementsprechend wird im weiteren Verlauf der Arbeit noch weiter darauf eingegangen werden müssen.

Die drei Gewalten sind in der Bundesrepublik Deutschland nicht im klassischen LOCKE'SCHEN oder MONTESQUIEU'SCHEN Sinn geteilt. Sie werden durch das Grundgesetz als gleichwertig angeordnet, sind aber nicht isoliert, sondern müssen sich im politischen Alltag gegenseitig kontrollieren können. Diese Aufgabe wird im politischen Altag in einer ganzen Reihe von »Überschneidungen 
und Verflechtungen« (Löw 1997: 220) konkretisiert, die man auch als Gewaltenverschränkung bezeichnen kann. Dazu zählt etwa der Umstand, dass die Regierungsmitglieder in der Regel auch dem Bundestag angehören, der Bundestag zuweilen Gesetze erlässt, die de facto nur einen Einzelfall entscheiden, Rechtsverordnungen durch die Regierung, die große Anzahl an Gesetzesvorlagen durch die Regierung und die Wahl der Verfassungsichter durch Bundestag - hier durch einen nach dem Verhältnisprinzips zusammengesetzten Zwölferausschuss - und dem Bundesrat (vgl. ebd.). Ebenfalls wirksame Verschränkungen sind der konstruktive Misstrauensantrag und die Vertrauensfrage, mit denen Exekutive und Legislative eng verzahnt sind.

Betrachtet man die Alltagspraxis, so wird schnell deutlich, dass das gesamte politische System von den Parteien überlagert ist, die eigentlich nach Artikel 21 des Grundgesetzes bei politischen Willensbildung lediglich mitwirken und haben erst durch die Legitimation von Wahlen an der staatlichen Gewalt Anteil (IPSEN 2003: 895). De facto dominieren sie die Rekrutierung des politischen Personals über Wahllisten, die Arbeit im Bundestag, wo viele Funktionen über die Fraktionen organisiert sind wie z.B. die Besetzung der für die politische Arbeit immens wichtigen Ausschüsse, vgl. ScHAMBECK (1999: 461), die Spitze der Exekutive, die im Wahlkampf und im politischen Alltag massiv von den Fraktionen und Spitzengremien (Koalitionsverträge) abhängig sind und nicht zuletzt die Wahl der obersten Richter ${ }^{1}$. Diverse wissenschaftliche Autoren greifen in diesem Zusammenhang den von LEıBHOLz geprägten Begriff des Partei-

1 Die besondere Problematik der Richterwahl und der personellen und finanziellen Ausstattung der gesamten Judikative wird später noch bewertet, wenn untersucht wird, wie sich die politische Struktur im Laufe der Jahrzehnte verändert hat. 
enstaates auf und diskutieren ihn kritisch, wenn bestimmte Phänomene und Probleme (vgl. u.a. SCHMIDT 2000: 379f.) der Parteien besprochen werden. Das Parteiensystem Deutschlands hat wie in vielen anderen westlichen Demokratien zunehmend mit Parteienverdrossenheit zu kämpfen, deren tatsächliche Ursachen (vgl. BREUER 1999: 322) vermutlich in der Entideologisierung der Parteien liegen und dem damit verbundenen Verlust der Bindung der Partei an ein bestimmtes politisches Klientel.

Neben den Parteien besteht im pluralistischen System Deutschlands Platz für diverse Gruppen, die wie die Parteien versuchen, ihre jeweiligen Interessen zu vertreten. Der dem Neopluralismus zuzurechende Politikwissenschaftler ERNST FRAENKEL spricht 1962 davon, dass es neben weiten Bereichen der Gesellschaft, in denen weitgehend Konsens besteht, es auch »Gebiete(.) des staatlichen und gesellschaftlichen Lebens gibt, über deren Regelung Meinungsverschiedenheiten zwischen verschiedenen Gruppen existieren (...)« (FRAENKEL 1972: 161). Dieser Umstand sei wünschenswert und wichtiger Bestandteil öffentlichen Lebens. Für die Freiheit ist dies wichtig, da aus den Konflikten (vgl. BENDA 1985: 5) und den damit verbundenen Lösungsstrategien sich Gestaltungsfreiräume für die Politik und der Bedarf an Toleranz entwickeln ${ }^{1}$. Die grundgesetzliche Basis für diese divergierenden, aber auch

1 Der Pluralismus ist innerhalb der politischen Theorie ein wichtiger und kontrovers diskutierter Ansatz. Eine übersichtliche Darstellung des Phänomens findet sich bei Nuscheler/Steffani (1972) und Steffan I (1977), darunter auch radikale Positionen wie die von HAROLD LASKI (1915). Grundlage für diese Arbeit ist der neopluralistische Ansatz ERnst Fraenkels, der Pluralismus notwendig mit einer freiheitlichen Verfassung verbindet, welche die pluralistische Vielfalt von Interessengruppen und Individuen erst ermöglicht (vgl. SONTHEIMER 1957: 256). 
organisierten Interessen sind die Artikel 8 (Versammlungsfreiheit) und 9 (Vereinigungsfreiheit). Besonders hervorgehoben wird im Art. 9 (3) das Recht zur »Wahrung und Förderung der Arbeits- und Wirtschaftsbedingungen«, was die Grundlage für Gewerkschaften und Arbeitgeberverbände ist, für welche die so genannte Tarifautonomie garantiert wird. Neben diesen klassischen Interessenverbänden treten weitere an, die sich allerdings in puncto Organisationsgrad (Bauernverband), Öffentlichkeitswirksamkeit (Greenpeace), finanzieller Ausstattung (IG Metall) und Prestige (ADRA) unterscheiden. Die Interessengruppen versuchen, ihren Anliegen Geltung zu verschaffen, indem sie öffentlichkeitswirksam arbeiten und versuchen, über die Medien Aufmerksamkeit zu erzeugen. Gleichzeitig wird punktuell versucht, unmittelbar auf die politischen Sachentscheidungsprozesse Einfluss zu nehmen, etwa indem einzelne Mandatsträger und Entscheidungsgremien strategisch mit bestimmten Informationen versorgt werden, um entsprechende Entscheidungen herbeizuführen. Derzeit sind am Deutschen Bundestag über 2000 Interessenverbände registriert, die in unterschiedlicher Intensität ihre Lobbyismustätigkeit ausüben

Parteien und anderen Interessengruppen kommt innerhalb des politischen Systems die Aufgabe zu, verschiedene Interessen aufzugreifen und zu bündeln, um so die Durchsetzungsfähigkeit der gesammelten Interessen zu erhöhen. Das politische System versucht, für alle beteiligten Interessengruppen die Rahmenbedingungen gleich zu gestalten. Nach Artikel 9 (2) GG sind dabei die verfassungsmäßige Ordnung und der Gedanke der Völkerverständigung wichtige Prinzipien. Rudzıo spricht in diesem Kontext zu Recht von einer »pluralistischen Demokratie« (RuDzı 2000: 69), welche die folgenden Elemente in sich vereinigt: 
- Es existiert eine Vielfalt organisierter Gruppen, die auf die staatliche Willensbildung Einfluss nehmen können;

- Interessen können frei artikuliert werden; in der politischen Praxis werden diese durch Mandatsträger repräsentiert;

- Interessen werden gebündelt, um die Chancen auf die Durchsetzung zu erhöhen;

- Die Beteiligung an Entscheidungen und die Akzeptanz von Entscheidungen sind wichtige Bestandteile.

Zusammenfassend kann man sagen, dass das Grundgesetz einen umfassenden Rahmen darstellt, auf dem der politische Alltag aufbaut. Die Staatsgewalt ist aufgeteilt in die klassischen drei Bereiche und es herrscht das Prinzip der gegenseitigen Kontrolle und Balance. Die Freiheit des Einzelnen ist in der Grundfassung des Grundgesetzes mit einem umfassenden Katalog von verschiedenen Rechten abgesichert, die zudem vor unabhängigen Richtern einklagbar sind. Die Väter des Grundgesetzes hatten, vermutlich aufgrund der Erfahrungen mit den vorherigen Verfassungen und der Zeit des Nationalsozialismus, diese weitgehenden Sicherungen eingebaut. Gleichzeitig wurde der konser-vative Charakter der Verfassung betont, in dem mit der Ewigkeitsklausel des Artikels 79GG der Verfassungskern völlig und die übrigen Bestimmungen mit relativ hohen Hürden - Zweidrittelmehrheit in Bundestag und Bundesrat - vor Änderungen geschützt wurden. Trotz dieses Schutzes vor Veränderungen wurde das Grundgesetz im Laufe der Jahrzehnte in einigen Artikeln verändert. Die folgenden Ausführungen werden sich mit den wichtigsten Änderungen befassen, soweit sie Auswirkungen auf die Sicherheit und die Freiheit hatten. 


\subsection{Die Notstandsgesetze}

Das, was alltäglich als Notstandsgesetze bezeichnet wird, meint präziser gesagt das 17. Gesetz zur Ergänzung des Grundgesetzes vom 24. Juni 1968, durch das insgesamt 17 Grundgesetzartikel verändert wurden. Diese Grundgesetzänderungen wurden zu ihrer Zeit heftig und kontrovers im Parlament und in der Öffentlichkeit - hier vor allem im Bereich der so genannten außerparlamentarischen Opposition (APO), die sich als Alternative zu den als schwach empfundenen Oppositionsparteien positionierte - diskutiert, sie konnten aber letztlich nicht durch deren gesellschaftliches Engagement und ihre Proteste verhindert werden. Die Eingriffe in das Grundgesetz waren tiefgehend, trotzdem sind die Auswirkungen auf Sicherheit und Freiheit aus der heutigen Perspektive ambivalent zu sehen, da ein großer Teil der Bestimmungen bislang noch nicht angewendet wurden.

\subsubsection{Die Ausgangslage}

Um die Bedeutung der Notstandsgesetze und ihre Auswirkungen auf Sicherheit und Freiheit korrekt einschätzen zu können, lohnt sich ein kurzer Blick auf die politische Entwicklung, welche die noch junge Bundesrepublik Deutschland zu dieser Zeit durchgemacht hatte, vor allem was die Erweiterung ihrer »eingeschränkten Souveränität« (WEBER 1981: 206) angeht. Ausgehend von der bedingungslosen Kapitulation Deutschlands bei Kriegsende und den danach installierten weitreichenden allierten Vorbehaltsrechten wurde Souveränität stückweise zurückerlangt. Dieser Prozess begann mit der Tätigkeit der Alliierten Hohen Kommission und der Verabschiedung des Besatzungsstatuts im Jahre 1949 und endete letztlich erst mit dem Friedensvertrag nach 
Abschluss der 2+4-Verhandlungen und der deutschen Vereinigung am 3. Oktober 1990. GREWE nennt die frühe Phase dieses Prozesses den »Übergang vom Besatzungsregime« (GREWE 1985: 9) und beschreibt ihn als »langwierig und kompliziert(en)«(GREWE 1988: 101) Prozess. Verschiedene Vorbehaltsrechte der Alliierten wurden zunächst installiert und dann über Jahrzehnte hinweg durch verschiedene Verträge abgebaut. Die Notstandsgesetze von 1968 sind dabei ein wichtiger Schritt, da durch sie die Bestimmungen des 2. und 5. Artikels des Deutschlandsvertrages von 1954 erfüllt werden konnten und entsprechende allierte Vorbehaltsrechte abgelöst wurden (vgl. Erklärung der Drei Mächte vom 17. Mai 1968) und nun in deutscher Hand lagen. Ein wichtiger Schritt hin zur Souveränität war damit vollzogen.

Neben den Grundgesetzänderungen von 1968 muss man ergänzend eine ganze Reihe von einfachen Notstandsgesetzen berücksichtigen, die noch vor 1968 von den verschiedenen Mehrheiten im Bundestag verabschiedet wurden (vgl. ausführlich dazu SCHÄFER 1966: 26 ff.). In der Regel wurden diese einfachen Gesetze mehr oder weniger unbemerkt von der breiten Öffentlichkeit geltendes Recht, obwohl auf fachlicher Ebene die Thematik durchaus diskutiert wurde. WALDMANN stellt die verschiedenen einfachen Notstandsgesetze übersichtlich vor und ordnet sie nach drei Hauptgruppen (vgl. WALDMANN $1968 \mathrm{ff}$, siehe Abbildung 10.). Bereits diese einfachen Notstandsgesetze wirkten freiheitseinschränkend, indem sie der Exekutive erlaubten, über Rechtsverordnungen bestimmte Verhaltensweisen der Bürger (Produktion von bestimmten Gütern, Dienstleistungen) zu erzwingen.

Die Notstandsgesetze des Jahres 1968 schlossen sich an die oben genannten einfachen Gesetze an. Sie wurden durch die Mehrheitsverhältnisse der großen Koalition unter KURT GEORG KIESINGER ermöglicht, welche die nötige 
Gesetze, die zum Schutze der Bürger gelten

- Erstes Gesetz über Maßnahmen zum Schutz der Zivilbevölkerung, Luftschutzwarndienst, freiwillige Mitarbeit bei verschiedenen Hilfsdiensten

- Gesetz über den Selbstschutz der Zivilbevölkerung, Gerätebeschaffung, Verdunkelung, Löschmittel, Haushaltsvorräte

- Gesetz über bauliche Maßnahmen zum Schutz der Zivilbevölkerung

- Gesetz über das Zivilschutzkorps, 200000 Mann Luftschutzhilfsdienst

Vorsorge zur Sicherstellung der Verteidigungsbereitschaft

- Wehrpflichtgesetz, Bundesreservisten für Bereitschaftsdienste (ohne Altersbeschränkung)

- Bundesleistungsgesetz, Leistungen und Anforderungen sachlicher Gegenstände im Verteidigunsfall

- Gesetz zur Ergänzung des Gesetzes über den Bundesgrenzschutz und die Einrichtung von Bundesgrenzschutzbehörden, Kombattantenstatus für Angehörige des Grenzschutzes bei Grenzverletzungen

Sicherstellungsgesetze (Vorsorgemaßnahmen)

- Wirtschaftssicherstellungsgesetz, Eingriffsermächtigung auf die Marktwirtschaft

- Ernährungssicherstellungsgesetz, Kontrolle von Produktion und Verteilung landwirtschaftlicher Produkte

- Wassersicherstellungsgesetz

- Verkehrssicherstellungsgesetz

Abbildung 10 Einfache Notstandsgesetze (abgeändert, vgl. WALDMANN 1968: $58 \mathrm{ff}$.)

Zweidrittelmehrheit in Bundestag und Bundesrat besaß. Die gesamte Entwicklung der verschiedenen Gesetze war nach OBEREUTER (1978:182) bereits in Artikel 111 des Herrenchiemseer Entwurfs beschrieben, daher ist es wohl falsch, davon auszugehen, dass bei der Urfassung des Grundgesetzes bewusst 
auf Notstandsgesetze verzichtet wurde (ebd.: 181) - von verschiedenen Gegnern der Notstandsgesetze wurde das Fehlen einer Notstandsgesetzgebung als bewusste Entscheidung der Väter des Grundgesetzes gegen solche Verfassungsinhalte gesehen. OBERREUtER geht stattdessen davon aus, dass dies auf Weisung der Alliierten geschehen war (ebd.: 183).

Der legislative Prozess, den die Notstandsgesetze durchliefen, wurde von einer aufgeheizten politischen Atmosphäre (vgl. GöRTEMAKER 2002: 207) begleitetet, was unter anderem durch das Dutschke-Attentat und die Osterunruhen (ebd.) bedingt war. Der große Konsens der Parteien bei der Entwicklung der Notstandsgesetzgebung - eingebracht wurden verschiedene Entwürfe, die immer wieder überarbeitet und im Hinblick auf die überparteiliche Kompromissfindung verändert wurden (vgl. WALDMANN 1968: 113) schaffte Raum für außerparlamentarische Formen der Opposition.

\subsubsection{Die einzelnen Veränderungen durch die Notstandsgesetze}

Das 17. Gesetz zur Ergänzung des Grundgesetzes vom 24.Juni 1968 umfasst 17 Grundgesetzänderungen, die in ihrer Gesamtheit heftig diskutiert wurden. Neben den Normalzustand, in dem das politische System wie gewohnt funktioniert, tritt nun der Notstand, der allerdings nicht als solcher direkt bezeichnet wird. Stattdessen kennt das Grundgesetz nach Art. 80a GG nun den Spannungsfall, der eine Vorstufe des Verteidigungsfalls darstellt und vom Bundestag mit Zweidrittelmehrheit festgestellt wird. Den Verteidigungsfall selbst stellt ebenfalls der Bundestag mit Zustimmung des Bundesrates auf Antrag der Bundesregierung fest, wenn das Bundesgebiet mit Waffengewalt angegriffen wird oder solch ein Angriff direkt bevorsteht (vgl. Art. 115 a GG). 
Die Befehlsgewalt über die Streitkräfte geht mit der Verkündigung des Verteidigungsfalls auf den Bundeskanzler über (Art. 115 b GG).

Generell gilt für den Weg der Gesetzgebung nach Art. 115 d (2) GG, dass von der Bundesregierung als dringlich bezeichnete Gesetzesvorlagen gleichzeitig dem Bundestag und Bundesrat zuzuleiten sind, die dann die Vorlagen gemeinsam beraten und mit Mehrheit entscheiden. Der Gemeinsame Ausschuss (vgl. Art. 53 a GG), der aus zwei Dritteln der Abgeordneten des Bundestages und Mitgliedern des Bundesrates besteht, wird im Verteidigungsfall tätig, wenn dem Zusammentreten des Bundestages unüberwindliche Hindernisse entgegen stehen. Nach Artikel 115 e GG kann der Gemeinsame Ausschuss selbst festsetzen, dass die Bedingungen dafür gegeben sind, dass er die Rechte von Bundestag und Bundesrat wahrnehmen kann.

Für den einzelnen Bürger änderten sich ebenfalls wichtige Bestimmungen. Das Brief-, Post und Fernmeldegeheimnis des zehnten Artikels wurde um Beschränkungen erweitert, die zwar durch ein Gesetz geregelt sein müssen aber - insofern die Beschränkungen »dem Schutz der freiheitlichen demokratischen Grundordnung oder des Bestandes oder der Sicherung des Bundes oder eines Landes« (Art. 10 (2) GG) dienen - den betroffenen Bürgern die Beschränkungen unter der Voraussetzung nicht mitgeteilt werden müssen, dass von der Volksvertretung bestimmte Organe die Nachprüfung übernehmen. Damit ist der klassische Rechtsweg für den Bürger in diesem Falle nicht möglich. Ebenfalls durch ein Gesetz kann die im Artikel 3 niedergelegte Freizügigkeit der Bürger nach den Grundgesetzänderungen unter bestimmten Voraussetzungen eingeschränkt werden (Art. 11 (3) GG). Nach Art. 115 C GG können zudem Entschädigungen für Enteignungen vorläufig geregelt werden, womit sie dann dem in Artikel 14 garantierten Rechtsweg 
entzogen werden. Gleichzeitig schafft die Notstandsgesetzgebung die Möglichkeit, Freiheitsentziehungen auf bis zu vier Tage auszudehnen (Art. 115 (2) GG), insofern kein Richter innerhalb der für Normalzeiten geltenden Frist tätig werden konnte. Die weiteren Bestimmungen der Notstandsgesetze widmen sich den Wahlperioden, dem Einsatz der Streitkräfte und der Bundespolizei im Inneren, der Hilfe für die Landesregierungen und letztlich der Beendigung des Verteidigungsfalles und dem Abschluss eines Friedensschlusses am Ende des Verteidigungsfalles. Während der gesamten Vorgänge bleiben die «verfassungsmäßige Stellung und die Erfüllung der verfassungsmäßigen Aufgaben des Bundesverfassungsgerichtes" (Art. $115 \mathrm{~g} \mathrm{GG)} \mathrm{unangetastet} \mathrm{und} \mathrm{dürfen} \mathrm{nicht} \mathrm{beeinträchtigt} \mathrm{werden.} \mathrm{Etwaige}$ gesetzliche Änderungen durch den Gemeinsamen Ausschuss, die das Bundesverfassungsgericht betreffen, können nur mit Zustimmung des Gerichts erfolgen (ebd.).

\subsubsection{Die Folgen für Sicherheit und Freiheit}

Um die Folgen der Notstandsgesetze auf Sicherheit und Freiheit einschätzen zu können, muss man einerseits verschiedene Argumente verknüpfen, die schon in der zeitgenössischen Diskussion aufs Tapet gebracht wurden. Andererseits ist es in der Rückschau ebenfalls wichtig, die konkrete Anwendung der Notstandsgesetze und eine mögliche latente Wirkung zu bewerten.

WALDMANN fasst 1968 die Anti-Notstandsgesetzgebungsargumente in fünf Hauptkategorien zusammen (vgl. WALDMANN 1968: 171 ff.):

- Keine Notwendigkeit, da die Sowjetunion und die Absichten der kommunistischen Bewegung friedliebend sind; 
- Künftige Kriege werden atomar geführt, es bleibt daher nichts übrig, was noch in Notstand geraten könnte;

- Innenpolitische Gefahren der Notstandsgesetzgebung, auch bezogen auf die vorangegangenen einfachen Notstandsgesetze;

- Keine Notwendigkeit, da vorhandene Regelungen ausreichen;

- Notstandsgesetzgebung wirkt gegenüber anderen Nationen als nach außen aggressiv wirkender Akt.

WALDMANN steht den von ihm vorgestellten Punkten kritisch gegenüber und ordnet sie korrekter Weise überwiegend KARL JASPERS zu, der sich 1966 äußerst kritisch zu der geplanten Notstandgesetzgebung äußert (vgl. JASPERS 1966: 157 ff.). Der hinter der vielschichtigen Argumentation JASPERS stehende wichtigste Gedanke ist die Befürchtung, dass der Ausnahmezustand durch legale politische Prozesse zum Normalzustand (vgl.ebd.: 173 f.) und die Herrschenden durch die nun ungebändigte Macht korrumpiert würden. Gut zehn Jahre später ist die Diskussion um die Notstandsgesetze weitgehend versachlicht. OBERREUTER bezeichnet 1978 das »rechtsstaatliche und demokratische Verfassungssystem, charakterisiert durch seine Freiheitsgarantien und sein offenes Verfahren politischer Willensbildung« (OBERREUTER 1978: 202) zum Schutzobjekt der Notstandsgesetzgebung, wobei er aber das fortdauernde Spannungsverhältnis von Freiheit und Macht durchaus wahrnimmt. Die Dualität von schnellen Entscheidungsstrukturen zusammen mit der Stärkung der Durchsetzungskraft der Staatsgorgane einerseits und der »Bewahrung rechtsstaatlicher Schranken« (ebd.: 203) andererseits ist bei OBERREUTER ein unaufhebbarer Gegensatz, der nur in totalitären oder autoritären Staaten sich zugunsten der Macht auflöst. GöRTEMAKER spricht 2002 davon, dass die Notstandsgesetze durch die APO mehr oder weniger instrumentalisiert wurden 
und zu einer verstärkten Mobilisierung führten. KIESINGER als Kanzler der großen Koalition, der seit 1933 NSDAP-Mitglied gewesen war, wurde zuweilen verdächtigt, die erweiterten Kompetenzen der Notstandsgesetzgebung zu missbrauchen (GÖRTEMAKER 2002: 206 f.). Wird der Verteidigungsfall festgestellt, werden eine ganze Reihe von klassischen Freiheitsrechten aufgeweicht und relativiert. Dazu zählt auch das Fehlen einer Verpflichtung, die Bürger von Eingriffen in das Brief-, Post- und Fernmeldegeheimnis informieren zu müssen und das Unterlaufen des in Artikel $19 \mathrm{GG}$ garantierten Rechtsweges macht es dem Einzelnen unmöglich, Eigenkontrolle auszuüben, um sich gegebenenfalls dagegen wehren zu können. Werden die Notstandsgesetze in den festgelegten Situationen angewendet, kann die Freiheit der Bürger konkret eingeschränkt werden. Besonders die Einschränkungen des Brief-, Post- und Fernmelde-geheimnisses und die Möglichkeit, bis zu 4 Tage inhaftiert werden zu können, sind konkrete Bedrohungen der Freiheit.

Bis heute haben sich entsprechende Befürchtungen nicht bewahrheitet, denn die Notstandsgesetzgebung wurde noch nicht in der Praxis angewendet, was sämtliche Einwände gegen dieselbe im Bereich der Theorie belässt. Einzelne Bestimmungen der Notstandsgesetze wie zum Beispiel das Fehlen einer Frist, bei der nach Eintreten des Verteidigungsfalles das Parlament einberufen werden muss (vgl. HESSE/ELLWEIN 1997: 273), werden immer wieder kritisch thematisiert, wobei allerdings kaum Neigung von Seiten der Politik besteht, Veränderungen durchzuführen. Die latente Wirkung der neu eingeführten Bestimmungen lässt sich nur schwer abschätzen: Hat die Ausweitung der Exekutivbefugnisse - JESSE und ElLwEIN sprechen von »der Stunde der Exekutive« (ebd.: 272), dies gilt besonders auch dann, wenn man berücksichtigt, dass entsprechende Rechte zuvor von Außen durch die Allierten ausgeübt 
wurden - zu mehr Sicherheit geführt? Wie sieht es mit der Sicherheit und Freiheit aus, wenn die Notstandsgesetze angewendet werden oder gar der befürchtete Missbrauch der Notstandsgesetze stattfindet?

Diese Fragen lassen sich nur teilweise beantworten. Im Bereich der Äußeren Sicherheit stellen die Exekutivbefugnisse der Regierung tatsächlich eine Aufwertung der Sicherheit dar, schon allein dadurch, dass es politische Strukturen gibt, die im Verteidigungsfall als politisches »Backup « fungieren können und schnelle Entscheidungen potenziell möglich machen können. Für Notfälle sind damit Strukturen und feste politische Abläufe vorhanden, die zur Bewältigung einer Krisensituation Anwendung finden können.

Gleichzeitig haben die potenziellen Freiheitseinschränkungen auch über den Notstand hinaus Auswirkungen. Mit der Veränderung des 10. Artikels wurde eine Art Einfallstor für kommende Eingriffe in die individuelle Freiheit geschaffen. BOMmARIUS (2008) verweist in diesem Zusammenhang auf Abhöraktionen durch den Verfassungsschutz und auf das BKA-Gesetz, das aktuell verabschiedet werden soll und massive Eingriffe in die Privatsphäre (Online-Durchsuchungen) ermöglichen soll und die er als langfristige Folge der Notstandsgesetze sieht. Tatsächlich besteht bei Aufweichungen und Relativierungen von Grundrechten immer die Gefahr, dass diese erst der Anfang sind und dass weitere Einschränkungen von Freiheitsrechten im Anschluss daran leichter fallen und auch dem Missbrauch Tür und Tor geöffnet wird.

Gegen den Missbrauch der Bestimmungen der Notstandsgesetze gibt es einige Vorkehrungen, die allerdings und glücklicherweise ebenfalls noch nicht in der Praxis erprobt werden mussten. Zum einen bleibt das Bundesverfassungsgericht während des gesamten Zeitraums in Funktion und kann uneingeschränkt seine Funktionen wahrnehmen. Zum anderen hat nach 
Art. 20 (4) jeder Bürger zudem das Recht, gegen den Missbrauch vorzugehen: «Gegen jeden, der es unternimmt, diese Ordnung zu beseitigen, haben alle Deutschen das Recht zum Widerstand, wenn andere Abhilfe nicht möglich ist« (Art. 20 (4) GG). Konkret gemeint mit »Ordnung" sind hier die vorangehenden Absätze des Art. 20 in dem die folgenden wichtigen Prinzipien festgelegt sind: Demokratie, Bundesstaatlichkeit, Volkssouveränität und deren Ausübung in Form von Wahlen und Abstimmungen, Bindung der Legislative an die verfassungsmäßige Ordnung, Bindung von Exekutive und Judikative an Gesetz und Recht. Trotz dieser Bestimmungen ist es mehr als fraglich, ob gegen einen möglichen Missbrauch der Notstandsgesetze nur aufgrund dieser Bestimmungen effektiv vorgegangen werden kann. Viel wird in einer solchen Situation des Missbrauchs davon abhängen, wie die Reaktionen der anderen europäischen Staaten ausfallen und wie die politischen und gesellschaftlichen Rahmenbedingungen sind, welche die Situation erst ermöglichten.

\subsection{Terrorismus I - die Rote Armee Fraktion (RAF)}

Nach WALDMANN kann man Terrorismus als "planmäßig vorbereitete, schockierende Gewaltanschläge gegen eine politische Ordnung aus dem Untergrund verstehen « (WALDMANN 2005: 12). Die Auswirkungen solcher Anschläge sind vielschichtig und haben Folgen unterschiedlicher Reichweite für die betreffende Gesellschaft. Die Geschehnisse der RAF-Zeit und die Anschläge vom 11. September 2001 (9/11) sind zwei wichtige Einschnitte für die Entwicklung von Sicherheit und Freiheit in unserer Gesellschaft.

Aufgrund von Parallelen bei den Auswirkungen werden sie im Folgenden als Terrorismus I und II bezeichnet. Die folgenden Abschnitte widmen sich dem Terrorismus des deutschen Herbstes und die Auswirkungen der verschiede- 
nen Ereignisse auf die Ausprägung und die Entwicklung von Sicherheit und Freiheit in Deutschland.

\subsection{1 Überblick}

Die außerparlamentarische Opposition (APO), welche im Jahr der Notstandsgesetze ein wichtiger politischer Akteur gewesen war, zerfiel kurz nach der Verabschiedung der Notstandsgesetze in drei größere Gruppen, die (vgl. GÖRTEMAKER 2002: 210) sich entweder politisch integrierten oder versuchten, sich in alternativen Lebensformen selbst zu verwirklichen (ebd.). Ein dritter und quantitativ marginaler Teil versuchte sich unmittelbar als Terroristen oder sympathisierte zumindest mit ihnen. Auf diese Weise begann die in Deutschland bislang einmalige Karriere der Rote Armee Fraktion (RAF) ${ }^{1}$, die von Beginn der 1970 er Jahre eine Spur der Zerstörung und des Todes durch Deutschland zog. Neben anderen Gruppierungen war sie diejenige, welche die meiste Aufmerksamkeit auf sich zog und den größten Schaden verursachte. Es würde den Rahmen dieser Untersuchung bei weitem sprengen, würde man versuchen, das Phänomen RAF hier umfassend aufzubereiten. Die soziologische Literatur hierzu ist Legende, vor allem auch die Veröffentlichungen von NeIdhardt (u.a. 1982 und 2006). Im Kontext der Studie ist aber relevant, besonders die Entwicklung des Phänomens RAF aufzeigen.

Insgesamt tötete die RAF ab 197134 Menschen (vgl. DietL u.a. 2006: 69), darunter den Generalbundesanwalt SiEgFried BuBACK und den Arbeitgeber-

1 Neben der RAF gab es noch diverse andere Gruppen, die aber wesentlich weniger prominent und teilweise praktisch vergessen wurden, darunter beispielsweise die Tupamaros München (vgl. Sturm 2006: 99 ff.) und andere (vgl. WeInHAUER/REQUATE 2006). 
präsidenten Hans Martin Schleyer (vgl. Kovacic 1987:52), um nur die prominentesten Fälle zu benennen. Das letzte Opfer war ein GSG 9-Beamter, der beim Festnahmeversuch der RAF-Mitglieder GRAMS und HOGEFELD in Bad Kleinen bei einem Schusswechsel mit den Terroristen starb. Des Weiteren gehörten Banküberfälle, Brandstiftungen, Entführungen und Bombenanschläge zum Repertoire der Gruppierung. Begleitet wurden die Aktionen der RAF sehr häufig von Bekennerschreiben (vgl. u.a bei BURDRICH 2005 oder IDVerlag 1997), die Einblicke in die gedankliche Welt der Terroristen ermöglichten. Die Zeit der RAF kann man anhand der verschiedenen Generationen von Terroristen unterteilen (vgl. DIETL u.a. 2006). Die erste Generation bestand im Kern aus Andreas BaAder, Gudrun Ensslin, Ulrike Meinhof, Horst Mahler, Peter Homann, Brigitte Asdonk, die 1971 mit dem Konzept "Stadtguerilla« an die Öffentlichkeit traten und mit der »Organisation von bewaffneten Widerstandsgruppen« (RAF 1971, zitiert aus BURDRICH 2005: 15) begannen.

Die Befreiung ANDREAS BADERS war dabei der spektakuläre Anfang. Im Laufe der Zeit wurde nach verstärkten Fahndungsbemühungen der Polizei ein Großteil der ersten Generation inhaftiert, was dann dazu führte, dass die zweite Generation sich bemühte, ihre inhaftierten Gesinnungsgenossen freizupressen. Die Stockholmer Geiselnahme, sowie die Entführung von HaNS MARTIN SCHLEYER waren markante Aktionen dieser Phase. Als ein Freipressversuch der PLO, welche die Lufthansamaschine Landshut nach Mogadishu entführt hatte, scheiterte, begingen BAADER, RASPE und ENSSLIN im Stammheimer Gefängnis Selbstmord. Die dritte Generation veränderte ihre Zielsetzung und versuchte nun nicht mehr, Inhaftierte frei zu bekommen. Stattdessen wurden gezielte Angriffe durchgeführt, oftmals auch mit Schulterschluss zu 
ausländischen Gruppierungen wie der Action Directe (vgl. RAF-Bekennerschreiben zum Anschlag auf die Rhein-Main Air Base). Da das Führungspersonal und die Logistik der ersten Generationen nicht mehr zur Verfügung standen (DIETL U.a 2006: 78), war dieser Schritt notwendig geworden. Die RAF erklärte sich im Jahre 1998 selbst für aufgelöst, nachdem ihr Ziel, das »politische System durch Terrorakte zu stürzen oder nachhaltig zu erschüttern« (ebd.) nicht erreicht wurde.

\subsubsection{Anti-Terror-Maßnahmen}

Durch den Terrorismus bekommen Delikte, die eigentlich bereits durch geltendes Recht abgedeckt sind, eine neue Konnotation. Es geht dann nicht mehr um kriminelle Straftaten, sondern vielmehr um einen Angriff auf das politische System an sich. Die Situation, die dabei entsteht, hat SCHEERER sehr zutreffend als »symbolischer Belagerungszustand « (SCHEERER 1980: 120) bezeichnet, der sich durch die Phänomene Entscheidungszwang - für oder gegen ein bestimmtes System - und Konsensuszwang auszeichnet. Letzterer meint die Erhöhung des »sozialen, rechtlichen und politischen Risikos« (ebd. 121f.) für das Äußern abweichender Einstellungen bei gleichzeitig sinkender Toleranz. Die Konsequenz sind dabei Anti-Terror-Gesetze, die SCHEERER nach drei Arten (vgl. ebd.: 126ff.) unterscheidet. Unter der Gruppe der so genannten Organisationsgesetze fasst er Regelungen zusammen, welche die Staatsstrukturen umorganisieren, so dass die Handlungsfähigkeiten der Terroristen beeinträchtigt oder unterbunden werden können. Denkbar sind in diesem Zusammenhang Sondergerichte, neue Polizeistrukturen oder Kompetenzverlagerungen. Als Verfahrensgesetze bezeichnet er alle »Veränderungen nicht-materiellen, 
prozessualen Strafrechts (ebd.: 127), die in der Regel darauf ausgerichtet sind, die Befugnisse der Strafverfolger zu erweitern und die Last für Verdächtige und Beschuldigte zu erhöhen. Die dritte Gruppe bezieht sich auf Veränderungen im Strafrecht, in dem neue Strafbestände etabliert oder bestehende Paragraphen verschärft wurden. Die Zeit des RAF-Terrorismus wurde dazu genutzt, in allen genannten Bereichen entsprechende Veränderungen zu installieren. Dies geschah vor allem in der zweiten Generation der RAF, als sich zeigte, dass das Inhaftieren der Gründergeneration der RAF kein Abreißen der Aktivitäten bewirkte. Die verantwortlichen Politiker wurden durch die Ereignisse und die Berichterstattung unter Druck gesetzt: »Wenn es uns nicht gelingt, diesen totalen Angriff der Terroristen auf Staat und Gesellschaft und die Menschen abzuwehren, dann kann man überhaupt die liberale Rechte- und Innenpolitik vergessen« (FDP-Chef MARTIN BANGEMANN 1977, zitiert nach KovaCIC 1987).

Auf der rechtlichen Ebene vollzog sich die Abwehr des Staates in einer Ergänzung des Strafgesetzbuches um die Paragraphen 129a und $130 \mathrm{a}$. Neben den Strafbestand der Bildung einer kriminellen Vereinigung des $\$ 129$ wurde zusätzlich mit §129a die Bildung terroristischen Vereinigungen mit Freiheitsstrafe belegt. Gut ein Jahrzehnt danach wurden weitere Absätze dem Paragraphen hinzu gefügt: Rädelsführer und Hintermänner können nun mit Freiheitsstrafe nicht unter drei Jahren bestraft werden, aber auch das Unterstützen oder Werben für eine terroristische Vereinigung konnte mit sechs Monaten bis zu fünf Jahren Gefängnis bestraft werden. Fälle geringerer Schuld konnten nach $\S 49 \mathrm{StGB}$ milder beurteilt werden, gleichzeitig konnte das Gericht das passive Wahlrecht aberkennen. Neben den Tatbestand der Volksverhetzung nach $\S 130$ StGB wurde die Anleitung zu Straftaten unter Strafe gestellt: 
»Wer eine Schrift (...), die geeignet ist, als Anleitung zu einer in $\S 126$ Abs. 1 genannten rechtswidrigen Tat zu dienen, und nach ihrem Inhalt bestimmt ist, die Bereitschaft anderer zu fördern oder zu wecken, eine solche Tat zu begehen, verbreitet, öffentlich ausstellt, anschlägt, vorführt oder sonst zugänglich macht, wird mit Freiheitsstrafe bis zu drei Jahren oder mit Geldstrafe bestraft«(§130a (1)).

Im gleichen Sinne wird nach §130a (2) StGB auch jeder bestraft, der Anleitungen zu Straftaten nach §126 StGB - Störung des öffentlichen Friedens durch Androhung von Straftaten wie Mord, Totschlag, Völkermord, Raub, räuberische Erpressung usw. - gibt. Im Jahre 1977 wurde während der Entführung Hans MARTIN SCHLEYERS das so genannte Kontaktsperregesetz beschlossen, das gegen diejenigen angewandt werden kann, die nach §129a StGB oder §129b StGB im Gefängnis sind oder für die wegen entsprechender Straftaten Haftbefehl besteht. Konkret heißt es dazu im 31. Paragraphen des Einführungsgesetz zum Gerichtsverfassungsgesetz im 4. Abschnitt unter dem Stichwort Kontaktsperre: »Besteht eine gegenwärtige Gefahr für Leben, Leib oder Freiheit einer Person, begründen bestimmte Tatsachen den Verdacht, daß die Gefahr von einer terroristischen Vereinigung ausgeht, und ist es zur Abwehr dieser Gefahr geboten, jedwede Verbindung von Gefangenen untereinander und mit der Außenwelt einschließlich des schriftlichen und mündlichen Verkehrs mit dem Verteidiger zu unterbrechen, so kann eine entsprechende Feststellung getroffen werden« ( $(31 \mathrm{EGGVG).}$

Auf dieser gesetzlichen Grundlage wurde es den Behörden ermöglicht, die Kommunikation zwischen Anwälten und inhaftierten Mandanten zu unterbrechen, gleichzeitig wurde die Anzahl der zulässigen Verteidiger pro Mandanten auf jeweils einen beschränkt (vgl. JANISCH 2002).

Das Kontaktsperregesetz wurde im parlamentarischen Schnellverfahren innerhalb einer Woche (vgl. SCHEERER 1980: 131) verabschiedet, wobei auch 
die eigentlich beteiligten Ausschüsse des Bundestages unter dem Druck der Ereignisse praktisch außen vor gelassen wurden. Schon kurze Zeit nach Veröffentlichung des Gesetzes im Bundesgesetzblatt verhängte der damalige Innenminister JOCHEN VOGEL mehrere Kontaktsperren über inhaftierte RAFMitglieder.

Zusätzlich wurde als Fahndungsinstrument die Rasterfahndung eingeführt, bei der die Polizei auf Daten anderer Behörden im Rahmen eines Auskunftbegehrens zugreift, um Informationen über unbekannte Täter zu erhalten (vgl. HeRoLD 1985:85). Die rechtliche Grundlage für diese Vorgehensweise ist die Rechts- und Amtshilfe nach Art. $35 \mathrm{GG}$. Zu unterscheiden sind dabei die positive und negative Rasterfahndung. Bei ersterer werden alle betroffenen Datensätze aktiv und gezielt nach gegebenen Merkmalen durchsucht. Bei der negativen Rasterfahndung hingegen werden aus der betroffenen Datei Datensätze, die nicht den Kriterien entsprechen, ausgesiebt beziehungsweise gelöscht, so dass sich durch dieses Ausschlussverfahren die Zahl der Verdächtigen kontinuierlich reduziert (vgl. HEROLD 1985: 94). Als klassischer Anwendungsfall einer negativen Rasterfahndung nannte der ehemalige BKA-Chef HORST HEROLD in einem Spiegelinterview das Ermitteln von konspirativen Wohnungen mit Hilfe eines Datensatzes, der die Zahlungsweise von Stromrechnungen - in diesem Falle die Barzahlung zur Vermeidung von Spuren - mit legalen Namensträgern abglich. Als Abgleichdatensätze dienten dabei unter anderem Autohalter, Rentner, Versicherte usw. (vgl. HentSCHEL/PötZL 1986: 38 ff.).

In jüngerer Vergangenheit wurde die Rasterfahndung als polizeiliches Instrumentarium immer wieder in die öffentliche Diskussion gebracht und zusätzlich die entsprechenden gesetzlichen Regelungen ergänzt und aktualisiert. Als Beispiel für diese rechtliche Entwicklung kann die Novelle des baye- 
rischen Polizeiaufgabengesetzes gelten, die im Jahr 2007 verabschiedet wurde. In der Begründung zu dem Gesetzesentwurf werden die Terroranschläge vom 11. September 2001, die versuchten Anschläge mit Kofferbomben in Dortmund und Koblenz und die Anschläge von London und Madrid aus dem Jahre 2005 als Zeichen für eine grundlegend veränderte Gefährdungslage benannt (vgl. BLDS 15/9460), die zusammen mit der »Organisierten und schweren Kriminalität die Sicherheitsbehörden vor neue Herausforderungen« (ebd.: 1) stelle. Die Lösung für dieses Problem sei eine Konzentration auf Straftatverhinderung und Gefahrenabwehr, in der die präventive Rasterfahndung eine zentrale Rolle spielen soll, da - diese Argumentationsfigur findet sich auch bei der Begründung der akustischen Wohnraumüberwachung - »die eigenen Erkenntnisse der Dienste und der Polizeibehörden allein oft nicht ausreichen, um potentielle Attentäter zu entdecken und drohende Anschläge abzuwehren« (ebd.).

\subsubsection{Bewertung: Freiheit und Sicherheit vor dem Hintergrund der RAF}

Das politische System der Bundesrepublik konnte der Terrorismus der RAF zwar nicht wie geplant stürzen oder nachhaltig erschüttern, auf Sicherheit und Freiheit hatten die oben festgehaltenen Geschehnisse und Maßnahmen durchaus Einfluss. Obwohl rein quantitativ und relativ gesehen die Opfer und Schäden der Terroraktionen gering waren, war der psychologische Einfluss auf die Gesellschaft und die verantwortlichen Politiker enorm. Gleichwohl zeigt das Verbleiben der RAF im Untergrund auch, dass sie den Staat und das System, das sie stürzen wollten, nur punktuell und begrenzt herausfordern konnten. Zurecht bezeichnet WALDMANN die Tätigkeit terroristischer Gruppen ganz all- 
gemein als eine »Art Verlegenheitsstrategie« (WALDMANN 2005: 13), die versucht, über wirksame Setzung von gezielten Schocks zu kompensieren, dass für eine breit angelegte Konfrontation mit dem verhassten System die Ressourcen fehlen. Die dabei eingesetzte Gewalt hat somit einen symbolischen Stellenwert, der bestimmte Botschaften als Teil einer Kommunikationsstrategie übermitteln soll (vgl. ebd.). Wichtig für die Terroristen ist dabei, dass ihre Taten einerseits Aufmerksamkeit erzielen und andererseits Sympathien für ihre Sache wecken. Diese Konstellation sorgt dafür, dass die Bedeutung von Sicherheit drastisch erhöht wird, besonders wenn die Gesellschaft, welche durch die Gewalt des Terrors getroffen wird, ansonsten sehr arm an Gewalt ist. Dieses Durchbrechen des staatlichen Gewaltmonopols wird damit zum Kennzeichen des unmittelbaren Reduzierens von Sicherheit durch den Terrorismus selbst. Neben den Taten der RAF selbst waren vor allem auch die ständigen Fahndungsplakate in der Öffentlichkeit ein immer präsentes Symbol. In der Rückschau kann man sagen, dass die staatliche Vorgehensweise die reduzierte innere Sicherheit, die durch die Taten der RAF entstanden waren, wieder herstellen konnte. Nationaler Terrorismus durch die RAF spielt bei der Produktion von Unsicherheiten in Deutschland heute kaum eine Rolle mehr und wird überlagert von internationalen Terrorismusformen, die seit den Anschlägen vom 11. September 2001 weltweit präsent sind.

Die einzelnen Regelungen wie die neu geschaffenen Paragraphen wurden nur selten eingesetzt, sie blieben aber trotzdem hartnäckig im Strafgesetzbuch bestehen. Die Paragraphen 129 a und 130 a entwickelten sich gewissermaßen zu Gummiparagraphen, die es ermöglichten, eine Vielzahl an Verhaltensweisen darunter zu subsumieren. Vor allem der §129a entwickelte sich zu einer »Art Präventionsstrafnorm« (HOLzBERGER 2000), der letzten Endes eine 
Umkehr der Beweisführung zu Lasten der Beschuldigten bewirkte. Zusätzlich wurde die Mittäterschaft zum zentralen Punkt, der auf Grund der Zugehörigkeit zur RAF über den Umweg der terroristischen Vereinigung zu Verurteilungen führte (vgl. Strö̈BELE 1998). Bedenkt man, dass eine Vielzahl von recht einfachen Verhaltensweisen unter diesen Paragraphen fallen, so kann man diese Regelungen als weitgehende Einschränkungen der Freiheit interpretieren. In ähnlicher Weise wirkte die Rasterfahndung in ihrer verdachtsunabhängigen Form, wobei die technischen Entwicklungen der letzten zwei Jahrzehnte mit ihr zusammenwirken können. Die Entwicklung von leistungsstarken Computern, in Verbindung mit verbesserten Datenbanken ergibt im Zusammenhang mit entsprechenden Suchrastern eine für die Freiheit gefährliche Mischung, weil nun viele verschiedene Merkmale zeitnah rekombiniert werden können. Gerade bei der positiven Rasterfahndung besteht in diesem Sinne die Möglichkeit, zusätzliche Merkmale unbemerkt abzufragen (vgl. HEROLD 1985: 94) und so die Suchkriterien unzulässig und unkontrolliert auszuweiten. Für den einzelnen Bürger bedeutet dies, dass keinerlei Kontrollmöglichkeit mehr für ihn besteht und nicht einzuschätzen ist, welche Daten über ihn erhoben und wie die einzelnen Datensätze kombiniert werden. Es ist ebenfalls nur schwer möglich, Zugriff auf die Daten zu bekommen, um gegebenenfalls ein falsches Datenabbild wieder zu korrigieren.

Die unterschiedliche Architektur der Datenbanken, die in den 80 er Jahren des letzten Jahrhunderts ein Problem darstellte und durch einen Mangel an Kompatibilität effektiv die Daten vor umfassenden Abgleichen schützte, verliert an Bedeutung, da insgesamt bei der Neukonzeption von Datenbanken Schnittstellen zum Abgleich mit eingeplant werden und ganz allgemein der Trend weg von monolithischen Datenbanken hin zu modular aufgebauten, an 
Schnittstellen orientierten Varianten geht. Im folgenden Kapitel werden die technologischen Entwicklungen und ihre Gefahren für die Freiheit noch weitergehend thematisiert.

\subsection{Das Volkszählungsurteil des BVerfG - Das Recht auf informationelle Selbstbestimmung}

Im Jahre 2006 wandte sich das Bundesverfassungsgericht in einem Urteil (BvR 518/02) gegen die präventive Rasterfahndung, wie sie im Polizeigesetz Nordrhein-Westfalens geregelt ist, und stellte sie - unter der Bedingung, dass eine konkrete Gefahr für hochrangige Rechtsgüter nicht gegeben ist - als unvereinbar mit dem Recht auf informationelle Selbstbestimmung dar. Dieses Recht stellt heute eine der wichtigsten Freiheitssicherungen dar, indem es die Menschenwürde und die freie Entfaltung der Persönlichkeit vor dem Hintergrund von Datenschutz und Eigenkontrolle in eine griffige Form bringt. Das Recht selbst beruht auf dem Volkszählungsurteil des Bundesverfassungsgerichts aus dem Jahre 1983 (AZ.1 BvR 209, 269, 362, 420, 440, 484/83). Dieses Urteil stellt einen der wichtigsten Meilensteine bei der Entwicklung der Freiheitsrechte in der Bundesrepublik dar und kann in seiner Bedeutung und Reichweite kaum überschätzt werden.

\subsubsection{Vorgeschichte des Urteils}

Das Volkszählungsgesetz vom 25. März 1982 (VZG 1983) wurde bereits im Vorfeld und nach der Verabschiedung durch den Bundestag auf vielfältige Art und Weise kritisiert. Die Kritik entzündete sich vor allem an dem Umfang an 

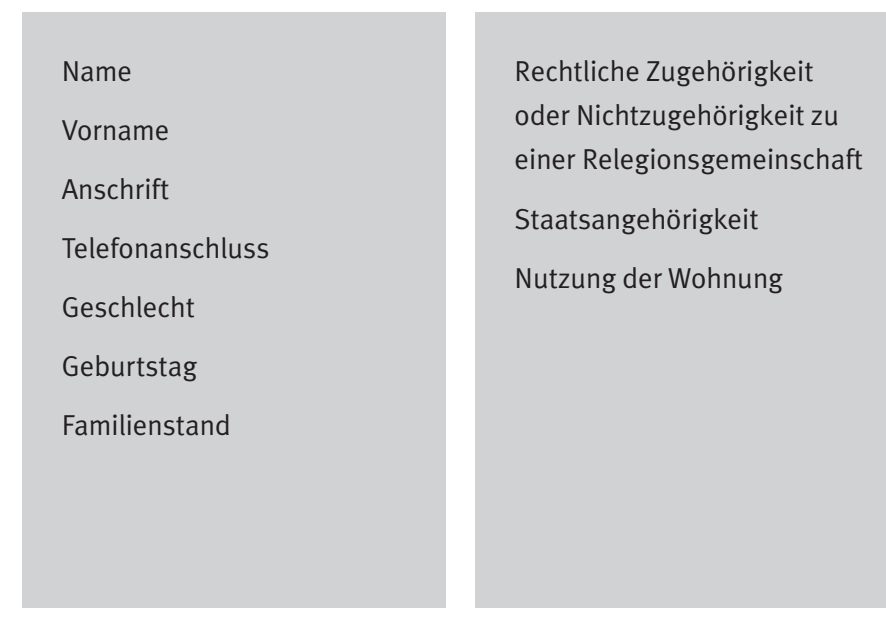

Nutzung der Wohnung

Abbildung 11 Erhobene Informationen der Volkszählung 1983 (abgeändert nach, vgl. VZG 1983; § 2,3)
Quelle des überwiegenden

Lebensunterhalts

Beteiligung am Erwerbsleben

Beruf und Dauer der

Ausbildung

Höchster Schulabschluss

Name und Anschrift der

Ausbildungsstätte

Stellung im Beruf und

ausgeübte Tätigkeit

Merkmalen (siehe Abbildung 11), die bei der Volkszählung abgefragt wurden, sowie an der Weitergabe der Daten an die Meldeämter und nicht zuletzt an der zehnjährigen Aufbewahrungsfrist der Fragebögen (vgl. BORCHERS/WILKENS 2007). Das Gesetz benennt in seinen ersten Paragraphen detailliert die verschiedenen Informationen, die über den einzelnen Bürger gesammt werden sollten (vgl. VZG 1983: §2,3).

Nach $\S 5$ VZG waren alle Volljährigen oder einen eigenen Haushalt führenden Minderjährigen sowie die Wohnungsinhaber auskunftspflichtig. Gegen das Volkszählungsgesetz wurden diverse Klagen eingereicht, die das Gesetz zunächst einstweilig stoppten und dann zu entsprechenden Anpassungen führten. Befürchtet wurden der gläserne Bürger und eine Entwicklung hin zu einem Überwachungsstaat. Das Bundesverfassungsgericht gibt im ersten Abschnitt der Urteilsbegründung sehr anschaulich die Ausgangslage wieder, die zu den Protesten gegen das Gesetz führten: 
»Die durch dieses Gesetz angeordnete Datenerhebung hat Beunruhigung auch in solchen Teilen der Bevölkerung ausgelöst, die als loyale Staatsbürger das Recht und die Pflicht des Staates respektieren, die für rationales und planvolles staatliches Handeln erforderlichen Informationen zu beschaffen. Dies mag teilweise daraus zu erklären sein, daß weithin Unkenntnis über Umfang und Verwendungszwecke der Befragung bestand und daß die Notwendigkeit zur verläßlichen Aufklärung der Auskunftspflichtigen nicht rechtzeitig erkannt worden ist, obwohl sich das allgemeine Bewußtsein durch die Entwicklung der automatisierten Datenverarbeitung seit den Mikrozensus-Erhebungen in den Jahren 1956 bis 1962 (vgl. BVerfGE 27,1) erheblich verändert hatte.

Die Möglichkeiten der modernen Datenverarbeitung sind weithin nur noch für Fachleute durchschaubar und können beim Staatsbürger die Furcht vor einer unkontrollierbaren Persönlichkeitserfassung selbst dann auslösen, wenn der Gesetzgeber lediglich solche Angaben verlangt, die erforderlich und zumutbar sind. Zur Beunruhigung mag nicht zuletzt beigetragen haben, daß auch Sachkundige die Überzeugung vertraten, das Volkszählungsgesetz 1983 genüge trotz einstimmiger Verabschiedung in den gesetzgebenden Körperschaften schon in den Vorschriften über die Erhebung der Daten und vor allem in den Bestimmungen über deren Verwertung nicht hinreichend den verfassungsrechtlichen Anforderungen.« (BverfGE 65, 1 Volkszählung: 2)

Viele Probleme des Datenschutzes entstehen genau aus der angesprochenen Konfliktstellung zwischen einem berechtigten Interesse an einer möglichst genauen und exakten Datenlage, die eine wichtige Bedingung für rationales und erfolgreiches politisches Handeln sein kann und auch sollte, und dem Bedürfnis nach Privatheit der Daten. Letzlich ist diese Konfliktstellung nur durch eine umfassende und glaubwürdige Anonymisierung der Daten abzumildern, wobei dies im Rahmen der Volkszählung kaum möglich erschien, nicht zuletzt weil die Volkszählung zum Teil auch in Form von Interviews bei den Befragten zu Hause stattfinden sollte. Die abgefragten Merkmale der Volkszählung kann man in der Rückschau als überwiegend harmlos einschätzen, da bei fast jeder Anmeldung bei einem beliebigen Dienst auf der Plattform Internet heute wesentlich detaillierte Daten freiwillig preisgegeben 
werden. Das vom Bundesverfassungsgericht festgestellte Unvermögen für Nicht-Fachleute, moderne Datenverarbeitungsmöglichkeiten durchschauen zu können, ist hingegen bis heute aktuell geblieben, wobei heute das Problem nicht in einem Mangel an Information über diese Möglichkeiten besteht: Prinzipiell kann man sich über nahezu Alles Informieren, allerdings bedarf es einiger Fachkompetenz und eines profunden Kenntnisstands bezüglich der Materie, um etwaige Risiken einschätzen zu können.

Auf das Urteil hin wurde die Volkszählung entsprechend umkonzipiert und in den Jahren 1987 und 1988 durchgeführt. Trotz des Urteils entwickelte sich eine Protestbewegung, die zum Boykott der Volkszählung aufrief. APPEL und Hummel registrieren in diesem Zusammenhang zwei Hauptargumentationslinien der Boykotteure. Zum einen wurden die Argumente von 1983 neu aufgegriffen (Kosten, wissenschaftliche Fragwürdigkeit, Mangel an Datenschutz, zunehmende Verflechtung mit anderen Informationstechnologien, Ausbau der Überwachungsapparate des Staates, vgl. ApPel/Hummel 1988: 21). Zum anderen wurde die persönliche Betroffenheit einzelner Bürger, die negative Erfahrungen mit Datenerhebungen und daraus abgeleiteten politischen Entscheidungen gemacht hatten, ein weiterer wichtiger Faktor (vgl. ebd.). Bis 1987 formierten sich aus dem Umfeld der bereits aktiven Volkszählungsgegner, den Betroffenen und weiteren Verbänden (Teile der SPD, Gewerkschaften, Vereinigungen für Datenschutz, usw.) über 1000 Initiativen gegen die Volkszählung (ebd.). Für das Jahr 2011 ist laut Zensusgesetz eine erneute registergestützte Volkszählung geplant, bei der nicht mehr alle Bürger befragt werden sollen (vgl. BRIEGLEB 2008), sondern eine Stichprobe mit Daten der Melderegister und anderer Verwaltungsregister ergänzt werden sollen. 


\subsubsection{Zentrale Bestimmungen des Urteils}

Das Bundeverfassungsgericht ging in seinem Urteil weit über die Problematik der Volkszählung hinaus und formulierte in seiner Urteilsbegründung ein neues Grundrecht, das unmittelbar von der Menschenwürde (Art.1GG) und dem Recht auf freie Entfaltung der Persönlichkeit (Art. 2 GG) ableitet. Die wichtigste Stelle des Urteils lautet wie folgt:

»Mit dem Recht auf informationelle Selbstbestimmung wären eine Gesellschaftsordnung und eine diese ermöglichende Rechtsordnung nicht vereinbar, in der Bürger nicht mehr wissen können, wer was wann und bei welcher Gelegenheit über sie weiß. Wer unsicher ist, ob abweichende Verhaltensweisen jederzeit notiert und als Information dauerhaft gespeichert, verwendet oder weitergegeben werden, wird versuchen, nicht durch solche Verhaltensweisen aufzufallen. [...] Dies würde nicht nur die individuellen Entfaltungschancen des Einzelnen beeinträchtigen, sondern auch das Gemeinwohl, weil Selbstbestimmung eine elementare Funktionsbedingung eines auf Handlungsfähigkeit und Mitwirkungsfähigkeit seiner Bürger begründeten freiheitlichen demokratischen Gemeinwesens ist.« (BverfG 65, 1: 154)

Im Sinne der in dieser Arbeit verwendeten Definition von Freiheit als Eigenkontrolle trifft das Bundesverfassungsgericht hier einen wesentlichen Kern der Thematik, denn

»wer nicht mit hinreichender Sicherheit überschauen kann, welche ihn betreffende Informationen in bestimmten Bereichen seiner sozialen Umwelt bekannt sind, und wer das Wissen möglicher Kommunikationspartner nicht einigermaßen abzuschätzen vermag, kann in seiner Freiheit wesentlich gehemmt werden, aus eigener Selbstbestimmung zu planen oder zu entscheiden« (ebd.).

Wichtig ist dabei, dass nicht nur die Freiheit des Einzelnen von Bedeutung ist, sondern auch das Gemeinwohl (vgl. TINNEFELD u.a. 2005: 146) und die gesamte freiheitliche Grundordnung entscheidend von informationeller Selbstbestimmung eines jeden Bürgers abhängen. Dies betrifft nicht nur die private Kommunikation, sondern wirkt sich auch darüber hinausgehend auf die unmit- 
telbare politische Teilhabe und damit auf die politische Freiheit aller Bürger aus:

»Wer damit rechnet, daß etwa die Teilnahme an einer Versammlung oder einer Bürgerinitiative behördlich registriert wird und daß ihm dadurch Risiken entstehen können, wird möglicherweise auf eine Ausübung seiner entsprechenden Grundrechte (Art. 8, 9GG) verzichten. Dies würde nicht nur die individuellen Entfaltungschancen (...) beeinträchtigen, sondern auch das Gemeinwohl, weil Selbstbestimmung eine elementare Funktionsbedingung eines auf Handlungsfähigkeit und Mitwirkungsfähigkeit seiner Bürger begründeten freiheitlichen demokratischen Gemeinwesens ist« (BverfG 65, 1: 154).

Die Konsequenz und unmittelbar ableitbare Forderung ist umfassender Datenschutz, um die eigene Freiheit und die Freiheit der gesamten Gesellschaft bewahren zu können. Das Recht, Informationen an andere weiterzugeben, muss in diesem Sinne dem Einzelnen überlassen bleiben. Dies gilt ganz besonders auch für Daten, die mit den Mitteln moderner EDV erhoben und weiterverarbeitet werden:

»Hieraus folgt: Freie Entfaltung der Persönlichkeit setzt unter den modernen Bedingungen der Datenverarbeitung den Schutz des Einzelnen gegen unbegrenzte Erhebung, Speicherung, Verwendung und Weitergabe seiner (...) Daten voraus. Dieser Schutz ist daher von dem Grundrecht des Art. 2 Abs. 1 in Verbindung mit Art.1 Abs.1GG umfaßt. Das Grundrecht gewährleistet insoweit die Befugnis des Einzelnen, grundsätzlich selbst über die Preisgabe und Verwendung seiner persönlichen Daten zu bestimmen« (ebd.: 155).

Das Bundesverfassungsgericht weist anschließend (ebd.: 156) darauf hin, dass dieses Recht nicht schrankenlos gilt, da an Informationen, die ein Gesamtbild des Einzelnen und seiner sozialen Realität ergeben, zwangsweise Kommunikationspartner beteiligt sind. Beschränkungen des Rechtes auf informationelle Selbstbestimmung sind daher laut Verfassungsgericht nicht zu vermeiden, allerdings müssen etwaige Einschränkungen auf der Grundlage eines Gesetzes erfolgen und sind nur hinzunehmen, wenn sie »im überwiegenden Allgemeininteresse« (ebd.) liegen. 


\subsubsection{Konsequenzen und Wirkungen des Urteils}

Das Urteil hatte weitreichende Konsequenzen für die weitere Rechtsprechung des Bundesverfassungsgerichts, das bei einer Vielzahl von nachfolgenden Entscheidungen auf das Recht auf informationelle Selbstbestimmung zurück griff (vgl. Abbildung 12). Die hier angeführten wurden dabei aus einer weitaus größeren Liste nach sachlicher Relevanz im Kontext dieser Arbeit ausgewählt, da die genannten zentralen Bestimmungen des Urteils immer

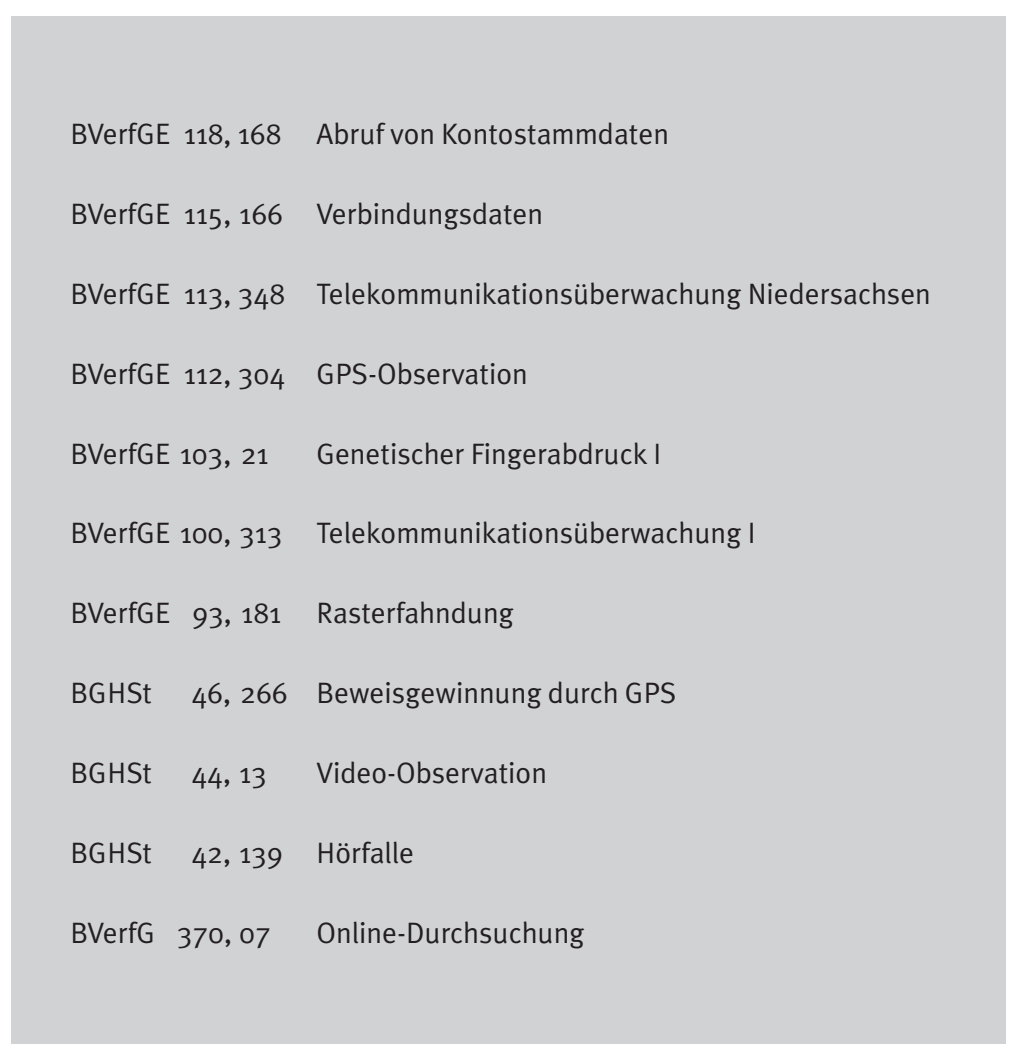

Abbildung 12 Gerichtsentscheidungen mit Bezug auf das Volkszählungsurteil, http://www.servat.unibe.ch/law/dfr/bv065001.html, ergänzt um eigene Daten 
wieder und sehr häufig in anderen Urteilen des Bundesurteils und anderer Gerichte zitiert werden. Es würde an dieser Stelle zu weit führen, alle einzelnen Urteile und ihre jeweiligen Begründungen zusammen zu fassen und en detail aufzubereiten. Trotzdem zeigen die ausgewählten Urteile auch die "Vernetztheit" des Themas mit einer ungeheuren Vielfalt an Inhalten und außerdem auch auf einen schnellen Wandel der Rahmenbedinungen, der seinerseits durch den »informationstechnischen Wandel« (BVerfG 112, 304) bedingt ist: »Wegen des schnellen und für den Grundrechtsschutz riskanten informationstechnischen Wandels muss der Gesetzgeber die technischen Entwicklungen aufmerksam beobachten und notfalls durch ergänzende Rechtssetzung korrigierend eingreifen. (ebd.)«

Das Bundesverfassungsgericht versucht, mit diesem Wandel Schritt zu halten und verweist darauf, dass ebenso wie der Schutzbereich des Fernmeldegeheimnnisses (BVerfG 100, 313, Abs. 162) auch das Grundrecht auf informationelle Selbstbestimmung »entwicklungsoffen« (BVerfG 115, Abs. 166) sei und auf neue Übertragungstechniken im Bereich der Telekommunikation ebenfalls angewendet werde. Darüberhinaus sorgte das Volkszählungsurteil dafür, dass weitere bestimmte Prinzipien zum festen Bestandteil der entsprechenden Urteile wurden. Zunächst einmal gilt jedes persönliche Datum, das erhoben wird, als wichtig: Es gibt keine belanglosen Daten mehr (vgl. BVerfG 118, 168: 88). Die Preisgabe der Daten, die für eine Vielzahl von Zwecken benutzt werden können, muss klar zu erkennen sein für die Betroffenen und muss somit beherrschbar und überschaubar sein (vgl. u.a. Kontostammdatenurteil ebd.). Als ganz besonders schützenswert gilt die Telekommunikation an sich (BVerfG 118, 48, Abs. 79). Verhindert werden soll unbedingt, dass durch die drohende Überwachung es bereits im Vorfeld zu Verhaltensanpassung auf 
Seiten des Bürgers kommt (vgl. Urteil zur Rasterfahndung, BVerfG 92, 181, Abs. 36), der, so beinflusst von den Überwachungs- und Fahndnungsmaßnahmen nun nicht mehr frei handeln kann. In diesem konkreten Fall ist dann nicht nur der Einzelne, sondern das Allgemeinwohl betroffen (vgl. ebd.). Dieser Umstand gilt auch ganz besonders, wenn die entsprechenden Maßnahmen präventiv im Vorfeld von Straftaten stattfinden (vgl. BVerfG 113, 48, Abs. 79).

Das Volkszählungsurteil wird nicht zuletzt auch dann immer zitiert, wenn es darum geht, etwaige Grundrechtseingriffe abzuwägen - laut verschiedener Urteile fordern die Gerichte dann, dass Verdachtsmomente vorliegen müssen, die auf Straftaten von erheblicher Schwere hindeuten und denen zudem mit »normalen« Fahndungsmethoden nicht beizukommen ist (vgl. u.a BVerfG 118, 168; BGHST 44,13; BGHST 42, 139).

Für den Gesetzgeber bedeutete das Volkszählungsurteil, dass künftige Einschränkungen des Rechts auf informationelle Selbstbestimmung und des Datenschutzes »in grundrechtsfreundlichen Bahnen« (TINNEFELD u.a. 2005: 148) verlaufen sollten - die häufige Anrufung der Verfassungsgerichte zeigt aber, dass dies oftmals aber erst eingeklagt werden muss. TINNEFELD fasst in diesem Zusammenhang drei wichtige Prinzipien zusammen: das Prinzip der Normenklarheit, den Grundsatz der Verhältnismäßigkeit und den Grundrechtsschutz (vgl. ebd.: 149). Unter Normenklarheit versteht sie dabei die Bestimmtheit gesetzlicher Regelungen, was heißt, dass der Zweck der einschränkenden Regelung klar definiert sein muss (ebd.). Das Prinzip der Verhältnismäßigkeit meint, dass eine Maßnahme erforderlich und geeignet sein muss, das angestrebte Ziel zu erreichen. In diesem Kontext verweist der Bundesgerichtshof unter Berufung auf das Recht auf informationelle Selbstbestimmung in seinem Urteil zur GPS-Observation und insbesondere bei dem 
Beweisgewinnungsurteil darauf hin, dass besonders aus dem Verbinden und die Kombination unterschiedlicher Überwachungstechniken, die für sich genommen nicht einmal das Recht auf informationelle Selbstbestimung verstoßen, gegen das Prinzip der Verhältnismäßigkeit verstoßen (BGHSt 46, 266, Urteil) werde. Der entsprechende Eingriff in das Grundrecht muss, um diesem Prinzip zu entsprechen, in einem angemessenen Verhältnis zum Eingriffszweck stehen, wobei TINNEFELD davon ausgeht, dass die Größe der tatsächlichen oder angenommenen Gefahrensituation, die zur Rechtfertigung des Eingriffs herangezogen wird, nicht immer klar zu erkennen ist (ebd.: 150). Auf keinen Fall aber ist es mit dem Verfassungsrecht vereinbar, dass ins Blaue hinein Daten im Sinne einer völligen Erfassung erhoben werden.

Für die Freiheit markiert das Errichten des Rechts auf informationelle Selbstbestimmung eine wichtige Zäsur, da erstmalig der Bereich des Privaten ausdrücklich auf die Sphäre der Daten und Informationen ausgeweitet wird. Man kann daher zu Recht von einer Erhöhung des Maßes an Freiheit in Deutschland sprechen. Der neu gewonnene Spielraum sorgt in Verbindung mit den schon existierenden Rechtsweggarantien und Grundrechten dafür, dass dieser Bereich einklagbar und damit für den Einzelnen erreichbar wird. Denn aus der Entscheidung der Verfassungsrichter folgt nicht, dass künftige Gesetze automatisch damit kompatibel wären. Dabei wird wiederum deutlich, wie wichtig das Wissen um Eingriffe in die Freiheit zur Absicherung derselben sind. Politisch-gesellschaftliche Organisationen wie das Bundesverfassungsgericht, benötigt der Einzelne daher dringend zur Verteidigung seiner eigenen Freiheit. Da das Bundesverfassungsgericht nicht von selbst tätig werden kann, ist es wiederum umgekehrt vom persönlichen Engagement einzelner betroffener Kläger abhängig. 


\subsection{Europäische Vereinigungsprozesse}

Wichtige politische Entwicklungen, die Auswirkungen auf den Grad an Sicherheit und Freiheit in Deutschland hatten, verlagerten sich seit Kriegsende weg vom Nationalstaat hin auf die europäische Ebene. Die dort ablaufenden Vereinigungsprozesse veränderten die wirtschaftlichen, sicherheitspolitischen und gesellschaftlichen Rahmenbedinungen, so dass sich völlig neue politische Strukturen ergaben. Schwankend zwischen den Idealen Staatenbund und Bundesstaat verläuft dieser Prozess der Integration »lange Zeit ohne Leitbild« (WEIDENFELD 2002: 10) und orientierte sich sehr lange an wirtschaftlichen Entwicklungen, zu denen sich leichter Kompromisse finden ließen als zu Fragen der politischen Integration oder einer gemeinsamen Verfassung. Die deutsche Vereinigung soll im Folgenden ebenfalls in diesen Integrationsprozess eingeordnet werden.

\subsubsection{Deutsche Vereinigung}

Die Ur-Präambel des Grundgesetzes nennt als letztes Ziel für das gesamte Deutsche Volk die Vollendung der deutschen Einheit. Seit der Wiedervereinigung vom 3. Oktober 1990 ist dieses Ziel erreicht und in diesem Sinne spricht die aktuelle Präambel des Grundgesetzes heute von der Vollendung von Einheit und Freiheit in freier Selbstbestimmung. Diese Einheit vollzog sich über einen Anschluss der ehemaligen DDR und wurde durch die Kooperationsbereitschaft der vier Siegermächte ermöglicht. Letztlich ausgelöst wurde der Einigungsprozess durch die Massenflucht von DDR-Bürgern über die deutschen Botschaften in den Ostblockstaaten und durch Massendemonstrationen - zu den wichtigsten zählten dabei die so genannten Montagsdemonstrationen, bei 
denen unter dem Ruf »Wir sind das Volk« politische Mitspracherechte massiv eingefordert wurden. Nach dem Rücktritt HONECKERS, dem Mauerfall und der nicht mehr kontrollierbaren Ausreise von DDR-Bürgern traten die Gebiete der nun ehemaligen DDR auf der Grundlage des alten Artikel 23 GG der Bundesrepublik Deutschland bei (vgl. EinigVtr 1990: Art.1). Bewältigt wurden dabei große innenpolitische Veränderungen wie die Einführung der DM als neue Währung im gesamten Gebiet (vgl. WAIGEL/SCHELL 1997: 9), die Neustrukturierung des Bundesgebietes und die Anpassung des gesamten Rechtssystems. Zentrale Machtinstrumente der Staatsführung wurden zerschlagen (vgl. WeISS 2001: 222), die SED wurde zur PDS und damit Teil der gesamtdeutschen Parteienlandschaft. Die Einigung Deutschlands fand zeitgleich vom Ende des Kalten Kriegs statt, der in Form dramatischer Änderungsprozesse in allen Staaten des Ostblocks stattfand. Demokratische Reformen, die Öffnung nach Westen und marktwirtschaftliche Umstrukturierungen waren in ganz Osteuropa die Folge. Die anfängliche Euphorie der Ereignisse der Wiedervereinigung wurde überlagert durch Alltagserfahrungen. Vor allem die gewohnte Welt der Ostdeutschen wurde dramatisch verändert:

\footnotetext{
»Mit Mauerfall und Währungsunion auf den Gipfel der Euphorie katapultiert, geriet zunächst der halbe Osten in eine Art Ausnahmezustand: nach den Tränen der Freude und Fassungslosigkeit, den leidenschaftlichen Montagsdemos und den ersten Erkundungsfahrten gen Westen stürzte die Republik der Trabis und Kittelschürzen in einen Kaufrausch, in dem die Freßwelle nahtlos in die Möbel- und PKW-Welle überging. Viele waren benommen vom Ende ihres Haftdaseins. Ihr Glücksgefühl mündete in die schwindelerregende Kreditaufnahme. Die Begeisterung über die liebevoll aufgemachte Postwurfsendung, auf der sogar der eigene Name prangte, war so groß wie die Arglosigkeit, mit der mancher sich windige Versicherungen aufschwatzen ließ. Die Reisebüros verzeichneten Rekordumsätze. Kein Rausch hält ewig. Die Katerstimmung setzte ein, als das Ausmaß, in dem Arbeitsplätze wegbrachen, die Mehrheit ostdeutscher Familien erreicht hatte« (KLIER 2001: 169).
} 
In der Rückschau lässt sich vieles kritisch bezüglich der politischen Entwicklungen nach dem Mauerfall einwenden, zum Beispiel hätte es sich gerade aus wirtschaftspolitischer Sicht gelohnt, gerade die wirtschaftlichen Strukturen und die Arbeitsplätze in der ehemaligen DDR vor dem marktwirtschaftlichen West-Schock zu schützen. Man hatte allerdings im Vorfeld keinerlei Erfahrungen, wie man eine solche politische Aufgaben bewältigen kann und in gleichem Maße ließ das geradezu atemberaubende Tempo der Vereinigung, die sich im Grunde innerhalb eines Jahres unter Einbeziehung der 4 Siegermächte vollzog, auch kaum Raum für politisches Manöverieren. Gerade aber im Bereich des Verfassungsrechtes wurde mit der Integration der DDR über den Beitrittsartikel des Grundgesetzes durchaus die Chance verpasst, die Vereinigung der beiden Staaten durch das Schaffen einer gemeinsamen Verfassung zu vollziehen - stattdessen kann man mit den Worten Albrechts durchaus von einer »Abwicklung der DDR« (ALBRECHT 1992) sprechen.

Bundesdeutsche Normalität, auf der Grundlage von »nicht krisenfreier Marktwirtschaft und einer demokratischen Ordnung, deren Kernbestand nur durch den Freiheitswillen seiner Bürger gesichert werden kann« (STURM 2003: 116), stellte sich mit der Wiedervereinigung ein. Nach der Phase des Umsturzes und der friedlichen Revolution etablierte sich nach der Ankunft der Ostdeutschen die »Normalität des demokratischen Werte- und Meinungsstreites« (ebd.).

\subsubsection{Europäische Integration}

Nach dem Beitritt der DDR zur Bundesrepublik Deutschland wurde der alte Artikel 23 aufgehoben und wurde zum Europaartikel umformuliert. Dabei 
wurde das föderale Element herausgehoben und betont. Über den Bundesrat wirken nun die Bundesländer bei Angelegenheiten der europäischen Union mit. Er kann über Stellungnahmen bei Themen, welche die Bundesländer betreffen, Einfluss nehmen. Die Bedeutung dieser Mitwirkung kann kaum überschätzt werden, da die europäische Ebene im Laufe der Jahrzehnte politisch immer mehr an Bedeutung gewann.

Die Motivation für die Errichtung einer europäischen politischen Gemeinschaft resultierte aus den Erfahrungen des Zweiten Weltkrieges mit seinen vielen Toten und den gewaltigen Zerstörungen. WOYKE nennt in diesem Zusammenhang fünf zentrale Impulse, die das überkommene nationalstaatliche Ordnungssystem überwinden sollten: Überwindung des Nationalismus als Quelle zahlreicher Kriege, neue überstaatliche Sicherheitsstrukturen, Lösung der deutschen Frage, Errichtung eines größeren Wirtschaftsraums, Behauptung zwischen den Hegemonialmächten USA und Sowjetunion ( $\mathrm{vgl}$. WOYKE 1997: 9). Diese Entwicklung startete in der Nachkriegszeit über langfristig angelegte multilaterale Verträge, vor allem zwischen Frankreich, Deutschland, Italien und den Beneluxstaaten. Erste Schritte waren die Europäische Gemeinschaft für Kohle und Stahl (EGKS), die Europäische Wirtschaftsgemeinschaft (EWG) und die EURATOM. Im Laufe der Zeit wurde aus der EWG die EG und diverse verschiedene Länder traten kontinuierlich der Gemeinschaft, die heute 27 Staaten umfasst, bei. Die wichtigsten politischen Einrichtungen sind, ausgehend von den römischen Verträgen von 1957 nach den Verträgen von Maastricht, Amsterdam und Nizza und der Gründung der Europäischen Gemeinschaften, heute der Rat, der Europäische Rat, die Kommission, das Europäische Parlament und der Gerichtshof. Das Verhältnis der einzelnen Organe untereinander ist nicht bestimmt vom Prinzip der Gewal- 
tenteilung, trotzdem besteht eine Art institutionelles Gleichgewicht (vgl. BOLDT 1995: 55). Alle entscheidenden alltäglichen politischen Entscheidungen werden vom Rat gefällt, der im Grunde eine Fachministerkonferenz der Mitgliedsstaaten ist, die in wechselnder Besetzung Themen aus ihren Ressorts entscheiden. Der Europäische Rat dagegen ist das Gipfeltreffen der Staatsund Regierungschefs, der sich den richtungsweisenden politischen Zielsetzungen widmet und für diverse wichtige Politikbereiche die Leitlinien vorgibt (ebd.: 58). Die Kommission übernimmt letztlich die Planung und Überwachung der politischen Entscheidungen, als wichtigstes Kennzeichen hat sie dabei das »Initiativ-monopol« (ebd.) inne, sie dominiert damit den Prozess der Politikplanung und -steuerung. Das Europäische Parlament funktioniert innerhalb dieses politischen Systems nicht wie ein klassisches Parlament, obwohl seit dem Maastrichter Vertrag einige Kontrollrechte - »Ernennung der Kommission, Misstrauensantrag gegen die Kommission, parlamentarische Anfragen und Untersuchungsausschüsse« (NASE U.a. 2003: 34) - und die Entscheidung über den Haushalt zur Verfügung stehen. Es wirkt ebenfalls im Gesetzgebungsverfahren mit.

Die Politik der europäischen Union ruht auf drei Säulen (siehe Abbildung 13), die wichtige Politikfelder repräsentieren: Im Bereich der Europäischen Gemeinschaften handelt die Europäische Gemeinschaft eigenständig und gibt Vorgaben für die Mitgliedsstaaten im Bereich Agrarpolitik, Außenhandel, Verkehr, Wirtschaft- und Beschäftigung, Umwelt, Verbraucherschutz, Asyl und Einwanderungspolitik. Die zweite Säule repräsentiert die Zusammenarbeit zwischen den einzelnen Staaten in Fragen der Außen- und Sicherheitspolitik, um ein möglichst koordiniertes gemeinsames Vorgehen zu ermöglichen. Die dritte Säule steht für die zwischenstaatliche Zusammenarbeit in den 


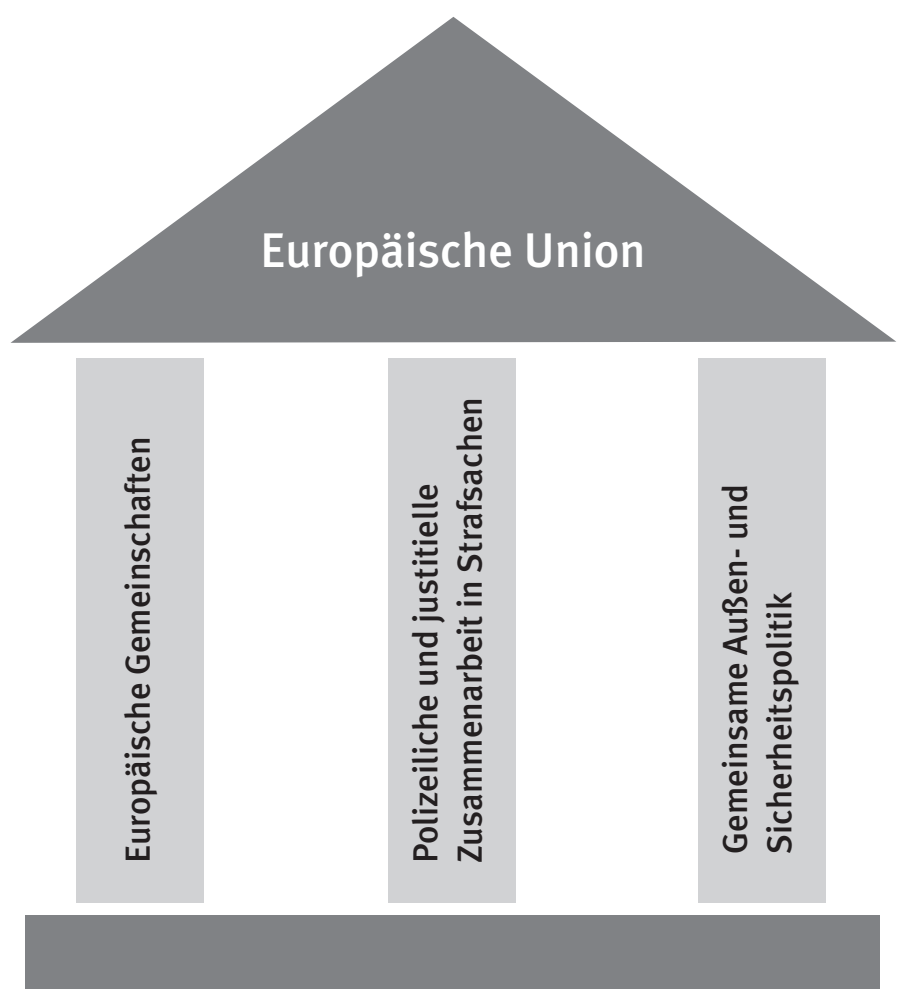

Abbildung 13

Politik der Europäischen Union

Bereichen Polizei und Justiz vor allem im Kampf gegen grenzüberschreitende Kriminalität.

\subsubsection{Bewertung}

Die europäischen Einigungsprozesse hatten enorme Auswirkungen auf die Freiheit der Menschen. Schon der EWG-Vertrag aus dem Jahre 1957 sprach von vier Freiheiten, die im Laufe der Zeit realisiert werden sollten: Freiheit des Warentausches, Freizügigkeit der Arbeitskräfte, die Niederlassungs- und Dienstleistungsfreiheit und die Freiheit des Zahlungs- und Kapitalverkehrs (vgl. THIEL 
1960: 60). All diese Ziele wurden im Laufe der Jahrzehnte umgesetzt und mithin der Grad an Freiheit ganz klar erhöht. Heute ist es möglich, ohne Grenzkontrollen innerhalb des Schengenraumes der EU zu verreisen, in den meisten Ländern kann man zudem mit dem Euro als gemeinsamer Währung bezahlen. Der Abbau der Zollschranken führte zu einem riesigen wirtschaftlichen Binnenmarkt mit großem Nutzen für die europäischen Unternehmen. Begleitet wurden diese Entwicklungen von einer fast schon einmaligen Geschichte des Friedens für die beteiligten europäischen Länder. Die Integration der ehemaligen Ostblockstaaten in die stetig wachsende Union macht die positiven Aspekte deutlicher sichtbar. Der Handel mit diesen Staaten wurde ausgebaut, zudem wirkten die im Rahmen der Osterweiterung gezahlten Ausgleichszahlungen »konjunkturbelebend« (Bertelsmannstiftung 1998: 8). Für den Bereich der Sicherheit ergeben sich parallel dazu ebenfalls positive Effekte. Die offenen Grenzen wirken vertrauensbildend und ermöglichen soziale Kontakte zu anderen Ländern (vgl. ebd.: 9). Gleichzeitig sorgt der gemeinsame Marktzugang nach ge-meinsamen Regeln und das Ausbilden stabiler zwischenstaatlicher Strukturen für eine Festigung zwischenstaatlicher Abläufe. Verlässlichkeit, Berechenbarkeit und Stabilität sind die positiven Folgen.

WOHLFELD fasst in ihrer Analyse die verschiedenen Faktoren von Sicherheit zusammen: Unter weicher Sicherheit versteht sie politische und wirtschaftliche Stabilität, gleichzeitig benennt sie neue Risiken innerer Sicherheit, darunter Organisierte Kriminalität, Illegale Einwanderung und Umweltprobleme. Harte Sicherheit hingegen umfasst territoriale Selbstverteidigung, kollektive Verteidigung und so genannte implizite Garantien (vgl. WoHLFELD 1998: 43 ff.). Für den Bereich der inneren Sicherheit bedeuten die Entwicklungen auf europäischer Ebene eine Aufweichung der strikten Trennung von innerer und äußerer Sicher- 
heit (vgl. Glaessner/LoRenz 2000: 8). Ebenso wie die frei reisenden Bürger wird die Kriminalität als grenzüberschreitend betrachtet und der Wegfall der Grenzkontrollen stellte in diesem Sinne eine Reduzierung (vgl. Buкоw 2000: 48) des Grades an Sicherheit: »Auch in einem Europa ohne Grenzen dürfen Freiräume für reisende Rechtsbrecher nicht hingenommen werden« (SPÖRL 1997: 219). Um dies zu organisieren, wurde in den Polizeigesetzen der Länder die Schleierfahndung als »verdachts- und ereignisunabhängige« (PRANTL 2004: 3) Personenkontrolle eingerichtet. Polizei und Bundespolizei wird damit ermöglicht, ohne vorherigen Verdacht und weiteren Anlass Bürger zu kontrollieren (BESTE 2003: 289). Die Auswahl der kontrollierten Personen erfolgt im wesentlichen intuitiv (vgl. HERNKIND 2000: 192). Die personalintensive Schleierfahndung erbrachte rein quantitativ positive Ergebnisse, wobei zum großen Teil Bagatelldelikte erfasst (ebd.: 194) wurden. Was in diesem Zusammenhang fehlt, ist eine Aufwand-Erfolgs-Analyse, die offenlegen könnte, ob die Ergebnisse der Schleierfahnung die eingesetzen finanziellen und personellen Mittel rechtfertigen. Nur auf diese Weise ließe sich prüfen, ob ihre Anwendung tatsächlich ein Mehr an Sicherheit zur Folge hat.

Der Bayerische Verfassungsgerichtshof setzte der Schleierfahndung 2006 klare Grenzen (vgl. SCHNEIDER 2006: 40) und reduzierte die Kontrollen auf Passkontrollen, da nicht automatisch eine Durchsuchung des Fahrzeugs gerechtfertigt sei. Insgesamt ist die Schleierfahndung ein Schritt dahin, Kontrollen präventiv durchzuführen und nicht in Reaktion auf eine Straftat. Im Rahmen der europäischen Einigungsprozesse erhielten die Behörden zudem auch Zugriff auf das Informationssystem SIS, das als reine Fahndungsdatei eingerichtet wurde. Das Europol-Informationssystem (EIS), das noch mehr Merkmale als SIS speichert, wurde ergänzend eingeführt, allerdings muss das 
jeweils gültige Datenschutzrecht (vgl. Tinnefeld 2005:136) Anwendung finden. In der Gesamtschau der europäischen Entwicklung überwiegt der parallele Zuwachs an Sicherheit und Freiheit für die beteiligten Gesellschaften, obwohl einzelne Entwicklungen auch kritisch zu sehen sind. Dies gilt beispielsweise für die erwähnte Schleierfahndung und für die Informationssysteme, aber auch für politisch-strukturelle Schwächen wie das oft beklagte Demokratiedefizit der Union (Dominanz des EU-Rechts, schwaches Europaparlament, Dominanz der Exekutive durch den Rat, Kompetenzverluste für die Landtage der deutschen Bundesländer bei gleichzeitiger Aufwertung der Bundeslandexekutiven durch die Mitgestaltungsmöglichkeiten des Bundesrates über den neuen Artikel $23 \mathrm{GG}$ ).

Doch all diese kritischen Punkte sind fortdauernden Veränderungen im Prozess der europäischen Einigung und vielfältigen nationalen Einflüssen unterworfen. Umso bedeutsamer sind die erreichten positiven Entwicklungen. Eine Einschätzung der deutschen Wiedervereinigung in puncto Sicherheit und Freiheit im Rahmen dieser Arbeit muss mit der Problematik zurecht kommen, dass die interne Entwicklung in der DDR nicht parallel mit untersucht wurde. Trotzdem ist davon auszugehen, dass für die Bürger der DDR sich der Grad an Freiheit erhöht hat, da die kommunistische Diktatur (und damit z. B. auch die Spitzeltätigkeit der Staatssicherheit) überwunden werden konnte. Besonders die aktive Rolle der DDR-Bürgerrechtler machte deutlich, dass politische Freiheit auch aktiv praktiziert werden kann und muss. Es bleibt abzuwarten und zu hoffen, dass dieses Erleben von aktiver Freiheitsgestaltung zum Teil der politischen Kultur Gesamtdeutschlands werden kann. Für den Bereich der inneren Sicherheit kann man davon ausgehen, dass in der unmittelbaren Umsturzphase die Verhältnisse unsicherer wurden. Mittelfristig galt das auch 
für den Bereich der sozialen und wirtschaftlichen Sicherheit. Die weiteren Entwicklungen in Deutschland beziehen sich auf den gesamten Bereich der deutschen Gesellschaft und betrafen sie als Ganzes.

\subsection{Der große Lauschangriff}

Die alte Fassung des Artikels 13 schützte die Wohnung als unverletzlichen privaten Bereich, in den nur rein physisch in Form einer Durchsuchung eingegriffen werden konnte. Die Bedingungen für solche Eingriffe waren entweder eine richterliche Anordnung (Durchsuchungsbefehl) oder die so genannte Gefahr im Verzug. Weitere Eingriffe durften nur auf Grundlage eines Gesetzes oder unter bestimmten definierten Konditionen erfolgen:

»(1) Die Wohnung ist unverletzlich.

(2) Durchsuchungen dürfen nur durch den Richter, bei Gefahr im Verzuge auch durch die in den Gesetzen vorgesehenen anderen Organe angeordnet und nur in der dort vorgeschriebenen Form durchgeführt werden.

(3) Eingriffe und Beschränkungen dürfen im übrigen nur zur Abwehr einer gemeinen Gefahr oder einer Lebensgefahr für einzelne Personen, auf Grund eines Gesetzes auch zur Verhütung dringender Gefahren für die öffentliche Sicherheit und Ordnung, insbesondere zur Behebung der Raumnot, zur Bekämpfung von Seuchengefahr oder zum Schutze gefährdeter Jugendlicher vorgenommen werden.« (Art. $13 \mathrm{GG}$, alte Fassung)

\subsubsection{Die wichtigsten Bestimmungen}

Mit der Einführung des Großen Lauschangriffs wurde der dreizehnte Artikel des Grundgesetzes im Jahre 1998 durch ein Änderungsgesetz (vgl. BTDS 13/8650) modifiziert und um drei weitere Absätze ergänzt, so dass der prägnant formulierte und schlanke Verfassungsartikel aufgebläht wurde: 
»(1) Die Wohnung ist unverletzlich.

(2) Durchsuchungen dürfen nur durch den Richter, bei Gefahr im Verzuge auch durch die in den Gesetzen vorgesehenen anderen Organe angeordnet und nur in der dort vorgeschriebenen Form durchgeführt werden.

(3) Begründen bestimmte Tatsachen den Verdacht, daß jemand eine durch Gesetz einzeln bestimmte besonders schwere Straftat begangen hat, so dürfen zur Verfolgung der Tat auf Grund richterlicher Anordnung technische Mittel zur akustischen Überwachung von Wohnungen, in denen der Beschuldigte sich vermutlich aufhält, eingesetzt werden, wenn die Erforschung des Sachverhalts auf andere Weise unverhältnismäßig erschwert oder aussichtslos wäre. Die Maßnahme ist zu befristen. Die Anordnung erfolgt durch einen mit drei Richtern besetzten Spruchkörper. Bei Gefahr im Verzuge kann sie auch durch einen einzelnen Richter getroffen werden.

(4) Zur Abwehr dringender Gefahren für die öffentliche Sicherheit, insbesondere einer gemeinen Gefahr oder einer Lebensgefahr, dürfen technische Mittel zur Überwachung von Wohnungen nur auf Grund richterlicher Anordnung eingesetzt werden. Bei Gefahr im Verzuge kann die Maßnahme auch durch eine andere gesetzlich bestimmte Stelle angeordnet werden; eine richterliche Entscheidung ist unverzüglich nachzuholen.

(5) Sind technische Mittel ausschließlich zum Schutze der bei einem Einsatz in Wohnungen tätigen Personen vorgesehen, kann die Maßnahme durch eine gesetzlich bestimmte Stelle angeordnet werden. Eine anderweitige Verwertung der hierbei erlangten Erkenntnisse ist nur zum Zwecke der Strafverfolgung oder der Gefahrenabwehr und nur zulässig, wenn zuvor die Rechtmäßigkeit der Maßnahme richterlich festgestellt ist; bei Gefahr im Verzuge ist die richterliche Entscheidung unverzüglich nachzuholen.

(6) Die Bundesregierung unterrichtet den Bundestag jährlich über den nach Absatz 3 sowie über den im Zuständigkeitsbereich des Bundes nach Absatz 4 und, soweit richterlich überprüfungsbedürftig, nach Absatz 5 erfolgten Einsatz technischer Mittel. Ein vom Bundestag gewähltes Gremium übt auf der Grundlage dieses Berichts die parlamentarische Kontrolle aus. Die Länder gewährleisten eine gleichwertige parlamentarische Kontrolle.

(7) Eingriffe und Beschränkungen dürfen im übrigen nur zur Abwehr einer gemeinen Gefahr oder einer Lebensgefahr für einzelne Personen, auf Grund eines Gesetzes auch zur Verhütung dringender Gefahren für die öffentliche Sicherheit und Ordnung, insbesondere zur Behebung der Raumnot, zur Bekämpfung von Seuchengefahr oder zum Schutze gefährdeter Jugendlicher vorgenommen werden." (Art.13GG, aktuelle Fassung) 
Der Gesetzesentwurf von CDU/CSU, SPD und F.D.P nannte als Problemstellung in dem Gesetzesentwurf das »Interesse (an) einer wirksamen Bekämpfung insbesondere der Organisierten Kriminalität« (BTDS 13/8650: 1) durch die sakustische Überwachung von Wohnungen zum Zwecke der Strafvefolgung « (ebd.) und auch zum Zwecke der »Gefahrenabwehr« (ebd.). Für die erwähnte Form der Überwachung, so der Entwurf weiter, müssten entsprechende verfassungsrechtliche Grundlagen geschaffen werden. In der ausführlichen Begründung des Gesetzesentwurfs wird die spezielle Situation gerade im Umgang mit Organisierter Kriminalität dargestellt. Die Rede ist von kriminellen Strukturen, denen mit dem herkömmlichen Instrumentarien der Strafverfolgung nicht beizukommen sei: Vor allem die Tatsache, dass die Strukturen der Organisierten Kriminalität sich abschotten, wird als Grund genannt, warum beispielweise das Einschleusen von V-Leuten kein adäquater Ersatz für die akustischen Wohnraumüberwachungen sei, zumal auf Grund der im Umfeld gegebenen Gewaltbereitschaft jeder personelle Einsatz erhebliche Risiken berge (vgl. ebd.: 4). Grundsätzlich sei die Überwachung immer nur dann angemessen, wenn ein tatsachenbegründeter Anfangsverdacht besteht und wenn der Einsatz andere Ermittlungsinstrumente »unverhältnismäßig erschwert oder aussichtslos« (ebd.) wäre.

Besieht man sich die einzelnen Erweiterungen des neuen Artikels 13 näher, so wird deutlich, welche weitreichende Wirkung diese für den Einzelnen haben. Die in Absatz 3 angesprochenen technischen Mittel machen es nun möglich, dass Ermittler im Verdachtsfalle die Wohnungen der Verdächtigen von außen und unbemerkt abhören. Jedes gesprochene Wort war damit der Privatheit vollständig entzogen, Im Grunde musste ab da jeder Bürger fürchten, dass das staatliche Ohr mitlauscht und die gewonnenen Erkenntnisse entsprechend 
ermittlungstechnisch ausgewertet werden. Die in Absatz 1 angesprochene Unverletzlichkeit der Wohnung war damit im Grunde obsolet geworden oder umgekehrt betrachtet - hatte sich die Situation für den Bürger noch mehr verschlechtert, da schon zuvor Wohnungen von der Polizei im Rahmen einer Hausdurchsuchung durchsucht werden konnten. Neu ist nun aber, dass der Zugriff nicht mehr offen geschieht, sondern verdeckt. Es besteht damit zunächst keine Möglichkeit für den Einzelnen, sich gegen die Überwachung und die Auswertung in irgendeiner Form zu wehren.

Neben der Veränderung des Grundgesetzes wurde ergänzend die Strafprozessordnung (Art 100a-101 StPO) angepasst (Gesetz zur Verbesserung der Bekämpfung der Organisierten Kriminalität), um die exakten rechtlichen Voraussetzungen für die Überwachung rechtlich zu fixieren.

\subsubsection{Folgen der Grundgesetzänderung}

Im Vorfeld des legislativen Prozesses wurde vom kleinen Koalitionspartner FDP eine Mitgliederbefragung durchgeführt, welche die Zustimmung zu dem Gesetzentwurf ausloten sollte. Im Zuge der Zustimmung innerhalb der Partei trat die amtierende Justizministerin SABINE LEUTHEUSSER-SCHNARRENBERGER, deren Ressort eigentlich federführend tätig sein sollte, nachdem sie schon in den vorangegangen Jahren gegen entsprechende Gesetzesinitiativen vorgegangen war, als Reaktion über die positive Haltung ihrer Partei von ihrem Amt zurück. Die FDP-Politiker Leutheusser-SchnARREnBerger, Baum und HIRSCH legten nach Verabschiedung des Gesetzes Verfassungsbeschwerde vor dem Bundesverfassungsgericht ein, das 2004 der Beschwerde teilweise entsprach. Die Richter sprachen in den Leitsätzen des Urteils von der zwin- 
gend notwendigen Anerkennung eines "absolut geschützten Kernbereichs privater Lebensgestaltung" (BvR 2378/98), in den die akustische Überwachung zu Zwecken der Strafverfolgung nicht eingreifen darf. Die Veränderung des Artikels $13 \mathrm{GG}$ wurde vom Verfassungsgericht als verfassungsgemäß eingestuft (ebd.: 103), sowohl in formaler als auch materieller Hinsicht. Allerdings müssen Vorkehrungen getroffen werden, die sicherstellen, dass die Privatheit und damit die Menschenwürde durch die Abhörmaßnahme nicht beeinträchtigt wird (vgl. ebd.: 137 f.). Wenn also Familienangehörige und engste Vertraute in der Wohnung anwesend sind, so müssen Abhörmaßnahmen unterbleiben. Die Lauscher müssen sich deshalb auch an den betroffenen Räumlichkeiten orientieren. Während Geschäftsräume nicht zum Kernbereich privater Lebensgestaltung gehören (vgl. ebd.: 142), ist in Privaträumen das Abhören auf "strafverfahrensrelevante Inhalte« (ebd.: 149) zu beschränken. Erkenntnisse, die in diesem Sinne illegal erworben werden, unterliegen einem Verwertungsverbot (vgl. ebd.: 178, 184) und müssen gelöscht (ebd.: 186) werden.

Die Bestimmungen der Strafprozessordnung wurden danach überarbeitet und an die Vorgaben des Bundesverfassungsgerichts angepasst. Überwacht werden darf demnach, wer verdächtigt wird, bestimmte schwere Straftaten wie Mord und Totschlag, Raub und Erpressung, Geldwäsche, Kinder- und Jugendpornographie, bestimmte Verstöße gegen das Betäubungsmittel- und Asylverfahrensgesetz, Völkermord, Verstöße gegen das Gesetz über die Kontrolle von Kriegswaffen usw. (vgl. §100 StPO) begangen zu haben. Die Tat muss im Einzelfall schwer wiegen und Verfolgungsalternativen müssen erschwert oder sogar aussichtslos sein. Sowohl die Telekommunikation (vgl. §100 a StPO) als auch der Wohnraum dürfen akustisch überwacht werden, zusätzlich dürfen 
auch außerhalb von Wohnungen Bildaufnahmen der Überwachten hergestellt (vgl. §100h StPO) und Daten und Standorte von »Mobilfunkendgeräten« (§100i StPO) ermittelt werden. Nach Abschluss der Maßnahmen muss unter definierten Voraussetzungen die Überwachung den jeweiligen Betroffenen mitgeteilt werden.

\subsubsection{Bewertung}

In puncto Sicherheit beginnen für Strafverfolgungsbehörden bei der akustischen Überwachung paradiesische Zustände, solange die Maßnahme unerkannt bleibt. Informationen über kriminelle Tätigkeiten sind wichtig für die Strafverfolgung und auch für Straftatprävention. Besonders bei schweren kriminellen Fällen und ganz besonders bei Terrorgefahr besteht ein dringendes Bedürfnis nach Informationen aus erster Hand. Problematisch ist dabei der hohe Einsatz an Personal und finanziellen Mitteln, die bei der Überwachung und der adäquaten Analyse der Gespräche benötigt werden. Die Hürden, die zudem das Verfassungsgericht stellte, sind für die Behörden kaum zu bewältigen. Vor allem das Aussetzen der Überwachung, sobald private Themen zur Sprache kommen, stellt ein Problem dar. Wann weiß der Lauscher, dass er wieder einschalten kann, wann weiß er, dass es jetzt nicht mehr um ein privates Gespräch, sondern um die Planung eines Terroranschlages geht? Realiter ist diese Problematik nicht zu bewältigen, stattdessen muss man davon ausgehen, dass die Lauscher trotzdem auch diese privaten Gespräche abhören.

Für die Freiheit im Sinne von Privatheit war die Einführung des Großen Lauschangriffes ein drastischer Rückschritt. Gefährdet wird sie nicht nur durch die Maßnahme selbst für den Einzelnen, sondern auch die gesamte Gesellschaft durch eine Veränderung der Gesprächskultur. 
Die Entscheidung des Bundesverfassungsgerichts wurde ergänzt durch die abweichenden Meinungen der Richterinnen JAEgER und HOHMANN-DenNHARDT, die nicht nur die Passagen der StPO kritisierten, sondern auch die Veränderung des Art. 13 GG als unvereinbar mit den Garantien des Artikel 79 (3) und der Menschenwürde ansahen:

»Denn zur Persönlichkeitsentfaltung bedarf es Rückzugsräume, in denen der Einzelne ohne Angst vor Überwachung sich selbst zum Ausdruck bringen und mit Vertrauten über persönliche Ansichten und Empfindungen kommunizieren kann. Gerade in einer Welt, in der es technisch möglich geworden ist, so gut wie jede Bewegung und Kommunikation einer Person zu verfolgen und aufzuzeichnen, dient die Privatwohnung dem Einzelnen mehr denn je als letztes Refugium, in dem sich die Freiheit seiner Gedanken unbeobachtet manifestieren kann. Sie ist damit als Ort Mittel zur Wahrung der Menschenwürde» (ebd.: 356).

Die Einschränkungen des Urteils konnten dazu beitragen, dass der Grad an Freiheit wieder tendenziell, aber nicht vollständig restauriert werden konnte. Wenn man dem Minderheitenvotum gefolgt wäre, hätte dies in weitaus größerem Maße realisiert werden können. Dies ist nicht geschehen, was die abweichenden Richter wie folgt kommentieren:

»Inzwischen scheint man sich an den Gedanken gewöhnt zu haben, dass mit den mittlerweile entwickelten technischen Möglichkeiten auch deren grenzenloser Einsatz hinzunehmen ist. Wenn aber selbst die persönliche Intimsphäre, manifestiert in den eigenen vier Wänden, kein Tabu mehr ist, vor dem das Sicherheitsbedürfnis Halt zu machen hat, stellt sich auch verfassungsrechtlich die Frage, ob das Menschenbild, das eine solche Vorgehensweise erzeugt, noch einer freiheitlich-rechtsstaatlichen Demokratie entspricht. Umso mehr ist Art. 79 Abs. 3 GG streng und unnachgiebig auszulegen, um heute nicht mehr den Anfängen, sondern einem bitteren Ende zu wehren« (ebd.: 373).

Dem ist an dieser Stelle nichts hinzuzufügen, es bleibt nur auf Kapitel 4 zu verweisen, das sich auch mit den erwähnten technischen Möglichkeiten beschäftigen wird. 


\subsection{Terrorismus II $-9 / 11$}

Der Terrorismus der RAF war um die Jahrtausendwende kaum mehr präsent. Der bekannte Rest der RAF war sicher weggesperrt und Nachfolgeorganisationen von ähnlicher Bedeutung hatten sich keine gefunden. Terrorismus erschien als ein innerstaatliches Problem wie etwa der Fall der Anschläge von Atlanta im Jahre 1996 oder den wiederkehrenden Attentaten der ETA in Spanien und der IRA in Nordirland. In der allgemeinen Wahrnehmung war Terror kein wichtiges Thema, sondern betraf in der Regel immer die Anderen und nicht das eigene Umfeld.

\subsubsection{Eine neue Form des Terrorismus}

Im Jahr 2001 wurde der Terrorismus wieder zum weltweit wichtigen Thema. Zwar gab es immer Terrorismus, aber selten trat er in solch spektakulärer und medienwirksamer Form auf. Am 11. September 2001 konnte man quasi live mitverfolgen wie zwei Passagierflugzeuge in die beiden Türme des World Trade Centers geflogen wurden, die im Anschluss daran in sich zusammenfielen. Zusätzlich wurde das amerikanische Pentagon angegriffen und ein weiteres Flugzeug stürzte $a b$, ohne ein Ziel zu treffen. Insgesamt starben durch diese durch das Terrornetzwerk ${ }^{1}$ Al-Kaida durchgeführten Anschläge rund 3000

1 Der Begriff Netzwerk soll hier schlichtweg für eine Organisation stehen, die weit verzweigt ist und diverse Untergruppen koordiniert handeln lassen kann. Eine leistungsfähige Logistik, das Fehlen »strenger Hierarchie» (HEINE 2004: 151) und ein ausreichender finanzieller Hintergrund ist eine wesentliche Bedingung für die Funktionsweise. Eine differenzierte organisationssoziologische Betrachtung findet sich bei MAYNTZ (2006: 251 ff.) 
Menschen (FolLATH/SPÖRL 2006: 77), was in der langen Liste von Anschlägen, die dieser Organisation zugerechnet wurden, einen absoluten Höchstwert bedeutet. Fast noch schlimmer und bedeutsamer als die Toten war der Symbolgehalt der Anschläge, der die westliche Welt in ihrem Inneren auf eigenem Boden traf. Er besagte: Ihr seid nicht sicher, der Terror kann Euch überall treffen. Das Kürzel 9/11 wurde zu einem Symbol für einen drastischen Umschwung, für eine Zäsur vor allem für die Geschichte der USA. Aus heutiger Sicht wird die Bedeutung dieser Anschläge als zentraler Wendepunkt nicht von allen geteilt. Während Autoren wie HIPPLER (vgl. 2004: 3) dies anzweifeln und die Anschläge in den weltpolitischen Kontext, der vom Verfall des OstWest-Konlikts mit einer Erstarkung Europas und anderer Akteure bestimmt ist, stellen, sprechen viele andere wie beispielsweise Hoffmann und Schoeller klar von einem Wendepunkt 11. Spetember (vgl. Hoffmann/SCHOELLER 2004). In der Folgezeit wurden diverse Attentate weltweit Al-Kaida zugerechnet, darunter die Anschläge von Bali 2002, Madrid 2004 und London 2005. Die Antwort der USA und ihrer "Coalition of the willing" erfolgte militärisch mit Angriffen auf Afghanistan und den Irak, die beide zu der Zerschlagung der bisherigen Regime, aber nicht zur Zerschlagung von Al-Kaida führten.

\subsubsection{Neue Sicherheitsbedürfnisse nach den Anschlägen}

Nach den Anschlägen erhöhte sich das Bedürfnis nach neuen Formen der Sicherheit auch in Deutschland. Die unmittelbare Konsequenz waren zwei Sicherheitspakete (I und II), die am 9. November und 14. Dezember in Rekordtempo verabschiedet wurden. Sie verstehen sich selbst als gesetzgeberische Antwort auf die Anschläge vom 11. September 2001 und gerade auch das 
Sicherheitspaket I (Gesetz zur Bekämpfung des internationalen Terrorismus oder Terrorismusbekämpfungsgesetz) nennt die Anschläge ausdrücklich als Anlass: „Mit den Anschlägen in den Vereinigten Staaten von Amerika vom 11. September 2001 hat die terroristische Bedrohung weltweit eine neue Dimension erreicht. Vorbereitung und Ausführung der Anschläge waren gekennzeichnet durch ein hohes Ausmaß an Brutalität, Menschenverachtung und Fanatismus. Hinter den Anschlägen steht ein staatenübergreifendes Netz logistischer Verknüpfungen und operativer Strukturen« (BTDS 14/7386: 35). Der Schutz vor dieser Bedrohung wird nun zur "gemeinsamen Aufgabe aller staatliche(r)« Kräfte (ebd.), was es erforderlich macht, dass sich die mit der Terrorismusbekämpfung unmittelbar betrauten Polizeibehörden eng mit anderen Sicherheitsbehörden zusammenarbeiten (ebd.). Gleichzeitig wird Risikominimierung und Früherkennung (ebd.) zum erklärten Ziel, das nur erreicht werden kann, wenn bereits existierende Kompetenzen um neue Befugnisse erweitert werden (ebd.). Das letztendliche Ziel ist es zu verhindern, dass potenzielle Täter gar nicht erst einreisen können oder möglichst frühzeitig als solche erkannt werden, was unter anderem durch die Intregration von biometrischen Merkmalen in Reisedokumente erreicht werden soll (ebd.: 36).

Die nach dem Vornamen des damaligen federführenden Innenminister Schily benannten »Ottokataloge« passierten in geradezu rekordverdächtiger Zeit den Bundestag (vgl. bpb.de 2008). Verändert wurden mit der Einführung des Sicherheitspaketes I der $\S 129$ b StGB. Nun kann die Mitgliedschaft und Unterstützung terroristischer Gruppierungen die außerhalb Deutschlands beheimatet sind, geahndet werden. Zugleich wurde das Vereinsgesetz erweitert und findet nun Anwendung auch auf fundamental-islamistische Religionsvereine. Das Sicherheitspaket II beinhaltete insgesamt 14 Gesetzesände- 
rungen, die u. a. in folgenden Maßnahmen resultierten: "Verbesserung der Möglichkeiten der finanziellen Rasterfahndung, (...) Sperrung der Guthaben von Mitgliedern terroristischer Vereinigungen und Organisationen (.), sowie erweiterte Datenzugriffsbefugnisse der Sicherheitsbehörden, vor allem auf Daten im Bereich des Bankenwesens und der Telekommunikation« (ebd.).

Gleichzeitig wurden eine ganze Reihe von Einrichtungen wie die Geheimdienste - Militärischer Abschirmdienst (MAD), Bundesamt für Verfassungsschutz (BfV), Bundesnachrichtendienst (BND) und Bundeskriminalamt (BKA) mit erweiterten Kompetenzen ausgestattet, das Vereinsrecht, Ausländer- und Asylverfahrensrecht und das Pass- und Personalausweisgesetz (biometrische Merkmal werden verschlüsselt speicherbar) wurden zudem angepasst. Das Bundesamt für Verfassungschutz erhält die wichtige Aufgabe der »Vorfeldaufklärung» (ebd.: 36), es soll »Bestrebungen (.) beobachten, die gegen den Gedanken der Völkerverständigung oder das friedliche Zusammenleben der Völker richten, da sie ein gefährlicher Nährboden für wachsenden Terrorismus sind« (ebd.). Im Mittelpunkt der nachrichtendienstlichen Beobachtung liegt dabei die Kontrolle von nationalen und auch internationalen Kontenbewegungen und Geldströmen. Dazu benötigte Zugriffsmöglichkeiten auf die Daten von Finanzdienstleistern sind ebenfalls ein Bestandteil des Gesetzesentwurfs.

Der Einsatz des IMSI-Catchers, der zur Ortung von Mobilfunktelefonen eingesetzt wird, fand die rechtliche Grundlage in §100 i StPO. Das BfV erhält Zugriff auf Daten von Banken, Luftfahrtdienstleistern und Postdienstleistern. An Bord von deutschen Flugzeugen können Beamte der Bundespolizei (damals BGS) als Skymarshals nach amerikanischem Vorbild mitfliegen.

Die Sicherheitspakete erschienen vor dem Hintergrund des amerikanischen Konzepts der »Total Information Awareness« (STRÖM 2003: 25), das 
2006 allerdings wieder aufgegeben wurde, als vergleichsweise zahmer Maßnahmenkatalog, der trotzdem kritisch kommentiert wurde. LEUTHEUSSERSCHNARREN-BERGERS Kritik soll hier referiert werden, da sie das breite Spektrum der Maßnahmen umfassend wiedergibt (vgl. LEUTHEUSSER-SCHNARRENBERGER 2005: 281f.). Sie stellt dabei Parallelen zu den USA fest und eine grundlegende Veränderung der Sicherheitsarchitektur zugunsten eines »Präventionsstaates« (ebd. 281). »Massive Misstrauens- und Überwachungsmaßnahmen« würden dabei durchgeführt, um Sicherheitsrisiken zu reduzieren. Ein Tatverdacht sei keine zwingende Voraussetzung mehr für entsprechende Ermittlungen. Zwei zentrale Aspekte werden weiterhin bemängelt: Zum einen werde die Tätigkeit von Polizei und Geheimdiensten verwischt und gleichzeitig immer mehr ins Vorfeld von Straftaten verlegt, was zusammen mit der ausufernden Rasterfahndung mit dem Rechtsstaatsprinzip nicht vereinbar sei. Zum anderen veränderten die Schilygesetze das Verhältnis von Bürgern zu ihrem Staat grundlegend: Die Bürger fänden sich in der Rolle des Dauerverdächtigen wieder und liefen Gefahr, ständiger Kontrolle und präventiver Überwachung ausgesetzt zu sein. Für den einzelnen Bürger lauere zudem die Gefahr, von der deutschen Luftwaffe nach dem 2004 verabschiedeten Luftsicherheitsgesetz abgeschossen zu werden, wenn man sich an Bord eines entführten Flugzeuges befände. Die notwendige Kontrolle der Kontrolleure würde aus Kostengründen abgelehnt.

\subsubsection{Bewertung}

Den kritischen Anmerkungen Leutheusser-SCHnARREnbergers kann man folgen, wenn man die Veränderungen des Freiheitsrahmens in Deutschland 
bewerten möchte. Gerade die unmittelbare Lebensbedrohung durch das Luftsicherheitsgesetz beeinträchtigt die Freiheit unmittelbar, vor allem, wenn in nervösen Zeiten bei entsprechenden Pannen (Funkausfall) ein einschlägiger Vorfall leicht denkbar ist. Neuen Techniken wie die Erfassung biometrischer Daten im Personalausweis gehen in ähnlicher Art und Weise mit einem enormen Risikopotenzial für Freiheit einher, wenn sie kombiniert mit entsprechender Kameratechnologie (vgl. BAUM 2007, zitiert nach SIETMANN 2007) zu informationsreichen Bewegungsprofilen beitragen.

All das und die generelle Verlagerung der Ermittlungstätigkeit ins Vorfeld von Verbrechen reduziert das Maß an Freiheit in der Gesellschaft. Die Eigenkontrolle sinkt, Fremdkontrolle wird zum dauerhaften Zustand. Das Erfüllen bestimmter Merkmale genügt, um langfristig immer wieder in das Visier von Fahndern zu kommen. Das Argument, wer nichts zu verbergen hat, habe auch nichts zu befürchten, erweist sich als Totschlagargument, das mit dem Verweis auf geradezu übermächtige Sachzwänge jegliche inhaltliche Auseinandersetzung (vgl. SCHÜLER-SPRINGORUM 1997: 44) unterbindet. Es ist dabei fraglich, ob die getroffenen Maßnahmen das nach den Terroranschlägen reduzierte Ausmaß an Sicherheit wiederherstellen können. Die jüngsten Anschläge verschiedener Terrorgruppen hatten sich ja auch nicht im polizei- oder geheimdienstfreien Raum abgespielt. In jedem Fall wird wiederum deutlich, dass der einzelne Bürger bei der Rückgewinnung seiner Freiheit auf Hilfe und Mitwirkung von übergeordneten Einrichtungen angewiesen ist. In diesem Fall war es wiederum das Bundesverfassungsgericht, welches das Luftsicherheitsgesetz recht rüde für nichtig erklärte (BvR 357/05), indem es vergleichsweise kurz angebunden auf nur 156 Seiten, auf Art. 35 GG, die Menschenwürde und das Recht auf Leben verwies. Man kann sich des Eindrucks nicht erwehren, 
dass das Bundesverfassungsgericht damit als einziges »Bollwerk« (BAUM, zitiert nach SIETMANN 2007) verbleibt, das in schöner Regelmäßigkeit Grundrechts-und damit auch Freiheitseinschränkungen wieder rückgängig macht.

\subsection{BKA-Gesetz von 2008}

Das BKA-Gesetz, das im Jahre 2008 auf den Weg gebracht wurde, steht in der fast schon gewohnten Tradition der vorangegangenen Sicherheitspakete und stellt einen weiteren Schritt hin zur Konzentration von Kompetenzen zugunsten des BKAs dar. Man hat das Gefühl, dass unabhängig von der politischen Situation und Couleur des jeweiligen Innenministers eine BKA-Gesetzesinitiative gestartet wird. Dies deutet in letzter Konsequenz darauf hin, dass der jeweils für die Sicherheit verantwortliche Minister sich den Sachzwängen nicht entziehen kann oder will und daher versucht, möglichst viele Kompetenzen und Befugnisse für die Behörden seines Ressorts zu erhalten. Kommt es zu dramatischen Veränderungen der Sicherheitslage wie zum Beispiel durch einen Terroranschlag, so taugen diese Initiativen auch zur Entschuldigung und Rechtfertigung: Man habe ja auf die Gefahrenlage hingewiesen und Kompetenzen gefordert, die Verantwortung läge deshalb bei denjenigen, die sich der Kompetenzausweitung entgegen gestellt hätten.

Von hier ist es kein großer gedanklicher Schritt hin zur Annahme, dass solche Ereignisse auch instrumentalisiert werden können, um politische Veränderungen zu ermöglichen. Ob dies aus politischer Verantwortung, aus Gründen der Wahltaktik oder auch aus egoistischen Machtmotiven heraus geschieht, lässt sich oftmals nicht klar einschätzen. Das BKA-Gesetz in der aktuellen Vorlage geht auf diverse Ideen und Konzepte des Innenministers 
Schäuble zurück, die in Anlehnung an seinen Vorgänger Schily auch gerne als Schäuble-Katalog bezeichnet werden. In verschiedenen Legislaturperioden wurden immer wieder entsprechende Vorstöße gestartet, die in der Regel nicht auf breiten Konsens trafen, da oftmals die Koalitionskonstellationen ungünstig für eine Umsetzung waren. KREMPL und KURI fassen die bekanntesten Vorschläge zusammen (KREMPL/KURI 2008):

- Nutzung der Maut-Daten, um schwere Verbrechen wie Mord, Totschlag und Terrorismus verfolgen zu können;

- Online-Durchsuchungen mit Hilfe des Bundestrojaners, um Anschlagpläne und Ähnliches ermitteln zu können. Alternativen zum Trojaner sind fest verbaute Hintertüren, so genannte Backdoors;

- Biometrische Daten sollen nicht nur im Pass hinterlegt werden, sondern auch bei den Meldeämtern;

- Ausbau der Anti-Terror-Datei, in der entsprechende Verdächtige und ihr extremistisches Umfeld registriert sind.

Das BKA-Gesetz, wie es der Bundestag gegen Ende des Jahres 2008 verabschiedete griff verschiedene Aspekte des Schäuble-Katalogs auf und nennt die wesentlichen Schwerpunkte:

»Neben den polizeilichen Standardbefugnissen werden dem BKA besondere Mittel der Datenerhebung sowie die Möglichkeit der Ausschreibung zur polizeilichen Beobachtung und der Rasterfahndung zur Verfügung gestellt. Insbesondere erhält das BKA die Befugnis zum verdeckten Eingriff in informationstechnische Systeme (sog. Onlinedurchsuchung). Auch erhält das BKA durch den Entwurf Befugnisse zur Überwachung der Telekommunikation, zur Erhebung von Verkehrs- und Nutzungsdaten sowie zum Einsatz von technischen Mitteln zur Identifizierung und Lokalisation von Mobilfunkendgeräten, die auch bereits in etlichen Polizeigesetzen der Länder vorgesehen sind. Ebenfalls enthalten ist eine Befugnis zur Wohnraumüberwachung. Der Entwurf beachtet dabei die Recht- 
sprechung des Bundesverfassungsgerichts zum Kernbereich der privaten Lebensgestaltung und zu den Fragen der Kennzeichnung, Verwendung und Löschung personenbezogener Daten sowie der Benachrichtigung« (BTD 16/10121).

Als zugrunde liegendes Problem und Ziel des Gesetzes benennt der Gesetzentwurf »die Verbesserung der Möglichkeiten bei der Bekämpfung des internationalen Terrorismus durch das Bundeskriminalamt (vgl. BTDS 16/10121: 1)«. Als Lösung bekommt das BKA in bestimmten Fällen die Aufgabe der »Abwehr von Gefahren des internationalen Terrorismus« (ebd.) mit »entsprechden Befugnissen« (ebd.) übertragen. Die gesetzlichen Regelungen, so die Gesetzesvorlage weiter, können nicht ganz allgemein zur Gefahrenabwehr angewandt werden, sondern lediglich »zur Verhütung von terroristischen Straftaten nach Artikel I $§ 4$ a Abs. 1 Satz 2 des Entwurfs und zur Abwehr von Gefahren für die öffentliche Sicherheit« (ebd.: 16). Mit Terrorismus meint der Gesetzentwurf explizit »islamischen Terrorismus«, wo die meisten Fälle zu erwarten seien (ebd.: 17), obwohl die tatsächliche Gefährdungslage im Vergleich mit Staaten wie den USA, Großbritannien oder Israel als relativ "geringer« (ebd.) angesehen wird. Gleichwohl sei grundsätzlich »jederzeit und überall« mit Anschlägen auf deutsche Einrichtungen im Inland und Ausland möglich.

Das Gesetz scheiterte zunächst im Bundesrat an den Einwänden einiger Bundesländer, fand aber durch die Tätigkeit des Vermittlungsauschusses - im Grunde wurden Geistliche und Strafverteidiger vor den Maßnahmen geschützt -, dann letztlich doch die Zustimmung (Bundesratdrucksache 860/08, 28. 11. 2008). Zusätzlich wurde der Richtervorbehalt auch bei Eilverfahren installiert, der dann entscheiden muss, welche Daten wegen ihres höchst privaten Charakters nicht verwendet werden dürfen (vgl. sueddeutsche.de 2008 b). 
Dem Gesetz schlug von mehreren Seiten Kritik entgegen. Zum einen wurde im Vorfeld versucht, das Gesetz vor allem wegen der Online-Druchsuchungen zu verhinderen - dies geschah unter anderem durch den Arbeitskreis Vorratsdatenspeicherung, der eine Onlinepetition einreichte (vgl. bka-petition.de 2008). Begehrlichkeiten weckte die Befugniserweiterung für das BKA auch beim Präsidenten des Bundesamts für Verfassungsschutz Fromm, der die Befugnis für heimliche Online-Durchsuchungen auch für seinen Geheimdienst einforderte (vgl. KREMPL 2008). Prantl kommentierte das Gesetz in der SZ auf gewohnt kritische Weise und bemängelt die folgenden Punkte (PRANTL 2008a):

- keine verbesserte Abgrenzung zwischen BKA und den LKAs;

- es gibt keine klar definierte Situation, in der Online-Durchsuchung unmöglich ist;

- der Kernbereich privater Lebensführung bleibt sehr antastbar;

- unklare Begrifflichkeiten und Hürden für verschiedene Grundrechtseingriffe;

- Anwälte, Ärzte und Journalisten sind nicht geschützt;

- keine Definition von internationalem Terrorismus.

Der Kritik Prantls kann man sich im Großen und Ganzen anschließen. Die fast schon obligatorische erneute Prüfung durch das Bundesverfassungsgericht steht noch aus, daher fällt zunächst das gewohnte Auf und Ab von gesetzlicher Maßnahme und anschließender Korrektur aus. Was bleibt, ist nur die Freiheitseinschränkung für den Bürger, der jederzeit befürchten muss, nach bestimmten Kriterien Opfer beispielsweise einer Online-Durchsuchung zu werden. Schon allein diese Maßnahme birgt ein enormes Risiko für den Einzelnen, die Kontrolle über einen privaten Kernbereich völlig zu verlieren. 
Computer sind heute keine simplen Rechner, sondern auch Kommunikationsmittel, über die fleißig gechattet, Emails versandt und eingekauft wird. Kriminelle werden sich effektiv gegen das Eindringen von Außen wehren können, eventuell ganz banal dadurch, dass sie mit entsprechendem Know-how gegen Online-Durchsuchungen vorgehen werden, etwa durch das Benutzen von WLAN-Hotspots oder fremden Funknetzen, in die man sich mit Freewaremitteln einfach einklinken kann. Der unbescholtene Bürger, der zudem nichts von den Maßnahmen gegen ihn weiß, wird sich kaum prophylaktisch schützen und verliert damit sein Persönlichkeitsrecht auf Privatheit als zentrales Element der Freiheitsrechte. Was bleibt, ist ein Absinken des Vertrauens in IT-Systeme und die Bedrohung der Privatsphäre durch technische Weiterentwicklung: Moderne Computer besitzen häufig eine eingebaute Kamera, die den Einblick in die eigenen vier Wände ermöglicht, ein Mikrofon ist ja schon tradtionell mit verbaut. Ein solches Szenario ist keine Zukunftsmusik, sondern Bestandteil moderner Antidiebstahlsoftware (gadgettrak.com 2008) und der Onlinezugriff ermöglicht langfristig den ultimativen Lausch- und Spähangriff.

Das BKA-Gesetz ist zudem der Versuch, den Vorgaben des Bundesverfassungsgerichts gerecht zu werden, die im Vorfeld nach einer Klage gegen das Verfassungsschutzgesetz für Nordrhein-Westfalen (Online-Durchsuchung) bereits eine entsprechende Entscheidung gefällt hatte. Dabei führte das Gericht das Grundrecht auf Gewährleistung der Vertraulichkeit und Integrität informationstechnischer Systeme (vgl. Leitsätze, BvR 370/07) ein, das besagt, dass »die heimliche Infiltration eines informationstechnischen Systems, mittels derer die Nutzung des Systems überwacht und seine Speichermedien ausgelesen werden können« (ebd.) nur dann zulässig sei, wenn ein »überragend wichtiges Rechtsgut« (ebd.) einer konkreten Gefahr ausgesetzt ist. Der 
ganze Vorgang sei nur unter Richtervorbehalt verfassungsgemäß, zudem müssten gesetzliche Regelungen getroffen werden, um den Kernbereich privater Lebensgestaltung zu schützen. In der Gesamtschau kann kaum ein Zweifel daran bestehen, dass das BKA-Gesetz den bislang bestehenden Freiheitsraum des Bürgers einschränkt. Ob eine Erhöhung der Sicherheit damit de facto einhergeht, ist nach momentanem Kenntnisstand nur sehr schwer einzuschätzen. Dies wird auch künftig nur sehr schwer möglich sein, weil aus ermittlungstaktischen Gründen nicht unbedingt zu erwarten ist, dass Fahndungserfolge, welche durch die gesetzlichen Neuregelung möglich geworden sind, detailreich öffentlich diskutiert werden.

\subsection{Zusammenfassung}

Die bislang beschriebenen Meilensteine der Entwicklung von Sicherheit und Freiheit machen deutlich, dass nicht jedes Ereignis eindeutig bewertet werden kann. Nach Rückgängen und Einschnitten entstehen oftmals Kompensationen, die bestimmte Veränderungen wieder revidieren. Ebenfalls problematisch ist das Einschätzen von Effekten, die erst im Zusammenspiel mit anderen Faktoren und mittel- und langfristig ihre Wirkung entfalten und die zunächst nicht offen zu Tage treten. Die folgende Abbildung (siehe Abbildung 14) zeigt nochmals in einer Übersicht die besprochenen Entwicklungen im zeitlichen Zusammenhang. Sicherheit und Freiheit wurden 1949 auf eine durchaus solide Basis gestellt, obwohl im Bereich der Sicherheit offensichtlich noch Handlungsbedarf gesehen wurde. Mit der Notstandsgesetzgebung wurden Voraussetzungen geschaffen, die Freiheit im Falle einer Krise durch einen Angriff von außen einschränken zu können. Die Befürchtungen der damaligen Kritiker, dass diese 


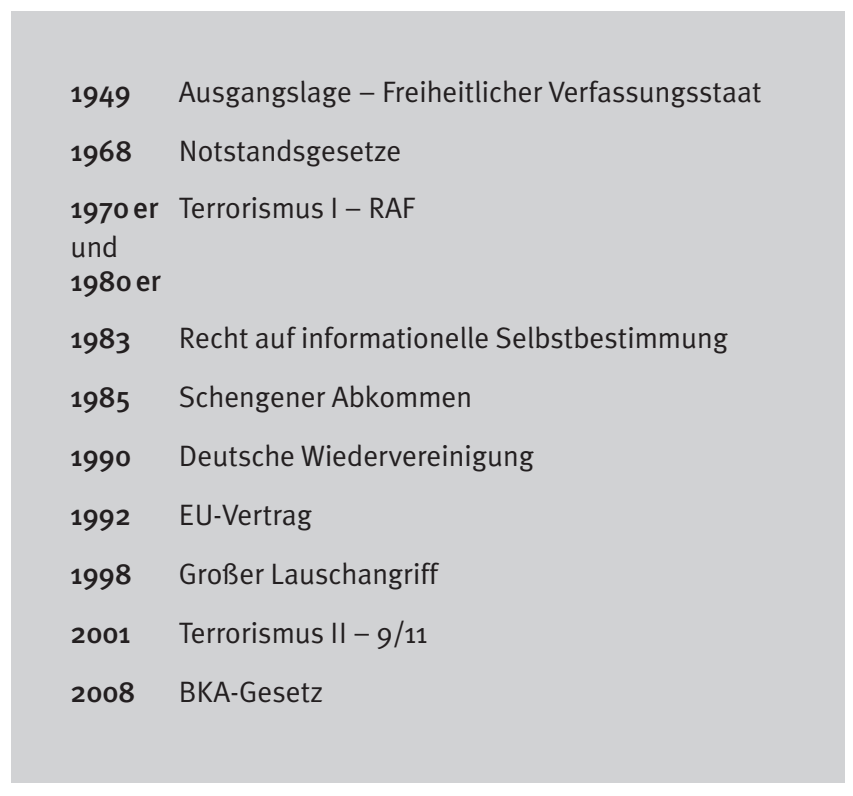

Abbildung 14 Chronologie der Entwicklung von Freiheit und Sicherheit

Gesetze missbraucht werden und die Freiheitsrechte unbegründet und unzulässig eingeschränkt werden könnten, haben sich bislang nicht bewahrheitet. Gestützt durch eine lange Phase der Entspannung, relativen Friedens und der europäischen Integration - in die EU, aber auch in die NATO -, musste noch nie über eine konkrete Anwendung nachgedacht werden.

Die erste Phase des Terrorismus wandte den Blick von der äußeren Bedrohung nach innen. Die Reaktion des Staates war, die Freiheit einzuschränken und so mehr Sicherheit zu ermöglichen. Der Terrorismus der RAF konnte zwar letztlich überwunden werden, allerdings muss man davon ausgehen, dass dies nur bedingt den Freiheitseinschränkungen geschuldet war. Trotz der Verhaftung "ganzer Generationen« von RAF-Mitgliedern erhob sich die RAF zumindest kurzfristig immer wieder als Phönix aus der Asche, der erst endgültig 
zu Grabe getragen werden konnte, als den Terroristen klar wurde, dass sich langfristig kein breiter Rückhalt in der Bevölkerung einstellte.

Das Recht auf informationelle Selbstbestimmung stellte einen Schritt hin zu mehr Freiheit dar. Das neu artikulierte Grundrecht beeinflusste maßgeblich die nachfolgende Rechtsprechung und das Datenschutzrecht. Das Bundesverfassungsgericht wurde zum ersten Male zu einem wichtigen Hüter der Freiheit. Die europäischen Einigungsprozesse waren über Jahrzehnte hinweg der wichtigste Garant für Freiheit in Europa. Die Lage Europas zwischen den zwei verfeindeten politischen Lagern des Kalten Krieges wirkte für diese Intergrationsprozesse als besondere Motivation.

Obwohl die politische Union nur in kleinen Schritten vorwärts kam, sorgte die wirtschaftliche Kooperation und die Schaffung des Binnenmarktes für eine stabile politische Umgebung. Als dann durch den Wegfall der Grenzkontrollen innerhalb des Schengenraumes, durch die gemeinsame Währung und der Einführung der vier Freiheiten (Freiheit für Waren, Kapital, Dienstleistungen und Personen) ein weiterer Schritt zu größeren Freiheiten gemacht wurde, erwachte seitens des Staates das Bedürfnis nach innerer Sicherheit in Gestalt von Grenzkorridoren, in denen die Schleierfahndung eingeführt wurde, die oftmals ohne konkreten Anlass ihr »Unwesen« treibt.

Der große Lauschangriff war ein herber Rückschlag gegen die Freiheit. Von der Unverletzlichkeit der Wohnung ist nicht viel geblieben, die Vertraulichkeit ist dahin. Man muss im Grunde darauf vertrauen, dass die Lauscher ausschalten, wenn es privat wird und dass so der Kernbereich privater Lebensführung auch privat bleibt.

Der Terrorismus von 9/11 machte aus einer simplen Folge von Zeichen und Ziffern, ein weltweit wiedererkennbares Symbol, das mit einer ganze Reihe 
unterschiedlicher Entwicklungen verbunden ist. Zum einen steht 9/11 für die Terroranschläge vom 11. September, bei der viele Menschen starben, zum anderen steht es auch für die Folgen: Krieg in Afghanistan und im Irak, die beide noch nicht beendet sind, das völkerrechtswidrige Lager von Guantánamo Bay und eine Welle von Sicherheitsgesetzen weltweit. Die Bedrohung der Freiheit und der Sicherheit durch Angriffe von außen führte zu einer Einschränkung der Freiheit im Inneren und einer massiven Ausweitung der Bemühungen um Sicherheit. Den letzten Endpunkt dieser Entwicklung markiert die OnlineDurchsuchung, die im BKA-Gesetz von 2008 verankert wurde. Ähnlich wie beim Großen Lauschangriff sorgte das Bundesverfassungsgericht für Korrekturen, so dass Hürden für den Eingriff in die Freiheit entstanden. Gleichwohl wurde die Chance verpasst, einen Grundrechtseingriff klar und vollständig zu unterbinden. 


\section{Aktuelle Entwicklungen - Das Verhältnis von Sicherheit und Freiheit}

Die verschiedenen wichtigsten Entwicklungen, die bislang vorgestellt wurden, sind Orientierungspunkte in einem langfristigen Prozess, der keinesfalls abgeschlossen ist. Verschiedentlich wurde die Freiheit eingeschränkt, dann wurden diese Einschränkungen wieder relativiert und ein Schritt wieder in die andere Richtung getan. In der Gesamtschau ging der Trend dahin, dass die Reduktion von Freiheit im Zuge des Strebens nach einer Erhöhung der Sicherheit billigend in Kauf genommen wurde. Die nun folgenden Ausführungen versuchen, den Entwicklungen und auch den wichtigsten Akteuren nachzugehen, die zur heutigen Situation geführt haben und die auch künftige Entwicklungen maßgeblich prägen werden.

\subsection{Der Wandel von Privatheit}

Wie gezeigt werden konnte, ist Privatheit ein zentrales Wesensmerkmal von Freiheit, da nur in ihr die enorm wichtige Eigenkontrolle und Autonomie ihren Ausdruck und Realisierung findet. Besonders wichtig sind dabei die verschiedenen Kontrollgrade, die dem Einzelnen zur Verfügung stehen und die Mittel, mit denen er die Kontrolle aufrecht erhalten oder sogar über das aktuelle Maß hinaus vergrößern kann. Die aktive Rolle des Einzelnen ist an dieser Stelle von entscheidender Bedeutung, da die subjektive Sichtweise und die Wahrnehmung der Situation das soziale Handeln und das Problembewusstsein definieren. 


\subsubsection{Schnittstellen und Kernbereiche}

Privatheit lässt sich nach Sphären ordnen, die vom Körper des Individuums ausgehend den Distanzbereich strukturieren. Die einzelnen Sphären sind keine hermetisch abgeschlossenen Räume, sondern sie besitzen Schnittstellen in Richtung Öffentlichkeit über die häufig kommuniziert wird. Privatheit im Sinne von Freiheit bezieht sich auf die Kontrolle durch das Individuum selbst, die über diese Schnittstellen ausgeübt wird. Im Kern der Sphären steht die Physis, also die körperliche Integrität des Einzelnen. Eingriffe in diesen Bereich respektieren nur dann unsere Freiheit, wenn wir sie aktiv erlauben, etwa dadurch, dass man sich beim Arzt eine Spritze geben lässt oder indem wir sie zeitweilig und nach bestimmten Bedingungen, wie zum Beispiel bei der Teilnahme an einer Kontaktsportart, zulassen. Die eigenen vier Wände ergänzen den körperlichen Bereich und erweitern ihn räumlich: Sie sind quasi die äußere Haut, der zweite Schutzgürtel des Individuums. Darüber hinaus erweitern sich die Sphären erheblich und die Kontrolle sinkt, wenn von außen z. B. durch so genannte Sicherheitsmaßnahmen staatlicher Organe ohne Zustimmung der Betroffenen eingegriffen wird. Als Kommunikationsschnittstellen sind hier Telefon, TV, Post und der Computer wichtig. Jenseits dieser Schnittstellen wird der Nahbereich der Privatsphäre verlassen und es bleibt nur noch die Anonymität als Teil der Privatheit.

Eine wichtige Schnittstelle, die vor allem in Bezug auf künftige technische Entwicklungen von erheblicher Bedeutung ist, ist der Computer. In Verbindung mit der Anbindung an Datenleitungen beinhaltet er das Potenzial, zum wichtigsten Kommunikationsmittel zu werden. Zum einen ist der jeweilige Benutzer damit in der Lage, direkt mit anderen Menschen zu kommunizieren, heute 
etwa über Chatsysteme, Emails und soziale Netzwerke. Mittlerweile ist es ebenfalls Standard, den Computer als Telefonersatz per Voice Over IP-Technik (DAVIDSON U.a. 2007: 25) zu benützen. Zum anderen hat der Benutzer damit Zugang zu einem praktisch unbegrenzten Informationsreservoir, das über die graphische Oberfläche des World Wide Web abrufbar ist und das auf Grund seiner dahinter verborgenen Netzstruktur ein hohes Maß an Stabilität aufweist. Eine weitere Möglichkeit, das Internet zu benützen, findet sich in Form von Peer-to-peer-Netzwerken, in denen mittels bestimmter Transferprotokolle wie TCP/IP (vgl. MAHLMANN/SCHINDELHAUER 2007: 7) praktisch jede Datei zwischen den Teilnehmern sich tauschen lässt. Wenn sich dann die Datei weiter im Netzwerk verbreitet, steigt die Geschwindigkeit der Verbreitung durch parallele Downloads von verschiedenen Peers drastisch an.

Die Fäden des gesamten Netzes sind die Links, welche die verschiedenen Inhalte im Netz verbinden und über die auf andere Informationen referenziert wird. Jede dieser Webseiten besitzt eine einmalige Adresse und kann über jeden Internetbrowser aufgerufen werden. Da ein Überblick über alle Adressen für den Einzelnen nicht möglich ist, übernehmen Suchmaschinen die Orientierung, indem sie kontinuierlich das gesamte World Wide Web automatisch indizieren und somit über bestimmte Stichworte mehr oder weniger gezieltes Suchen ermöglichen. Die Trefferquote ist dabei mittlerweile äußerst hoch, so dass im Prinzip mit den richtigen Suchbegriffen nahezu jede Webseite zu finden ist. Die Datenbank, welche der Tätigkeit der Suchmaschinen zu Grunde liegt, dürfte die mächtigste und bedeutsamste ihrer Art sein, da sie durch ihre Trefferangaben maßgeblich dazu beiträgt, welche Seiten besucht werden und welche Informantionen so zugänglich werden. Die Firma Google besitzt als Quasimonopolist in diesem Bereich den größten Einfluss. Im Bereich der Nach- 
schlagewerke besitzt die Online-Enzyklopedie Wikipedia die dominante Position bei der Bereitstellung von Wissen. Getragen von den Beiträgen der Benutzer ist auf diese Weise eine breite Informationsbasis entstanden, die jedem Menschen mit einem Internetzugang und einem Computer zur Verfügung steht.

Das letzte Beispiel des Wissens, dass das World Wide Web zur Verfügung stellt, zeigt, wie wichtig es für den Einzelnen ist, die Schnittstellen zu kontrollieren, welche die private Sphäre mit der Außenwelt verbinden. Nur wenn man sich der verschiedenen Schnittstellen bedienen kann, ohne Sanktionen befürchten zu müssen, ist davon auszugehen, dass der Betreffende sich einerseits frei artikulieren und andererseits mit Informationen und Wissen versorgen kann. Die Problematik, dass die Informationen, die das World Wide Web zur Verfügung stellt, keineswegs alle objektiv wahr sind, ist in diesem Zusammenhang zunächst sekundär und ein nachrangiges Problem, obwohl bewusst sein muss, dass gerade den Suchmaschinen und Online-Portalen, die Inhalte vorselektieren, hierbei eine wichtige Rolle zukommt.

\subsubsection{Eingriffe in die Privatheit - Kontrollverluste}

Die privaten Sphären existieren aber nur als normatives Konstrukt und als Ideal. Faktisch werden sowohl der private Kernbereich der eigenen vier Wände und gleichzeitig mit den Kommunikationskanälen die Verbindungen zu anderen Menschen überwacht. Der Große Lauschangriff betrifft diesen Kernbereich und gleichzeitig auch engste Familienangehörige, Freunde oder Gäste des Überwachten, die zugegen sind. Die folgende Abbildung 15 dokumentiert die Anzahl der zwischen 1998 und 2003 durchgeführten Wohnraumüberwachungen. 


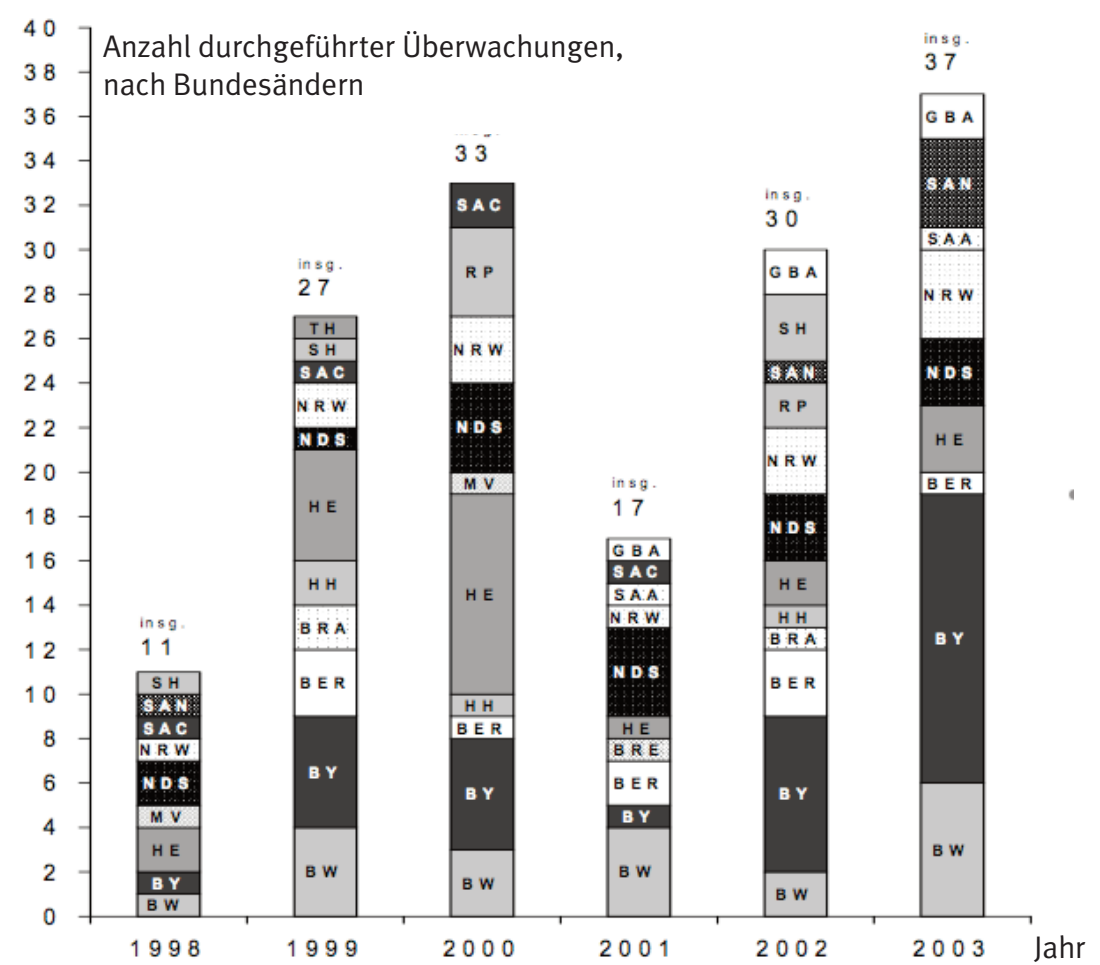

Abbildung 15 Langsschnittverteilung der nach Art. 13 Abs. 6 GG berichteten Verfahren mit Wohnraumüberwachungsmaßnahmen nach § 100 c Abs. 1 Nr. 3 StPO (abgeändert und ergänzt, vgl. MEYER-WIECK 2004: 24)

\begin{tabular}{|lll|lll|}
\hline Jahr & TKÜs & $\begin{array}{l}\text { Steigerungsrate } \\
\text { (in \%) }\end{array}$ & Jahr & TKÜs & $\begin{array}{l}\text { Steigerungsrate } \\
\text { (in \%) }\end{array}$ \\
\hline 1990 & 2494 & & 1997 & 7776 & +21 \\
1991 & 2797 & +12 & 1998 & 9802 & +26 \\
1992 & 3499 & +25 & 1999 & 12651 & +29 \\
1993 & 3964 & +13 & 2000 & 15741 & +24 \\
1994 & 3730 & -6 & 2001 & 19896 & +26 \\
1995 & 3667 & -2 & 2002 & 21874 & +10 \\
1996 & 6428 & +75 & Gesamt & & +777 \\
\hline
\end{tabular}

Abbildung 16 Steigerungsraten der Telekommunikationsüberwachungen im Vergleich zum jeweiligen Vorjahr (abgeändert, vgl. ALBRECHT u.a. 2003: 30) 
Praktisch jede der Kommunikationsschnittstellen kann heute ebenfalls durch staatliche Behörden überwacht werden. Neben den Lauschangriffen der Ermittler auf die Wohnungen wurden zum Beispiel parallel dazu eine Vielzahl von Telefonüberwachungen (siehe Abbildung 16) in Deutschland durchgeführt. Diese Daten dazu entstammen einer Studie des Max-Planck-Instiuts für ausländisches und internationales Strafrecht, die sich mit der »Rechtswirklichkeit und Effizienz der Telekommunikation nach den $\S \S 100 \mathrm{a}, 100 \mathrm{~b}$ StPO und anderer verdeckter Ermittlungsmaßnahmen« (ALBRECHT 2003) ausführlich beschäftigte. Die Studie versuchte, den Umfang der Telekommunikationsüberwachung (TKÜ) und die Rolle „von Polizei, Staatsanwaltschaft, gemessen an Anordnungshäufigkeit, Befristung der TKÜ-Maßnahme und tatsächlicher Überwachungsdauer« (ebd.: 431) zu erforschen und eine »Evaluation der Effizienz« durchzuführen. Zu diesem Zweck wurden 523 Verfahrensakten analysiert, rund 6000 Personen befragt, die als Praktiker mit der Materie befasst waren und in einem letzten Schritt 43 Experten (ebd.: 433 f.) interviewt.

Die Studie wurde von BIZER ausführlich kommentiert (vgl. BIZER 2003: 280 ff.) und die verschiedenen Ergebnisse und Erklärungsansätze kritisch beleuchtet. Den massiven Gesamtanstieg der TKÜ-Anordnungen um insgesamt 777 Prozent von 1990-2002 (siehe Abbildung 16) beispielsweise erklärten die Autoren der Studie mit drei verschiedenen Sachverhalten (AlBRECHT 2003: 30): 1. Mit einer Zunahme von bestimmten Kriminalitätsformen, bei denen «die Überwachung der Telekommunikation Ermittlungsprobleme überwinden hilft« (ebd.); 2. Als Reaktion auf die »dynamische Entwicklung« (ebd.) des Telekommunikationsmarktes und die damit einhergehenden veränderten Telekommunikationsgewohnheiten; 3. Die verdeckte Überwachung wird zunehmend zum Ermittlungsstandard. 
BIZER merkte dazu an, »dass die Verfügbarkeit elektronischer Überwachungstechniken im Arsenal polizeilicher Ermittlungsmethoden ihre Attraktivität ebenso beding(e) wie die zunehmende Nutzung des Mobiltelefons durch die Bürgerinnen und Bürger« (BIZER 2003: 284).

Die weitere Kritik BızERS an der Praxis der TKÜ - und auch an der Interpretation der Daten durch die Autoren der MPI-Studie - lässt sich in Form von sechs Aussagen zusammenfassen:

1. Nur 60 Prozent der TKÜ-Maßnahmen war erfolgreich, die Bedeutung als Beweismittel war gering und wurde nur bei 12 Prozent der Fälle realisiert (vgl. BIZER 2003: 282f).

2. Die TKÜ ist eine Maßnahme der Ermittlung und nicht der Beweisführung. Sie wird proaktiv »in Form von Vor- und Strukturermittlungen unter Einbeziehung von Erkenntnissen aus anderen Verfahren und im Rahmen von Initiativermittlungen« (ALBRECHT 2003: 155) verwendet (vgl. BIZER 2003: 283).

3. Die Aktenlage ist von schlechter Qualität und in mindestens der Hälfte der Fälle lückenhaft. Offen bleibt unter anderem, ob ein Zeugnisverweigerungsrecht bestand und ob die Maßnahme allen Betroffenen nachträglich mitgeteilt wurde (vgl. ebd.: 285).

4. Die Kontrolle der Maßnahmen durch den Richter ist de facto nicht gegeben. Lediglich 22 Prozent der Anträge auf eine TKÜ durch die Staatsanwaltschaft ging über bloße Formeln hinaus und war substanziell begründet (ebd.).

5. Es ist nicht zu ermitteln, ob die Kosten der Überwachungen dem Nutzen angemessen sind, da die Verwendung von finanziellen Ressourcen im Zusammenhang mit den Maßnahmen nicht entsprechend erhoben werden.

6. Es gibt keine systematische Erfassung aller TKÜ-Maßnahmen als valide Basis für eine gründliche Evaluierung (vgl. ebd.: 289). 
Den kritischen Anmerkungen Bizers kann man sich nur in vollem Umfang anschließen. Dass in vielen Fällen von Telekommunikationsüberwachung der Richtervorbehalt nicht im beabsichtigten Sinne funktioniert, nämlich dann, wenn die Begründung der Maßnahme formelhaft geschieht und nicht präzise auf den Einzelfall eingeht (u.a. bei Kinzig 2004, Albrecht u.a. 2003 und DoRSCH 2005), ist wirklich bedenklich. Es stellt sich die Frage, ob die Richter die nötigen finanziellen und zeitlichen Ressourcen besitzen, um die verschiedenen Fälle substanziell prüfen zu können. In jedem Fall scheint es viele Richter zu geben, welche die vorgelegten Anträge durchwinken. Eng damit in Zusammenhang steht die mangelhafte Benachrichtigung der Betroffenen. Genehmigen die zuständigen Richter auch deswegen die Überwachung, weil sie davon ausgehen, dass die Betroffenen sowieso nicht informiert werden? Die für die Freiheit so wichtige Eigenkontrolle ist durch die oben genannten Aspekte praktisch unmöglich. Zusammen mit der ungenauen Aktenführung ist es für den Einzelnen unmöglich, sich ein präzises Bild von den Überwachungsmaßnahmen zu machen, die ihn betreffen. Somit muss man von einer massiven Einschränkung der Freiheit ausgehen, die sich nur durch einen grundlegenden Wandel des Umgangs mit der Überwachung wieder herstellen lässt - im Falle eines offenen Umgangs und einer umfassenden Information der Betroffenen müssten die Überwacher aber damit rechnen, dass die Rechtmäßigkeit der Maßnahmen ausführlich erörtert und überprüft werden würde. Offensichtlich möchte dieses Risiko aber niemand eingehen.

Für ähnlich aufschlußreiche Zahlen und Untersuchungen bezüglich von Online-Durchsuchungen ist es noch zu früh, es ist aber auf Grund von Medienberichten davon auszugehen, dass zumindest im internen Netzwerk des BND diese Technik an Mitarbeitern ausprobiert wurde (vgl. MueHLbaUer 2008). 
Auch außerhalb des unmittelbaren Privatbereichs gehen die Eingriffe in die Privatheit weiter. Das oft gepriesene Bankgeheimnis existiert nicht mehr, verschiedene Behörden greifen heute vermehrt auf Konten zu. Für das Jahr 2006 wurden 93560 Fälle registriert, in denen Steuerfahndung und Strafverfolgung Zugriff auf Kontostammdaten hatten (vgl. KURI 2008). Die Kosten für die Infrastruktur, die bis zu 10000 Abfragen am Tag ermöglichen soll, tragen mit ziemlicher Sicherheit die Kunden (vgl. ebd.). Des Weiteren bedrohen Rasterfahndung (vgl. Kapitel 3), Gendateien, biometrische Gesichtserkennung und eine umfassende Videoüberwachung, die zunehmend auch durch mobile Überwachungsanlagen durchgeführt wird, das freie Bewegen in der Öffentlichkeit. Der Anspruch des Bürgers, in Ruhe gelassen zu werden und auf Eigenkontrolle über die Kommunikationsschnittstellen, geht zumindest tendenziell verloren.

\subsection{Akteure in der Überwachungsgesellschaft}

Als Konsequenz erscheint unser Staat oftmals als ein Überwachungsstaat, der alle Mittel einsetzt, seine Mitglieder zu überwachen. Es ist aber wesentlich exakter, von einer Überwachungsgesellschaft zu sprechen, da heute nicht mehr nur der Staat überwacht und in die Freiheiten eingreift. Der schottische Soziologe Lyon hat diese Überwachungsgesellschaft in einer Vielzahl von Veröffentlichungen näher untersucht und beschrieben. Für ihn ist die Überwachungsgesellschaft - im Original »surveillance society« - dadurch gekennzeichnet, dass ein Fülle an persönlichen Daten eines jeden Menschen, gesammelt, aufgezeichnet und verarbeitet wird (vgl. Lyon 1994: 3). Ein wichtiges Kennzeichen dieser Gesellschaft ist nach Lyon die Tatsache, dass diese Vorgän- 
ge keineswegs besonders außergewöhnlich seien, sondern dass sie sich gewissermaßen im gelebten Alltag etabliert haben: Was jahrzehntelang einst zum Repertoire von Spionen und Agenten gehörte, wie das Abhören und Belauschen (vgl. ebd.), kann nun jedermann betreffen. LYon weist daraufhin, dass Überwachung keinesfalls ein neues Phänomen sei (vgl. LYoN 2006: 3), sondern dass schon seit jeher Daten von den Mitgliedern einer Gesellschaft gesammelt worden seien - offensichtlich bestand ein grundsätzliches Interesse, bestimmte Informationen etwa Geburtstag, Einkommen oder Eigentum gezielt zu sammeln und auch zu speichern. Im Laufe der Zeit weitete sich dieses Interesse erheblich aus. REGAN erwähnt in diesem Zusammenhang die Überwachung am Arbeitsplatz, wo mit einsetzendem Kapitalismus der Bedarf nach Information und Kontrolle der Produktivität der Arbeitnehmer enstand (vgl. RegAn 1996: 21). Bis heute ist diese Kontrolle der Arbeitnehmer ein konflikthaltiges Thema, das immer wieder die gleichen Fragen aufwirft: Wieviel darf ein Arbeitgeber denn überhaupt kontrollieren und welche Mittel darf er dabei legal einsetzen?

Die heutige Überwachungsgesellschaft ist nach LYON durch zwei Phänomene charkaterisiert (vgl. LYON 1994: 4 ff.). Zum einen ist dies die Routine, mit der Überwachung zwar allgegenwärtig ist, aber trotzdem oftmals unbemerkt in dem Sinne abläuft, als Daten im Hintergrund gesammelt und verarbeitet werden. LYON beschreibt dies sehr anschaulich an seiner Übersiedlung nach Kanada, in dessen Verlauf er Führerscheine, Sozialversicherungsnummer und Krankenversicherung beantragen muss und jedes mal elektronisch in einer Datenbank registriert wird, wobei auf die so geschaffenen Datensätze dann im weiteren Verlauf bei diversen Anschaffungen, wie etwa einem Hauskauf, zugegriffen wird (vgl. ebd.). 
Neben der Routine der Erfassung, ist laut LYoN die computergestützte Verarbeitung der Daten charakteristisch für die Überwachungsgesellschaft. Über Computer und die verschiedenen Kommunikationskanäle werden die gesammelten Daten zusammengeführt und abgleichbar gemacht (vgl. ebd.: 4). Auf diese Weise hinterlässt jeder Bürger im ganz alläglichen Leben eine große Anzahl »elektronischer Spuren« (EBD.), die auf verschiedenen Medien gesammelt werden können. Als zentralen Ausgangspunkt für alle Formen der Überwachung nennt LYON die Identifikation des Einzelnen (vgl. LYON 2009: 4): Erst wenn die verschiedenen Datensätze bestimmten Personen eindeutig zuzuordnen sind, werden die Daten für die Überwacher erst brauchbar und sinnvoll, wobei die »ID-cards« als Mittel der Identifikation selbst verstärkt auch zum Träger von höchst sensiblen Informationen werden können. LYON nennt in diesem Kontext konkret das Beispiel Finnland, wo neben Daten, die über den Stauts als Bürger Auskunft geben, wie Alter, Größe oder Geschlecht, gleichzeitig Gesundheitsinformationen gespeichert werden (ebd.: 16), während man als deutscher Bürger heute an auf Reisepässen gespeicherte Fingerabdrücke und an die Erfassung biometrischer Daten denkt. Die Überwachungsgesellschaft insgesamt ist nach Lyon in ständigem Wandel, was die Überwachungsmethoden angeht, zudem intensiviert sich das Ausmaß an Überwachung insgesamt (vgl. LYON 1994: 38).

Das von ORWELL beschriebene Szenario eines alles erfassenden Big Brothers, der bis ins Intimste seiner Bürger blickt, ist in der Überwachungsgesellschaft abgelöst von vielen verschiedenen kleinen Überwachern. Statt monolithischer staatlicher Kontrolle (vgl. GARFINKEL 2001: 3) agieren nun hunderte von »Kid Brothers«, die alles und jeden dauerhaft beobachten. Es macht die Überwachungsgesellschaft aus, dass diese Überwachung absichtlich, routiniert, syste- 
matisch und konzentriert (vgl. BALL/WOOD 2006: 4) erfolgt. Überwachung wird zum Bestandteil des Alltags und sie funktioniert getreu dem Motto: „Wer nichts zu verbergen hat, hat nichts zu befürchten« (PRANTL 2002: 9).

\subsubsection{Behörden und staatliche Organe}

Behörden wie Polizei, Staatsanwaltschaft oder Finanzämter sind klassische Überwacher, die mit ihrer Tätigkeit ein klares Interesse verfolgen. Ziel ist das Aufspüren von Gesetzesverstößen und die Ermittlung von Tätern und Schuldigen. Handlungsweisen der Behörden sind an Recht und Gesetz gebunden. Aus Legitimitätsgründen haben sie ebenso wie die betroffenen Bürger ein berechtigtes Interesse an dieser rechtlichen Grundlage. Doch nicht alle staatlichen Einrichtungen und Behörden, sei es auf Bundes- oder Landesebene, sind Überwacher und Kontrolleure. Es finden sich neben den Gerichten, welche die Kontrolleure hinsichtlich Recht und Gesetz überwachen, auch Einrichtungen, die für die Freiheit und Privatheit des Einzelnen eintreten. Eine wichtige Rolle kommt in diesem Zusammenhang den Datenschutzbeauftragten zu. Basierend auf den Datenschutzgesetzen, die seit den 1970 und 1990 er Jahren verabschiedet wurden (vgl. Redelfs 2003: 205), nehmen sie die Rolle des mitunter einsamen - Rufers in der Wüste wahr. Besonders der Bundesdatenschutzbeauftragte Peter SCHAAR meldet sich regelmäßig in der politischen Diskussion zu Wort und fordert bei vielen emotional diskutierten Themen Versachlichung. Ein Blick auf die Webseite zeigt unter www.bfdi.bund.de die aktuell relevanten Themen: ELENA (Beantragung von Sozialleistungen durch ein elektronisches Verfahren, Vorratsdatenspeicherung, Passagierdatenübermittlung, Terrorismusbekämpfung, elektronische Gesundheitskarte, Biometrie (vgl. bfdi.bund.de 2008). Die wichtigste Tätigkeit des Beauftragten liegt in der 
Anfertigung regelmäßiger Datenschutzberichte, in denen das sensible Thema Datenschutz aufbereitet wird. Seine Behörde und entsprechende Pendants auf Landesebene stellen umfassende Informationen für die Bevölkerung bereit und nehmen in ihren Tätigkeitsberichten detailliert zu verschiedenen Sachverhalten Stellung. Neben Entschließungen und Empfehlungen sind diese Publikationen das einzige Mittel, öffentlichkeitswirksam Verstöße und Auffälligkeiten anprangern zu können - ansonsten bestehen keine Möglichkeiten, konkrete Sanktionen bei Verstößen zu ergreifen.

Mit dem Bundesamt für Sicherheit in der Informationstechnik (vgl. bsi.de 2008) gibt es eine weitere Bundesbehörde, die ähnliche Themen wie der Bundesdatenschutzbeauftragte aufgreift. Die Methodik des Amtes ist ebenfalls ähnlich. Es werden neue technologische Entwicklungen in sicherheitsrelevanten Bereichen besprochen, unter anderem auch die aktuellsten Trends und Sicherheitsmaßnahmen im Bereich von Schadsoftware (vgl. ebd.). Staatliche Bedrohung der individuellen Sicherheit wird nur indirekt zum Thema, etwa wenn das Amt beispielsweise auf einen Trend weg vom Computervirus und hin zum Trojaner feststellt (vgl. ebd.): Mit hoher Wahrscheinlichkeit funktioniert die im BKA-Gesetz verankerte Online-Durchsuchung auch mittels eines solchen Bundes-Trojaners.

Das Bundesverfassungsgericht ist eine weitere staatliche Einrichtung, die eine wichtige Rolle im Überwachungsstaat einnimmt, indem es seine speziellen Aufgaben innerhalb des gewaltenteiligen Systems der Bundesrepublik wahrnimmt. Mit den schon angesprochenen Urteilen und dem Artikulieren von neuen Grundrechten wie dem Recht auf informationelle Selbstbestimmung positionierte sich das Verfassungsgericht als wichtiger Gegenspieler, welcher der Errichtung eines Überwachungsstaates entgegen stand. 
Diese Einschätzung der Bedeutung des Bundesverfassungsgerichts ist prinzipiell richtig. Gerade aus der Möglichkeit, nichtverfassungskonforme Gesetze zu kassieren oder mit Auflagen zu versehen, die oftmals dem Gesetzgeber - vor allem auch aus dessen Sicht - fast schon zu genaue Vorgaben machen, wächst dem Gericht eine große Macht zu, die durch die Unabhängigkeit der Richter abgesichert wird. Gebunden ist das Gericht aber an die Kontinuität seiner eigenen Entscheidungen, an seine Verantwortung und an den Anspruch, die Verfassung ausgewogen und korrekt zu interpretieren. Viele Eingriffe in die Freiheit zugunsten von Sicherheitsbestrebungen werden deshalb vom Gericht zwar zurückgewiesen, aber im Gegenzug langfristig doch nur mit Hürden versehen: Das gilt für das Volkszählungsurteil, für die Urteile zum Großen Lauschangriff und auch für die Entscheidung über die OnlineDurchsuchung in der nordrhein-westfälischen Variante. Die gesetzlichen Regelungen werden dann angepasst und müssen sich danach in der Alltagspraxis bewähren, wobei sich aber oftmals Probleme ergeben, welche die Hürden wieder relativieren können. Hierzu gehört beispielsweise die Auslastung der Gerichte, die vermutlich zumindest teilweise zu den oben erwähnten pauschalen Ermächtigungen für Telefonüberwachungen führten.

Der Bundestag übt ebenfalls Kontrolle aus, wobei diese Funktion hauptsächlich von den Oppositionsparteien realisiert wird, während die Regierungsmehrheit auf Grund ihrer engen Verzahnung mit der Exekutive - symptomatisch dafür sind z.B. die parlamentarischen Staatssekretäre, die meist nur wenig öffentliche Aufmerksamkeit erfahren - eher zurückhaltend und informell agiert.

Trotz dieser Barrierefunktion können diese Einrichtungen den Überwachungsstaat aber nur bremsen und nicht umgestalten. Für dieses Vorhaben 
fehlt sowohl dem Bundesverfassungsgericht als auch der Opposition die nötige legislative Kompetenz, obwohl die Bedeutung des Gerichts - oftmals auch mit kritischem Unterton - innerhalb des politischen Systems sehr hoch eingeschätzt wird (vgl. u.a. UEBERSCHAER 1962: 152 ff. und SÄCKER 1995: 189ff.), mitunter ist sogar von einem »Richterstaat« oder einer »Aristokratie der Roben« (vgl. ebd.: 189) die Rede.

Auf der europäischen Ebene ist noch der Europäische Gerichtshof für Menschenrechte zu erwähnen, der in jüngster Vergangenheit mit spektakulären Urteilen gegen britische DNS-Datenbanken (vgl. KOYDL 2008) vorging und damit in Großbritannien eine Art Verfassungsgerichtsbarkeit durch die politische Hintertür einführte. Noch ist allerdings offen, ob und wie in Großbritannien auf die Entscheidung politisch reagiert wird.

Zwei Probleme stellen sich für die Behörden, die überwachen. Was macht man zum einen mit einem Geheimdienst, der einen Skandal verursacht, indem er eine befreundete Nation und eine Journalistin (vgl. LEYENDECKER 2008) ausspioniert und sich auch noch dabei erwischen lässt? Dieser Fall ist Wasser auf den Mühlen der Kritiker der Überwachung und es stellen sich zu Recht Fragen nach einer effektiven Kontrolle der Kontrolleure und auch danach, ob man dies parlamentarischen Kontrollauschüssen überlassen darf, die ihre Erkenntnisse auf Grund der gesetzlichen Vorgaben nicht öffentlich machen dürfen.

Zum anderen werden die Überwacher auch selbst Opfer, wie etwa im Fall des Hackerangriffs vom Sommer 2007 (vgl. HAUCK 2008 und SCHMIEDER/HAUCK 2007), als chinesische Hacker trojanische Pferde in Form von pdf-Dokomenten und infizierten Worddateien vor der elektronischen Stadt des Bundesinnenministeriums abstellten, die dann durch die Behörde bereit- 
willig in dieselbe gezogen wurden: «Man will die eigene Bevölkerung mit einem Trojaner ausspähen, schafft es aber nicht, sich selbst dagegen zu wehren« (KURZ 2008, zitiert nach SCHMIEDER/HAUCK 2008). CHRISTINE KURZ vom Chaos Computer Club verwies in Zusammenhang (vgl. ebd.) mit diesem Vorfall auch kritisch auf den neu geschaffenen Paragraphen 202 C StGB, der pauschal Computerprogramme unter Strafe stellt, deren Zweck es ist, Passwörter und Sicherungscodes zu umgehen. Damit macht sich auch jeder strafbar, der ohne jegliche kriminelle Absichten entsprechende Aktionen simuliert, um Gegenmaßnahmen zu entwickeln.

\subsubsection{Gruppierungen}

Auf der nächsthöheren Ebene gibt es ebenfalls Überwacher und Einrichtungen, die sich gegen die Überwachung wehren. Klassischer Weise finden sich letztere Gruppierungen im Dunstkreis von Datenschutzvereinen, links oder liberal orientierten Gruppen und Bürgerrechtlern unterschiedlicher Couleur. Auf internationaler Ebene machte in den vergangenen Jahren die Electronic Frontier Foundation auf sich aufmerksam. Diese Einrichtung versteht sich als »leading civil liberties group« (eff.org), welche die Rechte der Bürger in der digitalen Welt vertritt. Schwerpunkt der Organisation ist die Aufklärung bezüglich Themen wie »Free Speech, Innovation, Intellectual Property, Privacy, Tranparency« (ebd.), wobei mitunter einzelne Betroffene gezielt in Gerichtsverfahren bei der Einforderung ihrer Rechte unterstützt werden. In eine ähnliche Richtung gehen die Aktivitäten der verschiedenen Vereine, die in Deutschland und in anderen Ländern die Big Brother-Awards in Zusammenarbeit mit weiteren unabhängigen Organisationen als »Auszeichnung« für den 
Missbrauch von Information und Technik verleihen. Im Bereich der Weblogs (Blogs) und der traditionellen Medien finden sich in Deutschland zahllose Akteure (z. B. SZ, heise online u. a.), welche die verschiedenen Entwicklungen des Überwachungsstaates kritisch begleiten. Wichtig ist für alle diese Gruppen die Meinungsfreiheit, welche die Basis für die Verbreitung der Kritik ist. Das World Wide Web entwickelte sich in diesem Kontext im Laufe der Zeit immer mehr zu einem wichtigen Medium, da dort zum einen günstig in Schrift, Ton und Bild publiziert und zum anderen ein relativ großes, internationales Publikum erreicht werden kann.

Es gibt darüber hinaus viele Einrichtungen, die nicht für die Privatheit eintreten, sondern selbst Überwachen oder zumindest Daten bereitstellen, die zur Überwachung verwendet werden können. Ein beliebtes Beispiel für eine solche Gruppierung ist die Firma Google, welche die gleichnamige Suchmaschine betreibt. Von ihr angeboten werden heute eine Fülle (vgl. REISCHL 2008:96) von Webdiensten, die alle sehr datenintensiv sind, darunter einen online-Bilderdienst, einen Email-Service, ein globales Kartographieprogramm, das mit Hilfe von digitalen Bilddaten arbeitet, eine Online-Textverarbeitung, ein Videoportal, ein Nachrichtenportal und vieles mehr. Das Programm Google Analytics ist in der Lage, Zugriffe auf Webseiten, auf denen es integriert wurde, auszuwerten und umfangreiche Statistiken zu erstellen. Gleichzeitig bietet Google kostenlosen Festplattenplatz für die Einrichtung von Webseiten für Firmen und Privatanwendern, auf denen Werbung geschaltet werden kann. Erfasst werden zusätzlich die Suchmuster von mehreren Hunderttausend Internetsurfern, welche die Suchmaschine tagtäglich intensiv benutzen. Google registriert diese, gleicht die ungeheure Datenvielfalt intern ab und speichert diese zur Zeit 1,5 Jahre lang. Diesbezügliche Kritik durch EU-Datenschützer (vgl. WILKENS 
2008) wies die Firma bislang mit Verweis auf die international unterschiedlichen rechtlichen Anforderungen zurück. Aus dieser Mischung gibt sich in Kombination mit dem Internetbrowser Chrome ein wahres Datendesaster, da nach jedem Download der Software (vgl. REISCHEL 2008: 90) diese über einen Code eindeutig identifizierbar wird. Jegliche Anonymität im Internet ist damit völlig zerstört und man kann nur hoffen, dass auf diese »hochexplosive« Datenbank niemand mit einem missbräuchlichen Interesse Zugriff erhält.

Google ist zwar der größte Datensammler, aber beileibe nicht der Einzige. Besondere Aufmerksamkeit verdienen auch die Firmen, auf die der Staat mit seiner Vorratsdatenspeicherung abzielt. Diese Firmen fallen immer dann auf, wenn sie Daten verlieren, missbrauchen oder Opfer von Kriminellen werden und auf diese Weise dann die Kontrolle über die Daten verlieren. Dies gilt zur Zeit für die Kunden der Berliner Landesbank, von denen Bankdaten und Geheimnummern kursieren (vgl. PRANTL 2008 b). Betroffen sind gleichfalls Mitarbeiter der Telekom und Journalisten, die im Auftrag der Telekomführung (vgl. SCHÄFER 2008) ausgespäht wurden. Der Bundesverband der Verbraucherzentralen konnte ebenfalls Zugriff auf illegale Daten bekommen, indem er insgesamt sechs Millionen Datensätze problemlos auf dem Schwarzmarkt erwerben konnte (vgl. freenet.de 2008). Die Datensammelwut und das Speichern und Weitergeben von Daten ist ein florierendes Geschäft, mit dem weltweit hohe Geldsummen umgesetzt werden und letztlich auch die Freiheitsrechte der Bürger verletzt werden können.

\subsubsection{Individuen - die Veröffentlichung des Privaten}

Die Ebene des Individuums ist in zweierlei Hinsicht relevant. Zum einen sind Individuen logischerweise an den Entscheidungen von staatlichen Einrichtun- 
gen und Gruppierungen beteiligt. Sie wirken an politischen Entscheidungen, Klagen oder Überwachungsaktionen unmittelbar mit. Durch die verschiedenen Entscheidungen, die sie treffen, verschieben sich mitunter Freiheit und Sicherheit. Bekleidet der Betreffende ein politisches Amt, so prägt das Amt inn und vice versa. Im Fokus bei politischen Entscheidungen steht dabei natürlich besonders der Innenminister, dessen Ressort auf Staatsseite die gesetzlichen Grundlagen braucht, um die Überwachung legal ausüben zu können. Die Person des derzeitigen Innenministers Wolfgang SCHäUble ist dabei für Kontroversen allemal gut, zumal er sein Anliegen durchaus offensiv vertritt und auch Verfassungsrichtern wie dem eigentlich als konservativ einzuschätzenden HANS-JÜRGEN PAPIER zuweilen recht harsch bedeutet, sie seien nicht demokratisch legitimiert und alle grundrechtlich geschützten Bereiche würden irgendwo enden:

»Alle grundrechtlich geschützten Bereiche enden irgendwo. Wo diese Grenzen sind, wie man die gegensätzlichen Interessen abgrenzt, ist Sache des Gesetzgebers. Ich verstehe, dass manche Verfassungsrichter gern Ratschläge geben würden. Dazu sind sie aber nicht demokratisch legitimiert« (SCHÄUBLE, zitiert nach JUNGHOLt/MÜLLER 2008).

Diese Aussage SchÄUbles ist zwar formal korrekt, trotzdem steht der Innenminister in Sachen demokratischer Legitimierung dem Souverän auch kaum näher als ein Verfassungsrichter - beide sind nicht unmittelbar vom Volk gewählt. Die Sprache und der Stil der Auseinandersetzung mit der Thematik vollzieht sich aber oft auf ähnliche Art und Weise. Geradezu ritualisiert wirkt die häufige Aufeinanderfolge von politischen Entscheidungen, Urteilen, Skandalen, Presseerklärungen, Stellungnahmen und Interviews anhand derer die verschiedenen Positionen, oftmals nicht frei von Polemik, der Öffentlichkeit präsentiert werden. Nicht immer geht es dabei um die Sache an sich, oftmals 
spielen politiktaktische oder persönliche Motive eine wichtige Rolle, wodurch die Themen Sicherheit und Freiheit nicht mehr nur rein sachlich Gegenstände der politischen Diskussion sind.

Neben den Entscheidungsträgern gibt es auf der individuellen Ebene noch die große Gruppe der von der Überwachung Betroffenen, die ihrerseits durch ihren Umgang mit der Thematik Einfluss auf die Überwachungsgesellschaft haben. Eine wesentliche Verhaltensweise ist dabei das Ignorieren der Eingriffe in das Privatleben, das nur allzu oft mit einem trügerischen Anonymitätsgefühl verbunden ist. Dies offenbart sich auch im Umgang mit dem World Wide Web, das ebenso sorglos verwendet wird (vgl. LeYENDECKER 2007: 2). Die Einzelfallrecherche von SöHLER, der einem seiner Chefredakteure im Netz nachspürt, ergibt mit einfachen Mitteln Erstaunliches (SöHLER 2008). Bei der Websuche, die ohne kostenpflichtige Recherchemöglichkeiten Auskunft erteilt, werden eine Unmenge an Daten entdeckt, vor allem auch über so genannte soziale Netzwerke, in denen Benutzer oftmals jede Menge privater Daten freigeben, die beispielsweise künftige Arbeitgeber nicht lesen sollten. Insgesamt lässt sich ein breiter Trend feststellen, private Daten selbst aktiv zu veröffentlichen oder sie zumindest nicht angemessen zu schützen. Dies gilt für die schon erwähnten sozialen Netzwerke und ebenso für Fernsehsendungen. Anscheinend kann man mit einer Kamera und einem Mikrofon ausgerüstet zu fast jedem Thema Aussagen sammeln und sich nebenbei gleich noch die Personalien mitnotieren. Rund um die Uhr sehen wir Fernsehsendungen, in denen Menschen sich mit viel Engagement selbst veröffentlichen, so dass wir Menschen beim Kochen, Weinen, Verzweifeln, Kinder- und Hundeerziehen und über Politik diskutieren sehen können. Abseits der Medien, in der realen Welt setzt sich der Trend des dauerhaften Distanzverlustes fort und man kann sich 
den Trends nur durch aktiven Rückzug entziehen. HASSEMER, damals Vizepräsident des Bundesverfassungsgericht, fasst die Veränderungen prägnant in drei Aussagen zusammen (HASSEMER 2000: 17):

- Das »Dabeisein in fremden Stuben und Seelen« (ebd.) sei zu einer »alltäglichen Form des Genusses« (ebd.) geworden und daraus werde eine Art Recht auf Dabeisein abgeleitet. Medien, welche die Interaktion erlauben, verstärken diese Mechanismen.

- „Die alten Mauern um das Private stürzen nach und nach ein« (ebd.). Es muss immer damit gerechnet werden, dass fremde Augen und Ohren zum ständigen Begleiter werden. Die Verteidigung des Datenschutzes und der Privatheit, SO HASSEMER, sei »ermattet« (ebd.): „Warum bist Du für den Schutz Deiner persönlicher Daten, wenn es nicht gewisse Gründe dafür gibt, dass diese Daten das Licht der Öffentlichkeit scheuen?«

- Im letzten Punkt verweist HASSEMER auf eine Veränderung des Schamgefühls und stellt fest, dass »um seiner selbst willen« (ebd.) heute nichts mehr verborgen werden muss. Die Gründe hierfür lägen in einer Veränderung der »Triebkräfte und Ängste(.)« (ebd.).

Die Konsequenz aus diesem Ausliefern in die öffenliche Privatheit, die gleichzeitig auch privatisierte Öffentlichkeit ist, ist für den Verfassungsrichter und Rechtssoziologen HASSEMER die schlichte Einsicht, dass Passivität keine angemessene Haltung gegenüber dieser veröffentlichenden und veröffentlichten Gesellschaft ist, sondern dass Privatheit Aktivität benötigt:

«Wer eine Vorstellung von Privatheit als Teil des guten Lebens hat, muss aktiv werden. Er muss diese Vorstellung in Bewegung bringen und sie auf die neuen Verhältnisse übersetzen; sonst versteht er die Welt nicht mehr. Erst nach dieser Übersetzung gibt es die Chance, die neuen Verhältnisse und die Wonnen der Privatheit so miteinander zu verbinden, dass etwas Vernünftiges daraus wird« (HASSEMER 2000: 17). 


\subsubsection{Exkurs - Gated Communitys}

An dieser Stelle soll ein kurzer Exkurs über die so genannten Gated Communitys eingeschoben werden, da diese Form des Zusammenlebens einige besondere Merkmale bezüglich Privatheit, Exklusion und Kontrolle und zudem Parallelen zur Überwachungsgesellschaft aufweist. Ohne an dieser Stelle ausführlich auf Unterschiede von »Lifestyle«, »Prestige« oder »Security Zone Communities« (BLAKelY/SNYDER 1999: V) zu differenzieren, kann man wesentliche Merkmale von Gated Communitys wie folgt zusammenfassen. Grundsätzlich versteht man unter den Communitys private Wohnsiedlungen, die als Gemeinsamkeit besondere Sicherheitsmerkmale aufweisen: „Walls are only the beginning. Inside may be surveillance cameras, infrared sensors, motion detectors and sometimes armed guards. St. Andrews, a gated community in Boca Raton, Florida, spends over \$1 million a year on helicopter and canine patrols« (DILLON 1994: 8). Weitere Merkmale sind ein internes Steuersystem und spezielle Verhaltensregeln für die Bewohner (WEHRHEIM 1999), die über so genannte »homeowner associations« (MCKENZIE 1994: 122) organisiert sind und eine Art Miniaturregierung darstellen. Das zentrale Motiv für das Leben in einer solchen Gemeinschaft ist die Kontrolle (WEHRHEIM 1999), die auf alle Bestandteile der Gemeinschaft einwirkt und die Sicherheit und Stabilität für die Bewohner garantieren soll. Gleichzeitig grenzt sich die Gated Community nach außen ab und reglementiert den Zugang zu den Einrichtungen strikt. Auf diese Weise entstehen in der extremsten Form isolierte Städte, während weniger radikale Variationen sich nur partiell von der Außenwelt abgrenzen. WEHRHEIM (ebd.) sieht in diesen exklusiven Gemeinden eine künftig weiter verbreitete Lebensform, an deren Ende sich der Bewohner zwischen mehreren sicheren 
Zonen bewegt, ohne jemals seine Gated Community zu verlassen. Die Folge wäre einerseits die Konkurrenz zwischen den Communitys und andererseits die Entsolidarisierung der Gesellschaft, da finanzstarke Mitglieder zunehmend in die privaten, sicheren Städte abwandern. In Deutschland gibt es bislang nur eine einzige Gated Community, die sich selbst auch so bezeichnet und im Internet mit »professioneller Dienstleistung« und »friedliche(r) Atmosphäre» (arcadia-potsdam.com) wirbt, »die außerhalb des Zauns nicht möglich erscheint« (ebd.).

Im Zusammenhang von Sicherheit und Freiheit erscheint die Gated Community als Musterbeispiel eines Transfers von Freiheit hin zu gesteigerter Sicherheit. Der Unterschied zur Betrachtung einer größeren Konfiguration ist aber der, dass die Bewohner der Gemeinschaft sich freiwillig für die neue Lebensform entscheiden und diese bewusst leben. Sie können zumindest theoretisch jederzeit ausziehen und woanders weiterleben, was in der größeren Gesellschaft mit wesentlich mehr Aufwand verbunden ist. Im Sinne von Eigenkontrolle geben die Bewohner der Gated Community also Freiheit gezielt und freiwillig auf, während die Mitglieder einer normalen Gesellschaftsordnung dies in der Regel nicht aktiv tun können, da entsprechende Regelungen schon per se vorhanden sind.

Das funktionierende Sicherheitssystem der Gated Communitys sorgt in letzter Konsequenz dafür, dass sich Kriminalität andere, weniger kontrollierte Bereiche suchen muss, was dann die Sicherheit der Anderen reduziert.

\subsection{Sicherheit in der Präventionsgesellschaft}

Die Konsequenz der Eingriffe in die Freiheit ist, wie beschrieben, die Überwachungsgesellschaft. Richtet man den Blick auf die Sicherheit, so lässt sich fest- 
stellen, dass die Bemühungen um Sicherheit immer mehr auf künftige Ereignisse ausgerichtet werden. Damit wird der Boden der Sicherheitsgesellschaft zumindest teilweise verlassen und man versucht sich mit Prävention und Gefahrenabwehr:

»Dass es bis zum heutigen Tag in Deutschland nicht zu Attentaten durch islamistische Terroristen gekommen ist, weil die Vorbereitung von Anschlägen rechtzeitig aufgedeckt wurde, ist der professionellen und umsichtigen Arbeit der deutschen Sicherheitsbehörden, auch des Bundesamtes für Verfassungsschutz, und - gerade im vergangenen Jahr der vertrauensvollen Zusammenarbeit mit Partnerdiensten zu danken. Im vergangenen Jahr haben die Sicherheitsbehörden durch die Festnahme der ıSauerland-Gruppe، vermutlich schwere Anschläge rechtzeitig vereitelt. Die sichergestellten Chemikalien hätten für den Bau von Bomben gereicht, deren Wirkung noch verheerender gewesen wäre als die Anschläge von Madrid und London« (BMI 2007: 3).

Interessant ist hier, dass sowohl der Fahndungserfolg als auch der Fahnungsmisserfolg in der politischen Rhetorik zu verwandten Ergebnissen führen. Die professionelle Arbeit der deutschen Behörden - man beachte, dass auch »Partnerdienste« miteingebunden werden mussten und wurden - war erfolgreich und wurde zu einem Argument für das präventive Vorgehen. Man stelle sich den Misserfolg in Form eines durchgeführten Anschlages vor, bei dem mit Sicherheit der Ruf nach Kompetenzerweiterungen laut geworden wäre, natürlich um künftig verstärkt im Vorfeld tätig werden zu können.

Die Botschaft ist eine einfache. Die Arbeit der Sicherheitsbehörden war bislang erfolgreich, so gut es ging, aber die Folgen des Verbrechens wären von ungeahntem Ausmaß gewesen. Die letzte Konsequenz dieser Denkweise ist eine nicht enden wollende Ausweitung polizeilichen Handelns, die aber niemals zu einem wirklichen Ende kommen kann. Es gibt immer eine weitere, größere Gefahr, der mit immer neuen Maßnahmen präventiv begegnet werden muss. Die möglichen Maßnahmen wiederum entwickeln sich im Einklang mit 
der technischen Weiterent-wicklung, sie werden immer fortschrittlicher und können sich daher ebenfalls nahezu grenzenlos ausweiten. SCHÜLER-SPRINGORUM charakterisiert diesen Zustand äußerst treffend:

»(...) Prävention (stellt) in diesem Sinne eine echte Plattform (dar) (.), eine Startbasis für rechtlich noch relativ ungebundenes, dafür aber buchstäblich sflächendeckendesı Handeln von Polizei. Je mehr diese betont, es werde bloß organisatorisch-technologische (oder gar rationalistisch-wissenschaftliche) Kriminalitätsvorsorge betrieben, desto weniger werden Bürger den Verdacht schöpfen, der Preis der Sicherheit könnte in Freiheit zu zahlen sein. Sie sollten aber.« (SCHÜLER-SPRINGORUM 1991: 117)

Durch diese Entgrenzung der polizeilichen Tätigkeit wird das gesamte Feld der polizeilichen Ermittlung zunehmend intransparenter und komplexer, was sowohl für den Preis gilt, den die Bürger nach SCHÜLER-SPRINGORUM in Freiheit zu zahlen haben als auch für die Evaluation der polizeilichen Arbeit selbst. Es ist kaum einzuschätzen, ob und inwiefern die polizeiliche Arbeit effizient durchgeführt wird, da das Aufgabenfeld immer uferloser wird.

Die Funktionsweise des Präventionsstaates soll nun weitergehend erörtert werden, um letztlich die Auswirkungen auf Sicherheit und Freiheit einschätzen zu können.

\subsubsection{Die Funktionsweise des Präventionsstaates}

Die Antwort auf das oben skizzierte, hypothetische Szenario liegt in der Akzeptanz der veränderten Aufgabe, Prävention wird zum dominanten und bestimmenden Ziel. Das Bundesministerium des Inneren weist in einem Bereich der Kriminalitätsbekämpfung auf seinen Präventionsansatz hin:

„Die Zahl der in Deutschland aktiven islamistischen Organisationen ist im Jahr 2007 auf 30 angestiegen. Und auch die Zahl ihrer Mitglieder und Anhänger ist - einem mehrjährigen Trend folgend - auf nun über 33.000 angestiegen. Diese Entwicklung müssen wir 
aufmerksam verfolgen - auch wenn diese Zahlen nicht mit dem weitaus kleineren Bereich des gewaltbereiten Terrorismus gleichzusetzen sind. Der unverändert hohen Bedrohung durch den gewaltbereiten islamistischen Terrorismus müssen wir mit allen Mitteln des Rechtsstaats entgegentreten. Dabei kommt es vor allem auf eine wirksame Prävention an, damit wir die Täter fassen, bevor sie ihre Gewaltverbrechen ausüben können. Prävention setzt Information voraus. Wenn wir Anschläge verhindern wollen, müssen wir die Handlungsfähigkeit der Sicherheitsbehörden gewährleisten. Dabei kommt es letztlich auf drei Dinge an: auf ausreichendes Personal, auf ausreichende technische Mittel und auf ausreichende Kompetenzen. Es muss gewährleistet sein, dass die Sicherheitsbehörden die notwendigen Informationen gewinnen und sie gegebenenfalls zusammenführen und austauschen können« (BMI 2007: 3).

Bemerkenswert ist hier die Aussage des Innenministeriums, das bereits von Tätern spricht, die gewissermaßen bereits im Vorfeld der Tat zu solchen werden. Insgesamt erinnert der gesamte Ansatz der Prävention an die bekannte Geschichte zweier Männer, von denen einer auf einer Straße steht und alle 10 Sekunden in die Hände klatscht. Auf die Frage seines Begleiters, warum er dies tue, antwortet Ersterer, er mache dies, um die Elefanten zu vertreiben. Auf die Aussage des anderen, er sehe keine Elefanten, die es zu vertreiben gelte, ist die triumphierende Antwort: »Na also! Sehen Sie?« (vgl. u.a. WatZLAWICK 1983: 51f.). WATZLAWICK weist in seinem Kontext darauf hin, dass die Prävention oder das Vermeiden einer bestimmten Situation »scheinbar die vernünftigste Lösung« (ebd.) darstelle, gleichzeitig aber den Fortbestand des Problems garantiere. Um im obigen Islamismus-Beispiel zu bleiben, so kann die Prävention zwar die konkrete Tat unterbinden, allerdings ist nicht ersichtlich, was dies alle Beteiligten kosten wird: Man weiß nicht, wieviel Personal und damit finanzielle Mittel benötigt werden, man weiß, nicht welche Techniken auch künftig benötigt werden und letzlich auch nicht, welche Kompetenzen die Ermittlungsbehörden für die erfolgreiche Prävention künftig noch benötigen werden. 
Doch Prävention ist, wenn man von einer Präventionsgesellschaft spricht, nicht nur als Kriminalprävention zu sehen, sondern als umfassendes Konzept, das eine ganze Palette von gesellschaftlichen Feldern umfasst. STRASSER und SCHWEER sprechen in diesem Zusammenhang von "Prävention in allen Lebenslagen« (vgl. STRASSER/SCHWeER 2008: 165): „Dass Prävention eine gesellschaftliche Aufgabe sei, ist inzwischen in aller Munde - und nicht nur, wenn es um Kriminalität geht. Ob in der öffentlichen Debatte über die Schulreform, das Gesundheitssystem, den Sport, die Alterssicherung, das Sexualverhalten, die Stadtplanung, den Feinstaub, den Katastrophen- oder Virenschutz - überall ist von Prävention die Rede, d.h. von Früherziehung, Prophylaxe, Altersvorsorge, Verhütung, Monitoring, Transparenz, Frühwarnsystemen (...)« (ebd.). Die kommunale Kriminalprävention als wichtigem Bereich der Prävention, so StRASSER und SCHWEer weiter, werde »zum Leitbild für eine neue Sicherheitsordnung« (ebd.). Als Beipspiele für diese benennen Strasser und SCHWEER beispielsweise kriminalpräventive Räte, Ordnungspartnerschaften, Gefahrenabwehrverordnungen, private Sicherheitsdienste, Bürger als Hilfspolizisten usw (vgl. ebd.: 156 f.). Von diesem kommunalen Kontext aus schlagen die Autoren eine Brücke bis hin zum globalen Antiterrorkampf und sprechen von einer so genannten »Präventionskultur« (ebd.: 166). Vor dem Hintergrund der oben stehenden Präventionsbereiche ist dieser Begriff durchaus treffend, zumal sich die Liste noch in anderen Gesellschaftsbereichen fortführen und erweitern ließe.

Die Legitimation zieht die Prävention gewissermaßen aus sich selbst (vgl. ebd.). Gemäß dem Motto «Vorbeugen ist besser als Heilen« erscheint so Prävention auch im Bereich der Kriminalitätsbekämpfung als richtiger Ansatz. Eine Begleiterscheinung dieser Prävention ist eine "gemeinwohl- 
orientiere Orwellisierung" (STRASSER 2005: 51), also völlige Überwachung im Dienste des Vorbeugens.

Kriminalität und Kriminalprävention werden des Weiteren in der Präventionsgesellschaft für politische Zwecke instrumentalisiert (vgl. STRASSER/ SCHWEER 2008: 179), d. h. sie werden zum politischen Inhalt, der Willensbildungsprozessen unterworfen ist. Die entsprechenden präventiven Maßnahmen erwecken als Veränderungen oder Neuerungen auch verstärktes mediales Interesse (vgl. ebd.: 176). Vorbeugende Maßnahmen wirken innovativ und kreativ und können Schlagzeilen produzieren, die sich politisch nutzen lassen, eine Verurteilung eines Einbrechers beispielsweise schafft es mit Müh' und Not gerade noch in den Lokalteil der Zeitung.

Man kann zu Recht von einem grundlegenden Wechsel der Perspektive sprechen, allerdings teilt die Präventionsgesellschaft die Leidenschaft der Überwachungsgesellschaft für Daten. Immerhin ist die Überwachung im Präventionsstaat nicht mehr reiner Selbstzweck, sondern dient jetzt mindestens vorgeblich konkret der Abwehr und Vorbeugung von Gefahren. Eng damit verbunden ist Angst als die bestimmende Motivation. Vor allem als Angst vor Terror-anschlägen ist sie weit verbreitet und wird so zu einer »Autobahn für Sicherheitsgesetze« (PrantL 2002: 17). Sieht man die Angst als in Strukturen ver-festigt an, so kann man in Bereichen der Sicherheitsgesetz-gebung in Anlehnung an GLASSNER (2000) auch von einer regelrechten »Kultur der Angst« sprechen.

Ein internationales Beispiel für den Datenhunger sind die Bestimmungen über den Flugverkehr nach 9/11. Über eine zentrale Datei wurde eine so genannte No-Fly-List erstellt, nach der bestimmte Personen vom Flugverkehr in die Vereinigten Staaten ausgenommen waren, ohne dass die Betroffenen 
eine Möglichkeit hatten, wirksam dagegen vor zu gehen (vgl. BolesCH 2008). Nachweislich waren auch Unschuldige und auch politische Mandatsträger davon betroffen. Insgesamt vervierfachte sich die Zahl der in der USAntiterror-Datenband gespeicherten Personen auf rund 435000 (vgl. RöTZer 2007) Namen, was zu Problemen bei weit verbreiteten Namen und damit zu unliebsamen Erlebnissen, wie das Verpassen von Flügen, führte.

Die Sanktionen durch die Behörden verlagern sich im Präventionsstaat immer weiter in das Vorfeld der Tat. Ein deutsches Beispiel ist neben den schon etablierten anlassungebundenen Kontrollen und Rasterfahndungen auch das Vorhaben des Bundesrates, den Aufenthalt in ausländischen Terrorcamps unter Strafe zu stellen. Als große Gefahr für die innere Sicherheit - die Befürchtung geht wohl von einem unauffälligen Deutschen aus, der sich zum Terroristen ausbilden lässt und dann einem Schläfer gleich sich unbemerkt bis zur Tat in Deutschland bewegt - sollte diese Verhaltensweise strafbar sein, ebenso wie die im Jahre 2002 gekippte Vorschrift, die Gleiches für die »Sympathiewerbung für kriminelle und terroristische Vereinigungen« (BTDS 16/7958) vorsah. Dieser Vorschlag ist in letzter Konsequenz nicht ganz unproblematisch, da in vielen Berufszweige wie Chemie- oder Physiklehrer, den Schützenvereinen oder in Flugschulen auch im Inland auf einfache Weise Erkenntnisse gewonnen werden können, die man zu terroristischen Angriffen problemlos (vgl. u. a. forum.somethingawful.com 2003) benützen könnte. In diesem Sinne droht diesen eigentlich harmlosen Zeitgenossen zukünftig die Kriminalisierung mit entsprechenden negativen Sanktionen.

Die letzte Konsequenz des Präventionsstaates ist eine massive Schwerpunktverlagerung hin zu Sicherheit. HASSEMER fasst die in diesem Zusammenhang wichtigsten Aussagen prägnant zusammen (vgl. HASSEMER 2002: 
$1 \mathrm{ff})$. Den Ursprung der Entwicklung sieht er in den 70 er und 80 er Jahren des letzten Jahrhunderts, verstärkt durch die Anschläge des 11. September 2001, die den Beginn einer Entwicklung im Spannungsverhältnis von Sicherheit und Freiheit hin zu Sicherheit auf Kosten der Freiheit darstellen. Mit den »Stichworten Prävention, Effektivität und Kontrollverschärfung« (ebd.: 2) ließe sich diese Tendenz am besten charakterisieren. HASSEMER konstatiert für den Bereich des materiellen Strafrechts eine kontinuierliche Verschärfung der Strafandrohungen, eine Ausweitung der verbotenen Bereiche und zahlreiche Neu-Kriminalisierungen (ebd.). Ein Beispiel für Letzteres sind Überlegungen, den Besitz von so genannten »Killerspielen« zu kriminalisieren, die immer dann in der öffentlichen Diskussion und in den Anfangsstadien der Gesetzgebung (zum Beispiel auf der Referentenebene in den zuständigen Ministerien) auftauchen, wenn bestimmte Gewalttaten von Jugendlichen begangen werden.

Für den Bereich des formellen Strafrechts, das die Ermittlungsverfahren regelt, stellt HASSEMER eine einseitige Entwicklung hin zu mehr »Effektivität und Verschärfung" (ebd.) fest. Verdachtsunabhängige »Vorfeldermittlungen« (ebd.: 3) würden dem konkreten Verdacht vorgeschaltet und führten letztlich erst zu Verdachtsmomenten. Diese Verdachtserosion (vgl. ebd.) werde ermöglicht durch breit streuende Ermittlungsmethoden, die mit überwiegend »heimlicher Informationbeschaffung " (ebd.) einhergingen: Der Betroffene nehme keinerlei Notiz von »Rasterfahndung, Telefonüberwachung, Lauschangriff oder dem Einsatz verdeckter Ermittler« (ebd.). Die Tendenzen, die Grenzen zwischen den Geheimdiensten, der Strafverfolgung und der Polizei einzuebnen, so HASSEMER weiter, sei eine Begleiterscheinung dieser Entwicklung. In letzter Konsequenz rührten diese Veränderungen nicht von Herrschaftsbedürfnissen des Staates her; sie würden vielmehr von den Kontrollbedürfnissen der Be- 
völkerung und von »einem punitiven, auf Prävention und Risikobeherrschung ausgerichteten Strafklima« (ebd.) getragen. Er schließt seine Ausführungen mit der These, dass es zunehmend schwerer falle, Freiheitsrechte als »Voraussetzungen einer Zivilgesellschaft und als Bedingungen guten Lebens« (ebd.) plausibel zu machen. Stattdessen würden sie als »Stolpersteine auf dem Weg zu einer sicheren Gesellschaft« (ebd.) wahrgenommen.

Den Ausführungen HASSEMERS ist in voller Gänze zuzustimmen, sie charakterisieren treffend die verschiedenen sicherheitspolitischen Entwicklungen der letzten drei Jahrzehnte und die damit einhergehende Ausweitung der Sicherheitsbestrebungen auf Kosten der Freiheit.

\subsubsection{Sachzwänge als wichtigste Bedingung}

Die Präventionsabsicht kann man als typischen Sachzwang interpretieren, der die gesamte politische und gesellschaftliche Diskussion überlagert. Wie so oft fällt es dabei schwer zu unterscheiden, ob dieser Zwang real ist oder nur eine rhetorische Argumentationsfigur (vgl. SCHÜLER-SPRINGORUM 1996: 20), um sich in Entscheidungsprozessen durchzusetzen. Ist die Forderung nach der Speicherung biometrischer Merkmale lediglich ein vorgeschobener Sachzwang oder ist das wirklich sinnvoll? Soll man noch einen Schritt weiter gehen und kurze Filme auf den Pässen abspeichern, die das Identifizieren der Person erleichtern sollen (vgl. KUHLMANN 2008)?

Sachzwänge sorgen für zunehmende Ritualisierung in der Auseinandersetzung, die Angst vor dem Risiko und vor dem Eintreten des schlimmsten Falles wird zur bestimmenden Denkfigur, deren vorgeschobene Versachlichung eine intensive Auseinadersetzung blockiert. Dies zeigt sich beispielsweise 
immer dann, wenn die Medien über eine Kindesentführung oder über Fälle von sexuellem Missbrauch - oftmals auch im Zusammenhang mit kinderpornographischen Bildern auf den Festplatten von Verdächtigen - berichten. Man fordert dann in der Regel harte Strafen für den oder die Täter und gibt den Kindern Verhaltensmaßregeln mit, die deren Aktionsradius einschränken und impft sie gleich noch mit Angst vor dem Fremden. FUREDI spricht in diesem Zusammenhang auch von einer »Elternparanoia« (FUREDI 2007, zitiert nach Steinberger 2007). Die Angst, dem eigenen Kind könne etwas zustoßen, wird irrational und orientiert sich nicht mehr an den tatsächlichen Gegebenheiten, sondern am Risiko. Es werden dabei weder Kosten noch Nutzen abgewogen und in Relation zur Wahrscheinlichkeit gesetzt, dass die befürchtete Situation tatsächlich eintritt. Man mag nun einwenden, dass der Schutz des eigenen Kindes über allem steht und jeden Aufwand rechtfertigt. Problematisch ist aber, dass unter Umständen die falsche Gefahr bestimmend wird - gerade bei sexuellem Missbrauch ist die Angst vor fremden Missbrauchern fehlleitend, da die Täter in der Mehrzahl aus dem sozialen Nahraum (vgl. u.a. BANGE 1992 und BANGE/DEEgenER 1996) der Opfer kommen.

Prävention als alles dominierender Sachzwang wird zum wichtigsten Ziel. Man denkt an elektronische Standortbestimmungen, am besten gleich in Form eines implantierten Computerchips (vgl. Steinberger 2007) und vergisst im gleichen Atemzug, dass auch dieser Schutz nur partiell praktikabel wäre. Welche Botschaft würde man außerdem dem Kind vermitteln? Die Trennlinie zwischen Argumentationsfigur und bloßer Rhetorik scheint an dieser Stelle leicht zu ziehen, es geht ja schließlich um das Wohl des eigenen Nachwuchses. Trotzdem ist weiteres Nachfragen und das Suchen nach alternativen Handlungsmöglichkeiten hier angebracht. Denn zweifellos wird auch das 
Bemühen um umfassende Prävention schon im Vorfeld einige negative Konsequenzen nach sich ziehen.

\subsection{Technik und Daten}

Die bisherigen Ausführungen haben gezeigt, dass bei den verschiedenen Aspekten wie Sicherheit, Freiheit und Prävention immer wieder Daten und neu entwickelte Techniken eine wichtige Rolle spielen. Dies gilt insbesonders, wenn es um Veränderungen derselben geht, die nur zu oft durch neue technische Weiterentwicklungen ausgelöst werden. Gibt es kein Internet, so kann man es auch nicht überwachen. Technische Innovationen wecken Begehrlichkeiten bei den Überwachern und auch neue Hoffnung bei denjenigen, die versuchen, Freiheit und Sicherheit zurückzugewinnen oder auszubauen. Die wichtigsten Beispiele des folgenden Kapitels werden die Antiterror-Datei als Beispiel und Datenbanken ganz generell sein, zusätzlich werden die zu Beginn näher besprochenen technischen Entwicklungen auf ihre Tauglichkeit abgeklopft, mit der man sie in ein Steigern des Grades an Freiheit integrieren kann.

\subsubsection{Technische Entwicklungen}

Im Hinblick auf die Privatheit an sich ist dabei wichtig, dass die hier vorgestellten Techniken weitgehend neutral sind und sich die Frage von gut oder böse nur durch die Anwendung der jeweiligen Technik stellt: »That's because technology by itself doesn't violate our privacy or anything else: it's the people using this technology and the policies they carry out that create violations" (GARFINKEL 2001: 5). Im Jahr 1972 prognostizierten WESTIN und BAKER 
mehrere technische Entwicklungen, die sie vor ihrem theoretischen Hintergrund einer freien Gesellschaft einzuschätzen versuchten (WESTIN/BAKER 1972: $324 \mathrm{ff}$.) und die alle realisiert wurden und teilweise schon wieder als überholt gelten können:

- Input: Optische Scanner und elektromagnetische Laufwerke (keine Lochkarten mehr);

- Stark anwachsender Massenspeicher;

- Bessere Konfigurationsmöglichkeiten, Netzwerke;

- Verlässliche Systeme;

- Verbesserte Betriebssystemsoftware mit integrativen Ansätzen;

- Größere Verfügbarkeit von Computern;

- Verbesserte Speicherung.

Die Autoren gehen von einer weiten Verbreitung von Computern aus und vor allem auch von Austauschmöglichkeiten zwischen den einzelnen Benutzern (vgl. ebd.: 331). Die Folgerungen sind knapp formuliert und eindeutig. Sie rechnen damit, dass mit der Verbesserung der Computerleistung automatisch immer mehr personenbezogene Daten gespeichert werden und dass diese auch vermehrt ausgetauscht werden. Die Folge wird daher sein, dass es schwerer werden wird, seine sensiblen Daten zu schützen und es wird wegen ihres Volumens und der redundanten Speicherung an verschiedenen Orten, fast unmöglich werden, Zugriff auf die gespeicherten Daten zur Gänze zu bekommen.

Obwohl sie nicht direkt als Computer einzustufen ist, vereint die sogenannten RFID-Technik alle von WESTIN/BLAKE angenommenen Entwicklungsmerkmale. Sie steht für Innovation, Miniaturisierung und neue Funktionen. Beim RFID handelt es sich um eine »Datenübermittlung mittels Funksignalen« 
(EISEnberg U.a. 2005: 94), die nach SCHAAR eine »Schlüsselkomponente unseres Alltags« (SCHAAR 2006: 41) ist. Ungebunden von physischen Barrieren macht sie alle Bürger ein Stück weiter kontrollierbar. Besonders problematisch ist die Distanz, über die zum Beispiel Daten von Reisepässen ausgelesen werden können (vgl. ebd.) oder auf denen auch Informationen über den Inhaber von Fussballtickets der Weltmeisterschaft im Jahre 2006 gespeichert waren (ebd.). Die Funkchips verrichten in Supermärkten ihren Dienst, was von den beteiligten Firmen besondere Transparenz fordert: Dem Verbaucher muss die Möglichkeit bleiben, die Funkchips zu erkennen und zu deaktiveren, da sie bei Verlassen des Ladens weiter funktionieren.

Die besondere Missbrauchsgefahr liegt auch in einer Ausweitung der Speicherkapazität, die es ermöglicht, eine ganze Menge an persönlichen Informationen darauf speicherbar zu machen und diese dann der Möglichkeit des unbemerkten Zugriffs auszusetzen. Für den Umgang mit dieser potenziell gefährlichen Technologie, stellte die Konferenz der Datenschutzbeauftragten die folgenden Forderungen auf: Alle Betroffenen müssen umfassend über den Einsatz, Verwendungszweck und Inhalt von RFID-Chips informiert werden (Transparenz ). Nicht nur die eingesetzten RFID-Chips selbst, sondern auch die Kommunikationsvorgänge, die durch die Chips ausgelöst werden, müssen für die Betroffenen leicht zu erkennen sein. Eine heimliche Anwendung darf es nicht geben (Kennzeichnungspflicht). Daten von RFID-Chips aus verschiedenen Produkten dürfen nur so verarbeitet werden, dass personenbezogene Verhaltens-, Nutzungs- und Bewegungsprofile ausschließlich mit Wissen und Zustimmung der Betroffenen erstellt werden können. Soweit eine eindeutige Identifizierung einzelner Gegenstände für einen bestimmten Anwendungszweck nicht erforderlich ist, muss auf eine Speicherung eindeutig identifizie- 
render Merkmale auf den RFID-Chips verzichtet werden. Damit ist die heimliche Bildung von Profilen nicht erlaubt. Das unbefugte Auslesen der gespeicherten Daten muss beispielsweise durch Verschlüsselung bei ihrer Speicherung und Übertragung unterbunden werden. Es muss vor allem im Handels- und Dienstleistungssektor die Möglichkeit bestehen, RFID-Chips dauerhaft zu deaktivieren, bzw. die darauf enthaltenen Daten zu löschen, insbesondere dann, wenn Daten für die Zwecke nicht mehr erforderlich sind, für die sie auf dem RFID-Chips gespeichert wurden (vgl. SCHAAR 2007: 41).

Da das Internet für die Kommunikation immer wichtiger wird, sollen im Folgenden noch kurz einige Anmerkungen zu wichtigen technischen Aspekten gemacht werden. Die bereits erwähnte Netzstruktur ergibt sich aus dem Zusammenspiel von Client und Server einerseits (vgl. SCHWENK 2002: 2) und der Kommunikation zwischen den Servern über diverse Knotenpunkte andererseits. Auf Grund der ursprünglich militärischen Konzeption des Netzes werden Daten, die unter der Verwendung von Transferprotokollen übermittelt werden sollen, aufgespalten und über viele verschiedene Knotenpunkte übertragen, so dass eventuelle Ausfälle kompensiert werden können. Aufgrund dieser Struktur sind die einzelnen Datenpakete über das ganze Netz verteilt unterwegs und können abgefangen werden. Verschlüsselung oder Kryptographie bietet aus dieser Situation einen Ausweg, indem versucht wird, die Daten für Unbefugte unlesbar zu machen: „Kryptographie ist eine öffentliche mathematische Wissenschaft, in der Vertrauen geschaffen, übertragen und erhalten wird« (Beutelspacher u.a. 2005: 1). Die Ursprünge dieser »Kulturtechnik« (vgl. RösCH 2005: 1) des Verschlüsselns liegen im militärischen Bereich und bestanden anfänglich aus dem Verbergen von Nachrichten und einfachen Codierungen, die immer wieder entschlüsselt wurden. Einer der bekanntes- 
ten, neueren Verschlüsselungen war die der deutschen Enigma, deren Entschlüsselung (vgl. SINGH 2001: 123 ff.) den Ausgang und die Dauer des Zweiten Weltkrieges beeinflusste. Ein besonderes Problem bei jeder Art von verschlüsselter Kommunikation war die Übergabe des Codes, mit dem der Adressat die Botschaft wieder entschlüsseln konnte: Wurde er abgefangen, so war die Nachricht geknackt, gewissermaßen durch eine Art des »social enginieering«. Das so genannte Schlüsselverteilungsproblem wurde schließlich von drei Mathematikern (vgl. ebd.) gelöst, die ein Verfahren ersannen, mittels eines asymetrischen Schlüssels, der aus einem privaten und einem öffentlichen Schlüssel bestand, die Nachricht zu verschlüsseln.

Diese geniale Entdeckung bereitete der Public-Key-Kryptographie (vgl. BEUTELSPACHER 2002: 93) den Weg, der heute der Verschlüsselungsstandard für E-Mailverschlüsselung ist. Das System, das aus diesem Verfahren entwickelt wurde, nannte sich PGP - Pretty Good Privacy. Es wurde zunächst seinem Schöpfer Phil ZimmermanN zum Verhängnis, der sich durch die Verbreitung nach amerikanischem Recht strafbar gemacht hatte (vgl. ZIMMERMANN 1999). Nach einiger Zeit und der erfolgreichen Verbreitung des Verfahrens durch das Internet, wurden die Ermittlungen gegen den Schöpfer von PGP eingestellt. Es ist auch heute der zentrale Verschlüsselungsstandard für Emailverkehr und Online-Einkäufe und schützt immer noch zuverlässig. Da bislang alle Verschlüsselungen früher oder später geknackt worden sind, wird dies mit großer Wahrscheinlichkeit auch mit der RSA-Verschlüsselung geschehen, die PGP benutzt. Die schneller werdenden Computer unterstützen immer mehr auch Codebrecher, die beispielsweise mittels eine Brute-Force-Methode Kombinationen in schneller Reihenfolge - erfolgreich bei Passwörtern von Emailaccounts, die ein geringeres Sicherheitsniveau als PGP besitzen - 
ausprobieren. Die Codierer suchen im Bereich der Quantenkryptographie neue und sichere Methoden (vgl. SINGH 2001: 270 ff.).

\subsubsection{Datenbanken und Kontrolle}

Datenbanken sind heute Schlüsseltechnologien, welche die besondere Bedeutung der Technik und der Datenquantität deutlich werden lassen. Datenbanken sind zunächst ganz einfach Systeme, die auf elektronischem Wege Daten verwalten. Dementsprechend bestehen sie aus zwei Komponenten, nämlich dem Speicherort der einzelnen Datensätze und einer Verwaltungssoftware, die jeden Datensatz eindeutig definiert und auffindbar macht. Das Einsatzgebiet ist riesig. Ob in Notenprogrammen für Lehrer, bei der Ausleihe eines Buches über ein Bibliothekssystem oder beim Ansurfen einer komplexeren Webseite: Fast ohne Ausnahme bewegt man sich im Umfeld von Datenbanken. Datenbanken kann man, wenn sie entsprechend kompatibel sind, zusammenführen. Die Folge ist, dass man Zugriff auf große Datenmengen bekommen kann, wie das Beispiel Antiterror-Datei (siehe Abbildung 17) zeigt, unter der auf viele verschiedene Datenbanken zugegriffen werden kann. Durch die Fortschritte in der Computertechnik ist der Zugriff auf solche Datenbanken bislang immer schneller geworden. Setzen sich künftig Computer mit mehreren Prozessorkernen durch, die wie geschaffen für das Ausführen paralleler Arbeitsschritte sind, wird dieser Performancezuwachs sich weiter beschleunigen, zumal heute verstärkt auf Kompatibilität geachtet wird und so auch die Software ihren Teil beiträgt. Die besondere Gefahr für die Freiheit sind die fast schon unbegrenzten Möglichkeiten, Daten aus unterschiedlichen Quellen zeitnah zu kombinieren, wobei es kaum zu kontrollieren ist, welche Merkmale abgefragt werden. Eventuell vorhandene Logdateien, die dies leisten könnten, sind gleichfalls 
Abbildung 17 In der Anti-Terror-Datei zusammengeführte Datenbanken (Auszug, vgl. BTDS 16/2875, 6.10. 2006)

\begin{tabular}{|c|c|c|}
\hline Name & Datensätze & Zweck \\
\hline AFIS-P & 3300000 & Personenidentifizierung \\
\hline APOK & 281240 & Organisierte Kriminalität \\
\hline APR & 538503 & Straftaten nach BtmG \\
\hline Auswertung RG & 397563 & Organisierte Rauschgiftkriminalität \\
\hline DEO (DOK-EUROPA-OST) & 463447 & Eigentumskriminalität \\
\hline Fingerabdrücke $-\mathrm{A}$ & Gesamt & Fingerabdrücke Asylantragsteller \\
\hline Fingerabdrücke $-\mathrm{P}$ & 1289806 & Finger/Handflächenabdrücke Pol/Bpol/Zoll/BKA \\
\hline DNA-Datei & 1018815 & Vorsorge DNA-Datei \\
\hline DOMESCH & 116910 & $\begin{array}{l}\text { Bekämpfung Dokumenten/Menschenhandel/ Schleusungs- } \\
\text { kriminalität }\end{array}$ \\
\hline Erkennungsdienst & 5786990 & Erkennungsdienstlich behandelte Personen \\
\hline FALSCHGELD & 1058600 & Falschgeldkriminalität \\
\hline FDR & 740257 & Falldatei Rauschgift \\
\hline FEDOK & 7435 & Finanzermittlungsdokumentation \\
\hline FIT & 13912 & Fundstellennachweis islamistischer Terrorismus \\
\hline FUSION & 38644 & Rockerkriminalität \\
\hline FUZ & 16214 & Fälschung unbarer Zahlungsmittel \\
\hline GELDWÄSCHE & 255881 & Geldwäsche \\
\hline GEW LINKS & 1292 & Gewalttäterdatei Phänomenbereich »links« \\
\hline GEW PS & 60 & Gewalttäterdatei Personenschutz \\
\hline GEW AUSLAND & 298 & $\begin{array}{l}\text { Gewalttäterdatei Phänomenbereich »politisch motivierte } \\
\text { Ausländerkriminalität« }\end{array}$ \\
\hline GEW SPORT & 9284 & Gewalttäterdatei Phänomenbereich Sport \\
\hline HAFTDATEI & 99664 & Personen in behördlichem Gewahrsam \\
\hline INNERE SICHERHEIT & 1451605 & $\begin{array}{l}\text { Aufklärung politisch motivierter Straftaten mit länder- } \\
\text { übergreifender internationaler Bedeutung }\end{array}$ \\
\hline KAN & 3591360 & Kriminalaktennachweis \\
\hline KFZ & 2503 & KFZ-Kriminalität \\
\hline KINDERPORNOGRAFIE & 318537 & - \\
\hline KORRUPTION & 7372 & - \\
\hline NSIS-P & 1266826 & Fahndung nach Personen \\
\hline NSIS-S & 15548506 & Fahndung nach Sachen \\
\hline PERSONENFAHNDUNG & 865296 & - \\
\hline SACHFAHNDUNG & 10600000 & - \\
\hline VERMI/UTOT & 30491 & Vermisste und unbekannte Tote \\
\hline VICLAS & - & $\begin{array}{l}\text { Violent Crime Linkage Analysis, Analysesystem zur } \\
\text { Verknüpfung von Erkenntninssen }\end{array}$ \\
\hline
\end{tabular}


Abbildung $17 \quad$ 1. Fortsetzung

\begin{tabular}{|c|c|c|}
\hline Name & Datensätze & Zweck \\
\hline Geschw. & 653744 & Geldwäsche \\
\hline Geschw. & 653744 & Mord \\
\hline Geschw. & 13358 & Prostitution und Zuhälterei \\
\hline Geschw. & - & Landesverräterische/geheimdienstliche Agententätigkeit \\
\hline Geschw. & 257 & Falschgeldverbreitung \\
\hline Geschw. & 11540 & Verstoß gegen $\S 129 \mathrm{~b}$ StGB \\
\hline Geschw. & 9440 & Computersabotage und Datenveränderung \\
\hline Geschw. & 223773 & $\begin{array}{l}\text { Verdacht der Mitgliedschaft einer terroristischen Vereinigung in } \\
\text { Tateinheit mit Mord und Angriffen auf den Luftverkehr }\end{array}$ \\
\hline$A B C$ & 3923 & Alle bekannt gewordenen Fälle der ABC-Kriminalität \\
\hline ABC-Netzwerk & - & $\begin{array}{l}\text { Alle nationalen und internationalen Dienststellen mit } \\
\text { ABC-Fachkenntnissen }\end{array}$ \\
\hline AFIS-A & 900000 & Personenidentifizierung Asylbewerber \\
\hline AUSWERTUNG SEXTOURISTEN & - & Kindermissbrauch im Ausland \\
\hline BALKAN & - & Rauschgiftschmuggel über die Balkanroute \\
\hline BELLA MACHINA & - & KFZ-Kriminalität Deutschland-Italien \\
\hline BILDDATENBANK KINDERPORNOGRAFIE & - & Bilddatenbank Kinderpornografie \\
\hline BKA-AN & 2363378 & BKA-Kriminalaktennachweis \\
\hline BULGARIEN & - & Geldscheinfälschung mit Herkunft Bulgarien \\
\hline CAMOUFLAGE & - & Illegaler Umbau von Waffen, High Impact Procect der EU \\
\hline CONTAINER & - & Bekämpfung Drogenschmuggel per Seecontainer \\
\hline DABIS & $\begin{array}{l}21197 \text { Personen } \\
3749 \text { Organisationen }\end{array}$ & Islamistischer Terrorismus \\
\hline DAREX & - & Druckerzeugnisse, CD, DVD etc. politisch motivierte Kriminalität \\
\hline FIU & 196475 & Sammlung und Auswertung von Verdachtsanzeigen \\
\hline FLASH & - & Eigentumskriminalität osteuropäischer Tätergruppen \\
\hline BKA FALSCHGELD & - & Falschgeldkriminalität \\
\hline BKA GEISELNAHME-ERPRESSUNG-RAUB & - & Geiselnahmen, Erpressung und Raub \\
\hline BKA WAFFEN & - & Straftaten gegen das Waffengesetz \\
\hline BKA TÖTUNGS- und SEXUALDELIKTE & - & Straftaten gegen das Leben und die körperliche Unversehrtheit \\
\hline G 8 & 162 & Informationen zum G8-Gipfel in Heiligendamm \\
\hline GALAXY & - & Bekämpfung des Warenkreditbetruges \\
\hline GENOZID & - & $\begin{array}{l}\text { Datei der Zentralstelle für die Bekämpfung von } \\
\text { Kriegsverbrechen }\end{array}$ \\
\hline IgaSt & - & $\begin{array}{l}\text { International agierende gewaltbereite Störer } \\
\text { (Globalisierungsgegner) }\end{array}$ \\
\hline InTE-Z & $\begin{array}{l}7762 \text { Objekte } \\
17320 \text { Beziehungen }\end{array}$ & Bekämpfung des internationalen Terrorismus/Extremismus \\
\hline
\end{tabular}


Abbildung $17 \quad$ 2. Fortsetzung

\begin{tabular}{|c|c|c|}
\hline Name & Datensätze & Zweck \\
\hline LANDESVERRAT & 176250 & Landesverrat, Gefährdung der äußeren Sicherheit \\
\hline MALATOK & - & Bekämpfung des Menschenhandels \\
\hline OPERATION BALENO & - & Kinderpornografie im Internet (NL) \\
\hline OPERATION CANDYMAN & - & Kinderpornografie im Internet (USA) \\
\hline OPERATION FALCON & - & Kinderpornografie im Internet (USA) \\
\hline OPERATION ICEBREAKER & - & Kinderpornografie im Internet (It) \\
\hline OPERATION NIEVE & - & Kinderpornografie im Internet (E) \\
\hline OStrA & - & Bekämpfung osteuropäische Straftäter \\
\hline PERSONENLISTE ST-32 & - & $\begin{array}{l}\text { Übersicht Gefährder/Beschuldigte/Grundinformationen } \\
\text { islamistischer Terrorismus }\end{array}$ \\
\hline ReKa & - & Rechtsextreme Kameradschaften \\
\hline SEStrA & - & Zentralstellendatei Sexualstraftäter \\
\hline SUNRISE & - & Offene Haftbefehle Fahndungsregion Thailand \\
\hline TANFOGLIO & - & Umbau von Schreckschusspistolen zu scharfen Waffen \\
\hline TATMITTELMELDEDIENST & - & Datei über Sprengstoff- und Branddelikte \\
\hline TIMESHARING & 6525 & Timesharing-Straftaten \\
\hline TURKISH SYDRO CONNECTION & - & Datei zur Bekämpfung synthetischer Drogen \\
\hline VERIMOUNT & - & Sammlung und Auswertung zur Wirtschaftskriminalität \\
\hline TEC & 20768 & Sammlung und Auswertung illegaler Technologietransfer \\
\hline WAFFEN & 23879 & Sammlung und Auswertung Waffenhandel \\
\hline BAN & - & Bundespolizeiaktennachweis \\
\hline PAVOS-Zentral & - & $\begin{array}{l}\text { Polizeiliche Vorgangsbearbeitung, el. Tagebuch der } \\
\text { Bundespolizei }\end{array}$ \\
\hline GGFG & - & Geschützter Grenzfahndungsbestand \\
\hline TaFaS & - & Taschendiebstahlsfahndungssystem \\
\hline EAO Video Bahn & - & $\begin{array}{l}\text { Einrichtungsanordnung zur automatisierten offenen } \\
\text { Videoüberwachung von Bahnanlagen }\end{array}$ \\
\hline INZOLL & - & Informationssystem Zollstraftaten \\
\hline EURIS & - & $\begin{array}{l}\text { Erfassungs- und Recherchesystem der Informations- } \\
\text { gewinnungssachgebiete des Zolls }\end{array}$ \\
\hline VHG & - & $\begin{array}{l}\text { Verdachtshinweise Geldwäsche von Bankinstituten und } \\
\text { Spielbanken }\end{array}$ \\
\hline COLUMBUS & - & Informationsdatei Verbrauchssteuerhinterziehung \\
\hline CARGO-INFO & - & Analysesystem Luftfracht, Express, Kurier- und Luftpostverkehr \\
\hline MAR/YACHT-INFO & - & Analysesystem gewerblicher/nichtgewerblicher Seeverkehr \\
\hline BALKAN-INFO & - & Analysesystem Balkanroute \\
\hline SAMBA & - & Sammlung Meldungen und Berichte Außenwirtschaft \\
\hline KOBRA & - & Kontrolle bei der Ausfuhr \\
\hline
\end{tabular}


manipulierbar und somit nicht vertrauenswürdig. Eine besondere Schwäche des Prinzips Datenbank ist gerade auch die Automatisierung, bei der auch zu viele Daten sinnlos kombiniert werden und somit zu einem riesigen Datenberg führen können, der dann eher den untersuchenden Blick verstellt als erhellt. Auch die eigene Nutzung einer Datenbank durch einen Einzelnen kann negative Folgen haben, wie das Beispiel SCHUFA zeigt. Mitunter kamen Fälle vor, bei denen der Scoringwert der SCHUFA durch eine bloße Kreditanfrage auf rein theoretischer Basis zu einer Verschlechterung des Scoringwertes und dadurch auch zu schlechteren Kreditkonditionen führte. Die Benutzer des Systems wurden durch eine eigentlich neutrale bis positive Handlung (Planung und Vorausschau) mit negativen Merkmalen automatisch registiert.

\subsubsection{Freiheit zurückgewinnen}

Besteht somit überhaupt die Möglichkeit, verloren gegangene Freiheit zurück zu gewinnen? Für GARFINKEL wäre es fatal für jede freie Gesellschaft, wenn sie darauf verzichten würde, ihre Privatheit und damit auch ihre Freiheit zurück zu fordern, sie ist für ihn das fundamentale Recht schlechthin:

» Without the ability to prevent or control intrusions, life itself cannot exist. Simple organsims use their cell walls to protect their (integrity) from intrusions. We humans rely on our skin, our homes, our fences, and our weapons to protect our integrety and our privacy.

- Without privacy of thought - the freedom that allows us to form our own opinions, and the secrecy that allows us to keep our opinions private until we choose to reveal them - there can be no individuality.

- Without privacy of communications, there can be not politics and ultimately no relationships. People can't have honest discussions with one another if they think their words are being overheard and possibly recorded. Just a privacy is a fundamen- 
tal requirement for the development of the self, privacy between individuals is a fundamental requirement for the creation of true and lasting relationships« (GARFINKEL 2001: 257 f.)

Die drei Bestandteile von GARFInKELS Freiheit sind recht basal. Er geht von der physischen Integrität aus, die am eigenen Körper anfängt und sich auf die eigenen Räumlichkeiten ausweiten lässt. Darauf setzt die Freiheit der Gedanken auf, die er als sehr bedeutsam einstuft (ebd.). Er zählt dazu die Meinungsfreiheit, aber auch die Möglichkeit, die eigene Meinung nicht öffentlich machen zu können - für ihn ist das die Basis der Individualität. Die Privatheit der Kommunikation ist ein weiterer Bestandteil, der garantieren soll, dass sich auf Basis der Vertraulichkeit zwischenmenschliche Beziehungen überhaupt entstehen können.

Auf der Basis dieser drei Aspekte kann man Überlegungen anstellen, wie es gelingen kann, Freiheit zumindest auf theoretischer Basis zu restaurieren. Der Schutz der eigenen vier Wände kann nur durch rein baulich-technische Maßnahmen erreicht werden, nämlich durch das Absichern der Fenster und Mauern gegen Abhörmaßnahmen von außen. Wanzensuchgeräte können helfen, auch passiv betriebene Störenfriede zu entdecken. Sämtliche Kommunikationsformen, die das eigene Heim mit der Außenwelt verbinden sollte man auf Grund des derzeitigen technischen und rechtlichen Standes über das Internet abwickeln. Ausgehend von unbenutzter Hardware kann man ein intaktes System aufsetzen, das anschließend jede Kommunikationsform verschlüsselt abwickelt. Voraussetzung ist dabei, dass man ein alternatives Betriebssystem wie Mac OS X oder Linux benutzt und zusätzlich - will man ganz sicher gehen das Surfen im Internet über so genannte virtuelle Maschinen abwickelt, die man nach der Benützung leichter bei Bedarf löschen oder zurücksetzen kann. 
Durch diese Virtualisierung lässt sich effektiv unterbinden, dass sich Schadsoftware auf der Ebene des Betriebssystems installiert. Die regelmäßige Pflege der eigentlichen Systemsoftware mit Hersteller-Updates ist ebenfalls extrem wichtig. Eine hilfreiche Ergänzung sind dabei Programme wie PGP für die Emailverschlüsselung und das Verwenden von Proxyservern zur Verschleierung der eigenen IP-Adresse, über die man sonst identifizierbar wird. Das Benützen eines TOR-Netzwerkes kann Spuren im World Wide Web zuverlässig verwischen. Datenverschlüsselung aller Dateien ist das oberste Gebot und kann in aktuellen Betriebssystemen bereits automatisch betrieben werden. Auch auf das Handy muss man nicht verzichten, es bieten sich dafür Kartentauschdienste an, die ein beliebiges Prepaidhandy in bestimmtem Maße anonymisieren können (vgl. daten-speicherung.de 2008). Die Voice-over-IP-Technologie ist momentan noch nicht Bestandteil von Abhörlösungen und daher ebenfalls als sicher einzustufen.

Außerhalb der eigenen vier Wände ist es wesentlich schwerer, der Fremdkontrolle auszuweichen. Das Meiden von überwachten Plätzen ist mittlerweile fast unrealisierbar und wäre auch kein besonderes Zeichen von Freiheit: Sogar für den Zutritt zu Räumen bestimmter Universitäten und zum Ausleihen eines Videofilmes wird heute bei vorhandenen Zutrittssystemen mitunter die Hinterlegung eines Fingerabdruckes gefordert.

Zusätzlich zu den oben genannten individuellen Verhaltensweisen kann Freiheit auch im Rahmen politischen Handelns und durch Pflege der demokratischen Kultur zurückgewonnen werden. Besondere Beachtung sollten dabei strukturelle Schwächen des politischen Systems finden. Gängige Vorstellungen vom System der deutschen Gewaltenteilung gehen von einem klassischen dreiteiligen Aufbau aus (siehe Abbildung 18). 


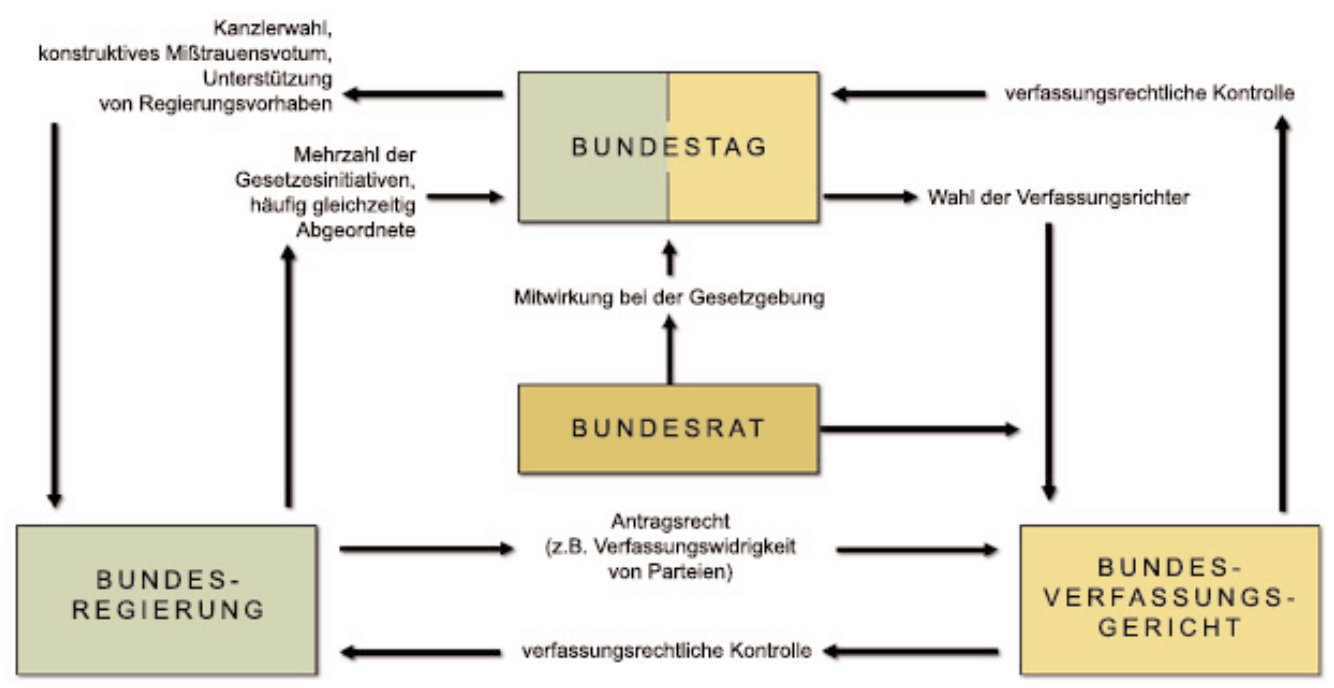

Abbildung 18 Gewaltenteilung in der Theorie (bund.de 2008, zitiert nach HOCHSCHILD 2005)

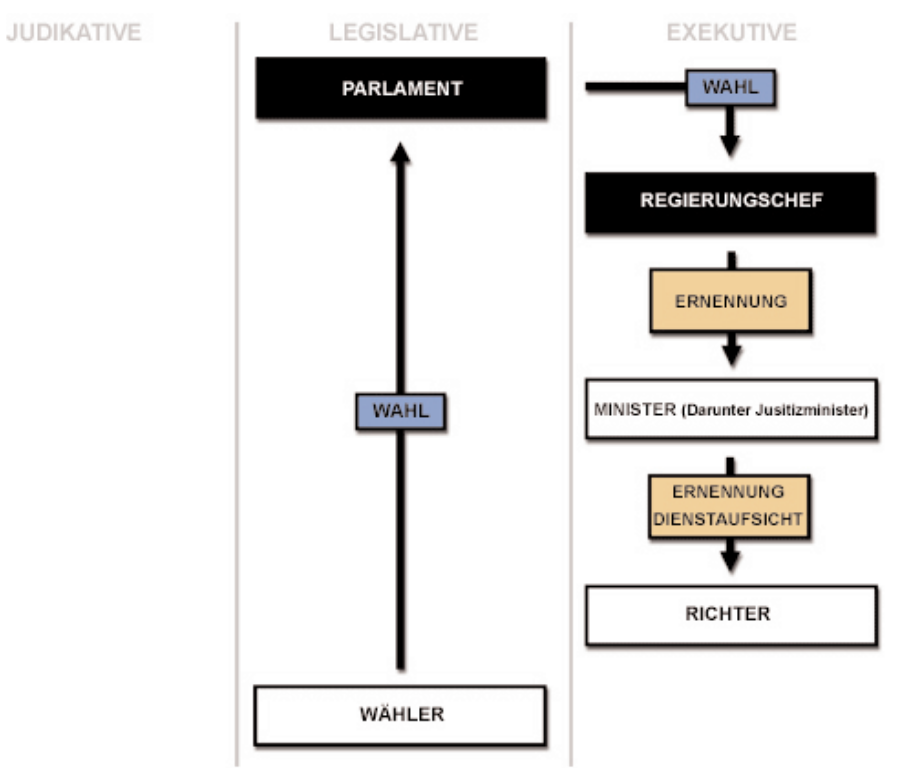

Abbildung 19 Gewaltenteilung in der politischen Realität (HOCHSCHILD 2005) 
Im Gegensatz dazu existieren aber auch andere Sichtweisen. Berücksichtigt man die Abhängigkeiten der Richter unterhalb der Ebene der obersten Gerichte und des Bundesverfassungsgerichtes, so offenbart sich anstelle des gewohnten Bildes ein abweichendes Schema (siehe Abbildung 19), das nun nicht mehr von einer eigenständigen judikativen Gewalt ausgeht. An Stelle der ohnehin nicht ganz korrekt aufgezeichneten Kontrolle der Bundesregierung durch das Bundesverfassungsgericht, die so nicht stattfindet, da nur die Gesetzesakte des Bundestages und nicht das Handeln der Bundesregierung kontrolliert wird, untersteht im zweiten Schaubild der ganze Unterbau der Justiz der Exekutive, da die Richterernennung und Finanzierung in den Händen der Justizminister liegt. Gerade im Hinblick auf die vielen Richtervorbehalte, die exekutive Maßnahmen betreffen, wäre es mehr als sinnvoll, wenn die Richter auf den unteren und mittleren Ebenen des Rechtssystems tatsächlich nicht ein Mitglied der Exekutive als Vorgesetzten hätten.

Auch der Blick nach Brüssel kann lohnen, da über die europäische Kommission ein Gesetzgeber tätig ist, der sehr sachorientiert und zum Wohle Gesamteuropas arbeiten muss. Ein politisches wichtiges Ziel kann es sein, sowohl in Brüssel als auch in Berlin für mehr Transparenz und der Verbesserung der Möglichkeit einzu-treten, mehr Einblick in persönlich relevante Akten aller Behörden zu bekommen (vgl. SCHuLzKI-HAdDoutI/REDELFS: 180). Der persönliche Kontakt zu den Volksvertretern auf allen politischen Ebenen in Land, Bund und Europa ist eine wichtige Gelegenheit, auf die Defizite aufmerksam zu machen und sich an der politisch notwendigen Diskussion zu beteiligen. 


\subsection{Zusammenfassung und Bewertung}

Eine Vielzahl an neu eingeführten gesetzlichen Regelungen haben den Grad an Freiheit - besonders im Bereich der individuellen Eigenkontrolle - sinken lassen. Sowohl der private Raum und die Öffentlichkeit als auch die Kommunikationsschnittsttellen sind von Fremdkontrolle und Überwachung bedroht. Das Recht auf informationelle Selbstbestimmung wird dabei von der Mehrheit aller Beteiligten gering geachtet. Es herrscht allgemein ein zu laxer Umgang mit persönlichen Daten, die von vielen verschiedenen Stellen erhoben und gespeichert werden. Auszumachen ist dabei nicht unbedingt ein staatlicher Big Brother, sondern eine enorme Anzahl kleiner Geschwister, die andere überwachen und denen die moderne Kommunikationstechnik es ermöglicht, ihr Innerstes selbst nach außen für andere zu inszenieren. Es ist nicht verkehrt, von einem radikalen Überwachungs- und Veröffentlichungspluralismus zu sprechen: Viele überwachen und viele veröffentlichen von sich aus, staatliche Behörden sind nur einer der beteiligten Akteure. Eine wichtige Frage ist nun, ob für die Betroffenen die Möglichkeit besteht, Freiheit zumindest graduell zurück zu erlangen.

\subsubsection{Freiheit durch Gegenmaßnahmen?}

Das bewusste Benützen dieser Mechanismen, etwa durch eine Veröffentlichung eigener Informationen über einen Blog, ist ein Ausdruck von (Meinungs-)freiheit. Den wenigsten Menschen ist aber bewusst, dass heutige Medien und das Internet nach dem Prinzip der mehrfachen Redundanz arbeiten: Es wird archiviert, auf neudeutsch gebackupt, »bis der Arzt kommt», 
allerdings handelt es sich dabei in der Regel um Datensicherungen ohne formationelle Rechteinhaber. Längst überholte politische Meinungen, nicht mehr angemessene fotographische Motive und Hobbys, eine politische Diskussion in einem Forum, eine unpassende Kreditkartenabrechnung und eine schier unendliche Anzahl an weiteren Informationen bleiben dauerhaft gespeichert und abrufbar. Und selbst wenn Bewusstsein für die Problematik entsteht und Einzelne begreifen, dass Gegenmaßnahmen ergriffen werden können, dann wird schnell offensichtlich, dass alle Gegenmaßnahmen aufwändig sind, Disziplin erfordern und teilweise technisch für den Normalbürger kaum zu bewältigen sind. Im Rahmen dieser Forschungsarbeit wurde in einer Art Miniaturfeldversuch - allerdings ohne entsprechendes experimentelles Design mit Kontrollgruppen oder Ähnlichem, sondern gewissermaßen nur als isolierte, nichtformalisierte Beobachtungen - versucht, mehrere Privatsphären möglichst sicher zu machen. Kann man sich selbst so schützen, wie es Roland Appel so plakativ beschrieben hat?

\footnotetext{
»Ähnlich wie im 'Wilden Westen' hilft es auf absehbare Zeit nur, uns selbst zu schützen. Der Colt ist in diesem Falle ein möglichst sicherer Browser, Software und Betriebssysteme, die nur schwer von Dritten ausspioniert werden können. Dabei hat derzeit die einzige wirklich kostenlose Linux/Open-Source-Software die vermutlich größte Sicherheit zu bieten. Dazu ein guter Virenschutz und allem voran: Wissen und Bildung. Wissen ist Macht, das gilt im Internetzeitalter in verschärfter Form. Wem heute Wissen fehlt, wird morgen immer machtloser sein (APEL 2008, zitiert nach BORCHERS 2008)
}

In der Praxis kristallisierte sich ziemlich schnell heraus, dass dies mit einer Vielzahl an günstigen Mitteln innerhalb der eigenen vier Wände theoretisch möglich war, besonders für den Bereich der computergestützten Kommunikation. Der APPEL'SCHE Colt zum Selbstschutz war günstig zu bekommen, aber er bedarf auch gründlicher Pflege, damit er »nicht nach hinten« losgeht. Als 
große Schwachstelle entpuppte sich dabei in der Tat das Betriebssystem des Computers und die wichtigste Schnittstelle, nämlich die von Mensch und Maschine. Innerhalb von wenigen Tagen war die Disziplin zum Schützen der eigenen Sphäre wieder in Vergessenheit geraten und sogar elementare Dinge wurden vergessen. Verschlüsselte Kommunikationsformen erwiesen sich als zu hinderlich, da entsprechende Maßnahmen auch passende Kommunikationspartner benötigen, die es zunächst einmal zu überzeugen gilt. Anonymisiertes Surfen mittels des TOR-Netzwerks erwies sich in der vorhandenen Computerkonfiguration als äußerst langsam mit einem durchschnittlichen DSL 2000-Anschluss, der mittlerweile als gefühlter Standard für viele Haushalte gilt. Chatsysteme ohne jegliche Verschlüsselungsmöglichkeiten und das Pflegen der sozialen Netzwerke erwiesen sich nach wie vor als verführerisch und auch das Benützen alternativer Suchmaschinen jenseits von Google konnte nicht durchgehalten werden: Zu oft sind entsprechende Suchmasken in die Browser eingebunden und Alternativen verstecken sich in den Untiefen der Programmmenüs und -einstellungen.

Eine Onlinedurchsuchung wäre vermutlich überhaupt kein Hindernis gewesen, zumindest hätte das Einschleusen eines Trojaners kein Problem dargestellt, denn schließlich hatte es ja auch mehrere Viren und in einem Falle ein »normaler« Trojaner auf die Festplatte geschafft, wobei nicht geklärt werden konnte, ob und welche Daten den Rechner verlassen hatten: Die Firewallwarnmeldungen wurden irgendwann ignoriert und in jedem Fall wäre ihr Schutz nach der Infektion des Computers mit der Schadsoftware nur noch relativ gewesen, da solche Programme in der Lage sind, bestimmte ProgrammRoutinen auszuführen, die Schutzmaßnahmen auf einfache Weise umgehen können. Man kann im Nachhinein nur die stille Hoffnung haben, dass keine 
sensiblen Daten mittels eines Keyloggers mitgeschnitten wurden, denn sonst wären Passwörter aller Art obsolet geworden. Im Übrigen sind die Passwörter selbst ähnlich problematisch: Keiner der beobachteten Computerbenützer fand Zeit für ein wirklich sicheres Passwort aus Zahlen und Buchstaben. Stattdessen wurde immer das gleiche benutzt und als Bonus sogar das Passwort für das Online-Banking bereitwillig preisgegeben. Der schlampige und unverantwortliche Umgang mit den entsprechenden Gefahren ist ein Phänomen, das in vielen Bereichen der IT-Welt auftritt. Trotz vielfältiger Warnungen, bestimmte unbekannte Emailanhänge unbedingt nicht zu öffnen, da ansonsten ein Computerwurm installiert würde, wurde weltweit genau dieser Ablauf tausendfach durchgeführt (vgl. SCHNEIER 2001: 255). Die Schadsoftware verschickte sich im Anschluss daran eigenständig an alle Emailadressen, die dem Mailprogramm bekannt waren und breitete sich nahezu unbegrenzt aus. Der entscheidende Punkt des Kontrollverlustes und damit auch der Verlust der Freiheit ist der Moment, in dem die Privatsphäre korrumpiert ist. Es gibt nun keine Möglichkeit, sich vollkommen zu schützen. Passwörter sind sinnlos, weil sie mitgeschnitten und übermittelt werden können, kryptographische Techniken sind wirkungslos: Jede Anzeige an einem Computerbildschirm kann manipuliert sein, denn man sieht nämlich nicht den Vorgang der im Computer direkt abläuft, sondern nur eine Anzeige auf dem Bildschirm, die den entsprechenden Vorgang dokumentiert (ebd.: 258). Es ist durchaus möglich, dass tatsächlich ein völlig anderer Prozess abläuft: Statt eine Datei zu verschlüsseln, was der Bildschirm anzeigt, wird die Datei in Wahrheit verschlüsselt und gleichzeitig als Kopie an einen anderen Adressaten verschickt, der dann vollen Zugriff auf den gesamten Inhalt hat. Die Konsequenz aus diesen Aspekten ist die folgende: Das Sichern der Privatsphäre ist aufwändig und erfordert Diszip- 
lin und Einschränkungen. Wissen um mögliche Probleme ist die unabding-bare Voraussetzung, um den Schutz auch tatsächlich realisieren zu können. Das wichtigste Prinzip ist die Kontinuität des Schutzes, denn ist ein Eingriff von außen erst einmal geschehen, gibt es kein gerechtfertigtes Vertrauen mehr in die eigene Privatsphäre. Jedes Datum, das der Einzelne von sich freigibt und veröffentlicht, ist damit der Eigenkontrolle entzogen:

»Nehmen Sie nur die Ungehemmtheit, mit der die Menschen neue Technologien wie das Internet nutzen und alles Mögliche von sich dabei gerne preisgeben. Seit es das Handy gibt, erfahre ich im Warteraum der Flughäfen lauter private Dinge meiner Mitreisenden, die sie besser bei sich behielten und von denen ich lieber verschont bliebe. Die modernen technologischen Entwicklungen erzwingen eigentlich eine neue Achtsamkeit: Wir müssen uns mühen, uns nicht ständig preiszugeben« ( $\mathrm{SCHÄUBLE} \mathrm{2008,} \mathrm{zitiert} \mathrm{nach}$ SCHMID 2008).

Dieser Aussage von Wolfgang SCHÄUBLE in einem Interview ist hinzuzufügen, dass es auch wichtig ist, zu berücksichtigen, dass Daten in der Regel längere Zeiträume überdauern und gespeichert werden. Was für Individuen gilt, betrifft auch größere Konfigurationen wie beispielsweise Firmen, die zusätzlich eventuell noch mit kriminellem Verhalten von Mitarbeitern klarkommen müssen, die auch erheblichen materiellen Schaden verursachen können.

Der Schritt aus dem privaten Kernbereich heraus birgt weitere Gefahren für die Freiheit. Hier ist es den Beteiligten nur sehr schwer möglich, sich den Überwachungsmechanismen zu entziehen. Dies resultiert auch aus der Tatsache, dass eine unglaublich große Zahl an Überwachenden tätig ist, die alle bestimmte, aber unterschiedliche Ziele verfolgen. Der Staat ist dabei ein big player, aber auch ein global player und er ist nicht alleine tätig. Ob Schengener Informationssysteme, Europol, Fluggastdatenabgleiche, Lauschangriffe, der Zugriff auf Telekommunikationsdaten, das Registrieren von DNS-Daten, 
der Zugriff auf Mautdaten und Vieles mehr stößt auf das Interesse von Datensammlern. Dazu kommen »zivile» Daten wie Suchmaschinenstatistiken, Einkäufe mit Kreditkarten und unter Benützung von Rabattsystemen, SCHUFAEinträge, Angaben bei Versicherungen, soziale Netzwerke des Web 2.0 (für eine Übersicht LACY 2008: 17 ff), Krankenakten usw. Der Abgleich der verschiedenen Daten birgt das größte Gefährdungspotenzial, da aus den enormen Datenmengen für jeden Einzelnen Profile angelegt werden können, welche die Bewegungen des Betreffenden sowohl im sozialen als auch im tatsächlichen Raum klar identifizieren. Somit gehen in letzter Konsequenz sowohl die Datensicherheit als auch die individuelle Freiheit verloren.

\subsubsection{Sicherheit durch Fremdkontrolle?}

Im öffentlichen Raum setzt sich die Überwachung fort, von Anonymität kann man daher nur bedingt sprechen, obwohl vermutlich ein Großteil der Bevölkerung sich dieser Tatsache nur bedingt bewusst ist. Überwachungsmaßnahmen an Tankstellen, in Geschäften, vor Geschäften, in Parkhäusern, in Kinos und an vielen anderen Orten werden weitgehend akzeptiert. Parallel dazu wird der öffentliche Raum nicht nur überwacht und kontrolliert, sondern gleichzeitig durch die in ihm handelnden Akteure in zunehmendem Maße privatisiert. Halbprivate Räume entstehen in Einkaufspassagen und Einkaufszentren, Bahnhöfen und U-Bahnen und Krankenhäusern, in denen zunehmend private Sicherheitsdienstleister als Ergänzung zu staatlichen Behörden tätig werden, während der Staat als traditioneller Sicherheitsproduzent mit Personalkosten zu kämpfen hat. Gleichzeitig wird der öffentliche Raum zur großen Schaubühne: Wir dürfen Handygespräche der anderen mitanhören und 
können mit denselben Geräten auch gleich Fotos schießen und bei Bedarf ein Video drehen, für das es eventuell sogar einen Markt gibt (bild.de 2008). Die Banalität dieser Videos - »Macht diese Eisbärbraut Knut glücklich?« »Geile Schildkröte missbraucht Schuh« »Video-Künstler Klaus Schweitzer hat seine Terrier Mausi und Mopsi gefilmt (...)« (ebd.) - ist eigenartig aber leider nicht einzigartig. In der Öffentlichkeit des World Wide Webs sehen wir auf der Webseite youtube.de private Videos und auf youporn.com noch privatere Videos. Wir treffen auf Big Brother, nur diesmal nicht als Überwachungsleviathan, sondern in Gestalt einer Fernseh-Containershow, wo im Grunde alles, nur nicht die Bilder von Toilettengängen, im Fernsehen oder Internet gezeigt (vgl. Mıкos u.a. 2000: 31) wird. Auf diese Weise tritt neben die Banalität die Neugier, die hier alle Wesensmerkmale des Voyeurismus aufweist. Letztlich können wir im Internet über die einschlägigen Portale nicht nur neue Partner fürs Leben finden, sondern wir können am Ende dort auch professionell trauern (für eine Übersicht, vgl. CASSALA 2008) oder sogar jungen Menschen beim Selbstmord zusehen (vgl. PATALON 2008). Durch diese umfassende Veröffentlichung des Privaten wird die Öffentlichkeit zugleich privat angeeignet. Das Gefühl für die Wahrnehmung sozialer Distanzen geht in gleichem Maße verloren.

Die organisierte Kontrolle des privaten Raumes, um Sicherheit zu gewährleisten, fällt sehr schwer. Dies liegt zum einen daran, dass die zur Auswertung zur Verfügung stehenden Daten immer noch sehr unverbunden und verstreut zur Verfügung stehen und dass der letzte Schritt hin zur völligen Überwachungsgesellschaft noch nicht getan wurde. Denn trotz aller viel diskutierten Maßnahmen etablieren sich neue Techniken noch nicht als Massenphänomen. Noch werden unsere Kinder nicht mit einem GPS-Gerät ausgestattet, das 
automatisch Alarm schlägt, wenn sie bestimmte Gegenden betreten - obwohl es diese Möglichkeit heute sehr wohl gibt (jackmobile.de 2008) - und noch erkennen uns Überwachungskameras nicht automatisch und gleichen unsere biometrischen Daten mit registrierten Profilen ab. Unsere Autos werden noch nicht automatisch wegen unbezahlter Strafzettel stillgelegt, wenn wir ein Verkehrsleitsystem durchfahren. Noch immer sind viele benutzte Datenbanksysteme unverbunden und nicht kompatibel, auch weil die Hersteller dieser Programme ihren Code lieber unter Verschluss halten - die Überwachung mit Hilfe von Open-Source-Software wäre ein Paradoxon. Stattdessen wird versucht, mit den zur Verfügung stehenden Mitteln in unserer Gesellschaft, Sicherheit zu produzieren. Doch gelingt dies durch die Kontrollmechanismen auch?

\subsubsection{Sicherheitsversprechen - statt Freiheit}

Die klassische Gegenüberstellung von Sicherheit, die auf Kosten der Freiheit ausgeweitet ist, bildet die Realität nicht exakt ab. Man gewinnt auf den ersten Blick den Eindruck, dass beide unmittelbar miteinander verknüpft wären. In der Realität stimmt dieses Bild aber nur bedingt. Eingriffe in die Freiheit werden in der Regel durch die politische Argumentation als Beitrag zur Steigerung der Sicherheit verkauft. In bestimmten Fällen, etwa wenn konkrete Straftaten durch einen Eingriff in die Freiheit Einzelner aufgedeckt werden können, ist die Einschätzung auf den ersten Blick korrekt.

Die unkontrollierte Anwendung von freiheitseinschränkenden Maßnahmen aber, die ohne Verdacht und anlassunabhängig ermöglicht werden, zielen nicht auf die Aufklärung konkreter Tatsachen, sondern ins »kriminelle Blau«. Dieser 
Vorgang markiert den Übergang von der Sicherheit hin zur Prävention. Denn hier liegt das Interesse nun nicht mehr nur darin, Täter bloß zu ergreifen, sondern schon im Vorfeld der Planung der möglichen Tat zur Abwehr von Gefahren einzugreifen. Der Sicherheitsstaat begibt sich aber damit auf ein Tätigkeitsfeld, in dem er nur sehr mühsam effektiv wirksam sein kann, denn den Anspruch der Prävention kann er nur punktuell einlösen. Stattdessen entsteht ständig neuer Handlungsbedarf - jedes begangene schwerwiegende Verbrechen und jedes Risiko wird so zu einer Aufforderung zu mehr sicherheitsrelevantem Handeln und die Ansprüche steigen kontinuierlich weiter. Egal ob Rasterfahndung, Lauschangriff, Videoüberwachung, Telefonüber-wachung, Online-Überwachung, Vorratsdatenspeicherung und Bewegungsprofile, Gendatenbanken, Datenbanken oder Informanten eingesetzt werden, immer bestehen für Normalbürger und natürlich auch für Kriminelle Möglichkeiten, den Zugriffen und Kontrollmechanismen zu entgehen. Dazu steht ein umfangreiches Arsenal an technischen Möglichkeiten zu Verfügung, das gleichzeitig noch die Möglichkeit bietet, den Verdacht gezielt auf andere abzuwälzen. Dabei muss man gar nicht an besonders komplexe Hightech-Lösungen denken, wie etwa an das Verbergen einer verschlüsselten Datei in einem digitalen Bild (vgl. GAIJIN 2008), das dann über eine verschlüsselte Onlinefestplatte wie wuala (vgl. wuala.de 2008) verbreitet wird oder dem Benützen eines Computers, der mittels eines Bot-Netzwerks gekapert wurde. Alternativ sind auch diverse Lowtechlösungen denkbar, wie ein Treffen an einem übersichtlichen und nicht überwachten Ort oder das gezielte Ausbringen von falschen Genspuren an Tatorten. Es kommt in diesem Zusammenhang vielleicht einmal die Zeit, in der wir unseren Müll und die geschnittenen Haare nach einem Friseurbesuch gezielt vernichten werden, um keinen Raum für Manipulationen 
zu lassen. Zusammengefasst kann man sagen, dass die Prävention nur tendenziell gelingen kann, da multiple Faktoren und zu viele Handlungsalternativen im Wege stehen. Das Reduzieren der Freiheit geht somit einher mit dem bloßen Versprechen, dass mehr Sicherheit entsteht und nicht mit mehr Sicherheit selbst.

Langfristige Prognosen fallen vielen wissenschaftlichen Disziplinen schwer; statt langfristiger Blicke in die Zukunft konzentriert man sich allzu oft auf theoretische Gebilde und Aussagen mittlerer Reichweite (zum Begriff, vgl. MERTON 1968). Dies ist auf Grund der Vielzahl an beteiligten Faktoren auch hier sinnvoll. Viel wird davon abhängen, wie sich das Bewusstsein der Beteiligten verändert und welche Inhalte und Vorstellungen mit den Begriffen Freiheit und Sicherheit verbunden werden. Trotzdem ist davon auszugehen, dass einmal begonnene Entwicklungen nur sehr selten komplett revidiert werden. Stattdessen wird Terrain für die Freiheit nur mit viel Mühe und Aufwand und einem »guten Draht nach Karlsruhe« zurück gewonnen.

Einfacher fällt es in diesem Zusammenhang, ganz neue Gebiete für die Freiheit zu erschließen, nämlich dadurch, dass die Freiheit in den Medien vermehrte Aufmerksamkeit erhält. Der Schutz der Privatsphäre ist zwar durchbrochen und es wird sehr schwer fallen, entsprechende Barrieren wieder zu errichten. Trotzdem sind eine Vielzahl von Aktiven für die Freiheit im Einsatz. Datenpannen werden aufgedeckt und die schlimmsten Überwacher bekommen durch das World Wide Web unliebsame Aufmerksamkeit wie beispielsweise durch den Big Brother Award, der seid 1998 an die schlimmsten Datenkraken verliehen wird (vgl. bigbrotherawards.de). Die »Gewinner« sind dabei prominente und unbekanntere Zeitgenossen, die als kleine und große Big Brother auffällig werden. Ein Blick in das Jahr 2005 zeigt die Vielfalt: 
»Lifetime. Otto Schily (..), ehemaliger Bundesminister des Inneren, erhält den Preis für die undemokratische Einführung des biometrischen Reisepasses, für sein ıLebenswerkı, nämlich für den Ausbau des deutschen und europäischen Überwachungssystems auf Kosten der Bürger- und Freiheitsrechte und für seine hartnäckigen Bemühungen um die Aushöhlung des Datenschutzes unter dem Deckmantel von Sicherheit und Terrorbekämpfung.

Wirtschaft. Saatgut-Treuhandverwaltungs-GmbH, (..) vertreten durch Geschäftsführer Dirk Otten, erhält den Preis für Datensammlung über Bauern; Verklagen von mehreren tausend auskunftsunwilligen Landwirten; Beschaffung der Kundendaten von Genossenschaften und verdeckte Testeinkäufe bei Bauern.

Behörden \& Verwaltung. Landesregierung Niedersachsen, die Regierung des Landes Niedersachsen, vertreten durch den Ministerpräsidenten Herrn Christian Wulff, erhält den Preis für die Zerschlagung der Datenschutzaufsicht in Niedersachsen.

Kommunikation. (...) die Generalstaatsanwaltschaft des Landes Schleswig-Holstein, vertreten durch Erhard Rex, erhält den Preis für die großflächige Fahndung nach Zeugen (die wie Verdächtige behandelt wurden) mittels Handy-Ortung.

Technik. Videoüberwachung (Richter, zahlreiche Organisationen) Videoüberwachung allerorten - diverse Kandidaten erhalten den Preis gemeinsam für die schleichende Degradierung von Menschen zu überwachten Objekten und der Verharmlosung der Folgen von flächendeckender Überwachung: z.B. Richter, ÖPNV-Betreiber, die Deutsche Bahn, Polizeibehörden, Buchhändler, der Macintosh-Großhändler GRAVIS...

Verbraucherschutz. WM-Organisationskomittee des DFB (..), vertreten durch Franz Beckenbauer, erhält den Preis für die inquisitorischen Fragenbögen zur Bestellung von WM-Tickets, für die geplante Weitergabe der Adressen an die FIFA und deren Sponsoren und für die Nutzung von RFID-Schnüffelchips in den WM-Tickets.

Politik. (..) Volker Bouffier, der Hessische Minister des Inneren, Volker Bouffier, erhält den Preis für das spräventive` Orten und Abhören von Mobiltelefonen; für die DNA-Analyse bei Kindern unter 14 Jahren, die eine Straftat begangen haben zu deren zukünftiger Strafverfolgung; für die Befugnis der hessischen Polizei, Kfz-Kennzeichen auch ohne Straftatverdacht zu scannen und für den Einsatz von Videoüberwachung bei Personenkontrollen.

Regionalpreis. (..) Die Grundschule Ennigloh bei Bünde teilt sich den Preis mit der Volksbank Bad Oeynhausen Herford eG und der Sparkasse Herford für die Weitergabe der 
Namen von Schulanfängern an die genannten Geldinstitute zum Zwecke der Werbung (`Startkonto८) ohne Einwilligung der Eltern.« (ebd.)

Auffällig sind bei dieser mehr oder weniger illustren Runde an »ausgezeichneten« Personen und Einrichtungen die Vielfalt und die vielen Bereiche, in denen Eingriffe in die Privatsphäre und ungezügelte Datensammelwut stattfinden. Es sind keinesfalls immer nur staatliche Stellen, die hemmungslos Daten sammeln und zur Strafverfolgung einsetzen, sondern es sind gerade auch zivile Einrichtungen, die ungeniert Daten sammeln, auch an unerwarteten Stellen, wie das Beispiel der Saatgut-Treuhand Verwaltungs GmbH (Bereich Wirtschaft) zeigt. In der ausführlichen Begründung der Award-Verleihung ist unter anderem davon die Rede, dass die Firma Lizenzverstöße dadurch aufzudecken versucht, indem sie den »gläsernen Landwirt« (ebd.) kreiert, dessen Daten wie Landflächen, Anbauzyklen, Düngung und Anbauquantitäten zusätzlich zur gezielten Bewerbung durch die chemische Industrie genützt werden. In letzter Konsequenz beeinflusst dies die Agrarmärkte durch die gesteigerten Profitchancen für bestimmte Gruppen - auch andere wie beispielsweise die Konsumenten. Auch andere Beispiele aus oben stehender Liste machen deutlich, dass die angesprochenen Probleme nicht isoliert zu sehen sind, sondern meistens einen größeren Bereich von Betroffenen angehen oder sich auch vordergründig durch eine augenscheinliche Harmlosigkeit charkterisieren lassen. Dazu zählen die Beispiele der Sparkasse Herford, die sich aus Gründen der gezielten Werbung die Daten von eingeschulten Kindern besorgen konnten oder auch der Einsatz der RFID-Technologie in den Eintrittskarten der Fußball-WM. Besonders im letzteren Fall kann der Eindruck entstehen, man sei nicht unmittelbar betroffen, indem man sich schlichtweg keine Eintrittkarte kauft und damit auch nicht die verschiedenen Daten über sich 
preisgeben muss. Allerdings besteht immer auch die Gefahr, dass mit solch begrenzten Projekten gewissermaßen Feldforschung betrieben wird und dass entsprechende Gepflogenheiten und Techniken sich schleichend ausweiten und etablieren.

Kann man noch von Überwachung sprechen, wenn man weiß, wer überwacht? Die Vielfalt der Preisträger setzt sich über die Jahre hinweg fort, wobei die Botschaft der Webseite natürlich nicht einer gewissen Polemik entbehrt WOLFGANG SCHÄUBLE beispielsweise wird im Jahr 2007 ausdrücklich für dieses Jahr als Nicht-Preisträger ausgezeichnet, nachdem er in diversen Jahren zuvor regelmäßig bedacht worden war.

Eingriffe in verschiedene Grundrechte werden auch durch die Printmedien, etwa durch die Reihe »Grundrechte-Report« (vgl. z.B. zur Passagierdatenübermittlung GAYK/SOKOL (2004: 32 ff.) kritisch dokumentiert und bleiben in der öffentlichen Diskussion. Sowohl Sicherheit als auch Freiheit benötigen die offene Auseinandersetzung und auch den politischen Streit um das Thema, zumindest, wenn man versucht, ihr Verhältnis und ihre Entwicklung weiter zu dokumentieren. Der freiheitliche demokratische Verfassungsstaat ist dabei die wichtigste Konstante. Die wichtigste Erkenntnis dabei ist, dass der Staat in diesem Staat sowohl als Akteur und auch als Betroffener auftritt. Er grenzt die Freiheit ein durch Überwachungsmaßnahmen und kann selbst Opfer von Überwachung werden. Gleiches gilt auch für alle Arten von Gruppierungen und auch für den Einzelnen. Es ist ebenfalls möglich, dass sich Freiheit und Sicherheit, fasst man die Begriffe wieder im Sinne ihrer möglichen Bedeutungen weiter, auch unabhängig voneinander verändern. Dies gilt vor allem dann, wenn die existierende Sicherheitspolitik innerhalb der schon abgesteckten Grenzen optimiert wird, ohne weitere Freiheitsrechte einzuschränken. Eine 
Möglichkeit in diesem Sinne wäre, die Polizeien der Bundesländer technisch, personell und letztlich finanziell aufzurüsten und die Kooperation der Behörden weiter zu verbessern. Im Bereich der Telekommunikationsüberwachung würde es bereits eine Verbesserung darstellen, wenn die vorhandenen Kontrollmöglichkeiten wie der Richtervorbehalt ausgeschöpft würden und eine gewisse Transparenz gewährleistet würde.

Trotzdem ist letztlich davon auszugehen, dass die Ausrichtung auf Sicherheit und Prävention stabil bleiben wird. HASSEMER fordert deshalb, aktiv und offensiv für die Verwirklichung der Grundrechte in einer solchermaßen ausgerichteten Gesellschaft zu werben (vgl. HASSEMER 2002: 5). Er weist an gleicher Stelle allerdings auch darauf hin, dass es nicht genüge, bestimmte Sicherheitsinitiativen pauschal als grundrechtsbeeinträchtigend anzuprangern und fordert stattdessen, grundrechtsfreundliche Alternativen zu präsentieren. Eine derart kontruktive Haltung in diesen sensiblen Fragen wäre aber in letzter Konsequenz nicht nur den Sicherheitskritikern anzuraten, sondern prinzipiell allen Beteiligten, da die Grundrechtsfreundlichkeit die zentrale Bedingung für entsprechende gesetzliche Veränderungen darstellt. Veränderungen, die diesem Geiste entsprechend stattfinden, geben dann auch eine Antwort auf die Frage »Sicherheit statt Freiheit« und sie lautet: Freiheit und Sicherheit und zwar sowohl als auch. 


\section{Schlussbetrachtung}

Sicherheit und Freiheit stehen in einem besonderen Verhältnis zueinander und es hat sich im Fortgang der Untersuchung gezeigt, dass dieses sich am besten veranschaulichen lässt, wenn man drei Bereiche unterscheidet (vgl. Abbildung 7, S. *noch unbekannt*). Sowohl Sicherheit als auch Freiheit sind Phänomene, die man einerseits isoliert betrachten kann und die andererseits auch in enger Verbindung zueinander stehen. Die Entwicklung von Sicherheit alleine ist dabei in der Rückschau bemerkenswert. Sie wurde zum zentralen Motiv bei einer Fülle an Maßnahmen; dazu zählen die TKÜ, die akustische Wohnraumüberwachung, Handyortung, Videoüberwachung öffentlicher Plätze, Rasterfahndung, Schleierfahndung und vieles mehr. Vor allem im Bereich der Inneren Sicherheit, soll mit diesen verschiedenen Formen der Fremdkontrolle Sicherheit garantiert werden. Zugleich verschiebt sich gewissermaßen die Zeitlinie der ausgeübten Kontrolle. Es genügt nicht mehr, mit Hilfe der verschiedenen Maßnahmen Kontrolle auszuüben und Straftaten zu verfolgen, sondern im Mittelpunkt steht nun die Prävention, Gefahrenabwehr und Risikominimierung schon im Vorfeld der möglichen Tat. Die Videoüberwachung öffentlicher Plätze ist hier ein sehr plastisches Beispiel: Das Auge der Kamera durchdringt den öffentlichen Raum und signalisiert eine kontinuierliche Sanktionsmöglichkeit und Sanktionsbereitschaft. Es ist nun an dem Einzelnen, sein Verhalten auf diese Überwachung abzustimmen und Konformität vor dem Auge der Kamera zu demonstrieren.

Die öffentliche Zustimmung zu diesen Maßnahmen steigt und sinkt mit dem subjektiven Sicherheitsgefühl, das oftmals nach bestimmten Straftaten erheblich absinkt, obwohl sich die objektive Bedrohungslage nur unmerklich ver- 
ändert. Es fällt bemerkenswert schwer einzuschätzen, ob die vielen Sicherheitsmaßnahmen auch objektiv die Sicherheitslage verbessern. Dies liegt vor allem daran, dass das umfassende Präventionsziel dafür sorgt, dass die Zielerreichung zunehmend unscharf wird. Auffallend ist aber die Tatsache, dass vor allem bei Sicherheitspaketen und anderen bedeutsamen rechtlichen Veränderungen - wie schon gezeigt - die Terrorgefahr und Organisierte Kriminalität als Begründung angeführt werden, obwohl eine Vielzahl der Maßnahmen eher breit streut und eher »Normverletzungen geringen Unrechtsgehalts« (Strasser/SchWEer: 177) ans Tageslicht kommen. Die zentrale Frage, ob so Sicherheit tatsächlich erhöht wird oder ob sich die Unsicherheitsfaktoren nur rein räumlich verschieben, ist hier nicht zu beantworten, obwohl man als Bürger nur hoffen kann, dass man eben nicht Opfer von Organisierter Kriminalität oder Terrorismus wird.

Betrachtet man die Entwicklung der Freiheit für sich genommen, so ergibt sich ein zweigeteiltes Bild: Gerade die europäischen Einigungsprozesse haben abseits aller kritischen Betrachtung die wirtschaftliche und politische Freiheit ihrer Bürger deutlich erhöht. An dieser Stelle gibt es sozusagen keinen direkten Zusammenhang mit Sicherheit. Bei einer Vielzahl von Phänomenen entstehen aber mannigfaltige Berührungspunkte zwischen Sicherheit und Freiheit. Viele der in Kapitel 3 und 4 beschriebenen rechtlichen Veränderungen, lassen in ihren Auswirkungen die Sicherheitsbestrebungen mit der individuellen Freiheit kollidieren. Dieser Konflikt lässt sich mit dem Kampf um die Kontrolle der Privatheit am besten beschreiben, da praktisch alle Sicherheitsmaßnahmen natürlich graduell abgestuft - die Privatheit berühren und einschränken. Der vom Bundesverfassungsgericht festgestellte Kernbereich privater Lebensführung und das Grundrecht auf informationelle Selbstbestimmung (vgl. Kapitel 
130) sind tatsächlich in Dauergefährdung: Hier engt das Streben nach Sicherheit tatsächlich die Freiheit des Einzelnen ein.

Ein wichtiger Schlüsselaspekt dieses Zusammenspiels von Sicherheit und Freiheit ist der technische Fortschritt, der das gesamte Gefährungspotenzial repräsentiert. Technik beeinflusst die Prävention, indem sie neue Herausforderungen produziert und gleichzeitig neue Präventionsmethoden generiert. Sie ermöglicht erst das Funktionieren von Kommunikationwegen auf denen der Einzelne unter Fremdkontrolle gerät und über die er versuchen kann, sich vor Fremdbestimmung zu schützen. Das Ringen nach Sicherheit wird in diesem Sinne gekennzeichnet durch den Versuch, im Bereich der technischen Entwicklung schrittzuhalten und die so entstehenden Instrumente für das Sicherheitsstreben auszubauen

Im Konflikt zwischen Sicherheit und Freiheit nimmt mittlerweile das Bundesverfassungsgericht eine wichtige politische Rolle ein. Es wird in schöner Regelmäßigkeit bemüht und beeinflusst dann mit seinen Entscheidungen die politischen Abläufe entscheidend - man denke nur an die weitreichenden Konsequenzen des Volkszählungsurteils. Der so entstehende politische Kurs pendelt analog zur Entwicklung von Sicherheit und Freiheit hin und her. Freiheitseinschränkende gesetzliche Regelungen werden etabliert und dann vom Bundesverfassungsgericht »kassiert« - ein aktuelles Beispiel ist die vorsorglich anlasslose Vorratsdatenspeicherung (vgl. BvR 256/o8), die unter anderem auch deswegen gekippt wurde weil »sie ein Gefühl des ständigen Überwachtwerdens« und der »diffusen Bedrohlichkeit« (ebd.: Abs. 240 ff) auslösen kann. Insgesamt betrachtet kann man sich dem Eindruck nicht entziehen, dass es in diesem Hin und Her für die Freiheit immer wieder zwei Schritte zurück und dann wieder einen Schritt vorgeht und dass sich das Verhältnis kontinuierlich 
hin zu vermeintlich mehr Sicherheit bewegt. Wie in in Kapitel 4 versucht wurde zu zeigen, ist es mehr als fraglich, ob durch die Veränderungen auch tatsächlich mehr Sicherheit im objektiven Sinne geschaffen wird oder ob es sich dabei insgesamt nur um symbolische Politik handelt. Der Prozess der Veränderungen von Sicherheit und Freiheit ist nicht abgeschlossen und kann es fast zwangsläufig auch nicht sein. Beide Begriffe sind kulturell überformt und wandeln sich im Laufe der Zeit. Oftmals sind Veränderungen in diesem Prozess nur graduell, manchmal ereignen sich drastische Zäsuren. Bei manchen dieser Einschnitte, wie beispielsweise bei den Notstandsgesetzen, sind die tatsächlichen Auswirkungen minimal oder wirken nur latent: „Trotz kritischer Alarmrufe sind die Demokratien der westlichen Hemisphäre weit entfernt vom drakonischen Regime eines Ausnahmezustandes. Der Notstand ist nicht das herrschende Paradigma« (SOFSkY 2005: 153). Trotzdem werden in der politischen Diskussion immer wieder Themen mit großer Emotionalität benützt, um Maßnahmen zugunsten der Sicherheit und zu Lasten der Freiheit zu rechtfertigen. Dazu zählen unter anderem Themen wie Terrorismus, Kindesmissbrauch oder Mord. In diesen Fällen setzt sich öfter die Ansicht durch, der Zweck heilige alle Mittel, was auch vom Grad der persönlichen Betroffenheit abhängt. Auf keinen Fall darf vergessen werden, dass mitunter die Betroffenheit und die entsprechende Argumentation auch durch andere Aspekte wie politische Taktik, die besondere Funktion eines Amtes (z.B.: Innenminister versus Bundesdatenschutzbeauftragter), wirtschaftliche Interessen, persönliche Motive oder die zeitliche Distanz zur nächsten Wahl eine wichtige Rolle spielen.

Der Vergleich mit anderen Gesellschaften mit unterschiedlicher gesellschaftlicher und vor allem auch politischer Struktur wirkt ebenfalls mit auf den 
Änderungsprozess ein: Es ist leichter, sich als frei einzuschätzen, wenn der Vergleichsmaßstab die »Diktatur von nebenan« ist.

Sehr leicht geraten die verschiedenen Aspekte von Freiheit außerdem ins Hintertreffen, wenn die Rahmenbedingungen nicht stimmen und wirtschaftliche und materielle Probleme an Relevanz gewinnen. Dies zeigen auf ihre Weise auch die aktuellen Ereignisse am weltweiten Aktienmarkt: Wie nach vielen Jahrhundertereignissen wie etwa den Wertverlusten an den Börsen im Jahre 2008, wird der Ruf nach staatlichen Kontrollmechanismen laut. Von unternehmerischer Freiheit ist in diesen Tagen eher wenig zu hören. Wieder hergestellte Sicherheit im wirtschaftlichen Bereich verdrängt sehr schnell den Wunsch nach Freiheit. Die Konsequenzen für die Freiheit sind in so einem Falle sehr gravierend. Sie mutiert zu einer Schönwetterfreiheit, die nur dann eine Chance bekommt, wenn sie gerade bezahlbar ist. Sicherheit selbst wird im Gegenzug immer mehr zu einem geschätzten Gut, das in der Lage ist, in kleineren Marktwirtschaften zu einem bestimmenden Industriezweig zu werden (vgl. KLEIN 2007: 435, sie bezieht sich dabei auf Israel). Wie bei jedem Gut ist es unvermeidlich, dass früher oder später dafür zu bezahlen ist, wobei wie meistens ein Großteil der Kosten auf den Endverbraucher abgewälzt wird. Es bleibt nur zu hoffen, dass langfristig diese Sicherheit mit Geld zu bezahlen bleibt und nicht routinemäßig auf die Währung Freiheit umgestellt wird.

Viel wird davon abhängen, wie sich die Einstellungen der Bürger zu Sicherheit und Freiheit auf breiter Front verändern und die entsprechenden Experten nicht mehr nur als einsame Rufer in der (Sicherheits-)Wüste fungieren. Eine Entwicklung hin zu mehr Freiheit kann nur dann einsetzen, wenn das Thema bewusst wird und zu entsprechendem politischen Handeln der Bürger führt die Gefahr dabei ist, dass die Thematik von tagespolitischen Inhalten (Arbeits- 
marktzahlen, Bankenkrise, etc.) immer wieder in den Hintergrund gerät. Dies ist letztlich auch der Grund, warum die Einrichtungen (BVerfG) und Individuuen, die sich bisher mit der Thematik beschäftigt haben, weiterhin öffentlichwirksam tätig bleiben.

Zum Abschluss dieser Arbeit bleibt noch festzustellen, dass die aktuellen Ereignisse während des Bearbeitungszeitraums oftmals erforderten, neue Entwicklungen mit einzubeziehen, um Aktualität zu gewährleisten. Die Entwicklungen bleiben nicht stehen, sie orientieren sich wie diese Arbeit öfter an vergangenen Ereignissen. Am 15.12.2008, dem Stichtag für diese Arbeit wurde die folgende Schlagzeile als letzte relevante zum Thema Freiheit und Sicherheit registriert:

»25 Jahre Volkszählungsurteil - Super-GAU des Datenschutzes - Der Präsident des Bundesverfassungsgerichts, Hans-Jürgen Papier, hat eindringlich vor einer Überwachungsgesellschaft internationalen Ausmaßes gewarnt. Seine Sorge gilt allerdings nicht den staatlichen Eingriffen... Angesichts fast wöchentlich auftretender Datendiebstahlsskandale müsse der Staat für ein angemessenes Schutzregime sorgen, forderte Papier zum 25. Jahrestag des Volkszählungsurteils bei einer Konferenz der Datenschutzbeauftragten in Karlsruhe. Der Gerichtspräsident rief den Gesetzgeber zwar dazu auf, bei der Bekämpfung und Verhinderung von Kriminalität die Gewichte von Freiheit und Sicherheit nicht grundlegend zu verschieben. Vor allem aber müsse sich der Staat um die Gefahren der von Privaten gespeicherten Informationen kümmern, die viele auch noch weitgehend freiwillig preisgeben würden.« (KERSCHER 2008)

PAPIER verweist hier auf wichtige Punkte. Es gibt im Gegensatz zur Situation zu Zeiten des Volkszählungsurteils keine klare Frontstellung mehr zwischen staatlichen Überwachern einerseits und Individuen als Überwachten andererseits. Zwar macht sie rein quantitativ immer noch einen Großteil der Problematik aus, allerdings hat sich das Gesamtbild ausdifferenziert. Die Gewichtung zwischen Sicherheit und Freiheit hat sich schon längst ver- 
schoben, allerdings sind alle Beteiligten Akteure an dieser Verschiebung beteiligt. Der Hunger nach Daten und Überwachung und damit nach mehr Sicherheit bestimmt das Handeln derjenigen, die sich davon einen Nutzen erhoffen. Gleichzeitig steigt die Bereitschaft vieler, sich zu exponieren und mehr von sich preiszugeben als unbedingt nötig wäre. Freiheit im Sinne von Privatheit wird damit stückchenweise aufgegeben und ist - wie versucht wurde zu zeigen - nur mit einigem Aufwand individuell zurück zu erlangen. Die Forderung PAPIERS an die Gesetzgebung, diesen Entwicklungen entgegen zu steuern und sich nicht nur gegen ein grundlegende Verschiebung von Freiheit und Sicherheit zu wenden, sondern sich ergänzend auch um die privaten Sammler und diejenigen kümmern, die bereitwillig Daten von sich preisgeben, ist richtig und wichtig. Allerdings stellt sich ebenfalls die Frage, ob die Gestaltungsfähigkeit und die Sensibilität der Legislative dazu ausreicht, diese Anforderungen zu bewältigen. Chancen dazu gab es und wird es auch künftig noch reichhaltig geben.

Es ist davon auszugehen, dass das Thema noch langfristig von öffentlichem und wissenschaftlichen Interesse sein wird und auch künftig nicht an Brisanz verlieren wird. 


\section{Literaturverzeichnis}

\section{Webseiten ohne Autorenangabe}

arcadia-potsdam.com (2008): Arcadia Potsdam,

http://www.arcadia-potsdam.com/, 21.12.2008, 11 Uhr;

bfdi.bund.de (2008): Schwerpunkte,

http://www.bfdi.bund.de/cln_027/nn_531940/DE/Schwerpunkte/Schwerpunkte_node.htm

I_nnn=true, 18.12.2008, $11 \mathrm{Uhr}$;

bigbrotherawards.de (2008): Willkommen bei den deutschen BigBrotherAwards, http://www.bigbrotherawards.de/, 12.12.2008, $21 \mathrm{Uhr}$;

bild.de (2008): Popstar Jimmy Kelly singt schon wieder in der Fußgängerzone, http://www.bild.de/BILD/news/leserreporter/home/leserreporter.html, 18.12.2008, 11 Uhr;

bka-petition.de (2008): Online-Petition gegen das BKA-Gesetz,

http://www.bka-petition.de, 12.12.2008, 11 Uhr;

bpb.de (2008): Experten-Forum: Anti-Terrorpolitik in Deutschland,

http://www.bpb.de/themen/ZL1B4U,o,o,Dokumente_und_Links.html, 12.12.2008, 10 Uhr;

bsi.de (2008): Computer-Viren, http://www.bsi.de/av/index.htm, 18.12.2008, 12 Uhr;

bundestag.de (2007 a): Tornado-Einsatz im Innenausschuss kritisiert, http://www.bundestag.de/aktuell/hib/2007/2007_169/02.html, 27.6.2007, 13.43 Uhr;

bundestag.de (2008a): Ständig aktualisierte Fassung der öffentlichen Liste über die Registrierung von Verbänden und deren Vertretern, Stand: 28.11.2008, http://www.bundestag.de/wissen/archiv/sachgeb/lobbyliste/lobbylisteaktuell.pdf, 1.12.2008, 17 Uhr;

daten-speicherung.de (2008): Kartentausch, http://www.daten-speicherung.de/index.php/kartentausch/, 12.12.2008, $17 \mathrm{Uhr}$; 
eff.org (2008): What is EFF?, http://www.eff.org/, 18.12.2008, $11 \mathrm{Uhr}$;

forums.somethingawful.com (2003):

http://forums.somethingawful.com/showthread.php?s=\&threadid=1107178\&perpage $=40 \& p$ agenumber=1, 19.11. 2003, $17 \mathrm{Uhr}$;

freenet.de (2008): Illegaler Datenhandel weitet sich aus, http://www.freenet.de/freenet/nachrichten/politik/200808/200808_4eb48ffdgd423efb813d da97aa5ddff8.html, 12.12. 2008, $11 \mathrm{Uhr}$;

gadgettrak.com (2008): MacTrak ${ }^{\mathrm{TM}}$ from GadgetTrak®: Flickr Integration Wi-Fi Positioning System with Flickr Integration, http://www.gadgettrak.com/products/mac/flickr, 12.12.2008, 12 Uhr;

gaijin.at (2008): SteganoG, http://www.gaijin.at/dlsteg.php, 12.12.2008, 11.34 Uhr;

jackmobile.de (2008): track your kid, http://www.jackmobile.de, 12.12. 2008, $13 \mathrm{Uhr}$;

ndr.de (2007): Heiligendamm: Sicherheitszaun fertiggestellt, http://www1.ndr.de/nachrichten/g8/zaun6.Html, 3.5.2007, 17.41 Uhr;

raceacrossamerica.org (2008):

http://www.raceacrossamerica.org/subwebraam/default.php?N_webcat_id=1, 25.9.2009, $10 \mathrm{Uhr}$;

securitas.de (2008): Securitas in Deutschland, http://www.securitas.com/de/ueber-securitas/securitas-deutschland/ 2.9.2008, 15.30 Uhr;

spiegel.de (2007): Randale in Rostock - 430 verletzte Polizisten, http://www.spiegel.de/politik/deutschland/o,1518,486280,00.Html, 2.7.2007, 10.16 Uhr;

sueddeutsche.de (2007 a): Die Festung ist verriegelt, G-8-Gipfel in Heiligendamm, 22.6.2007, 8.30 Uhr; www.sueddeutsche.de/deutschland/artikel/315/116199/article.Html,

suedeutsche.de (2007 b): Linke empören sich über bundesweite Razzia, www.sueddeutsche.de/deutschland/artikel/548/113435/article.html, 27.6. 2007, 9.20 Uhr; 
sueddeutsche.de (2007 c): Anrüchige Geruchsproben, www.sueddeutsche.de/deutschland/artikel/428/115313/article.html, 18.6.2007, 15.07 Uhr; suedeutsche.de (2007 d): Jung rechtfertigt Tornado-Tiefflüge, www.sueddeutsche.de/deutschland/artikel/550/119406/article.html, 20.6.2007, 10.02 Uhr; sueddeutsche.de (2007 e): Staatsschutz spricht von »punktuellen« Durchsuchungen, www.sueddeutsche.de/deutschland/artikel/813/115698/article.html; sueddeutsche.de (2008 a): BKA-Gesetz, Bund und Länder einigen sich auf Kompromiss, www. sueddeutsche.de/politik/394/450117/text/, 03.12.2008, 10:58 Uhr; wuala.de (2008): Wuala, der Online-Speicher, http://wuala.com/, 12.12. 2008, 11.34 Uhr; youporn.de (2008): www.youporn.de; 1.12.2007, $8 \mathrm{Uhr}$; youtube.de (2008); www.youtube.de; 1.12.2007, $8 \mathrm{Uhr}$;

\section{Quellen mit Autorenangabe}

Achsnich, G./Böhm, G./Dürig, M./Ebert, F./Roewer, H. (1997): Innere Sicherheit im vereinten Deutschland, Demokratie im Diskurs, Band 1, Erfurt;

Ackermann, C./Parsons, T. (1966): Der Begriff "Sozialsystem« als theoretisches Instrument, IN: PARSONS , T. (Hrsg.): Zur Theorie sozialer Systeme, herausgegeben und eingeleitet von Stefan Jensen, 69-84, Opladen;

ALBRECHT, H.-J. (2003): Rechtswirklichkeit und Effizienz der Überwachung der Telekommunikation nach den $\S \S 100 \mathrm{a}, 100 \mathrm{~b}$ StPO und anderer verdeckter Ermittlungsmaßnahmen, Forschung aktuell - research in brief Nr.17, Freiburg im Breisgau;

AlbRECHT, P.-A. (2003): Die vergessene Freiheit - Strafrechtsprinzipien in der europäischen Sicherheitsdebatte, IN: Friedrich-Ebert-Stiftung (Hrsg.): Sicherheit vor Freiheit? Terrorismusbekämpfung und die Sorge um den freiheitlichen Rechtsstaat, S. 9-19, Berlin;

ALBRECHT, U. (1992): Die Abwicklung der DDR, Die "2+4-Verhandlungen«, ein Insiderbericht, Opladen; 
ANHÄUSER, M. (2006): Der wahre Egoist kooperiert, http://www.sueddeutsche.de/wissen/artikel/788/89699/, 21.12.2006, 17.15 Uhr;

Appel, R./Hummel, D. (1988): Die Republik nach der Volkszählung, ein Beispiel für die Auseinandersetzung zwischen Herrschaftsapparat und außerparlamentarischer Bewegung, IN: Appel, R. (Hrsg.): Die neue Sicherheit: vom Notstand zur sozialen Kontrolle, S. 9-39, Köln;

BACHL, G. (1989): Lesen, Ein Weg in die Freiheit, Innsbruck, Wien;

BALL, K./Wood, D. (2006): Ein Bericht zur Überwachungsgesellschaft, Für den Datenschutzbeauftragten, Zusammenfassung, London;

Ballestrem, K. (1983): Vertragstheoretische Ansätze in der politischen Philosophie, Zeitschrift für Politik, Jahrgang 30, Heft 1, S. 1-17, München;

BANGE, D. (1992): Die dunkle Seite der Kindheit, Sexueller Mißbrauch an Mädchen und Jungen, Ausmaß - Hintergründe - Folgen, Köln.

Bange, D./Deegener, G. (1996): Sexueller Mißbrauch an Kindern, Ausmaß, Hintergründe, Folgen, Weinheim.

Bayerisches Staatsministerium der Finanzen, StFM (2006): Der bayerische Staatshaushalt, Nachtrag 2006, der erste Haushalt ohne Neuverschuldung, München;

BECK, U. (2007): Weltrisikogesellschaft, Frankfurt am Main;

BENDA, E. (1985): Politischer Gestaltungswille und Verfassungsauftrag, die friedenssichernde Aufgabe des Rechts, IN: RöPER, E. (Hrsg): Beiträge zur Inneren Sicherheit, S. 2-10, Kiel, Bremen;

Berger, P./Luckmann, T. (1980): Die gesellschaftliche Konstruktion der Wirklichkeit, eine Theorie der Wissenssoziologie, Frankfurt am Main;

BERLIN, I. (1995): Freiheit, Vier Versuche, Frankfurt am Main;

Bertelsmann Stiftung (Hrsg.) (1998): Kosten, Nutzen und Chancen der Osterweiterung für die Europäische Union, Gütersloh;

Beste, H. (2003): Zum Verhältnis von Polizei und profitorientierten Sicherheitsproduzenten, 
IN: LANGE, H.-J. (Hrsg.): Die Polizei der Gesellschaft, zur Soziologie der Inneren Sicherheit, S. 285-303, Opladen;

Beutelspacher, A. (2002): Kryptologie, eine Einführung in die Wissenschaft vom Verschlüsseln, Verbergen und Verheimlichen, ohne alle Geheimniskrämerei, aber nicht ohne hinterlistigen Schalk, dargestellt zum Nutzen und Ergötzen des allgemeinen Publikums, Braunschweig/Wiesbaden;

Beutels PaCher, A. u.a. (2005): Kryptographie in Theorie und Praxis, mathematische Grundlagen für elektronisches Geld, Internetsicherheit und Mobilfunk, Wiesbaden;

BIZER, J. (2003): Die Evaluierung der Telekommunikationsüberwachung, Kritische Anmerkungen zur MPI-Studie, IN: Kriminologisches Journal, 35. Jahrgang, Band 4, S. 280-295;

BMI (2007): Verfassungsschutzbericht 2007, Berlin;

Boecker, A. (2007): Deutschlands größter Polizeieinsatz, Ausnahmezustand in Heiligendamm,

http://www.sueddeutsche.de/deutschland/artikel/178/110068, 16.4. 2007, 10.39 Uhr;

Bolesch, C. (2008): Die Macht des Verdachts,

http://www.sueddeutsche.de/ausland/artikel/301/153905, 22.01.2008, 18 Uhr;

Bommarius, C (2008): Die Schattenseite von 1968, http://www.netzeitung.de/politik/deutschland/1213004.html

BORCHERS, D. (2008): Vor 25 Jahren: Informationelle Selbstbestimmung wird Grundrecht, http://www.heise.de/newsticker/Vor-25-Jahren-Informationelle-Selbstbestimmung-wirdGrundrecht--/meldung/120428, 15.12.2008, $12.05 \mathrm{Uhr}$;

Borchers, D./WILkens, A. (2007): Vor 20Jahren: 10 Minuten, die allen helfen (Update), http://www.heise.de/newsticker/Vor-20-Jahren-10-Minuten-die-allen-helfen-Update-meldung/90205, 10.12.2008, $11 \mathrm{Uhr}$;

Brandenburg, A. (1971): Systemzwang und Autonomie, Gesellschaft und Persönlichkeit bei TAtColt Parsons, Düsseldorf;

Breton, D. (1995): Lust am Risiko, Von Bungee-jumping, U-bahn-surfen und anderen Arten, das Schicksal herauszufordern, Frankfurt am Main; 
Breuer, R. (1999): Stabilität der Verfassung?, IN: ISEnsee, J./LeCheler, H. (Hrsg.): Freiheit und Eigentum, Festschrift für WALter LeISNER zum 70. Geburtstag, S. 301-339, Berlin;

BRIEGLEB, V. (2008): Kabinett beschließt Volkszählung für 2011, http://www.heise.de/newsticker/Kabinett-beschliesst-Volkszaehlung-fuer-2011-/meldung/119852, 03.12.2008, 22.32 Uhr;

Bukow, S. (2000): Deutschland: Mit Sicherheit weniger Freiheit über den Umweg Europa, IN: Glaessner, G.-J./LoRenZ, A. (Hrsg.): Europäisierung der inneren Sicherheit, eine vergleichende Untersuchung am Beispiel organisierter Kriminalität und Terrorismus, S.43-62, Wiesbaden;

Bundesministerium der Finanzen, BMF (2006): Haushaltsrechnung und Vermögensrechnung des Bundes für das Haushaltsjahr 2006 (Jahresrechnung 2006), Berlin;

BURDRICH, M. (Hrsg.) (2005): Die Erklärungen der RAF, Münster;

CASSALA, C. (2008): Immer mehr Anbieter im Netz bieten Raum für Trauer an, http://www.deutsche-startups.de/article_preview?id=3307, 13.10.2008, $8 \mathrm{Uhr}$;

CASSIRER, E. (1937): Determinismus und Indeterminismus in der modernen Physik, Göteborg;

Chambers, R. (1947): Thomas More, ein Staatsmann heinrich VIII, München, Kempten;

Clemens, T. (1995): Das BverfG im Rechts- und Verfassungsstaat, Mainz, München;

Cohen, S: (1993): Soziale Kontrolle und die Politik der Rekonstruktion, IN: Frehsee, D./Löschper, G./Schunmann, K. (Hrsg.): Strafrecht, soziale Kontrolle, soziale Disziplinierung, Jahrbuch für Rechtssoziologie und Rechtstheorie, Band XV, S. 209-238, Opladen;

DARWIN, C. (1998): The Origin of Species, Chatham;

DAVIDSON, J. (2007): Voice over IP Fundamentals, A systematic approach to understanding the basics od voice over IP, Indianapolis;

DAWKINS, R. (1978): Das egoistische Gen, Berlin;

Deleuze, G. (1993): Unterhandlungen, 1972-1990, Postskriptum über die Kontrollgesallschaften, Frankfurt am Main; 
Diekmann, A./Voss, T. (2004): Die Theorie rationalen Handelns, Stand und Perspektiven, IN: Diekmann, A./Voss, T. (Hrsg.): Rational-Choice-Theorie in den Sozialwissenschaften, Anwendungen und Probleme, München;

Dietl, W./Kai Hirschmann, K./Tophoven, R. (2006): Das Terrorismus-Lexikon: Täter, Opfer, Hintergründe, Frankfurt am Main;

Dillon, David (1994): Fortress America, In: Planning No.6, S. 8-12;

Dittmann, A. (2003): Grundsatz der Länderexekutive, IN: SACHS, M. (Hrsg.): Grundgesetz, Kommentar, München;

DoRsCH, C. (2005): Die Effizienz der Überwachung der Telekommunikation nach den §§ $100 \mathrm{a}$, 100 b StPO, Freiburg im Breisgau;

DRACH, M. (2006): Hirnforschung ist nur ein gutes Gefühl, www. sueddeutsche.de/wissen/artikel/113/74039/article.html, 26.4.2006, 12.18Uhr;

Dreher, E. (1987): Die Willensfreiheit, Ein zentrales Problem mit vielen Seiten, München;

Eisenberg, U./Puschke, J./Singelnstein, T. (2005): Ubiquitäres Computing = ubiquitäre Kontrolle?, IN: Kriminologisches Journal, 37. Jg, H 2;

ELIAS, N. (1969): Über den Prozess der Zivilisation, Soziogenetische und psychogenetische Untersuchungen, Erster Band, Bern, München;

Eschenburg, T. (1956): Staat und Gesellschaft in Deutschland, Stuttgart;

ESSEN, J. (2008): Telefonüberwachung,

http://www.joerg-van-essen.de/wcsite.php?wc_c=11636, 18.12.2008, 11 Uhr;

Euchner, W. (2001): John Locke (1632-1704), IN: MAIer, H./Denzer, H. (Hrsg.): Klassiker des politischen Denkens, zweiter Band, von John Locke bis MAX WeBER, S.15-31, München;

Feltes, T. (2009): Akteure der Inneren Sicherheit: Vom Öffentlichen zum Privaten, IN: LANGE, H.-J./ Ohly, P./REICherTZ, Jo. (Hrsg.): Auf der Suche nach neuer Sicherheit, Fakten, Theorien und Folgen, S. 105-113, Wiesbaden

Follath, E./SpörL, G. (2006): Der endlose Tag, IN: Der Spiegel, 36, 4. 9. 2006, S. 77-85; 
Foucault, M. (1976): Überwachen und Strafen, die Geburt des Gefängnisses, Frankfurt am Main;

Fraenkel, E. (1972): Der Pluralismus als Strukturelement der freiheitlich-rechtsstaatlichen Demokratie (1964), IN: Nuscheler, F./SteffanI, W. (Hrsg.): Pluralismus, Konzeptionen und Kontroversen, S.147-158, München;

Frevel, B. (2003): Polizei, Politik und Medien und der Umgang mit dem bürgerschaftlichen Sicherheitsgefühl, IN: LANGE, H.-J. (Hrsg): Die Polizei der Gesellschaft, zur Soziologie der Inneren Sicherheit, S. 321-337, Opladen;

FRIEDRICH, C. J. (1970): Politik als Prozeß der Gemeinschaftsbildung, eine empirische Theorie, Köln, Opladen;

Garfinkel, S: (2001): Database Nation: The Death of Privacy in the $21^{\text {st }}$ Century, Beijing u.a.;

GAYK, B./SOKOL, B. (2004): Was essen Terroristen? Passagierdatenübermittlung an die USZollbehörden, IN: MüLLER-Heidelberg U.a. (Hrsg.): Grundrechte-Report 2004, Zur Lage der Bürger- und Menschenrechte in Deutschland, S. 32-35, Frankfurt am Main;

Glassner, B. (2000): The Culture of Fear: Why Americans Are Afraid of the Wrong Things, New York;

Glaessner, G.-J./Lorenz, A. (2000): Europäisierung der Politik innerer Sicherheit - Konzept und Begrifflichkeiten, IN: GLAESSNER, G.-J./LORENZ, A. (Hrsg.): Europäisierung der inneren Sicherheit, eine vergleichende Untersuchung am Beispiel organisierter Kriminalität und Terrorismus, S. $7-17$, Wiesbaden;

Glaessner, G.-J. (2002): Sicherheit und Freiheit, IN: Aus Politik und Zeitgeschichte, B 10-11, S. 3-13, Bonn;

GoffmanN, E. (1972): Asyle, über die soziale Situation psychiatrischer Patienten und anderer Insassen, Frankfurt am Main;

GÖRTEMAKER, M. (2002): Kleine Geschichte der Bundesrepublik Deutschland, München;

GRÄF, L. (1993): Privatheit und Datenschutz, eine soziologische Analyse aktueller Regelungen zum Schutz privater Bereiche auf dem Hintergrund einer Soziologie der Privatheit, Köln 
Grewe, W. (1985): Der Übergang vom Besatzungsregime zu den Verträgen von Bonn und Paris, IN: Göttinger Arbeitskreis (Hrsg.): Deutschlandvertrag, westliches Bündnis und Wiedervereinigung, S. 7- 23, Berlin;

GREVE, W. (1988): Die Jahre der Entscheidung - Von der Besatzungsherrschaft zur Souveränität der Bundesrepublik Deutschland, IN: FUNKE, M. (1988): Entscheidungen für den Westen, Vom Besatzungsstatut zur Souveränität der Bundesrepublik 1949-1955, S. 93-115, Bonn;

GüNTHER, E. (1984): Lehrbuch der Genetik, Stuttgart;

HABERMAS, J. (1976): Strukturwandel der Öffentlichkeit, Untersuchungen zu einer Kategorie der bürgerlichen Gesellschaft, Neuwied, Berlin;

Hansen, M,/Krause C. (2003): Selbstdatenschutz, Sicherheit im Eigenbau, IN: HadDoutI, C. (Hrsg.): Bürgerrechte im Netz, S. 127-162, Bonn;

HAssemer, W. (2000): Die Wonne des Dabeiseins, Endgültig Teil der Familie: Big Brother, IN: SZ, Montag, 28. Februar 2000, S.17;

HASSEMER, W. (2002): Zum Spannungsverhältnis von Freiheit und Sicherheit, Drei Theses, IN: Vorgänge, Zeitschrift für Bürgerrechte und Gesellschaftspolitik Nr 159, September 2002, Freiheitsrechte in Zeiten des Terrors, S. 1-5, Opladen;

HAUCK, M. (2008): Ich bin schon drin, www.sueddeutsche.de/computer/artikel/818/160381/,27.02. 2008, 11.10 Uhr;

Hegel, G (1981): Grundlinie der Philosophie des Rechts oder Naturrecht und Staatswissen im Grundriss, nach der Ausgabe von Eduard Gans herausgegeben und mit einem Anhang versehen von Hermann Klenner, Berlin;

HEINE, P. (2004): Terror in Allahs Namen, Freiburg im Breisgau;

Hentschel, M./Pötzl, N. (1986): Die Position der RAF hat sich verbessert, der ehemalige BKA-Chef HORST HEROLD über Terroristen und Computerfahndung, DER SPIEGEL 37/1986 vom 08. 09.1986, Seite 38-61;

HeRnKIND, M. (2000): Personenkontrollen und Schleierfahndung, IN: Kritische Justiz, 33 (2000), S. 188-208; 
Herold, H. (1985): »Rasterfahndung« - eine computergestützte Fahndungsform der Polizei, IN: Recht und Politik, 21 (1985), S. 84-97, Berlin;

Hesse, J./Ellwein, T. (1997): Das Regierungssystem der Bundesrepublik Deutschland, Band 1: Text, Opladen, Wiesbaden;

Hesselberger, D. (1999): Das Grundgesetz, Kommentar für die politische Bildung, Neuwied; HIPPLER, J. (2004): Die Folgen des 11. September 2001 für die internationalen Beziehungen, IN: Aus Politik und Zeitgeschichte, B 3-4/2004, S. 3-7;

Hobbes, T (1970): Leviathan, erster und zweiter Teil, Stuttgart;

HocHSCHILD, U: (2005): Gewaltenteilung im Deutschen Bewusstsein, Versuch einer Kritik, http://gewaltenteilung.de/einf_druck.htm\#4, 20.12.2008, 11Uhr;

Hoffmann, H./Schoeller, W. (Hrsg.) (2001): Wendepunkt 11. September 2001, Terror, Islam und Demokratie, Köln;

Holzberger, M. (2000): §129b StGB Steilvorlage aus Europa, Mit EU-Druck zur Ausweitung des politischen Strafrechts,

http://www.freilassung.de/div/texte/129a/cilip66.htm, 10.12.2008, $10 \mathrm{Uhr}$;

Holzhaider, H. (2007): Prozess um den Mord in JVA Siegburg, www.sueddeutsche.de/panorama/artikel/420/126226/article.html, 3. 8. 2007, 17.52 Uhr;

Horwitz, A. (1990): The Logic of Social Control, New York;

HRADIL, S. (1999): Soziale Ungleichheit in Deutschland, Opladen;

Huthmann, K./Schweitzer, A. (2001): Alles im Griff-auch allein: Finanzielle Sicherheit für Alleinerziehende, Landsberg am Lech;

ID-Verlag (Hrsg.) (1997): Rote Armee Fraktion, Texte und Materialien zur Geschichte der RAF, Berlin;

Insitut für Demoskopie Allensbach (IdF) (2003): Der Wert der Freiheit, Ergebnisse einer Grundlagenstudie zum Freiheitsverständnis der Deutschen, Allensbach; 
IPSEN, J. (2003): Parteien, IN: SACHS, M. (Hrsg.): Grundgesetz, Kommentar, 890-940, München;

ISENSEE, J. (1983): Das Grundrecht auf Sicherheit, zu den Schutzpflichten des freiheitlichen Verfassungsstaates, Berlin, New York;

ISMAYR, W. (2001): Der Deutsche Bundestag im politischen System der Bundesrepublik Deutschland, Opladen;

JANISCH, W. (2002): »Terroristenparagraf«, Rechtsstaat in Zeiten des Terrors, http://www.stern.de/politik/historie/316838.html?eid=501091, 11.12.2008, 10.00 Uhr;

JONAS, H. (1972): Organismus und Freiheit, Ansätze einer philosophischen Biologie, Göttingen;

JÖRGES, H.-U. (2007): Der rasende Staat, IN: Stern, Nr 23, 2007, S.17;

Jungholt, T./Müller, P. (2008): Sicherheit, Schäuble greift Verfassungsrichter scharf an, http://www.welt.de/politik/article1571640/Schaeuble_greift_Verfassungsrichter_scharf_an.ht $\mathrm{ml}, 20.01 .2008,6.23 \mathrm{Uhr}$;

KANT, I. (2000): Kritik der praktischen Vernunft, Sämtliche Werke, Bd. 1, o. O.;

KASTILIAN, S. (2008): Molekulare Spuren kindlicher Gewalterfahrungen? http://www.faz.net/s/Rub268AB64801534CF288DF93BB89F2D797/Doc EFFAD72145EAD47 2498043FB4B095E97E ATpl Ecommon Scontent.html, 15.5.2008, 11.10 Uhr;

Kaufmann, F.-X. (1970): Sicherheit als soziologisches und sozialpolitisches Problem, Untersuchungen zu einer Wertidee hochdifferenzierter Gesellschaften, Stuttgart;

KeRSCHER, H. (2008): Super-GAU des Datenschutzes, http://www.sueddeutsche.de/computer/899/451610/text/, 15.12.15.42 Uhr;

KIER, F. (2003): Stapelweise Modrow, Gysi, Kant und Genossen, IN: JesSE, E. (Hrsg.): Eine Revolution und ihre Folgen, 14 Bürgerrechtler ziehen Bilanz, S.160-173, Berlin;

KıNZIG, J. (2004): Die rechtliche Bewältigung von Erscheinungsformen organisierter Kriminalität, Berlin; 
KLEIN, N . (2007): The Shock Doctrine, London, New York;

KNIESCHKE, W. (1926): Kismet oder Vorsehung, Berlin;

KNOBLOCH, H. (2005): Wissenssoziologie, Konstanz;

KORTE, K.-R. (2003): Wahlen in der Bundesrepublik Deutschland

Kovacıc, A. (1987): Der legale Terror, das Jahrzehnt der Scharfmacher, Bornheim-Merten;

KoYDL, W. (2008): Datenschutz in Großbritannien, englische Sammelwut, www.sueddetusche.de/politik/856/450577/text/, 6.12.2008, 11.59Uhr;

KREMPL, S: (2007): Google protestiert gegen deutsche Pläne zur Vorratsdatenspeicherung, http://www.heise.de/newsticker/Google-protestiert-gegen-deutsche-Plaene-zur-Vorratsdatenspeicherung--/meldung/85568, 20.02.2007, 11.30 Uhr;

KREMPL, S. (2008): Verfassungsschutz beharrt auf heimlichen Online-Durchsuchungen, http://www.heise.de/newsticker/Verfassungsschutz-beharrt-auf-heimlichen-Online-Durchsuchungen--/meldung/120110, 12.12.2008, $11 \mathrm{Uhr}$;

KREMPL, S./KUI, J. (2008): Der Schäuble-Katalog, von der Anti-Terrordatei zum Präventionsstaat,

http://www.heise.de/ct/07/09/038, 12.12.2008, $10 \mathrm{Uhr}$;

KRIELE, M. (1992): Einführung in die Staatslehre, die geschichtlichen Legitimitätsgrundlagen des demokratischen Verfassungsstaates, Opladen;

KRollman, H. (1966): Das Hessische Gesetz über die öffentliche Sicherheit und Ordnung, Stuttgart;

Krollmann, H. (1972): Das Hessische Gesetz über die öffentliche Sicherheit und Ordnung, Stuttgart;

KuHLmAnN, U. (2008): Reisepass mit OLED-Display

http://www.heise.de/newsticker/Reisepass-mit-OLED-Display--/meldung/108209/from/ atom10, 21.5.2008, $17 \mathrm{Uhr}$; 
KURI, J. (2008): Zahl der Kontenabfragen im Jahr 2007 auf fast 100.000 gestiegen, http://www.heise.de/newsticker/meldung/102720/from/atom10, 30.1. 2008, 13.54Uhr;

LACY, S. (2008): The Stories Of Facebook, Youtube \& Myspace, the people, the hype and the deals behind the giants of Web 2.0, Richmond;

LAMneK, S. (1979): Theorien abweichenden Verhaltens: eine Einführung für Soziologen, Psychologen, Pädagogen, Juristen, Politologen, Kommunikationswissenschaftler und Sozialarbeiter, München;

LAMNEK, S. (2003): Die Ambivalenz von Öffentlichkeit und Privatheit, IN: LAMNEK, S./TINNEFELD, M.-T. (Hrsg.): Privatheit, Garten und politische Kultur, von kommunikativen Zwischenräumen, S.40-66, Opladen;

LANGE, H.-J. (1999): Innere Sicherheit im politischen System der Bundesrepublik Deutschland, Wiesbaden;

LANGE, H.-J. (2006): Innere Sicherheit, IN: Lange, H.-J. (Hrsg.): Wörterbuch zur Inneren Slcherheit, S. 123-134, Wiesbaden;

LASKI, H. (1915): Die Souveränität des Staates, London;

LASKI, H. (1945): An Introduction to Politics, London;

LAutner, G. (1982): Die freiheitliche demokratische Grundordnung, Versuch einer Inhaltsklärung ihrer vom Bundesverfassungsgericht aufgeführten Elemente, Königstein;

LEGNARO, Aldo (1997): Konturen der Sicherheitsgesellschaft: Eine polemisch-futurologische Skizze, IN: Leviathan, Zeitschrift für Sozialwissenschaft, Jahrgang 1997, Bd, 2, S. 271-285, Opladen;

LEIDHOLD, W. (1987): Neue Konzepte zur europäischen Sicherheitspolitik und die asiatischpazifische Hemisphäre: Zusammenhänge, Probleme und Szenarien, IN: PAHR, W./RITTberger, V./Werbik, H: (Hrsg.): Europäische Sicherheit, Prinzipien, Perspektiven, Konzepte, Wien;

Leutheusser-Schnarrenberger, S: (2005): Gefährdet der Terrorismus den liberalen Verfassungsstaat? IN: KNOP, K./NEISSER, H. (Hrsg.): Countering Modern Terrorism - History, 
Current Issues and Future Threats, 2. Internationale Sicherheitskonferenz, S. 273-287, Bielefeld;

LEYENDECKER, H. (2007): Sie haben sich geirrt, Mr ORWELL, IN: SZ, 10.01. 2007, 2;

LEYENDECKER, H. (2008): BND-Bespitzelungsaffäre, Sie wussten, was sie taten, www.sueddeutsche.de/deutschland/artikel/676/171175/, 24.04.2008, 17.04 Uhr;

Lindenberg, M./SCHMIDT-SemisCH, H. (1995): Sanktionsverzicht statt Herrschaftsverlust: Vom Übergang in die Kontrollgesellschaft, IN: KrimJ, 27. Jhg., Heft 1, S. 2-18, Weinheim;

LOCKE, J. (1974): Über die Regierung, Stuttgart;

Lohmann, D. (2002): Rekordjagden in der Arktis,

http://www.scinexx.de/dossier-detail-59-18.html, 10.9.2008, 17.19 Uhr;

Löw, K. (1997): Der Staat des Grundgesetzes, München;

Lummann, N. (1995): Die Realität der Massenmedien, Opladen;

LUthE, H. O. (1985): Distanz, Untersuchung einer vernachlässigten Kategorie, München;

LYon, D. (1994): The Electronic Eye, The Rise of Surveillance Society, Minneapolis;

Lyon, D. (2006): The search for surveillance theories, IN: Lyon, D. (Hrsg.): Theorizing Surveillance, the panopticon and beyond, S. 3-21, Portland;

LYON, D. (2009): Identifying Citizens, ID Cards as Surveillance, Cambridge (UK), Malden (USA);

MAAEN, S. (1999): Wissenssoziologie, Bielefeld;

Mahlmann, P./Schindelhauer, C. (2007): Peer-to-Peer-Netzwerke, Algorithmen und Methoden, Berlin;

MARCUSE, L. (1981): Das Märchen von der Sicherheit, Zürich;

Markowitsch, H. (2006): Gene, Meme, »freier Wille«: Persönlichkeit als Produkt von Nervensystem und Umwelt, IN: ReichertZ, J./ZABoura, N. (Hrsg.): Akteur Gehirn - oder das vermeintliche Ende des handelnden Subjekts, eine Kontroverse, S. 31-47 Wiesbaden; 
MAYNTZ, R. (2006): Hierarchie oder Netzwerk? Zu den Organisationsformen des Terrorismus, In: Berliner Journal für Soziologie, Band 14, Nr. 2, S. 251-262;

Mayntz, R./Holm, K./Hübner, P. (1972): Einführung in die Methoden der empirischen Soziologie, Opladen;

McKenzie, E. (1994): Privatopia, Homeownder Associations and the Rise of Residential Private Government, New Haven, London;

Merton, R. (1965): Die Eigendynamik gesellschaftlicher Voraussagen, IN: ToPITSCH, E. (Hrsg.): Lodik der Sozialwissenschafter, S. 144-165, Köln, Berlin;

Merton, R. (1995): The Thomas Theorem and The Matthew Effect, IN: Social Forces, Dezember 1995, S. 379-424, Chapel Hill (CA);

Merton, R. (1968): Social Theory and Social Structure, New York;

Meyer-Wieck, H. (2004): Rechtswirklichkeit und Effizienz der akustischen Wohnraumüberwachung ("großer Lauschangriff») nach $\S 100$ c Abs. 1 Nr. 3 StPO, Eine rechtstatsächliche Untersuchung im Auftrag des Bundesministeriums der Justiz,

http://www.bmj.bund.de/media/archive/786.pdf, 18.12.2008, 11 Uhr;

Mıкоs, L. u.a. (2000): Im Auge der Kamera, das Fernsehereignis Big Brother, Berlin;

Montesquieu, CH. (1992): Vom Geist der Gesetze, Tübingen;

Muehlbauer, P. (2008): BND soll »Online-Durchsuchungen« bei Mitarbeitern durchgeführt haben,

http://www.heise.de/newsticker/BND-soll-Online-Durchsuchungen-bei-Mitarbeitern-durchgefuehrt-haben--/meldung/120063, 8.12.2008, 8.52 Uhr;

MüLler, A. (2007): Sterben, Tod und Unsterblichkeit im Sport, eine existenzphilosophische Deutung, Berlin;

Murck, M. (1980): Soziologie der öffentlichen Sicherheit: Eine staatliche Aufgabe aus Sicht der Bürger, Frankfurt am Main, New York; 
NASE, H. (2003): Die Europäische Union im Alltag der Bürger, IN: NASE, H. u.a. (Hrsg.): Das Europa der Bürgerlnnen, die Europäische Union im Alltag, S.14-79, Hamburg;

NeidhaRdt, F: (1982): Soziale Bedingungen terroristischen Handelns, Das Beispiel der "Baader-Meinhof-Gruppe« (RAF), IN: Baeyer-Katte, W. von et. al. (Hrsg): Gruppenprozesse, Analysen zum Terrorismus 3, S. 317-391, Opladen;

NeIDHARdt, F. (2006): Akteure und Interaktionen, Zur Soziologie des Terrorismus, IN: KRAUSHAAR, W. (Hrsg.): Die RAF und der linke Terrorismus, Bd. 1, S. 123-137, Hamburg;

Neusel, H. (1996): Das Verhältnis privater Sicherheitsdienste zur Polizei, IN: HIRSCHER, G. (Hrsg): Zur Lage der inneren Sicherheit in Deutschland, S. 82-102, München;

Nielsen, J./Tsuboi, T./Sturup, G./Romano, D. (1968): XYY chromosomal constitution in criminal psychopaths, Lancet, Vol. 7, S.576, 0. 0;

Nuscheler, F./Steffani, W. (Hrsg.): Pluralismus, Konzeptionen und Kontroversen, München;

OberReuter, H. (1978): Notstand und Demokratie, vom monarchischen Obrigkeits- zum demokratischen Rechtsstaat, München;

OHLER, N. (1986): Reisen im Mittelalter, München;

Parsons, T. (1966): Der Begriff der Gesellschaft, IN: PARsons , T. (Hrsg.): Zur Theorie sozialer Systeme, herausgegeben und eingeleitet von Stefan Jensen, 121-156, Opladen;

Parsons, T. (1970): The social System, London;

Patalong, F. (2008): Selbstmord im Internet, http://www.spiegel.de/netzwelt/web/o,1518,591783,00.html, 21.11. 2008, 8 Uhr;

Pauen, M. (2004): Illusion Freiheit? Mögliche und unmögliche Konsequenzen der Hirnforschung. S.Fischer, Frankfurt am Main;

Peters, H. (1989): Devianz und soziale Kontrolle, eine Einführung in die Soziologie abweichenden Verhaltens, Weinheim, München;

POPPER, K. (1934): Logik der Forschung, Tübingen; 
Pöтzsch, H. (2001): Die deutsche Demokratie, Bonn;

PrantL, H. (2002): Verdächtig, Hamburg, Wien;

PrantL, H. (2004): Völlerei und Polizei, verdachts- und ereignisunabhängig: die Schleierfahndung als Inbegriff der Sicherheitspolitik, SZ, 10.11.2004, S. 3;

PRANTL, H. (2007): Der artgerechte Staat, www.sueddeutsche.de/deutschland/artikel/888/117759/article.html, 27.6.2007, 10.53 Uhr;

Prantl, H. (2008a): BKA-Gesetz, Auf Biegen und Brechen, www. sueddeutsche.de/politik/485/450207/text/, 12.12.2008, 11Uhr;

PrantL, H. (2008 b): Die Daten und ihr Schutz, Unternehmen behandeln die Daten der Bürger, als handele es sich um Altpapier. Die Sensibilität für den Datenschutz ist verloren gegangen, Dabei bietet nur er Sicherheit in der digitalen Welt,

www.sueddeutsche.de/politik/793/451505/text/, 15.12.2008, 1 Uhr;

Pratchett, T./Stewart, I./Cohen, J. (1999): The Science of Discworld, London;

Pratchett, T. (2004): Going postal, London;

PRECHTL, P. (1995): Hume, David, IN: Metzler Philosophenlexikon, Von den Vorsokratikern bis zu den Neuen Philosophen, S.408-411, Stutgart, Weimar;

REDELFS, M. (2003): Informationsfreiheitsrechte in Deutschland, Der langsame Abschied vom »Amtsgeheimnis«, IN: SCHULZKI-HAddoutI, C. (Hrsg.): Bürgerrechte im Netz, S.190-210, Bonn;

Regan, P. (1996): Genetic Testing and Workshop Surveillance: Implications for Privacy, IN: Lyon, D./Zureik, E. (Hrsg.): Computers, Surveillance and Privacy, S. 21-47, Minneapolis, London;

REISCHL, G. (2008): Die Google Falle, die unkontrollierte Weltmacht im Internet, Wien;

REUTER, K. (1990): Föderalismus, Grundlagen und Wirkungen in der Bundesrepublik Deutschland, Heidelberg;

Risse-Kappen, T. (1988): Die Krise der Sicherheitspolitik: Neuorientierungen und Ent- 
scheidungsprozesse im politischen System der Bundesrepublik Deutschland 1977-1984, Mainz, München;

RöD, W. (1964): Descartes, München;

RöscH, G. (2005): Einleitung, IN: RöscH, G. (Hrsg.): Codes, Geheimtext und Verschlüsselung, Geschichte und Gegenwart einer Kulturpraxis, S.7-23, Tübingen;

Rössler, B. (2001): Der Wert des Privaten, Frankfurt am Main;

Rötzer, F. (2007): Die Mutter aller Terror-Datenbanken quillt über, http://www.heise.de/tp/r4/artikel/24/24939/1.html, 26.3.2007, 11 Uhr;

Rousseau, J.-J. (1964): Euvres complètes, III., Du contrat social, Écrits politiques, Paris;

Rousseau, J.-J. (1986): Vom Gesellschaftsvertrag oder Grundsätze des Staatsrechts, Stuttgart;

RudzıO, W. (2000): Das politische System der Bundesrepublik Deutschland, Opladen;

SACHS, M. (2007): Verfassungsgrundsätze; Widerstandsrecht, IN: SACHS, M. (Hrsg.): Grundgesetz, Kommentar, S. 766-824, München;

SÄCKER, H. (1995): Gesetzgebung durch das BVerfG - Das BVerfG und die Legislative - IN: PIAzolo, M. (Hrsg.): Das Bundesverfassungsgericht, Ein Gericht im Schnittpunkt von Recht und Politik, S. 189-227, Mainz, München;

SCHAAR, P. (2007): Tätigkeitsbericht 2005-2006, 21. Tätigkeitsbericht, Berlin;

SCHÄFER, F. (1966): Die Notstandsgesetze, Vorsorge für den Menschen und den demokratischen Rechtsstaat, Opladen;

SCHÄFER, U. (2008): Spitzel-Affäre, Telekom verspielt das Vertrauen der Bürger, www.sueddeutsche.de/wirtschaft/artikel/272/176737/, 26.05.2008, 7.35 Uhr;

SCHAMBECK, H. (1999): Grundprobleme des parlamentarischen Regierungssystems, IN: ISENSEe, J./LeCheleR, H. (Hrsg.): Freiheit und Eigentum, Festschrift für Walter Leisner zum 70. Geburtstag, S. 449-466, Berlin; 
SCHEerer, S. (1980): Gesetzgebung im Belagerungszustand, IN: Blankenburg, E. (Hrsg.): Politik der Inneren Sicherheit, S.120-169, Frankfurt am Main;

SCHeerer, S. (2000): "Soziale Kontrolle« - schöner Begriff für böse Dinge?, IN: Peters, H. (Hrsg.): Soziale Kontrolle, zum Problem der Nonkonformität in der Gesellschaft, S.154-169, Opladen;

SCHMID, T. (2008): Warum ein bisschen Überwachung nicht reicht, http://www.welt.de/politik/article2730526/Warum-ein-bisschen-Ueberwachung-nichtreicht.html, 15.11.2008, 17.02 Uhr;

SCHmidt, J. (1998): Die Grenzen der Rational Choice Theorie, Opladen;

SCHMIDT, M. (2000): Demokratietheorien, München;

SCHMIDT-PREUSS, M. (1999): Gestaltungskräfte im parlamentarischen Regierungssystem der Bundesrepublik Deutschland, IN: ISEnSEe, J./LeCheler, H. (Hrsg.): Freiheit und Eigentum, Festschrift für Walter Leisner zum 70. Geburtstag, S. 467-484, Berlin;

SCHMieder, J./HAuck, M. (2008): Trojaner aus China, Deutsche Tölpelei, http://www.sueddeutsche.de/computer/614/416381/text/, 17.08. 2007, 13.55 Uhr;

SCHNEIDER, C. (2006): Willkürliche Kontrollen sind unzulässig, IN: SZ, 10. 2. 2006, S.40;

SCHNEIER, B. (2001): Secrets \& Lies, IT-Sicherheit in einer vernetzten Welt, Heidelberg, Weinheim;

SChnepper, M. (2004): Robert K. Mertons Theorie der self-fulfilling prophecy, Adaption eines soziologischen Klassiker, Frankfurt am Main;

SCHOller, H./Bross, S. (1978): Grundzüge des Polizei- und Ordnungsrechts in der Bundesrepublik Deutschland, Heidelberg, Karlsruhe;

Schulze, G. (2000): Erlebnisgesellschaft, IN: Aus Politik und Zeitgeschichte, 17.3.2000, B 12/2000, Bonn;

Schulzki-Haddouti, C./Redelfs, M. (2003): Informationsfreiheit als demokratisches Prinzip, Mehr Transparenz durch mehr Information, IN: SchulzkI-HAddoutI, C. (Hrsg.): Bürgerrechte im Netz, S.178-19o, Bonn; 
SCHÜLER-SPRINGORUM, H. (1991): Kriminalpolitik für Menschen, Frankfurt am Main;

SCHÜLER-SPRINGORUM, H. (1996): Wider den Sachzwang, München;

Schütz, A. (1977): Parsons Theorie des sozialen Handelns, IN: Sprondel, M. (Hrsg.): Alfred Schütz, Talcott Parsons, zur Theorie sozialen Handelns, Frankfurt am Main;

SCHWARTZ, R. (2008): Rumänien: Senat verordnet positive Nachrichten, http://www.dw-world.de/dw/article/o,2144,3442277,00.html, 12. 8. 2008, 18 Uhr;

SCHWENK, J. (2002): Sicherheit und Kryptographie im Internet, Braunschweig, Wiesbaden;

SEXL, R./SCHMIDT H. K. (2000): Raum-Zeit-Relativität, Wiesbaden;

SIETMANN, R. (2007): Innere Sicherheit: Auf der Rutschbahn von Ausnahmegesetzen, http://www.heise.de/newsticker/meldung/91183/from/atom10, 12.12. 2008, 20.34 Uhr;

Simmel, G. (1968): Soziologie, Untersuchungen über die Formen der Vorgesellschaftung, Berlin;

Singelnstein, T./Stolle, P. (2006): Die Sicherheitsgesellschaft, soziale Kontrolle im 21. Jahrhundert, Wisebaden;

SINGER, W. (2007): Der Beobachter im Gehirn: Essays zur Hirnforschung, Frankfurt am Main;

SINGH, S. (2001): Codes, München, Wien;

SÖHLER, M. (2008): Die vielen Daten des Herrn TeSCHKE, http://www.netzeitung.de/internet/1129497.html, 20.08.2008, $11 \mathrm{Uhr}$;

Sontheimer, K. (1957): Pluralismus, IN: Fraenkel, E./Bracher, K. (Hrsg.): Staat und Politik, S. 254-257, Frankfurt am Main;

Sontheimer, K./Bleek, W. (2000): Grundzüge des politischen Systems der Bundesrepublik Deutschland, Bonn;

SPÖRL, K.-H. (1997): Zur Einführung einer verdachts- und ereignisunabhängigen Personenkontrolle (»Schleierfahndung«) in Bayern, Die Polizei, 88.Jahrgang, Heft 8, August 1997, S. 217-244; 
Steffan I, W. (1977): Pluralismus, Konzeptionen - Positionen - Kritik, IN: GageL, W. (Hrsg.): Pluralismus, Politische Bildung, Beiträge zur wissenschaftlichen Grundlegung und zur Unterrichtspraxis, S. 3-33, Stuttgart;

Steinberger, P. (2007): Überbehütete Kinder, Big Mother is watching you, www. sueddeutsche.de/leben/artikel/757/118619/article.html, 18. 06.2007, 15.05 Uhr;

Stern, K. (1984): Das Staatsrecht der Bundesrepublik Deutschland, München;

Sternberger, D. (1982): Verfassungspatriotismus, Grundfragen der Demokratie, Hannover;

Strasser, H./Schweer, T. (2008): Ausblick: Auf dem Weg in die Präventionsgesellschaft?, IN: Strasser, H./Zdun, S. (Hrsg.): Das da draußen ist ein Zoo und wir sind die Dompteure, S. 165-182, Wiesbaden;

Strasser, P. (2005): Das neue Kontrolldenken in der Kriminologie, IN: Krim. Journal, 37. Jhg., H. 1, S. 39-52, Weinheim;

StRöbele, H.-CH. (1998): Zur Geschichte der RAF,

http://www.stroebele-online.de/dokumente/1021.html, 10.12.2008, $10 \mathrm{Uhr}$;

StRöм, P. (2003): Die Überwachungsmafia, das gute Geschäft mit unseren Daten, München, Wien;

StURM, M. (2003): Deutschland nach der Einheit - mehr als eine erweiterte Bundesrepublik, IN: WANKE, G. (Hrsg.): Über die Folgen der Einheit, S.115-132, Erlangen;

Sturm, M. (2006): Tupamaros München: »Bewaffneter Kampf«, Subkultur und Polizei 1969-1970, IN: WeINHAUER, K. u.a. (Hrsg.): Terrorismus in der Bundesrepublik, Medien, Staat und Subkulturen in den 1970 er Jahren, S. 99-134, Frankfurt, New York;

SutoR, B. (1994): Politik, ein Studienbuch zur politischen Bildung, Paderborn;

ThIEL, E. (1989): Die Europäische Gemeinschaft, vom Gemeinsamen Markt zur Europäischen Union, München;

Thomas, W./Thomas, D. (1928): The Child in America, New York; 
Tinnefeld, M.-Th./EhmanN, E./Gerling, W. (2005): Einführung in das Datenschutzrecht, Datenschutz und Informationsfreiheit in europäischer Sicht, München, Wien;

Tipler, P./ Llewellyn, R. (2003): Moderne Physik, München;

UEBERSCHAER, H.-C. (1956): Bundesverfassungsgericht - Rechtsstaat - Justizstaat, ein Beitrag zur Frage einer Suprematie des Bundesverfassungsgerichtes in der rechtsstaatlichen Ordnung des Grundgesetzes, Heidelberg;

UNO (1948): Die Allgemeine Erklärung der Menschenrechte, Resolution 217 A (III), New York;

VEIT, O. (1957): Soziologie der Freiheit, Frankfurt am Main;

Waldmann, E. (1968): Notstand und Demokratie, Studie zur Situation in der Bundesrepublik Deutschland, Boppard;

WaLdmAnN, P. (2005): Terrorismus, Provokation der Macht, Hamburg;

WALLACE, A. (1871): Contributions to the theory of natural selection. A series of essays, New York;

Watzlawick, P. (1983): Anleitung zum Unglücklichsein, München, Zürich

Weber, J. (1981): Regieren unter alliierter Aufsicht, Besatzungsstatut und Petersberger Abkommen; IN: Weber, J. (Hrsg.): Die Gründung des neuen Staates 1949, S. 198-214, München;

Weber, M. (1921): Soziologische Grundbegriffe, IN: Winckelmann, J. (Hrsg., 1988): MaX WebER, Gesammelte Aufsätze zur Wissenschaftslehre, S. 541-581, Tübingen;

Weber, M. (1976): Wirtschaft und Gesellschaft, Studienausgabe, Tübingen;

Wehrheim, J. (2008): Gated Communities, Sicherheit und Separation in den USA , http://www.citycrimecontrol.net/texte/gated.html\#f14, 12.12.2008, $11 \mathrm{Uhr}$;

WeICHERT, T. (2004): Datenschutz der Spaß macht, IN: BIZER u.a. (Hrsg.): Innovativer Datenschutz 1992-2004, Wünsche, Wege, Wirklichkeit, Für Helmut Bäumler, S. 129-147, Kiel;

WEIDENFELD (2002): Europäische Einigung im historischen Überblick, IN: WeIDENFELD, 
W./Wessels, W. (Hrsg.): Europa von A bis Z, Taschenbuch der europäischen Integration, S. 10-51, Bonn;

Weigel T./SChelL, M. (1994): Tage, die Deutschland und die Welt veränderten, München;

Weinhauer, K./Requate, J. (2006): Einleitung: Die Herausforderung des »Linksterrorismus", IN: Weinhauer, K. U.a. (Hrsg.): Terrorismus in der Bundesrepublik, Medien, Staat und Subkulturen in den 1970 er Jahren, S. 9-33, Frankfurt, New York;

WEISS, K. (2001): Das Ende der DDR, Runder Tisch und Demokratiebewegung, IN: Bayerische Landeszentrale für politische Bildungsarbeit (Hrsg.): Die zweite gesamtdeutsche Demokratie, S. 218-227, München

Westin, A. (1967): Privacy and Freedom, London, Sydney, Toronto;

Westin, A./Baker, M. (1972): Databanks in a Free Society, Computers, Record-Keeping and Privacy, New York;

WIDMER, A. (1995): Sicherheit - über Begrifflichkeit und staatliche Maßnahmen, IN: Bauhofer S./Bolle, P.-H. (Hrsg.): Innere Sicherheit - Innere Unsicherheit? Reihe Kriminologie, Bd.13, Zürich;

Wiedemann, H.-R./ Kunze, J./Spranger, J. (2001): Atlas der klinischen Syndrome, für Klinik und Praxis, Stuttgart, New York;

WILKENS, A. (2008): Google wirft EU-Datenschützern den Ball zurück, http://www.heise.de/newsticker/meldung/91023/from/atom10 12.06.2007, 15.07 Uhr;

Wohlfahrt, N. (2005): Was opfern wir dem Markt? Hintergründe und Folgen der Privatisierung staatlicher Aufgaben, IN: KEICHER, R./AnHoRn, R. (Hrsg.): Privatisierung als Chance? Straffälligenhilfe zwischen marktwirtschaftlicher und staatlicher Steuerung, S.45-59, Freiburg;

Wohlfeld, M. (1998): Sicherheit, IN: Bertelsmann Stiftung (Hrsg.): Kosten, Nutzen und Chancen der Osterweiterung für die Europäische Union, S.41-50, Gütersloh;

WOYKE, W: (1997): Die Europäische Union vor dem Eintritt in das 21.Jahrhundert, IN: 
WOYkE, W. (Hrsg.): Die Europäische Union, S. 9-25, Schwalbach;

Wuketits, M. (1981): Biologie und Kausalität, Biologische Ansätze zur Kausalität, Determination und Freiheit, Berlin und Hamburg;

ZImmermann, P (1999): Why I wrote PGP,

http://www.philzimmermann.com/EN/essays/WhylWrotePGP.html 
Ich erkläre an Eides Statt, dass ich die vorliegende Arbeit ohne unzulässige Hilfe Dritter und ohne Benutzung anderer als der angegebenen Schriften und Hilfsmittel angefertigt und die den benutzten Werken wörtlich oder inhaltlich entnommenen Stellen kenntlich gemacht habe.

Insbesondere habe ich für die Erstellung der Dissertation keine entgeltliche Hilfe von Vermittlungs- beziehungsweise Beratungsdiensten (Promotionsberatern oder anderen Personen) in Anspruch genommen.

Eichstätt, den 
Ich erkläre an Eides Statt, dass ich bereits früher keine Promotionsversuche unternommen oder abgeschlossen habe und die Dissertation in gleicher oder anderer Form in einem anderen Versuch oder in einem anderen Prüfungsverfahren vorgelegt habe.

Eichstätt, den

Ort, Datum

Unterschrift 
Ich erkläre an Eides Statt, dass ich an keiner Hochschule eine Doktorprüfung endgültig nicht bestanden habe ( $§ 5(5))$. 


\title{
Lebenslauf
}

\author{
Name Hornig \\ Vorname Jens Christian \\ Geburtstag 29. September 1976 \\ Nationalität deutsch \\ Stand ledig \\ Wohnort 85072 Eichstätt, Rosenweg 13 \\ Schulen September 1982 - Juli 1986 Grundschule Süd Moosburg \\ September 1986 - 30. Juli 1996 \\ Karl-Ritter-von-Frisch-Gymnasium Moosburg \\ Abitur 1996 \\ Universität Katholische Universität Eichstätt \\ Magisterstudiengang Politikwissenschaft, Soziologie, \\ Philosophie \\ von Wintersemester 1997/1998 - Juli 2003 \\ Wintersemester 1998/99 Urlaubssemester Bundeswehr \\ Aufbaustudiengang Erwachsenenbildung 2003/2004 \\ Promotionsstudiengang im Anschluss \\ Antrag auf Zulassung zum Promotionsverfahren Juni 2009 \\ Thema der Dissertation im Fach Soziologie: \\ Sicherheit statt Freiheit? \\ Betreuung der Doktorarbeit durch Herrn \\ Prof. Dr. Siegfried Lamnek (Hauptfachprüfer) \\ Prof. Dr. Jens Luedtke (Koreferent) \\ Prüfer in Politkwissenschaft (1. Nebenfach): Herr Prof. Dr. Detjen \\ Prüfer in Philosophie (2. Nebenfach): Herr. Prof. Dr. Fetz
}

